\title{
An Experimental Characterisation of the Velocities of the Spray Issued from a Pressurised Metered-Dose Inhaler
}

By

\author{
Brian Michael Crosland \\ Bachelor of Science in Aerospace Engineering, Diplôme d'Ingénieur
}

\author{
A thesis submitted to \\ The Faculty of Graduate Studies and Research \\ in partial fulfilment of \\ the requirements for the degree of \\ Master of Applied Science \\ Ottawa-Carleton Institute for \\ Mechanical and Aerospace Engineering
Department of Mechanical and Aerospace Engineering
Carleton University
Ottawa, Ontario \\ Canada
}

\author{
August 2008 (C) \\ 2008 - Brian Michael Crosland
}




$\begin{array}{ll}\begin{array}{l}\text { Library and } \\ \text { Archives Canada }\end{array} & \begin{array}{l}\text { Bibliothèque et } \\ \text { Archives Canada }\end{array} \\ \begin{array}{l}\text { Published Heritage } \\ \text { Branch }\end{array} & \begin{array}{l}\text { Direction du } \\ \text { Patrimoine de l'édition }\end{array} \\ \begin{array}{l}\text { 395 Wellington Street } \\ \text { Ottawa ON K1A ON4 } \\ \text { Canada }\end{array} & \begin{array}{l}\text { 395, rue Wellington } \\ \text { Ottawa ON K1A 0N4 } \\ \text { Canada }\end{array}\end{array}$

Your file Votre référence ISBN: 978-0-494-44035-3

Our file Notre référence

ISBN: 978-0-494-44035-3

NOTICE:

The author has granted a nonexclusive license allowing Library and Archives Canada to reproduce, publish, archive, preserve, conserve, communicate to the public by telecommunication or on the Internet, loan, distribute and sell theses worldwide, for commercial or noncommercial purposes, in microform, paper, electronic and/or any other formats.

The author retains copyright ownership and moral rights in this thesis. Neither the thesis nor substantial extracts from it may be printed or otherwise reproduced without the author's permission.
AVIS:

L'auteur a accordé une licence non exclusive permettant à la Bibliothèque et Archives Canada de reproduire, publier, archiver, sauvegarder, conserver, transmettre au public par télécommunication ou par l'Internet, prêter, distribuer et vendre des thèses partout dans le monde, à des fins commerciales ou autres, sur support microforme, papier, électronique et/ou autres formats.

L'auteur conserve la propriété du droit d'auteur et des droits moraux qui protège cette thèse. $\mathrm{Ni}$ la thèse ni des extraits substantiels de celle-ci ne doivent être imprimés ou autrement reproduits sans son autorisation.
In compliance with the Canadian

Privacy Act some supporting forms may have been removed from this thesis.

While these forms may be included in the document page count, their removal does not represent any loss of content from the thesis.
Conformément à la loi canadienne sur la protection de la vie privée, quelques formulaires secondaires ont été enlevés de cette thèse.

Bien que ces formulaires aient inclus dans la pagination, il n'y aura aucun contenu manquant.

\section{Canada}




\section{Abstract}

Pressurised metered dose inhalers (pMDIs) are widely used to deliver aerosolized medications to the lungs. Numerical simulations are often used to predict the flow and deposition of particles, requiring detailed information about the spray generated by a pMDI to ensure their validity. Presented here are detailed, spatially-resolved velocity measurements of the spray emitted from salbutamol sulfate pMDIs obtained using particle image velocimetry (PIV). Instantaneous planar velocity measurements were taken and ensemble-averaged at various times during the spray event. The mean spray velocities were shown to be bimodal in time with two velocity peaks. Planar velocity data were analyzed to produce prescriptive velocity profiles suitable for use in numerical simulations. Statistical comparisons from several thousand spray events indicate that there is no significant difference in measured velocity between i) two brands of pMDI canisters, ii) two similar pMDIs from different lots, and iii) a full pMDI versus an almostempty pMDI. Further experiments with a secondary air flow of 30 SLPM with and without an added cylindrical spacer deflected the spray downward, but had little effect on the velocity magnitude. 


\section{Acknowledgements}

Thanks,

To my mother, for all her support despite the three countries, two continents and two provinces my studies have led me to.

To my thesis supervisors, Professors Matthew Johnson and Edgar Matida, who provided just the right amount of direction and freedom to me on this project.

To Alex, Kevin, Steve, Stefan, Gary and Fred, for all their help designing, building, and setting up my experiments.

To Nancy, Marlene, and Christie, for having the answers to all my questions, or at least knowing where to find them.

To my friends, for giving up their office so that I could have more lab space.

Et enfin, à ma chère Catherine, je te remercie pour tout ton soutien, ton amour, et ton patience. 


\section{Table of Contents}

Abstract

Acknowledgements $\quad$ iv

Table of Contents $\quad$ v

List of Tables $\quad$ ix

List of Figures $\quad$ xi

Nomenclature $\quad$ xvii

Chapter 1: Introduction $\quad 1$

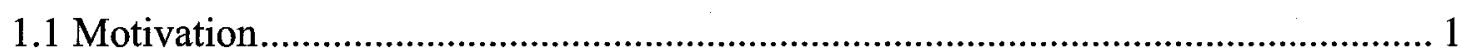

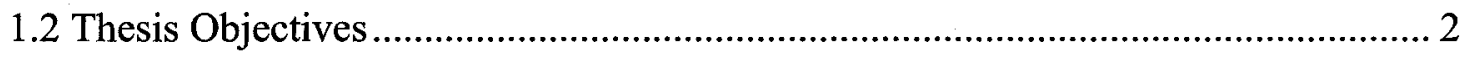

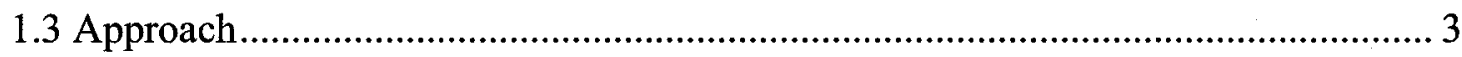

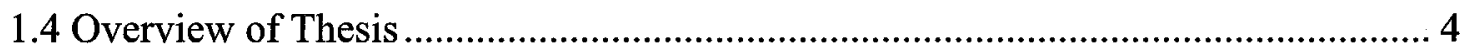

Chapter 2: Background and Literature Review 5

2.1 Introduction

2.2 Nebulizers and Dry Powder Inhalers .............................................................. 5

2.3 Pressurised Metered-Dose Inhalers........................................................................ 7

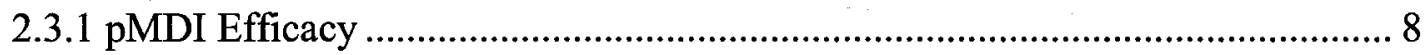

2.3.2 Characterisation of pMDI Spray Velocities................................................. 12

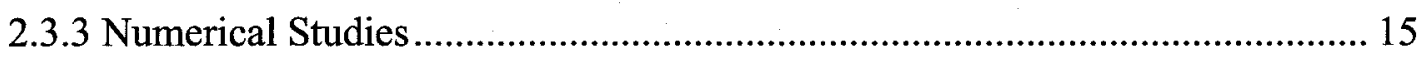

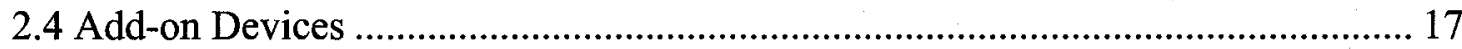

Chapter 3: Experimental Setup and Procedure 19 


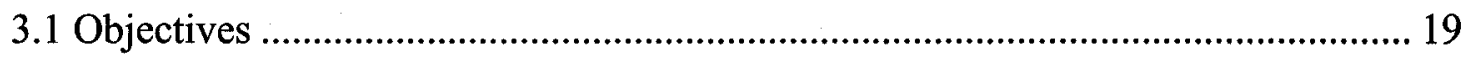

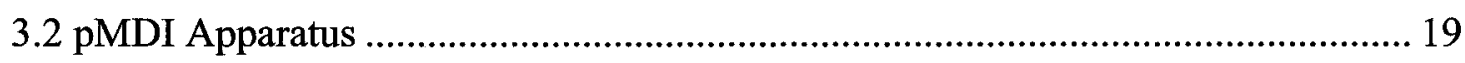

3.3 Air Flow Apparatus.................................................................................... 22

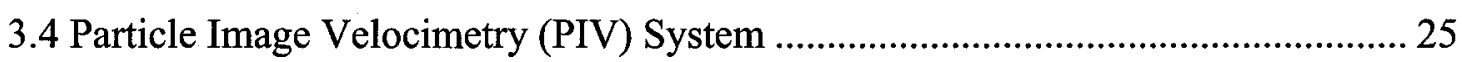

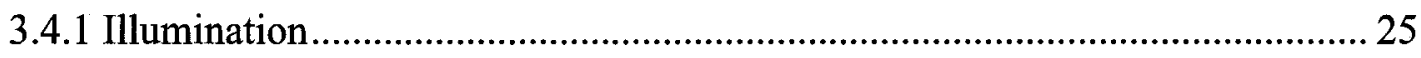

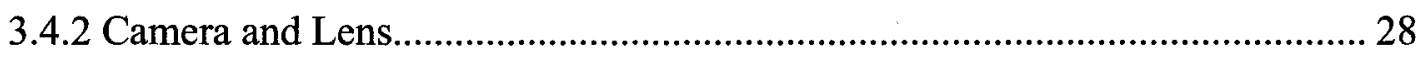

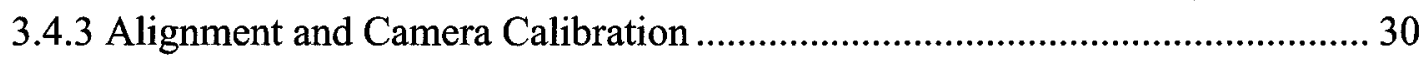

3.4.4 Triggering the PIV Data Acquisition Process................................................... 36

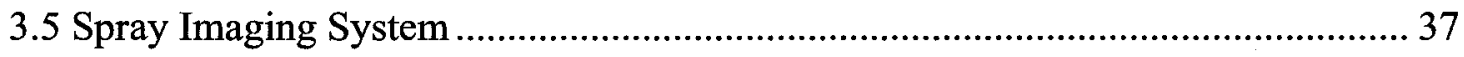

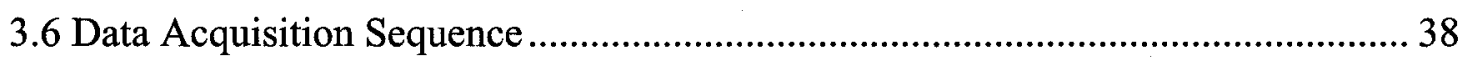

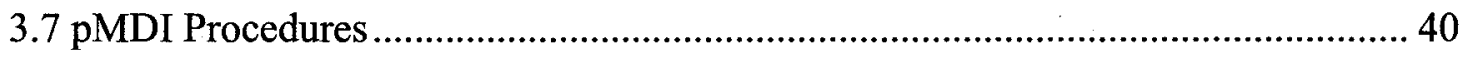

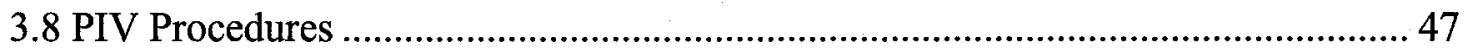

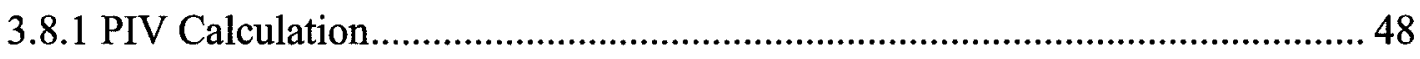

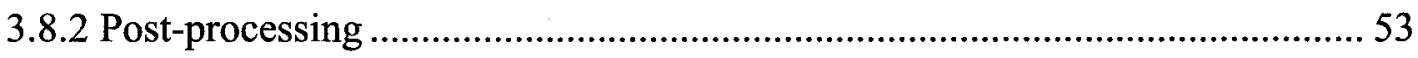

3.9 Spray Geometry Procedures ............................................................................... 58

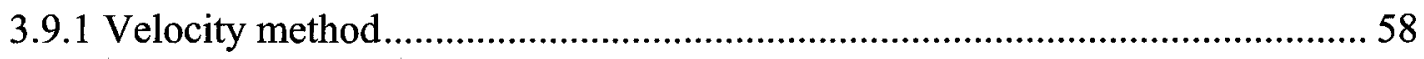

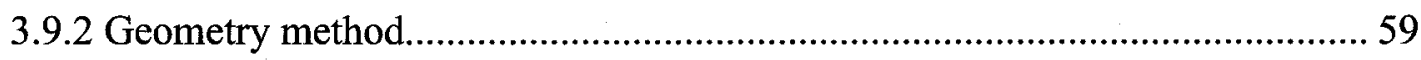

3.10 Add-On Device Apparatus and Procedures ........................................................... 62

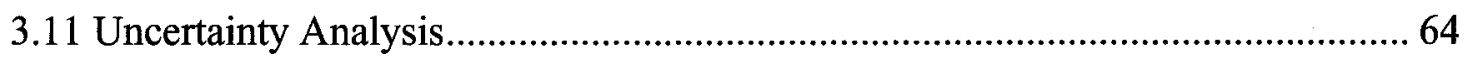

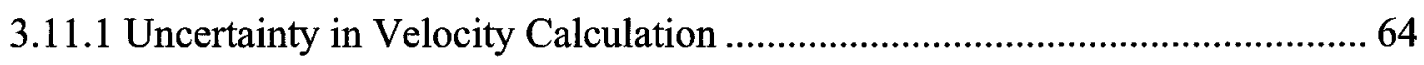

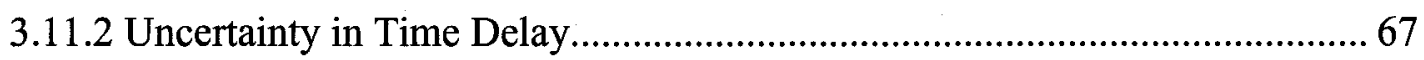

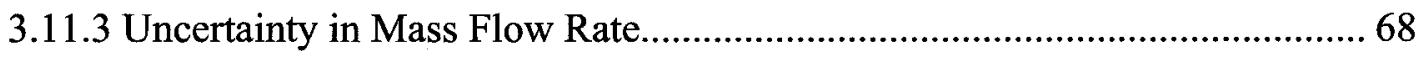

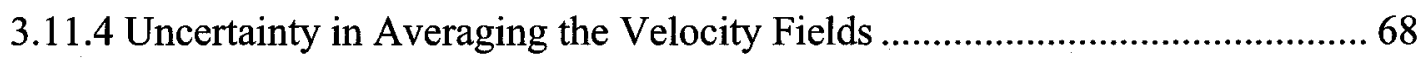

Chapter 4: Experimental Results $\quad 72$

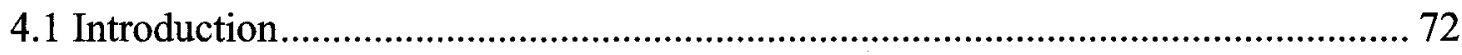

4.2 Spray Issued Into Open, Quiescent Air............................................................ 72

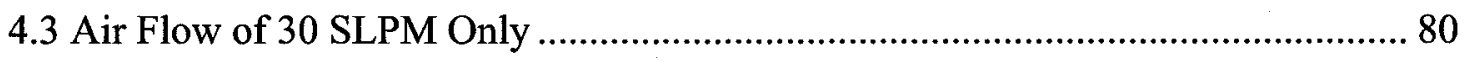

4.4 Spray Issued Into Open Air with 30 SLPM Air Flow ........................................... 83

4.5 Spray Issued Into Add-On Device with 30 SLPM Air Flow …………………….... 86

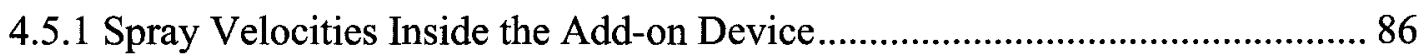


$\begin{array}{lr}\text { Chapter 5: Analysis and Discussion } & 91\end{array}$

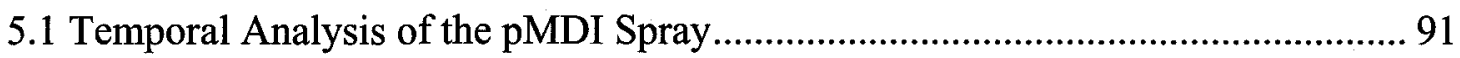

5.2 Analysis of Geometric Characteristics of pMDI Spray ………………………....... 93

5.3 Spray Issued by an Almost-Full versus an Almost-Empty Canister......................... 97

5.4 Spray Issued by a Ventolin- versus a ratiopharm-Brand Canister........................... 99

5.5 Average Turbulent Kinetic Energy .................................................................... 102

5.6 Curve-fit of Profiles for Future Use...................................................................... 103

Chapter 6: Conclusions and Recommendations 109

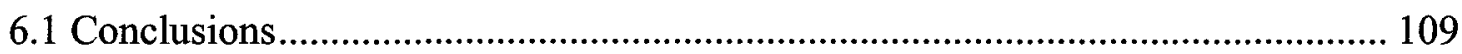

6.2 Recommendations for Future Work............................................................... 110

Appendix A: Uncertainty in Instantaneous Velocity Measurement using PIV 111

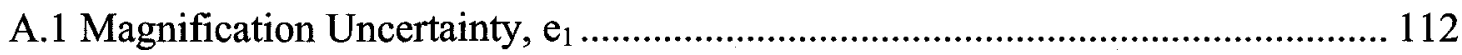

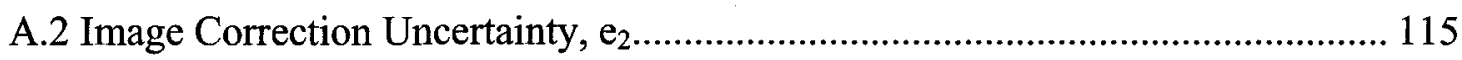

A.3 Non-Zero Laser Pulse Length Uncertainty, $e_{3}$................................................... 116

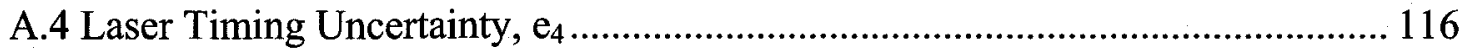

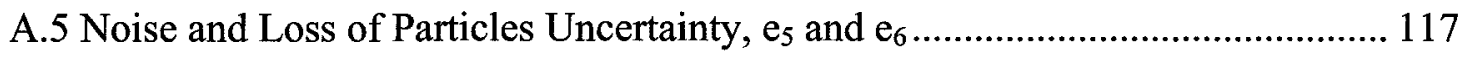

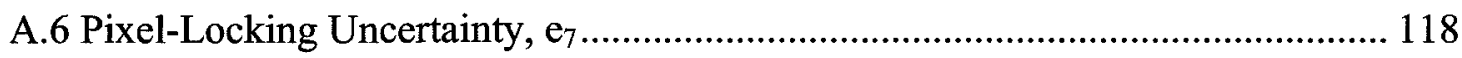

A.7 Velocity Gradient Bias Uncertainty, e e .............................................................. 119

A.8 Traceability of Seed Particles ……………………………………………..... 120

A.9 Total Measurement Uncertainty..................................................................... 123

Appendix B: Uncertainty in Time Delay $\quad 125$

B.1 Time For He:Ne Laser Beam to Travel from Canister to Photodiode .................. 125

B.2 Rise Time of the Photodiode .............................................................................. 125

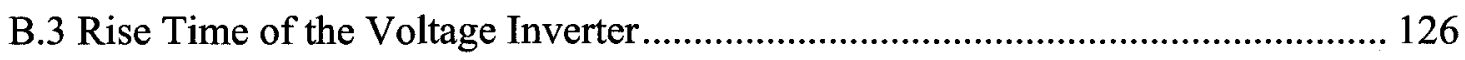

B.4 Time for Signal to Travel from pMDI to PIV Computer...................................... 126

B.5 Resolution of the PIV Computer Timing Card ................................................... 126

B.6 Operation of Metering Valve ........................................................................... 127 
B.6 Maximum Possible Delay

127

Appendix C: RMS Velocity at Selected Times and Locations

128

Appendix D: Velocity at Spacer Outlet

134

Appendix E: Average Turbulent Kinetic Energy

140

Appendix F: Velocity Data Used for Curve Fits

146

References

149 


\section{List of Tables}

Table 3.1 - Factors affecting the choice of PIV interframe time.................................... 28

Table 3.2 - The error associated with the model used to transform pixel coordinates to

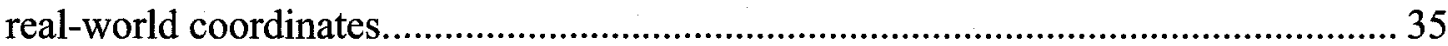

Table 3.4 - Statistical Significance Analysis of Shaking Frequency. ratio-salbutamol HFA. $x=2.5 \mathrm{~mm}, y=0 \mathrm{~mm} .10 \mathrm{~ms}$.

Table 3.5 - Statistical significance of canister lot number on spray velocity at four locations and four time delays. ..................................................................................... 45

Table 3.6 - Canister rotation schedule used to minimize bias error................................... 46

Table 3.7 - Measurement conditions ........................................................................... 47

Table 3.8 - Downstream cutoff locations for geometry-based spray geometry calculation. 61

Table 3.9 - Listing of elemental errors associated with PIV measurements..................... 65

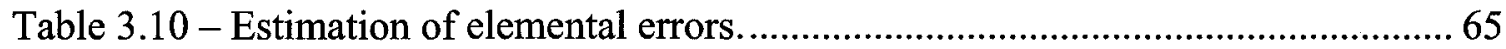

Table 3.11 - Elemental errors associated with data acquisition triggering. ...................... 68

Table 5.1 - The minimum, maximum, and time-averaged spray cone angle and direction. 96

Table 5.2 - Statistical significance between full and empty canister. .............................. 98

Table 5.3 - Statistical significance comparing both brands of canister......................... 100

Table 5.4 - Curve fit parameters for streamwise velocity, $u$, as a function of time. ...... 105

Table 5.5 - Curve fit parameters for vertical velocity, $v$ as a function of time. ............. 106

Table A.1 - The number of vectors possibly affected by velocity gradient bias............ 120

Table A.3 - Measurement uncertainty for selected values of displacement.................... 124

Table C.1 - RMS of streamwise and vertical velocity, in m/s. Ventolin, $1.3 \mathrm{~ms} . . . \ldots . . .1129$

Table C.2 - RMS of streamwise and vertical velocity, in m/s. Ventolin, $2 \mathrm{~ms} . . . \ldots \ldots . . . . . .129$

Table C.3 - RMS of streamwise and vertical velocity, in m/s. Ventolin, $5 \mathrm{~ms} . . . \ldots \ldots \ldots . . .130$ 
Table C. 4 - RMS of streamwise and vertical velocity, in m/s. Ventolin, $10 \mathrm{~ms}$.......... 130

Table C.5 - RMS of streamwise and vertical velocity, in $\mathrm{m} / \mathrm{s}$. Ventolin, $20 \mathrm{~ms}$.......... 131

Table C.6 - RMS of streamwise and vertical velocity, in $\mathrm{m} / \mathrm{s}$. Ventolin, $40 \mathrm{~ms}$.......... 131

Table C.7 - RMS of streamwise and vertical velocity, in $\mathrm{m} / \mathrm{s}$. Ventolin, $60 \mathrm{~ms}$........... 132

Table C.8 - RMS of streamwise and vertical velocity, in $\mathrm{m} / \mathrm{s}$. Ventolin, $80 \mathrm{~ms} . . . \ldots \ldots . .132$

Table C.9 - RMS of streamwise and vertical velocity, in $\mathrm{m} / \mathrm{s}$. Ventolin, $100 \mathrm{~ms}$........ 133

Table F. 1 - Streamwise velocity at $x=1 \mathrm{~mm}$ for various vertical $(y)$ locations and time delays.

Table F. 1 - Vertical velocity at $x=1 \mathrm{~mm}$ for various vertical $(y)$ locations and time delays. 


\section{List of Figures}

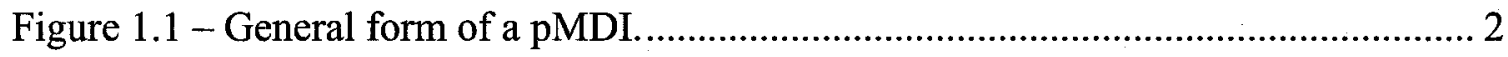

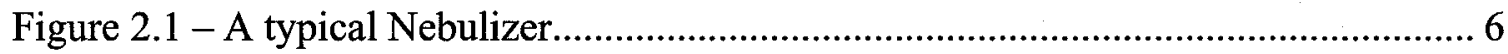

Figure 2.2 - Three common DPI designs. ................................................................. 6

Figure 2.3 - A typical pMDI containing salbutamol sulphate, a common asthma

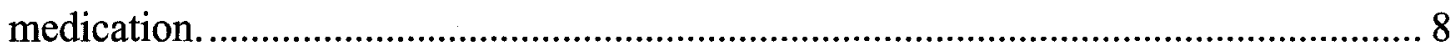

Figure 2.4 - pMDI components..................................................................................... 8

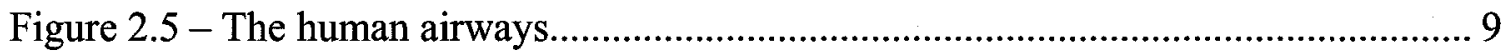

Figure 2.6 - The relative amount of medication deposited at various locations under the optimal conditions of each device. $\mathrm{NEB}=$ Nebulizer. $\mathrm{pMDI} / \mathrm{HC}=\mathrm{pMDI}$ with a holding chamber. Adapted from Fink (2000). ........................................................... 11

Figure 2.7 - Droplet velocity in the centre of the mouthpiece exit plane. Adapted from

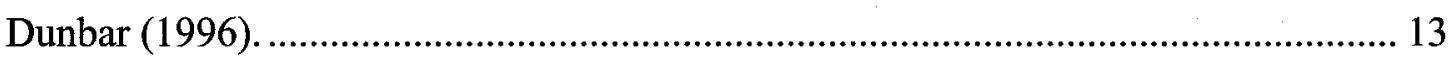

Figure 2.8 - A simple add-on device...................................................................... 17

Figure 2.9-Effect of spacer length and diameter on available respirable mass of medication. Adapted from Barry and O'Callaghan (1995a). ........................................ 18

Figure 3.1 - Mechanism to actuate the pMDI. ............................................................... 20

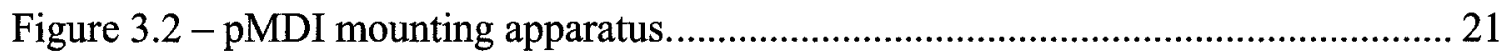

Figure 3.3 - Schematic of the apparatus used to force an air flow, seeded with olive oil particles, through the pMDI casing............................................................................ 23

Figure 3.4 - Photograph of the collar used to seal the gap between the pMDI casing and the tee.

Figure 3.5 - Photograph of a pMDI inserted into the plastic tee via the collar. 24

Figure 3.6 - The laser beam passes through a plano-concave lens followed by a symmetrical convex lens, forming a sheet $100 \mathrm{~mm}$ high 
Figure 3.7 - The laser is positioned so that the measurement region is at the waist (the

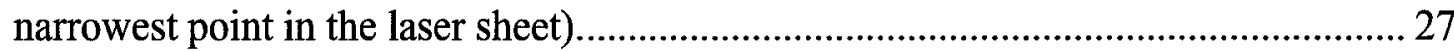

Figure 3.8 - Timing diagram showing the effective exposure time of the PIV camera system.

Figure 3.9 - The image of the calibration plate used to calibrate the Imager Pro 2M. ... 31

Figure 3.10 - The calibration plate mounting apparatus................................................ 32

Figure 3.11 - The Imager Pro 2M (PIV camera) mounting apparatus. ............................ 33

Figure 3.12 - The fields of view for pMDI mouthpiece outlet measurement cases, (a) without and (b) with and add-on spacer device.

Figure 3.13 - The location of the hole where the He:Ne laser passes to trigger the data acquisition system. 36

Figure 3.14 - Experimental setup 39

Figure 3.15 - Average velocity along spray centreline for the first 25 actuations following canister shaking. ratio-Salbutamol HFA, $10 \mathrm{~ms}$.

Figure 3.16 - Minimum and maximum velocity, as well as average velocity and its standard deviation, along the spray centreline for the first 10 actuations following canister shaking. ratio-Salbutamol HFA, $10 \mathrm{~ms}$.

Figure $3.17-a)$ The initial pass searches $1 / 2$ the IW size in all directions and returns an approximate velocity vector. b) The result of this cross-correlation calculation, as well as those in surrounding IWs, is used as a reference vector field for the next pass, improving the signal to noise ratio. 50

Figure 3.18 - A example correlation map. Fitting the correlation map to a Gaussian function in the vicinity of the peak allows the peak location (indicated by the arrow) to be determined with sub-pixel resolution.

Figure 3.19 - IWs are overlapped to increase the resolution of the resulting vector flow field. 53

Figure 3.20 - A sample instantaneous vector velocity field before post-processing........ 56 Figure 3.21 - Spurious vectors remaining after peak-ratio rejection. A vector was removed at (a). The vector at (b) will be removed by the velocity-rejection step. The 
vector at (c) will be removed by the small-groups step. The vectors at (d) will not be removed.

Figure 3.22 - The number of vectors remaining in the measurement region after postprocessing. Ventolin, open air, $1.3 \mathrm{~ms}$.

Figure 3.23 - The locations of the peak and half-peak velocities at four streamwise locations, as well as the location of the nozzle, are fitted linearly. The spray direction and angle are then calculated using the linear fits. 59

Figure 3.24-A sample image showing the downstream cutoff location for spray at early time delays. ratio-Salbutamol HFA, $2 \mathrm{~ms}$.

Figure 3.25 - The Geometry algorithm places the spray edge on a given row such that half of the pixels showing a spray are inside the spray and half are outside the spray. The centerline is calculated to be midway between the edge lines. 62

Figure 3.26 - The apparatus used to mount the add-on device. ......................................... 63

Figure 3.27 - The uncertainty in velocity expressed as a function of velocity. ................ 67

Figure 3.28 - Normalized running average for streamwise velocity, $u$............................ 69

Figure 3.29 - Histograms of measurements of streamwise velocity, $u$, at two locations and three time delays. Overlaid with a corresponding normal distribution for comparison. 71

Figure 4.1 - Two images of the spray at a time delay of $1.7 \mathrm{~ms}$. 73

Figure 4.2 - Histogram of tip penetration taken at a time delay of $1.7 \mathrm{~ms}$, overlaid with a normal distribution having the same mean and standard deviation. 74

Figure 4.3 - Large differences in velocity are seen between two actuations, selected at random. Ventolin, open air, $2 \mathrm{~ms}$. 75

Figure 4.4 - Average vector velocity flow field. Ventolin, open air, (a) 1.3 and (b) $2 \mathrm{~ms}$.

Figure 4.5 - Average vector velocity flow field. Ventolin, open air, (a) 5 and (b) $10 \mathrm{~ms}$.

Figure 4.6 - Average vector velocity flow field. Ventolin, open air, (a) 20 and (b) $40 \mathrm{~ms}$. 
Figure 4.7 - Average vector velocity flow field. Ventolin, open air, (a) 60 and (b) $80 \mathrm{~ms}$.

Figure 4.8 - Average vector velocity flow field. Ventolin, open air, $100 \mathrm{~ms}$................. 80

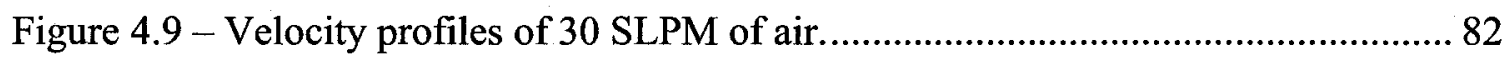

Figure 4.10 - Direction of Dean vortices, if present. View into mouthpiece................... 82

Figure 4.11 - Comparison of vertical profiles of streamwise spray velocity with and without a 30 SLPM air flow. Ventolin, (a) 1.3 and (b) 2 ms. 83

Figure 4.12 - Comparison of vertical profiles of streamwise spray velocity with and without a 30 SLPM air flow. Ventolin, (a) 5 and (b) $10 \mathrm{~ms}$.

Figure 4.13 - Comparison of vertical profiles of streamwise spray velocity with and without a 30 SLPM air flow. Ventolin, (a) 20 and (b) $40 \mathrm{~ms}$. 84

Figure 4.14-Comparison of vertical profiles of streamwise spray velocity with and without a 30 SLPM air flow. Ventolin, (a) 60 and (b) $80 \mathrm{~ms}$. 85

Figure 4.15 - Comparison of vertical profiles of streamwise spray velocity with and without a 30 SLPM air flow. Ventolin, $100 \mathrm{~ms}$. 85

Figure 4.16-Vertical profiles of streamwise spray velocity with and without the Aerochamber. Ventolin, (a) 1.3 and (b) $1.7 \mathrm{~ms}$.

Figure 4.17 - Vertical profiles of streamwise spray velocity with and without the Aerochamber. Ventolin, (a) 2 and (b) $5 \mathrm{~ms}$. 88

Figure 4.18 - Vertical profiles of streamwise spray velocity with and without the Aerochamber. Ventolin, (a) 10 and (b) $20 \mathrm{~ms}$. 88

Figure 4.19-Vertical profiles of streamwise spray velocity with and without the Aerochamber. Ventolin, (a) 40 and (b) $60 \mathrm{~ms}$.

Figure 4.20-Vertical profiles of streamwise spray velocity with and without the Aerochamber. Ventolin, (a) 80 and (b) $100 \mathrm{~ms}$.

Figure 5.1 - Temporal evolution of the average streamwise velocity during the first $120 \mathrm{~ms}$ of the spray event. Ventolin only. 92

Figure 5.2 - Temporal evolution of the average streamwise velocity during the first $120 \mathrm{~ms}$ of the spray event. Comparing the peak velocity for a given streamwise location with the velocity on the centreline. Ventolin only. 93 
Figure 5.3 - Comparison of the spray direction calculated using both calculation methods, as well as the effect of the 30 SLPM co-flow.

Figure 5.4 - Comparison of the spray cone angle calculated using both calculation methods, as well as the effect of the 30 SLPM co-flow. 96

Figure 5.5 - Average turbulence kinetic energy calculated using equation 5.2. ratiopharm, $60 \mathrm{~ms}$ 103

Figure 5.6 - Vertical profiles of streamwise velocity and associated curve fits. 107

Figure 5.7 - Vertical profiles of vertical velocity and associated curve fits. 108

Figure A.1 - Diagram of image and object planes. 114

Figure A.2 - A histogram of (vector displacement $\bmod 1$ ), in pixels 118

Figure D.1 - The field of view used for PIV measurements at the outlet of the add-on device. 134

Figure D.2 - Average vector velocity flow field. Ventolin, spacer outlet, $2.5 \mathrm{~ms} . \ldots \ldots . .135$

Figure D.3 - Average vector velocity flow field. Ventolin, spacer outlet, $5 \mathrm{~ms}$........... 135

Figure D.4 - Average vector velocity flow field. Ventolin, spacer outlet, $7.5 \mathrm{~ms}$........ 136

Figure D.5 - Average vector velocity flow field. Ventolin, spacer outlet, $10 \mathrm{~ms}$......... 136

Figure D.6 - Average vector velocity flow field. Ventolin, spacer outlet, $12.5 \mathrm{~ms}$..... 137

Figure D.7 - Average vector velocity flow field. Ventolin, spacer outlet, 15 ms. ........ 137

Figure D. 8 - Average vector velocity flow field. Ventolin, spacer outlet, $20 \mathrm{~ms}$......... 138

Figure D.9 - Average vector velocity flow field. Ventolin, spacer outlet, $40 \mathrm{~ms}$......... 138

Figure D.10 - Average vector velocity flow field. Ventolin, spacer outlet, $60 \mathrm{~ms}$....... 139

Figure D.11 - Average vector velocity flow field. Ventolin, spacer outlet, $100 \mathrm{~ms} . . . .139$

Figure E.1 - Turbulent kinetic energy. ratiopharm, 1.3 ms............................................. 141

Figure E.2 - Turbulent kinetic energy. ratiopharm, $1.7 \mathrm{~ms}$.......................................... 141

Figure E.3 - Turbulent kinetic energy. ratiopharm, 2 ms......................................... 142

Figure E.4 - Turbulent kinetic energy. ratiopharm, 5 ms............................................. 142

Figure E.5 - Turbulent kinetic energy. ratiopharm, $10 \mathrm{~ms}$....................................... 143

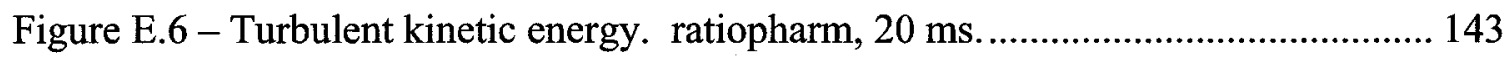

Figure E.7 - Turbulent kinetic energy. ratiopharm, 40 ms......................................... 144

Figure E. 8 - Turbulent kinetic energy. ratiopharm, $60 \mathrm{~ms}$............................................ 144 


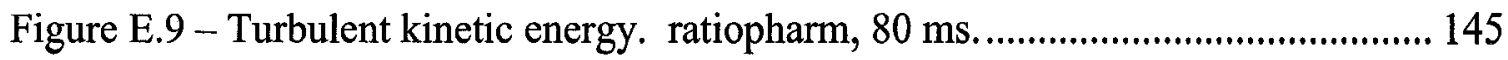

Figure E.10 - Turbulent kinetic energy. ratiopharm, 100 ms......................................... 145 


\title{
Nomenclature
}

\author{
Variables \\ C \\ M \\ $n$ \\ $u$ \\ $v$ \\ $\vec{V}$ \\ $x$ \\ $y$ \\ $z$ \\ $\mathrm{d}_{\mathrm{pix}}$ \\ $\mathrm{d}_{\tau}$ \\ $d x$ \\ $d y$ \\ $I_{1} \& I_{2}$ \\ $n_{1} \& n_{2}$ \\ $s_{1} \& s_{2}$ \\ $t_{\mathrm{t}}$ \\ Correlation function \\ Magnification factor between object and image plane \\ Interrogation window size \\ Streamwise component of velocity \\ Vertical component of velocity \\ Vector velocity \\ The streamwise direction (origin at the pMDI mouthpiece plane) \\ The vertical direction (origin at the pMDI nozzle) \\ The transverse direction (origin on the pMDI axis of symmetry) \\ Side length of a pixel \\ Imaged diameter of a particle \\ Shift in the $x$-direction \\ Shift in the $y$-direction \\ Image intensity in image 1 or 2 \\ Number of measurements used to calculate Student's t-score \\ Standard deviation of a sample \\ Time elapsed since the data acquisition system was triggered
}


$\bar{x}_{1} \& \bar{x}_{2}$

$\delta(\mathrm{e})$

$\Delta t$

$\overrightarrow{\Delta X}$

Acronyms

CCD

CFC

CFD

DPI

ETA

FFT

FOV

FPF

FWHM

HFA

IW

MMAD

PDA

PDPA

PIV

pMDI

psig
Mean value of a sample used to calculate Student's t-score

Used to represent the uncertainty of a given quantity, e

Interframe time

Displacement, measured in the image plane

Charge-coupled device

Chlorofluorocarbon

Computational fluid dynamics

Dry powder inhaler

Extrathoracic airways

Fast Fourier transform

Field of view

Fine particle fraction

Full-width, half-maximum

Hydrofluoroalkane

Interrogation window

Mass-median aerodynamic diameter

Phase Doppler anemometry

Phase Doppler particle analysis

Particle image velocimetry

Pressurised metered-dose inhaler

Gage pressure in units of pounds per square inch

xviii 
SLPM

Standard litres per minute; litres of air at 101325 pa and $0 \mathrm{C}$, per minute

Medical/Latin Terminology

Distal

An anatomical direction. Within the ETA this refers to points further downstream, i.e. away from the mouth or nose

In vivo

An experimental medical study involving live patients

In vitro

An experimental medical study involving anatomical models

Oropharyngeal

Relating to the oropharynx

Oropharynx

The portion of the neck and throat (pharynx) situated behind the mouth

Placebo

In pMDIs, a canister that contains propellant but no medication

Proximal

An anatomical direction. Within the ETA this refers to points further upstream, i.e. closer to the mouth or nose. 


\section{Chapter 1: Introduction}

\subsection{Motivation}

Asthma is a serious and potentially fatal chronic lung disease. Of the estimated 2.7 million asthmatic Canadian adults and children (ages 4 years and over), 287 died of asthma in 2003 (Health Canada, 2006). Asthma and other respiratory diseases are commonly treated with inhaled medications, which are frequently delivered orally via a pressurised Metered-Dose Inhaler (pMDI).

Though pMDIs have been in existence for more than 50 years, their general form remains similar to that depicted in Figure 1.1. The efficacy of pMDIs in delivering drugs deep into the lungs did not noticeably improve during the first 40 years, remaining near $10 \%$ (Fink 2000). It is generally accepted that improved efficacy will allow for similar treatment with fewer side effects at a lower cost to the patient. Recent increases in pMDI efficacy are also allowing pMDIs to be used in the treatment of diseases such as diabetes, where the systemic side effects attributed to particle deposition in the mouth and throat previously rendered the treatment unsafe. 


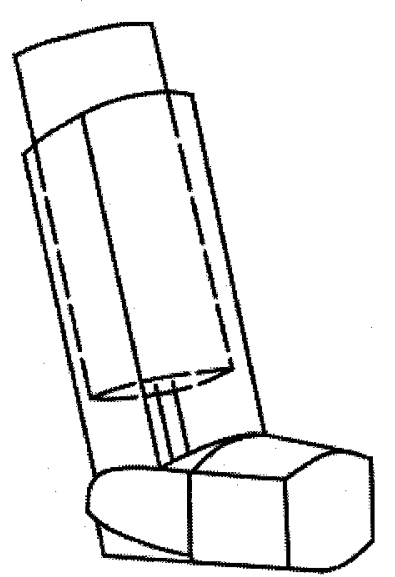

Figure 1.1 - General form of a pMDI.

\subsection{Thesis Objectives}

The work presented herein has three main objectives. The first objective is to increase the understanding of the spray emitted by a pMDI, especially concerning spray velocities in the region downstream of the pMDI mouthpiece. Limited existing data do not provide a clear picture of spatial and time variations in spray velocities.

The second objective is to compare the velocities measured in some of the many configurations under which pMDIs are used, and to describe their similarities and differences. Previous studies have separately considered use of pMDIs by children (Berg et al., 1998; Finlay and Zuberbuhler, 1999), by uncoordinated patients (Newman et al., 1991; Fink et al., 1998), and with various add-on devices (Vidgren et al., 1987; Newman and Newhouse, 1996). By comparing spray velocities and spreading angles under different configurations, it is hoped that similarities can be observed such that future researchers can more broadly interpret results from specific experiments as well as identify critical differences. 
The third and final objective is to provide a parametric representation of the spray velocity at the mouthpiece outlet, to facilitate future numerical simulations involving pMDI sprays.

\subsection{Approach}

The current research uses a measurement technique called particle image velocimetry (PIV) to determine the displacement of particle images between two instants in time. One PIV image pair is obtained per pMDI actuation, and 100 image pairs are obtained at each of ten different times after pMDI actuation. Statistical cross-correlation is performed on each image pair to produce a vector velocity field with a spatial resolution of $0.4 \mathrm{~mm}$. Each set of 100 vector velocity fields was averaged to produce mean velocity fields at each of the ten time delays, achieving the first objective.

To compare different conditions, new sets of 100 image pairs at ten time delays were obtained under the following conditions: i) with a pMDI canister manufactured by a different company; ii) with an air flow of 30 SLPM (standard litres per minute, refers to litres per minute at $101325 \mathrm{~Pa}$ and $0^{\circ} \mathrm{C}$ ) being forced through the pMDI casing to simulate steady patient inhalation; and iii) with the pMDI mouthpiece issuing into a commonly-used add-on device while an air flow of 30 SLPM simulating patient inhalation is forced through the pMDI casing and the add-on device. Analysis of the velocities measured under these conditions, as well as comparison between the velocities measured using a full canister and an empty canister, fulfills the second objective.

To fulfill the third and final objective of the thesis, vertical profiles of streamwise and vertical velocity were extracted from the vector velocity flow fields as close to the 
mouthpiece as possible. These profiles were then fitted to parametric curves, allowing the velocity profiles to be inputted directly into future numerical simulations.

\subsection{Overview of Thesis}

The thesis is organized into four major sections, represented by chapters 2 through 5 . Chapter 2 serves as an introduction to pMDIs and provides a review of the literature concerning pMDIs and their efficacy. Chapter 3 provides a detailed description of the experimental apparatus used in the current research, as well as the procedures followed to acquire reliable data. Chapter 4 presents the major experimental results, beginning with a detailed discussion of the results from a single experimental condition, followed by comparisons of subsequent cases to this first case. These results are analysed and discussed in Chapter 5, before major conclusions are summarized in Chapter 6. 


\section{Chapter 2: Background and Literature Review}

\subsection{Introduction}

The purpose of this chapter is to provide and analyse information related to this thesis. It begins with a brief description of the different devices used in inhalation therapy, followed by a thorough description of pMDIs. The chapter then reviews previous work attempting to quantify the efficacy of pMDIs, followed by a description of the spray. Finally, add-on devices, (loosely classified as spacers or holding chambers depending on their volume), which are commonly used in conjunction with pMDIs, are introduced followed by a discussion of their efficacy.

A number of devices are currently used to deliver aerosolized medication to the lungs. The most common are nebulizers, dry-powder inhalers and pressurised metered-dose inhalers.

\subsection{Nebulizers and Dry Powder Inhalers}

Nebulizers (also called atomizers) pump air through a liquid medication, creating a mist. This fine mist tracks the flow through the airways well, with minimal deposition upstream of the lungs. This makes nebulizers ideal for patients with severe respiratory problems who have trouble breathing on their own. As shown in Figure 2.1, they are too 
large to be portable, require a small compressor, and are generally reserved for hospital use.

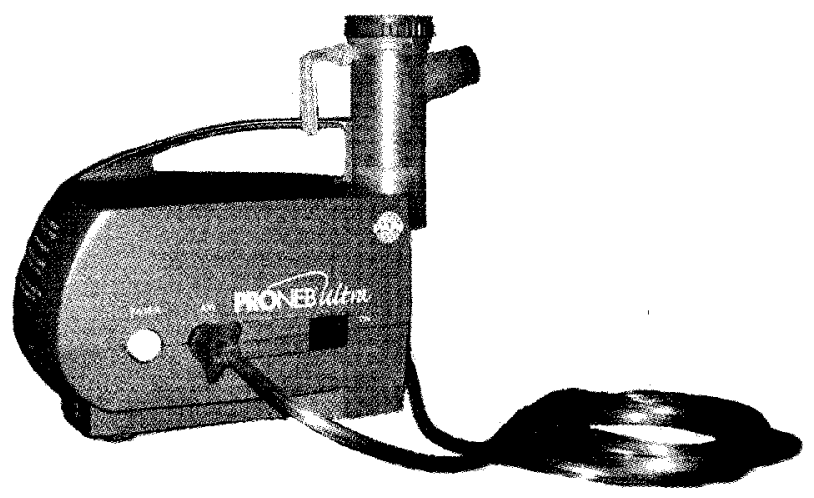

Figure 2.1 - A typical Nebulizer.

Dry-powder inhalers (DPIs) are small, portable devices originally designed to eliminate some of the problems associated with pMDIs. A typical DPI might contain 30 individually sealed doses of medication in powder form. A number of different designs are available, some of which are shown in Figure 2.2.

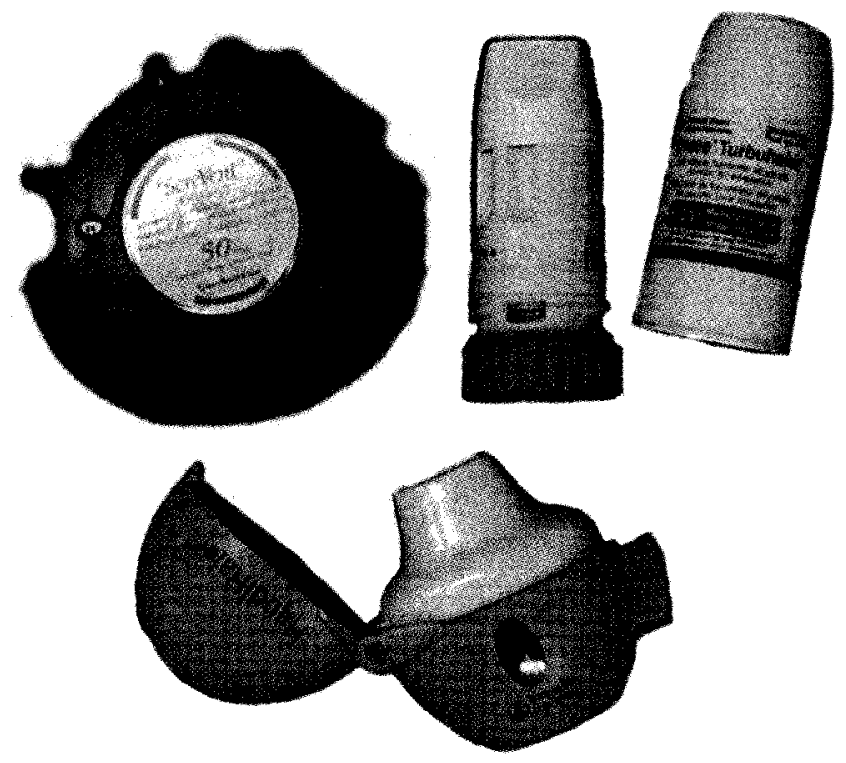

Figure 2.2 - Three common DPI designs. 
The user primes the device by actuating a lever and then inhales from the mouthpiece when he or she is ready, eliminating the need for user coordination. With some designs, the priming action also actuates a counter so the user knows how many doses remain. DPIs are generally more expensive than pMDIs, and they are less effective in patients with obstructed airways or young children who may be unable to achieve the high inspiratory flow rates required to activate them. Their efficacy is also reduced when used in humid environments (Fink, 2000).

\subsection{Pressurised Metered-Dose Inhalers}

The pressurised metered-dose inhaler is a widely-used medical device designed to deliver aerosolized medication deep into the lungs. A pMDI manufactured by GlaxoSmithKline Inc. is shown in Figure 2.3. A typical pMDI consists of a canister and a casing. The canister contains a pressurised mixture of medication and propellant, as shown in Figure 2.4. The majority of pMDIs currently in use, including those used in this research, contain hydrofluoroalkane-134a (HFA) as a propellant.

As described by Newman (2005), one end of the canister contains a metering mechanism, ending in the outlet, or valve stem. Often the medication is coated with a surfactant to prevent blockage of the metering mechanism. The canister is then inserted into the casing, where the union of the valve stem and the actuator stem form an expansion chamber. A small hole in the expansion chamber, usually between 0.25 and $0.5 \mathrm{~mm}$ in diameter, acts as a nozzle. When the pMDI is actuated by squeezing the canister, the medication/surfactant/propellant mixture passes through the metering valve, out the valve stem, into the expansion chamber and out the nozzle, forming a respirable 
spray. The flow leaving the nozzle is turbulent, having a Reynolds number (based on nozzle diameter) of about 100000 . For a more detailed description of pMDI design see Finlay (2001) or Dunbar (1996).

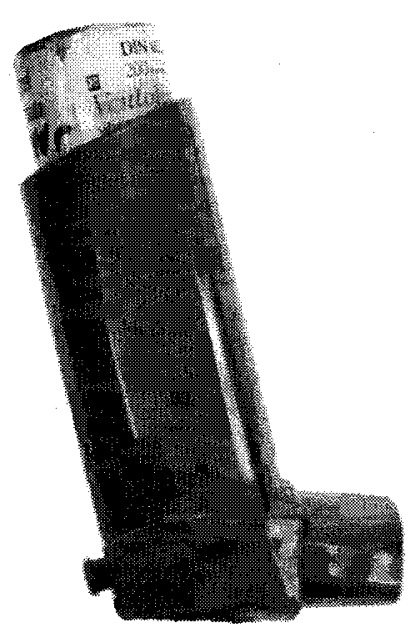

Figure 2.3 - A typical pMDI containing salbutamol sulphate, a common asthma medication.

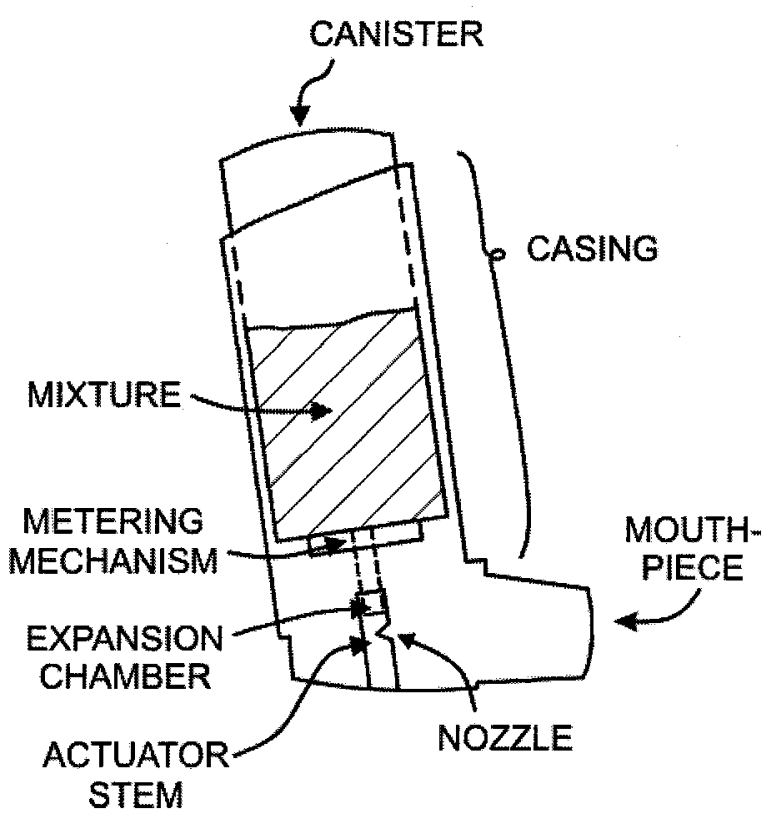

Figure $2.4-$ pMDI components.

\subsection{1 pMDI Efficacy}

Since the primary goal of most pMDIs is to deliver medication to the lungs, the most general definition of pMDI efficacy is the ratio of the amount of medication that arrives in the alveoli to the amount of medication that was emitted by the pMDI:

$$
\text { efficacy }=\frac{\text { mass of medication in alveoli }}{\text { mass of pMDI nominal dose }}
$$


Described this way, pMDI efficacy is impossible to measure directly when considering the complexities of the human airway as shown in Figure 2.5. The closest approximation to this ideal definition is obtained during in vivo studies of pMDIs employing radiolabeled particles, as described by Clarke et al. (1992). Patients use these devices as they would any other pMDI. Scintigraphic images are then taken with gamma cameras, and the images are analysed to approximate the amount of radiolabeled material present in the patient, as described by Newman (1993). This is used to approximate the total amount of medication delivered, and compared to the nominal dose of the pMDI being tested.

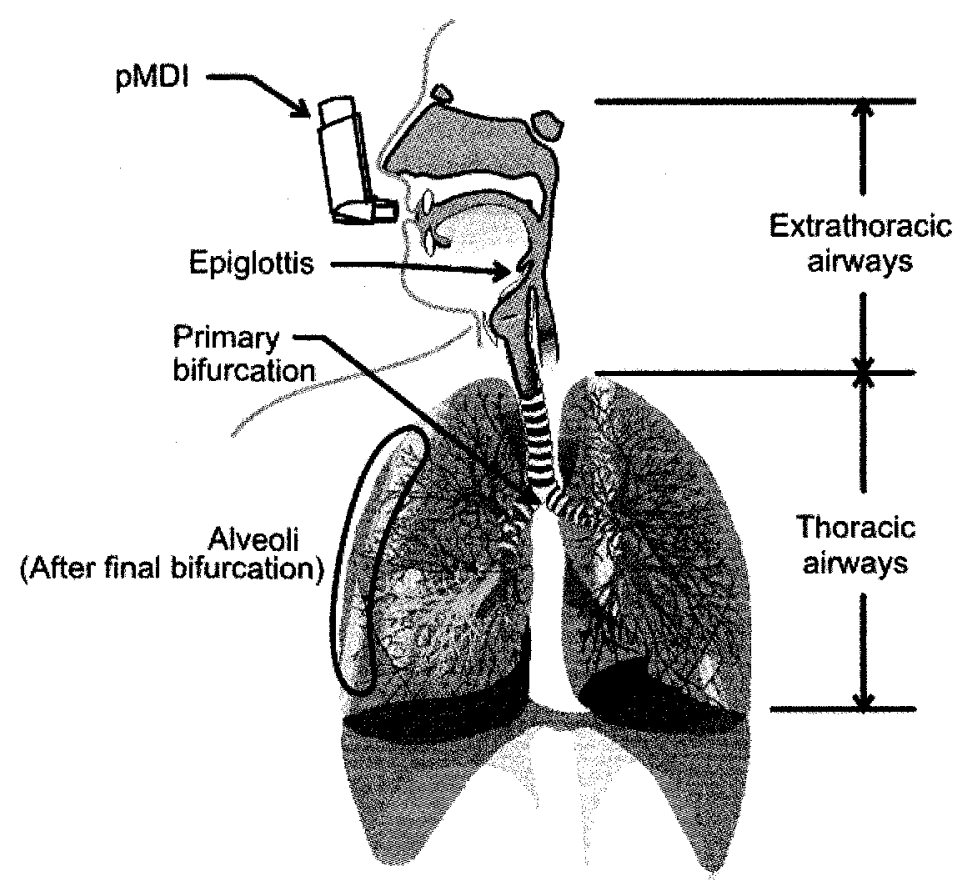

Figure 2.5 - The human airways. 
When studying pMDIs in vitro, the lack of a single standard airway or lung model necessitates a simpler definition of pMDI efficacy. For this reason, some researchers have used the idea of the fine particle fraction (FPF), defined as the fraction of mass contained within particles having a mass median aerodynamic diameter (MMAD) below a given cutoff diameter. Aerodynamic diameter is the diameter of a sphere of unit density having the same aerodynamic properties as the particle in question. For aerosol particles, the aerodynamic property of interest is the terminal settling velocity, commonly defined using the Stokes number. It is generally considered that the mass represented by the FPF will reach the lungs (Bates et al., 1966). Different researchers have used a different cutoff MMAD; often the choice depends on the equipment available for particle sizing. Graham (1995) suggested that a "fine respirable fraction" for salbutamol particles was represented by particles having an MMAD between 1.1 and $5.8 \mu \mathrm{m}$. More recent research often uses a cutoff diameter of $4.7 \mu \mathrm{m}$ (e.g. Barry and O'Callaghan (1999), Berg et al. (1998), Tarara et al. (2004)). The definition of a FPF allows for the study and optimization of pMDI spray without careful consideration of the extrathoracic geometry.

More detailed studies have attempted to recreate the flow through the extrathoracic airways (ETA), using either an idealized ETA such as that proposed by Stapleton (2000) or realistic models taken from casts of cadavers (e.g. Cheng et al., 2001) or scans of live patients (e.g. Rahmatalla et al., 2002). This allows the measurement of particle deposition in various regions of the ETA, such as the mouth, the back of the throat (oropharynx) or more downstream (distal) regions such as the epiglottis or primary lung bifurcations. An absolute filter placed at the most distal location of the model will 
capture particles assumed to arrive and remain in the lungs, representing the respirable mass of medication.

The various methods used to measure and quantify pMDI efficacy produce varied results, under both similar and differing experimental conditions. Even within the review presented by Fink (2000), it is obvious that the efficacy of pMDIs varies greatly depending on the specific conditions of inhalation. Figure 2.6 uses data gathered by Fink (2000) to compare the amount of medication deposited at various locations by patients using various devices. It is important to compare not only the amount of medication deposited in the lungs, but also the amount of oropharyngeal deposition, which generally leads to unwanted systemic side effects. Thus for a patient capable of using any of the devices, the best choice would seem to be a pMDI with a holding chamber.

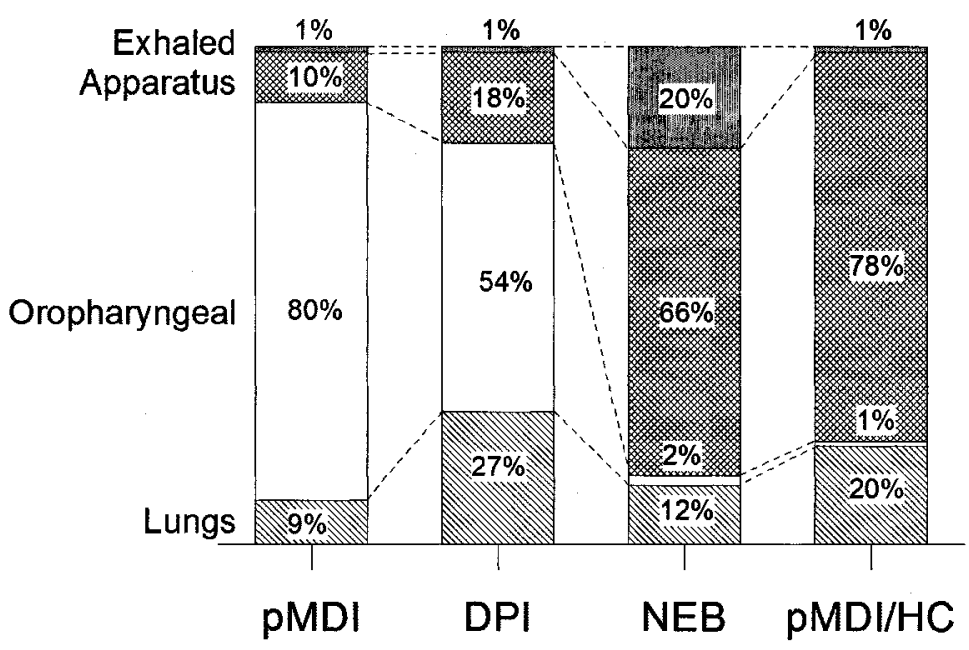

Nominal Dose $\quad 200 \mu \mathrm{g} \quad 200 \mu \mathrm{g} \quad 2500 \mu \mathrm{g} \quad 200 \mu \mathrm{g}$

Figure 2.6 - The relative amount of medication deposited at various locations under the optimal conditions of each device. $\mathrm{NEB}=$ Nebulizer. $\mathrm{pMDI} / \mathrm{HC}=\mathrm{pMDI}$ with a holding chamber. Adapted from Fink (2000). 
Unfortunately patients vary greatly in terms of their ability to coordinate breathing with pMDI actuation, inhalatory strength (necessary for proper DPI inhalation), and persistence in continued use of nebulizers or bulky holding chambers, especially in urgent use situations. Though the FPF criterion simplifies in vitro analysis, it cannot take these factors into consideration. To quantify the benefits or drawbacks of these varied usage conditions, as well as other areas of interest such as the effects of a change in the spray angle relative to the patient (Fadl et al., 2007) or regional targeting (Kleinstreuer et al., 2007), extensive experimentation leading to comprehensive numerical simulation is required.

\subsubsection{Characterisation of pMDI Spray Velocities}

Before pMDI sprays can be simulated, either numerically or experimentally, the true spray must be properly characterized. Dunbar and coworkers (1996, 1997a, 1997b) provided a thorough theoretical and experimental analysis of the pMDI spray. They made measurements of velocity at discrete points in the flow-field using phase-Doppler particle analysis (PDPA), which allowed droplet velocity, size (assuming spherical droplets), and concentration to be measured. This work was done during the search for a suitable propellant that would replace chlorofluorocarbon-based propellants in use at the time. For this reason, their work was done on custom-manufactured canisters containing only an HFA-placebo formulation.

An important aspect of their work was the comparison of the measured spray velocity with numerically-simulated spray velocities. The measured and simulated velocity 
profile at the centre of the mouthpiece, in the mouthpiece plane, is reproduced in Figure 2.7.

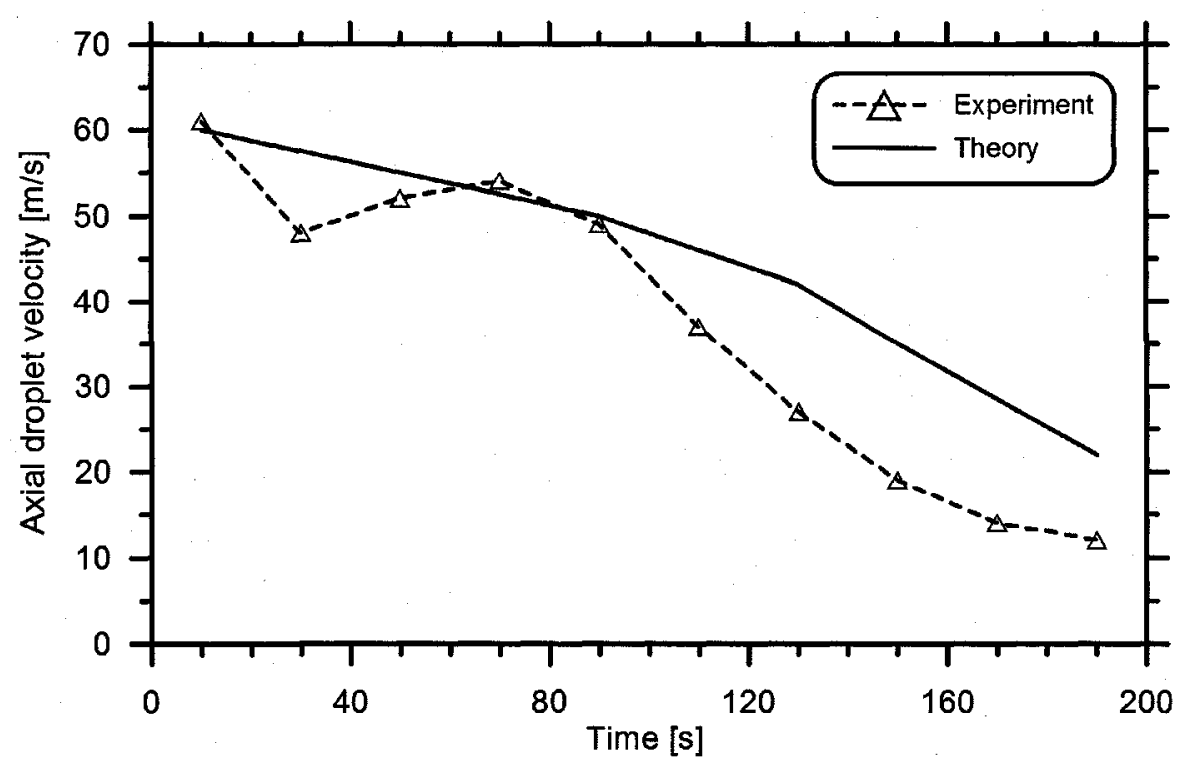

Figure 2.7 - Droplet velocity in the centre of the mouthpiece exit plane. Adapted from Dunbar (1996).

The numerical study used the discrete droplet model, describing the gas phase and the dispersed liquid phase separately. Momentum exchange and heat and mass transfer between the phases are accomplished through the use of source terms. Discrete parcels representing many droplets with similar properties were used to reduce computational cost. The eddy interaction model of Gosman and Ioannides (1981) was used to model turbulent dispersion of the droplets. The continuous gas phase used the high-Reynolds number k- $\varepsilon$ turbulence model of Launder and Spalding (1972). A two-dimensional axisymmetric structured grid consisting of 70 nodes in the streamwise direction and 25 nodes in the vertical direction was used. 
While the experimental results showed two local velocity maxima in time, the numerical results yielded only one. It was hypothesized that the excessive spray density in the near-orifice region of the spray was a major source of error. The author decided that measurement dropout in dense spray regions such as this one had biased the results and concluded that the dip in velocity before the second maximum was an artefact of experimental error. In contrast, Phase-Doppler anemometry measurements of a placebo pMDI containing only HFA-227 propellant performed by Wigley et al. (2002) clearly show a second velocity maximum in time, agreeing with the experimental results of Dunbar and coworkers.

Kakade et al. (2007) used a two-component laser Doppler anemometry / PDA system to measure actuation-averaged velocities of the pMDI spray, as part of their investigation of a novel pMDI actuator system, referred to as the vortex nozzle actuator. In testing and optimizing their design, they measured spray velocities of both the vortex nozzle actuator spray and the spray emitted from a Proventil-brand salbutamol pMDI. The results from the Proventil-brand salbutamol pMDI show a peak actuation-averaged velocity of $13.5 \mathrm{~m} / \mathrm{s}$ located between 3 and $7 \mathrm{~mm}$ below the horizontal $(x z)$ plane, extending $2.5 \mathrm{~mm}$ left and right of the central vertical (sagittal) plane. Due to the actuation-averaged nature of these results, it is not possible to compare directly to the work of Dunbar and coworkers (1996, 1997a, 1997b).

An exhaustive search of the open literature of the past 15 years reveals no other detailed measurements of pMDI spray velocities using HFA- or CFC-based propellants. Because this research is concerned primarily with HFA-based pMDIs, a 
less-comprehensive search of the relevant literature prior to 1993 was performed. No relevant pMDI spray velocity measurement data were found.

\subsubsection{Numerical Studies}

In recent years, several researchers have attempted to use computational fluid dynamics (CFD) to model air flow as well as the transport and deposition of aerosols in the extrathoracic airway. Heenan et al. (2004), Jin et al. (2007), Farkas et al. (2006), and Takano et al. (2006) all partially decoupled the particle transport problem by first simulating the air flow and subsequently tracking the simulated release of particles within the calculated fluid domain. Whereas the first three works considered steady flow, Takano et al. (2006) simulated the flow field and particle deposition during unsteady breathing, using breathing waveforms to represent three different inhalation patterns. However unlike with pMDI inhalation, the particles were released with a zero slip boundary condition. In principle, this approach could be extended to the modeling of pMDI sprays by releasing particles with appropriate initial velocities, but this has been limited by the lack of detailed data to effectively model the time-dependent velocity boundary conditions of the pMDI spray.

Heenan et al. (2003) produced and compared experimental and CFD results of air flow in an idealized human oropharynx. They studied three inlet velocity profiles each at volume flow rates of 15,30 , and 90 SLPM. Slightly different inlet velocity profiles were used to determine their effect on the resulting flow field. These three different inlet conditions produced velocity profiles near the oropharynx that varied from three to six 
percent of the ideal plug flow profile at that point. It could be assumed that the effects of a transient boundary condition would be much more significant.

Kleinstreuer et al. (2007) attempted the most comprehensive simulation of the pMDI flow to date. Rather than numerically releasing droplets into a previously calculated flow-field, they used a different approach where models were used to simulate droplet breakup and evaporation. A Reynolds-averaged Navier-Stokes simulation with a low-Reynolds number k- $\omega$ turbulence model was used for the air flow, and the effects of turbulence were neglected when calculating droplet trajectories. The flow was modeled through a pMDI, into a generic spacer and subsequently through a simulated human upper airway. The simulated spray velocities were validated against the work of $\mathrm{Wu}$ et al. (1984) and Hiroyasu and Kadota (1974), both of which studied diesel-fuel sprays. It is presumed that no comparison was made with detailed pMDI spray velocity data because none currently exists in the literature.

Using this model, Kleinstreuer et al. (2007) performed simulations with HFA-propellant properties using a nozzle diameter of $0.25 \mathrm{~mm}$ and with CFC-propellant properties using nozzle diameters of 0.25 and $0.5 \mathrm{~mm}$. The CFC-propelled pMDI had a simulated lung deposition of only 5.2 percent with the larger nozzle. Decreasing the nozzle diameter improved this to 23.2 percent, still less than the 46.6 percent simulated lung deposition achieved using the HFA-propelled pMDI with the smaller orifice diameter. These results are a further indication of the critical role of spray velocity boundary conditions on simulating delivery of medical aerosols. 


\subsection{Add-on Devices}

Many devices have been used in conjunction with pMDIs over the years in an effort to increase their efficacy. The simplest add-on devices are tubes: the pMDI is actuated into one end while the user inhales from the other, as in Figure 2.8. The success of an add-on device depends largely on geometry, as demonstrated by the work of Barry and O’Callaghan (1995a), shown in Figure 2.9.

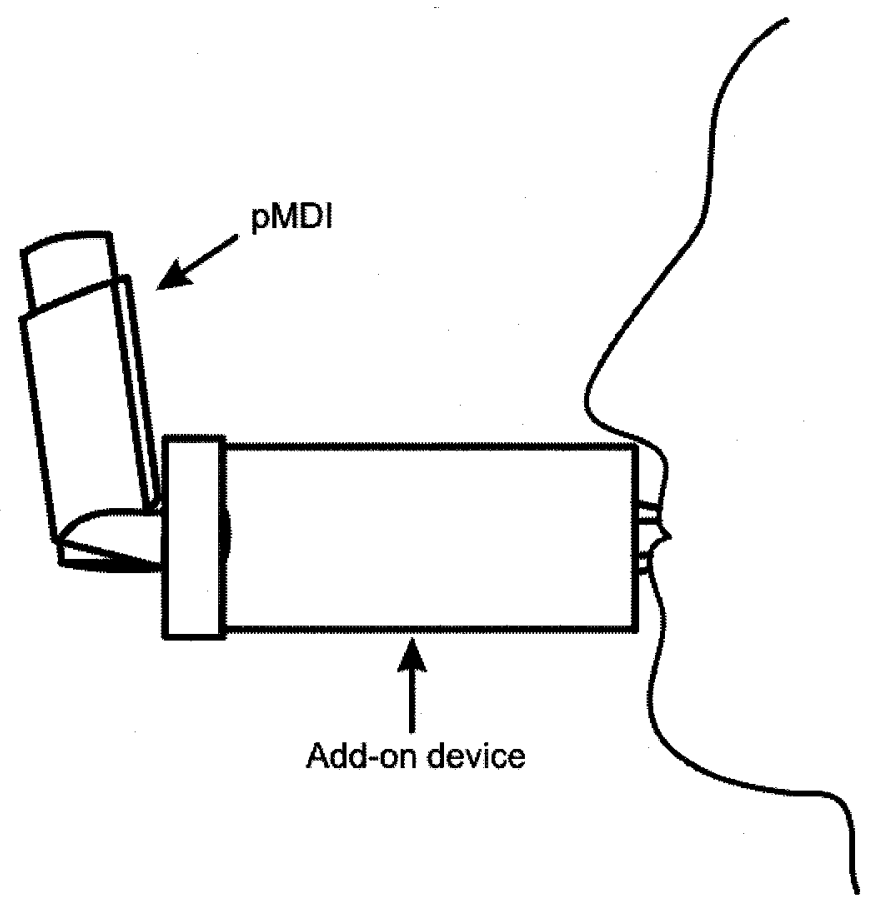

Figure 2.8 - A simple add-on device. 


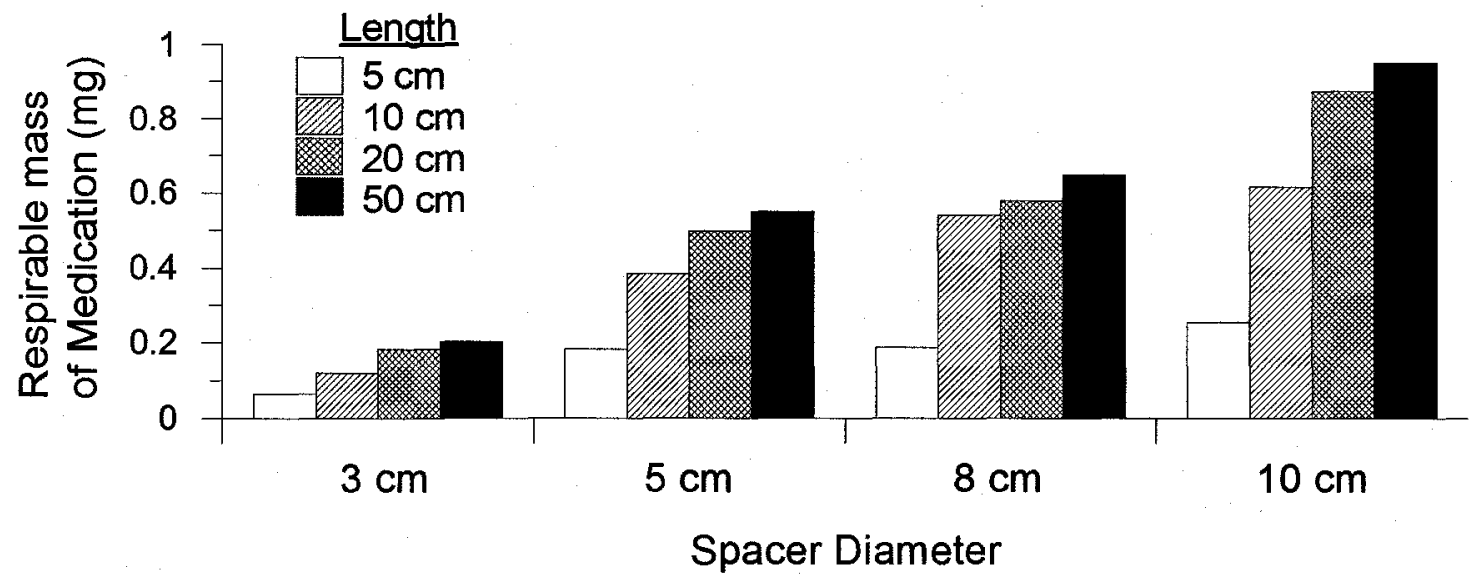

Figure 2.9 - Effect of spacer length and diameter on available respirable mass of medication. Adapted from Barry and O'Callaghan (1995a).

As with pMDIs alone, however, many factors must be taken into consideration when determining the efficacy of an add-on device, including portability. How many asthma-sufferers would be willing to carry a $50 \mathrm{~cm}$ long add-on device everywhere they go? Other factors studied by Barry and O'Callaghan (1995b) include the effects of patient coordination, electrostatic charge on the add-on device as well as multiple actuations of the pMDI on overall efficacy of add-on devices. They concluded that delaying inhalation decreased efficacy, as did multiple actuations, while stating that a low-static device may improve efficacy.

Many different factors affect the ability of an add-on device to improve drug delivery, only a few of which have been mentioned here. Realistically, a comprehensive and systematic study and optimization of spacer devices can only be carried out using numerical techniques, and reliable numerical simulations require well-characterized inlet conditions. 


\section{Chapter 3: Experimental Setup and Procedure}

\subsection{Objectives}

The main objective in the design and setup of the experimental apparatus described here was to make repeatable measurements of the spray issued from a pMDI. To achieve this objective, the pMDI had to be installed in a fixed position, where it could be actuated in a repeatable fashion. Cameras and a laser also had to be installed, aligned and calibrated in such a way as to allow accurate measurement and to reduce uncertainty.

\section{2 pMDI Apparatus}

A pneumatic cylinder was used to actuate the pMDI as shown in Figure 3.1. This ensured that the pMDI was actuated with consistent speed and force. The cylinder was actuated by air at a pressure of $30 \mathrm{psig}$, providing $27 \mathrm{lbf}$. The air was directed into the cylinder by a solenoid valve. Power to the solenoid valve was controlled via a mechanical relay, which in turn was controlled by a timed voltage output from a computer running simple control software written in LabVIEW (National Instruments). This allowed the apparatus to be triggered multiple times without user interaction. To ensure that the pMDI would not move during the triggering process or between trials, the pMDI mount as well as both camera mounts were bolted to a 36 " by 12 " optical 
breadboard which in turn was bolted to a $72 "$ by $36 "$ by $10^{\prime \prime}$ thick, marble work table. As shown in Figure 3.2, the pMDI mount consisted of a negative moulded replica of the pMDI casing manufactured in-house using Bondo Automotive Body Filler (Bondo Corporation, Atlanta, GA, USA). This mould was then glued to a 1" 1 1" steel box beam. One end of the box beam was cut at an angle of $15^{\circ} \pm 1^{\circ}$ and welded to a $1 / 4^{\prime \prime}$ steel plate. The steel plate was then bolted to the optical table.

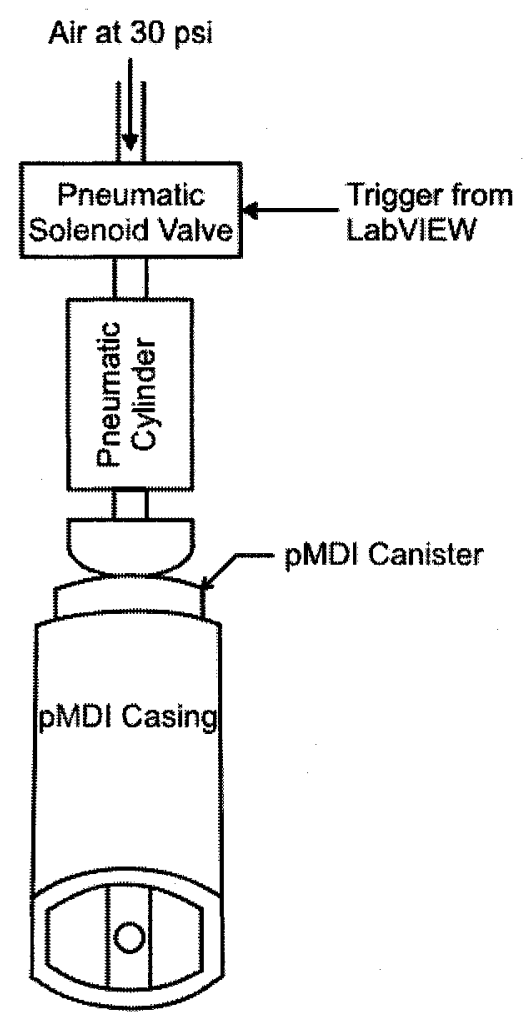

Figure 3.1 - Mechanism to actuate the pMDI. 


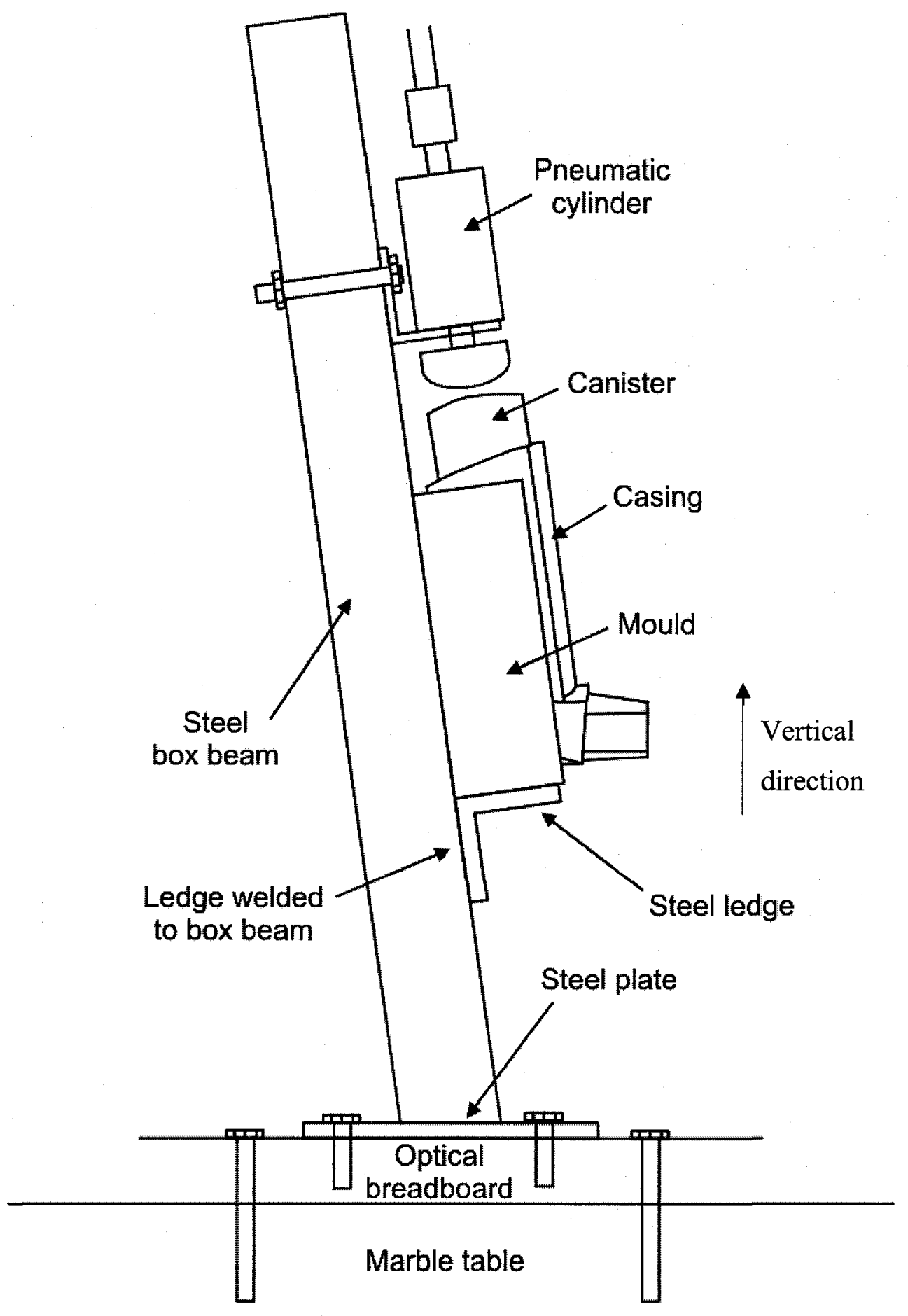

Figure 3.2 - pMDI mounting apparatus. 


\subsection{Air Flow Apparatus}

In some measurement cases an air flow was forced through the pMDI during actuation to simulate an added inhalation flow component, as shown in Figure 3.3. A mass-flow controller (Teledyne Hastings Instruments, Hampton, Virginia, USA) with a maximum flow rate of 100 SLPM was used to force 30 SLPM of air through the pMDI casing and out of the mouthpiece. Air flow rates used to simulate steady breathing in the past include $15,25,28.3,30,35,60,90$, and 120 litres per minute (Han et al., 2002; Cheng, 2003; Heenan et al., 2003; Johnstone et al., 2004; Heenan et al., 2004; Isaacs et al., 2006; Jin et al., 2007). A flow rate of 30 SLPM was chosen so as to allow comparison with as many previous works as possible.

To measure the flow of the air alone, as well as to improve PIV measurements when the spray was present, this air flow was first seeded with olive oil droplets. This was accomplished using two chambers. In the first, the flow enters via a Laskin nozzle submerged in oil which creates a polydisperse distribution of particles with diameters of the order of $1 \mu \mathrm{m}$ (Fischer et al., 1994; Willert et al., 1996). The second chamber contains an inclined plate at its inlet. Any coalescing droplets larger than approximately $4-5 \mu \mathrm{m}$ that are unable to properly track the air flow impact this plate. Echols and Young (1963) as well as Meyers (1991) describe this method for generating the droplets. Based on Melling (1997), Appendix A.8 shows the ability of droplets generated in this fashion to track the secondary air flow.

The flow then enters the pMDI casing via a plastic tee. The pneumatic cylinder enters via the second branch of the tee, while the pMDI is inserted into the third. This is 
shown in Figure 3.3. To prevent leakage, the pneumatic cylinder is inserted into an aluminum bushing slightly smaller than the tee opening. An o-ring is fitted to the bushing and coated with vacuum grease. A special collar was designed for the pMDI casing and manufactured using a rapid-prototyping machine (The Dimension 3D Printing Group, Eden Prairie, Minnesota, USA) to ensure a good seal between the pMDI casing and the tee. The front of the collar was painted black to reduce reflection of the laser. The collar is shown, both alone and fitted to the casing in Figure 3.4, and attached to the pMDI mount in Figure 3.5.

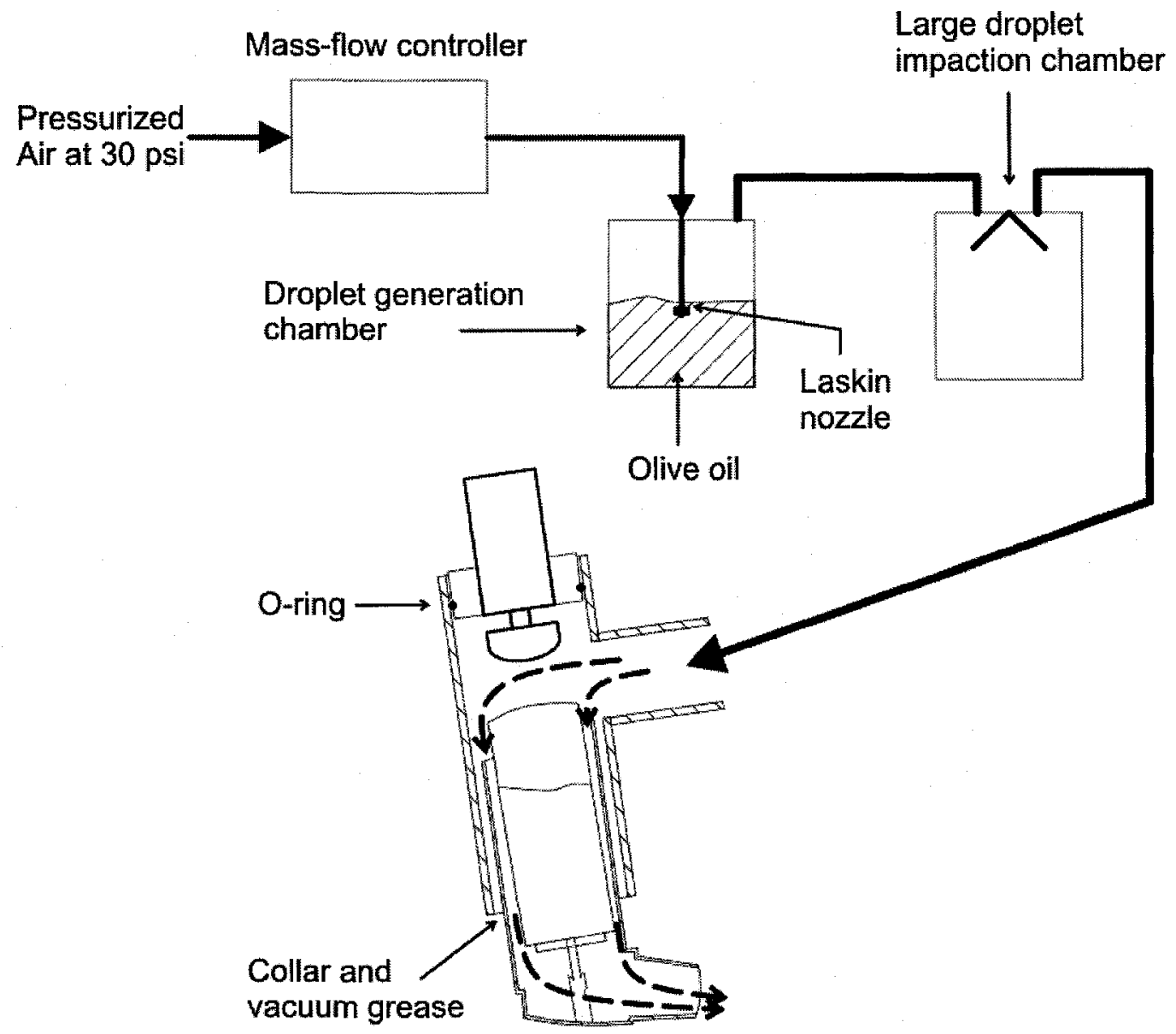

Figure 3.3 - Schematic of the apparatus used to force an air flow, seeded with olive oil particles, through the pMDI casing. 

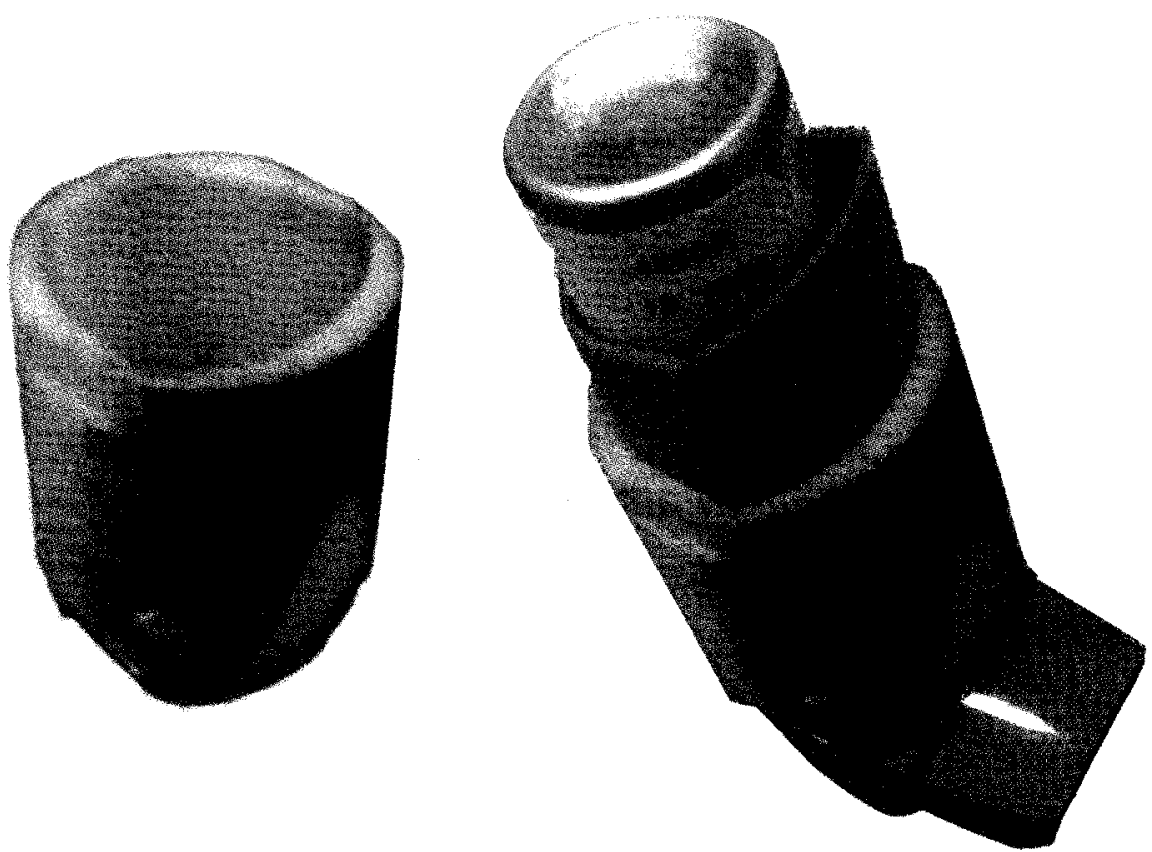

Figure 3.4 - Photograph of the collar used to seal the gap between the pMDI casing and the tee.

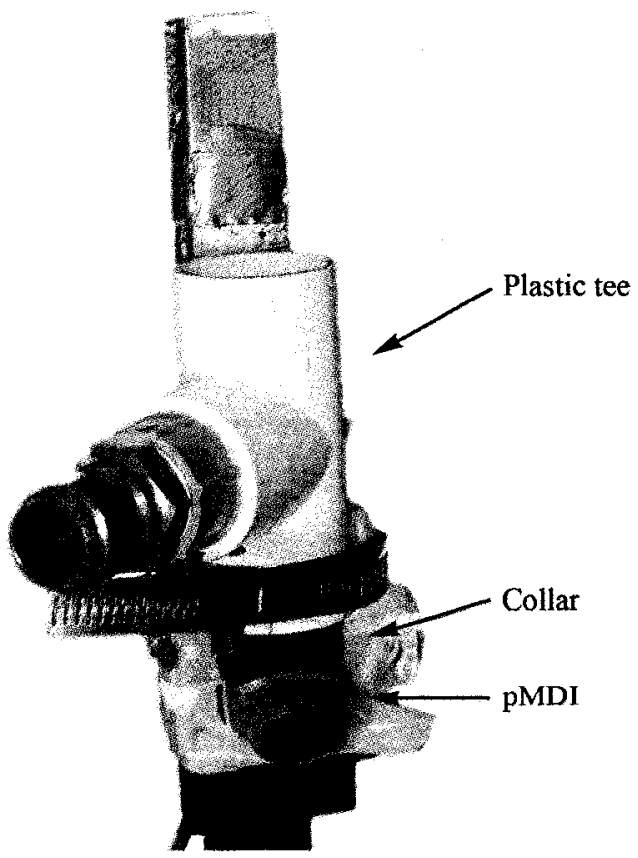

Figure 3.5 - Photograph of a pMDI inserted into the plastic tee via the collar. 


\subsection{Particle Image Velocimetry (PIV) System}

PIV is a non-intrusive optical measurement technique that measures the instantaneous two-dimensional vector velocity field throughout a plane. This is achieved by taking two pictures of the flow and performing a statistical cross-correlation between them. Typically, flows need to be seeded with visible particles which are assumed to track the flow more or less perfectly. In the case of pMDIs, the drug and propellant particles themselves represent the flow being measured, so except for measurements in an added co-flow stream, no seeding is required.

The general process involved in measuring velocities using PIV is as follows:

1. The flow is seeded with appropriate particles.

2. The flow is illuminated twice in rapid succession by a laser sheet.

3. The illuminated flow field is captured as two separate images by a camera.

4. The two images are analyzed using statistical cross-correlation to determine the velocity at a number of discrete locations in the image.

Because the spray issued from a pMDI already contains solid and liquid particles, the first step is not necessary when measuring the spray itself.

\subsubsection{Illumination}

The image plane needs to be illuminated to provide sufficient image contrast to distinguish a particle from background noise. The requirement that the exposure time be short so as to "freeze" the motion of a particle necessitates a powerful light source. Thus a Nd:YAG laser with a maximum fluence of $120 \mathrm{~mJ}$ per pulse and frequency doubled to a 
wavelength of $532 \mathrm{~nm}$ was used (Solo PIV 120, New Wave Research, Fremont, California, USA), allowing the particles to be sufficiently illuminated during the laser pulse time of about 5 ns.

To form the laser beam into a suitable size sheet, a plano-concave lens with a focal length of $38 \mathrm{~mm}$ was placed $5 \mathrm{~cm}$ in front of the laser beam. This was followed by a $100 \mathrm{~mm}$ diameter, symmetrical convex lens placed with its focal point on the cylindrical lens, $1000 \mathrm{~mm}$ away, as shown in Figure 3.6. This formed a collimated light sheet $100 \mathrm{~mm}$ high. As shown in Figure 3.7, the laser system was positioned so that the laser sheet passed through the measurement region when the sheet was narrowest, a point called the waist. This was necessary so as to illuminate fewer particles in the transverse (z) direction, since initial images taken with a wider laser sheet provided poor correlation due to the high spray density. 


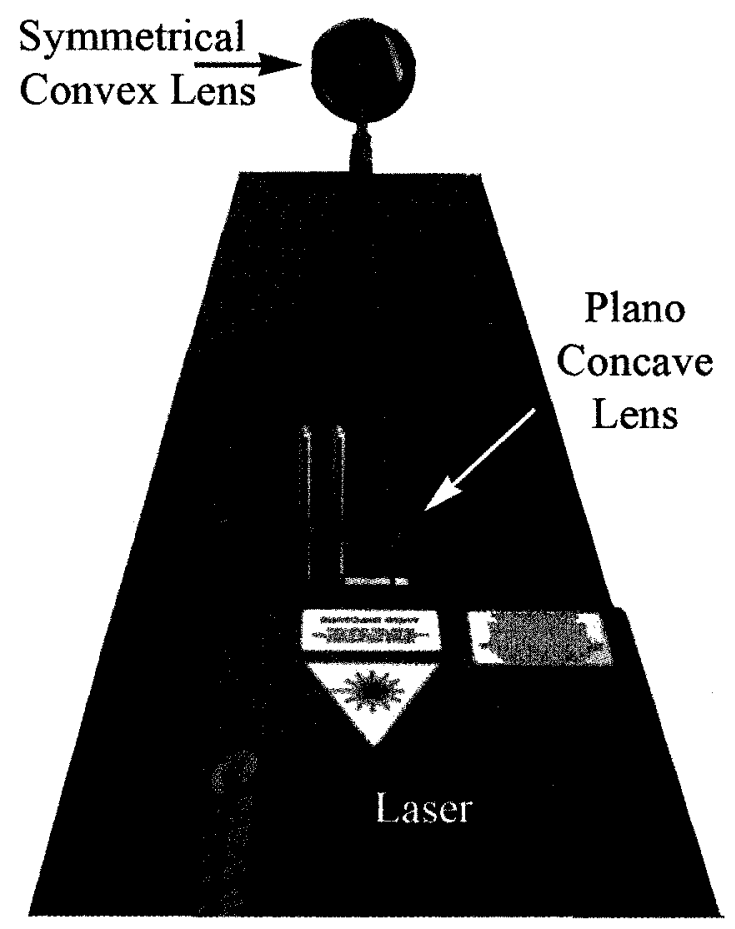

Figure 3.6 - The laser beam passes through a plano-concave lens followed by a symmetrical convex lens, forming a sheet $100 \mathrm{~mm}$ high.

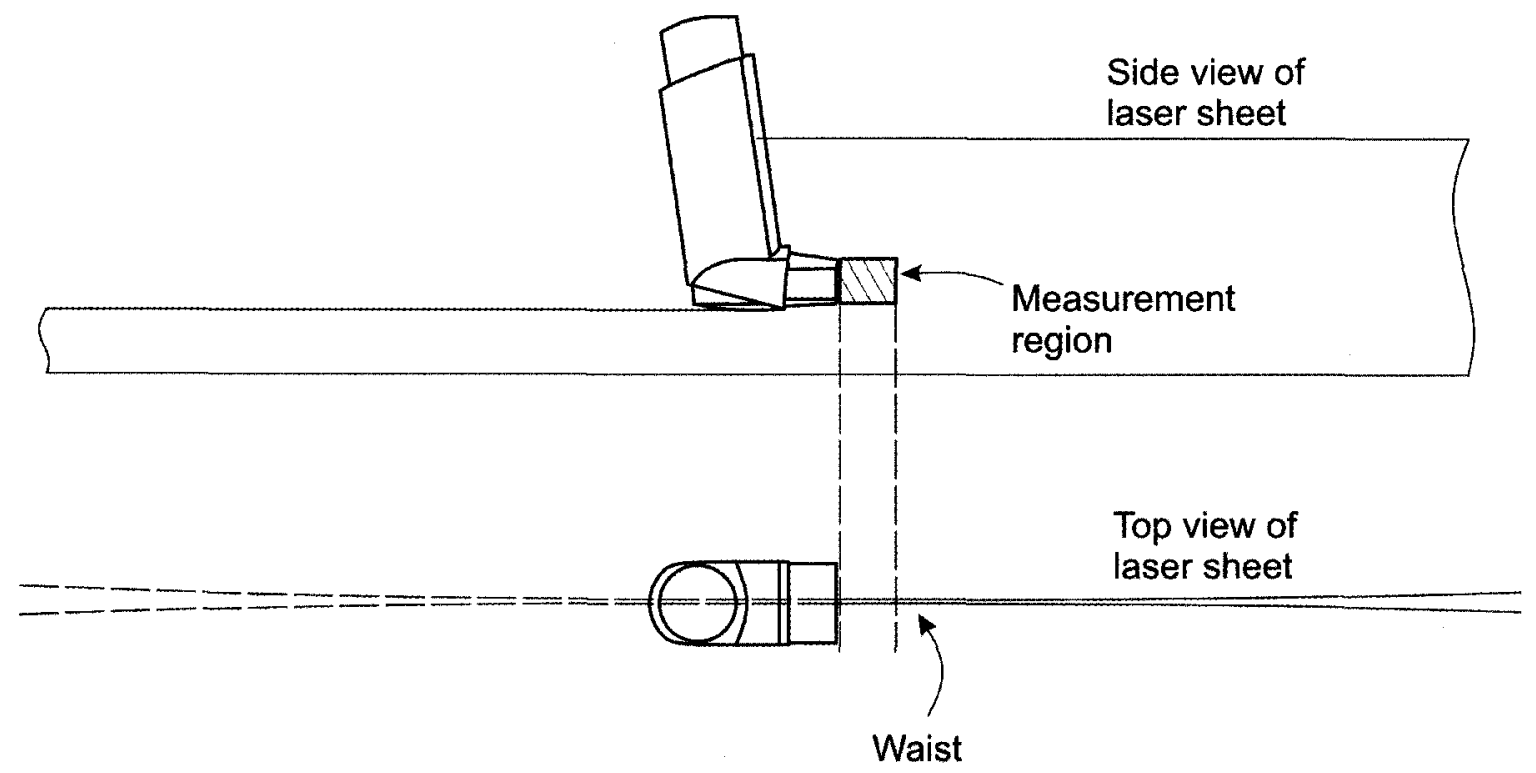

Figure 3.7 - The laser is positioned so that the measurement region is at the waist (the narrowest point in the laser sheet). 
A key experimental parameter when using particle image velocimetry is the interframe time, $\Delta t$. This is the time between laser pulses, which is also the effective time delay between the first and second images. The interframe time must be a compromise between three factors affecting the nature and accuracy of the final data, as presented below in Table 3.1. The approximate interframe time was initially estimated to be $\sim 75 \mathrm{~ms}$. While taking test images of the pMDI flow and examining the resulting images and resultant flow fields, it was determined that a much shorter interframe time was needed. This was due to the high optical density of the spray and strong velocity gradients present in pMDI flow. A final interframe time of $1.2 \mu$ s was used, allowing most particles to move between five and 15 pixels. For measurements of the much slower moving co-flow of air only, an interframe time of $50 \mu$ s was found to be optimal.

Table 3.1 - Factors affecting the choice of PIV interframe time

Decreasing interframe time Increasing interframe time

Approaching true instantaneous velocity

Decreased displacement of a particle increases

the relative uncertainty of the displacement

Captures strong velocity gradients
Average velocity between $t_{0}$ and $t_{1}$ Increased displacement of a particle decreases the relative uncertainty of the displacement Strong velocity gradients cause lost data

\subsubsection{Camera and Lens}

A progressive scan interline transfer CCD camera (Imager Pro 2M, LaVision GmbH, Gottingen, Germany) specially designed for PIV was used to collect images for all PIV measurements. The camera uses frame straddling, allowing it to record two separate exposures less than $500 \mathrm{~ns}$ apart, temporarily storing the first image in masked-off 
portions of the CCD. The image resolution of the CCD is $1600 \times 1200$ square picture elements (pixels) each measuring $7.4 \mu \mathrm{m}$ across. The intensity resolution of the analog to digital conversion is 12 bits. The maximum framerate is 30 frames per second or 15 PIV image pairs per second.

The camera was used with a Micro-Nikkor lens with a fixed focal length of $105 \mathrm{~mm}$ and a maximum aperture of $\mathrm{f} / 2.8$. A $10 \mathrm{~nm}$ bandpass filter, centred at a wavelength of $532 \mathrm{~nm}$, was affixed to the lens. This restricted the amount of ambient light entering the lens, making the effective exposure time equal to the pulse duration of the laser ( $3-5 \mathrm{~ns})$, as indicated in the timing diagram shown in Figure 3.8.

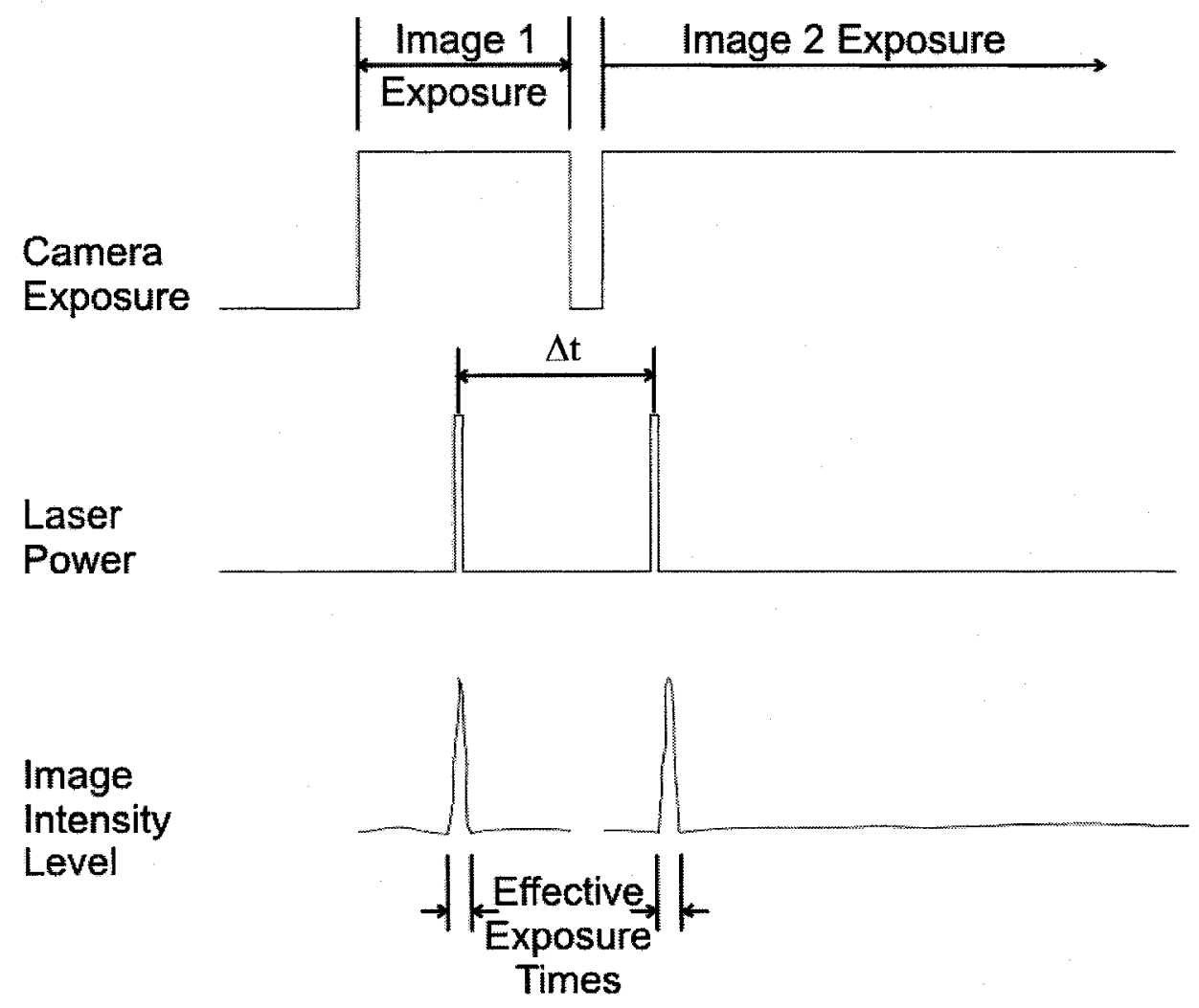

Figure 3.8 - Timing diagram showing the effective exposure time of the PIV camera system. 


\subsubsection{Alignment and Camera Calibration}

The image plane is restricted to the plane illuminated by the laser. For this reason, the laser sheet must be aligned with the desired measurement plane, and the camera must be aligned with, and focused on, the laser sheet.

To accomplish this, a line was etched along the central vertical plane of the pMDI using a surface plate and a height gage. The pMDI was then mounted as described in Section 3.2 and levelled to ensure that it was as close to vertical as possible. The laser was then fired at the pMDI. The laser mount includes a three-way translation stage, which permitted the sheet to be aligned with the etched line on the pMDI, and the location of the laser beam waist to be positioned precisely relative to the pMDI exit plane. The laser mount was also levelled using adjustable feet to ensure rotational alignment of the laser sheet with the scratched line.

Once the laser was aligned with the pMDI, a calibration plate was needed to apply a scale and to correct for distortion in the camera images. A calibration plate provided by the software developer (LaVision GmbH, Gottingen, Germany) consisting of an array of circles $1.2 \mathrm{~mm}$ in diameter spaced $5 \mathrm{~mm}$ apart was placed in the desired measurement region. A picture of the calibration plate, as seen by the camera used for the PIV images, is shown in Figure 3.9. This calibration plate was mounted on two linear stages and a three-axis-of-rotation tripod head (410 Junior Geared Head, Gruppo Manfrotto Srl, Italy) to permit translational and rotational alignment. A block of aluminum was machined to the appropriate thickness so as to ensure that the calibration plate was placed at the same height as the pMDI. The calibration plate was then aligned with the laser sheet, and thus 
aligned with the centerline of the pMDI. The complete calibration plate mounting apparatus is shown in Figure 3.10.

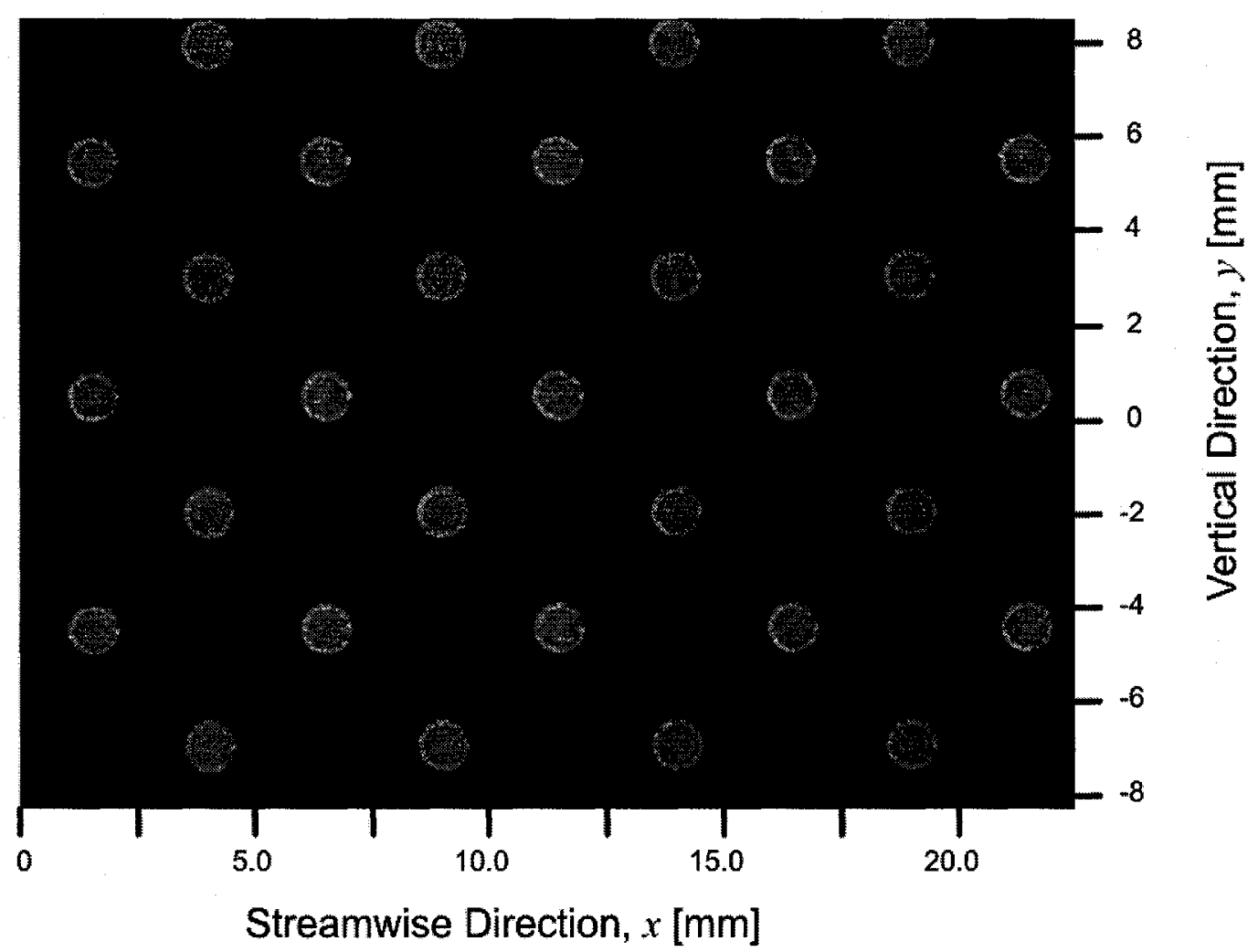

Figure 3.9 - The image of the calibration plate used to calibrate the Imager Pro 2M. 


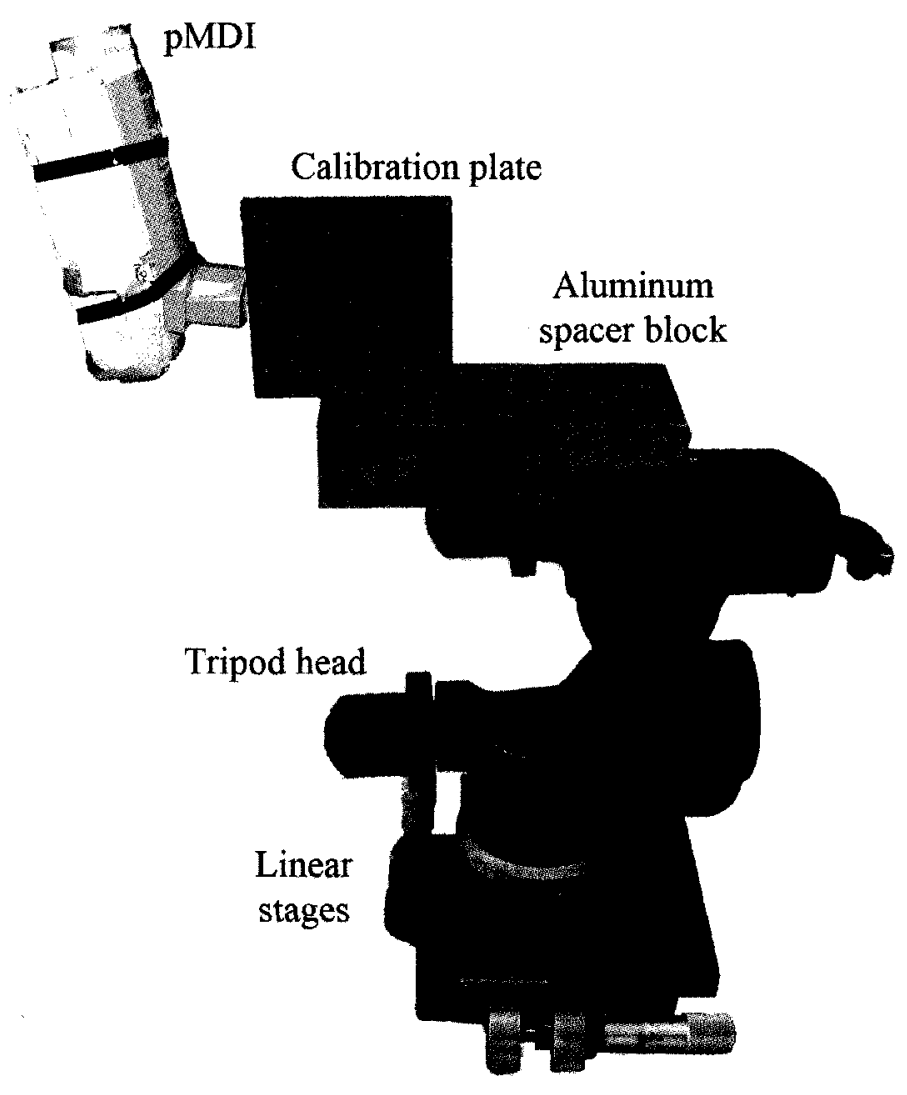

Figure 3.10 - The calibration plate mounting apparatus.

The camera was also placed on two linear stages for translational alignment and a three-axis-of-rotation tripod head for rotational alignment. A rail carrier was used for coarse adjustment of the distance from the camera to the image plane. A block of aluminum was machined to an appropriate thickness to act as a spacer, ensuring the camera was centred vertically in the measurement region. The camera mounting apparatus is shown in Figure 3.11. The measurement regions used when taking images for PIV measurements are shown in Figure 3.12. 


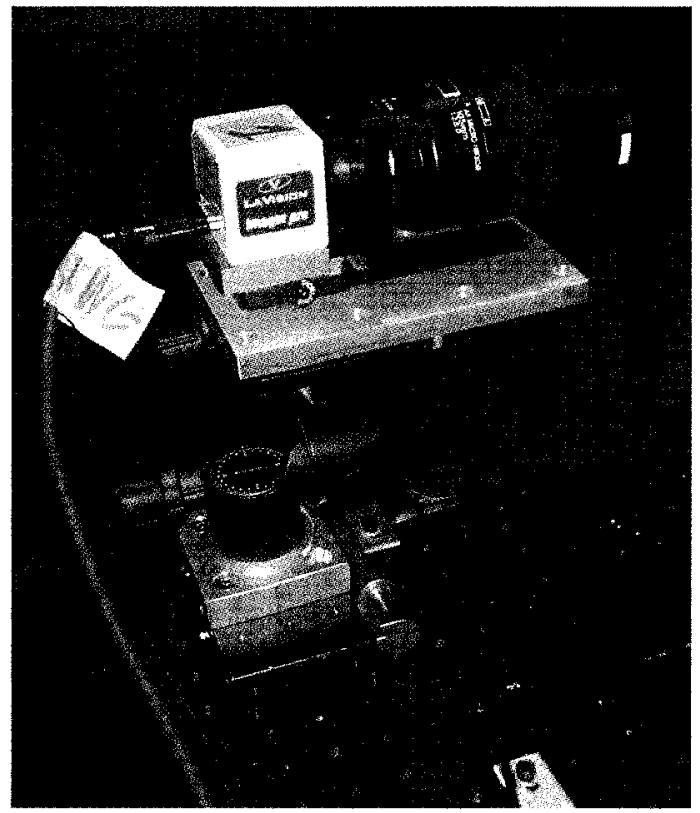

Figure 3.11 - The Imager Pro 2M (PIV camera) mounting apparatus.

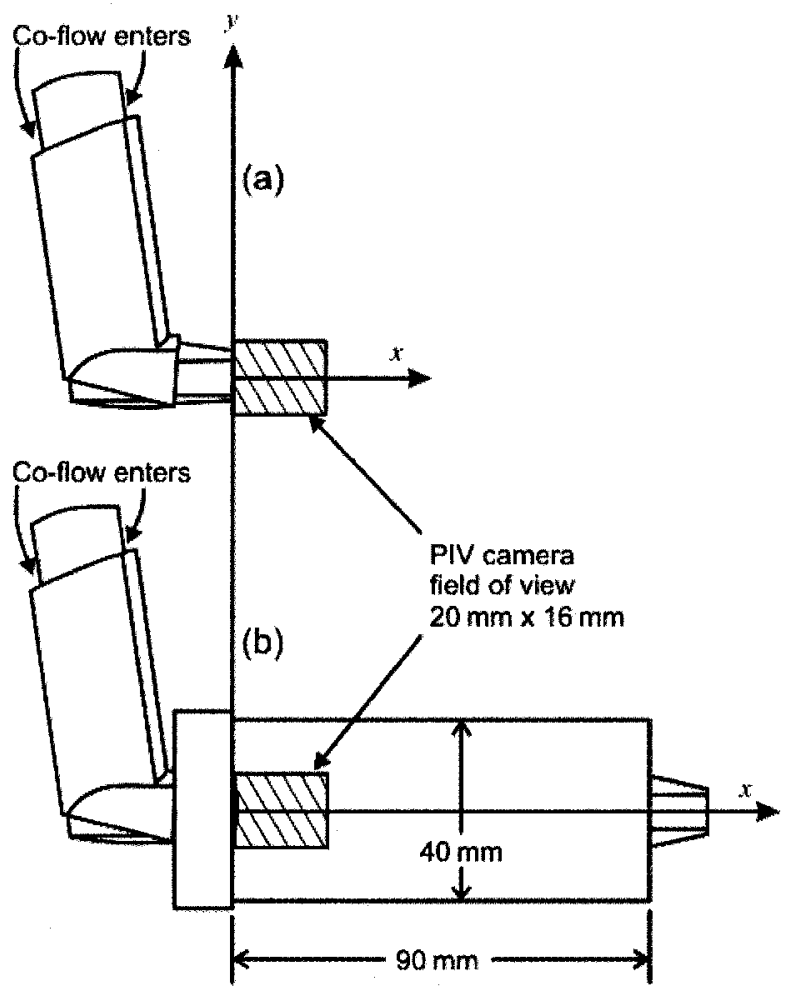

Figure 3.12 - The fields of view for pMDI mouthpiece outlet measurement cases, (a) without and (b) with and add-on spacer device. 
Once the camera was approximately aligned with the measurement region, it was turned on and aligned in real-time by examining the image of the calibration plate. Because the calibration plate was only partially in the laser path, the laser could not be used to illuminate the calibration plate during calibration because light reflected directly into the $\mathrm{CCD}$ can damage the camera. To ensure that adequate illumination reached the CCD through the bandpass filter, a desk lamp with a 100-Watt light bulb was placed very close (less than $10 \mathrm{~cm}$ ) to the calibration plate. This provided enough light in the filter bandpass range to illuminate the image during the maximum allowable camera exposure time of $66 \mathrm{~ms}$. Focusing of the camera lens was first performed at its lowest $\mathrm{f}$-number of 1.4 , where depth of field is smallest, to ensure the calibration plate was centred in the final, expanded depth of field.

Once a sufficiently bright image was visible, the tripod head and linear stages were used to align the camera field of view with the dots on the calibration plate. The rotational controls on the tripod head were first used to adjust the field of view until the array of dots appeared equidistant and parallel to the edges of the field of view. To maximize the field of view and reduce reflections from the pMDI, the camera was then translated so that less than a millimetre of the pMDI mouthpiece was visible. This image (previously shown in Figure 3.9) was then used by the FlowMaster PIV software (LaVision GmbH, Gottingen, Germany),software to apply a physical length scale to the images as well as to correct for distortion due to the camera lens. Once the software has determined the location of the images of the dots, it attempts to model the transformation from the image coordinates (in pixels) to the real-world using either one of two models. 
The simplest is a pinhole camera model, and is useful when the calibration plate does not cover the entire field of view. If the calibration plate does cover the entire field of view, a third-order polynomial model usually provides a better transformation. This modeling, rather than the application of a pixel-by-pixel transformation, greatly reduces the computational time required to perform the PIV calculations. The root-mean square (rms) difference between the applied model and a true pixel-by-pixel transformation is then calculated. Though most of the data presented herein use the third-order polynomial model, the camera-pinhole model was used for the measurements taken inside the add-on device. According to the software manual (LaVision, 2006a, 2006b), "a value lower than 1 pixel is good, a value lower than 0.3 pixel is excellent, values higher than 2 pixel are questionable." The rms fit error for all experimental cases was less than 0.5 , as outlined in Table 3.2, below.

Table 3.2 - The error associated with the model used to transform pixel coordinates to realworld coordinates.

\begin{tabular}{ccc}
\hline Measurement Case & Rms error of fit & Model used \\
\hline Brand Alopen air & 0.22616 & $3^{\text {rd }}$ order polynomial \\
Brand B/open air & 0.22616 & $3^{\text {rd }}$ order polynomial \\
Co-flow of air only & 0.23557 & $3^{\text {rd }}$ order polynomial \\
Brand A/co-flow of air & 0.23557 & $3^{\text {rd }}$ order polynomial \\
Brand A/co-flow/add-on & 0.3919 & Camera-pinhole \\
device & & \\
Brand A/co-flow/exit of & 0.45971 & Camera-pinhole \\
add-on device & & \\
\hline
\end{tabular}




\subsubsection{Triggering the PIV Data Acquisition Process}

To trigger the PIV system in a repeatable fashion, an optical method was adopted. A Helium-Neon (He:Ne) laser was directed through two 1/16" diameter holes in the pMDI casing and into a photodiode on the opposite side of the casing. The location of the holes and the laser position are shown in Figure 3.13. When the pMDI canister was depressed by the pneumatic cylinder, the laser beam was interrupted. A $+5 \mathrm{VDC}$ power source was connected in series with the photodiode and a 74LS04 solid-state inverter, which has a maximum switching delay time of $15 \mathrm{~ns}$. This sent a +5VDC signal to the PIV computer, which then commenced the rest of the data acquisition process.
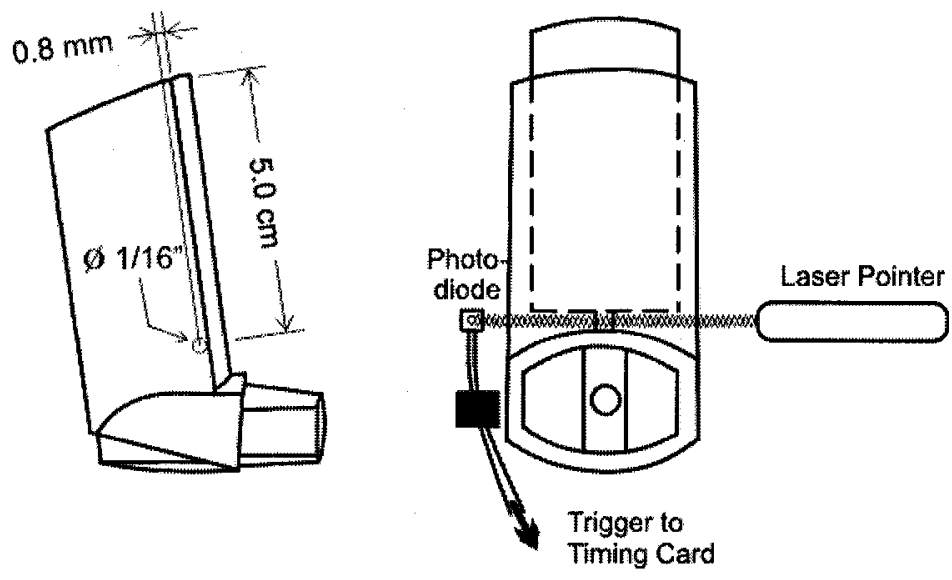

Figure 3.13 - The location of the hole where the He:Ne laser passes to trigger the data acquisition system.

In order to ensure that this triggering process remained consistent throughout all measurements, the same pMDI casing, and thus the same hole location, was used for every canister and every case. To prevent the buildup of medication in the nozzle and mouthpiece, the casing was washed after every 200 actuations, as follows: 
- Immerse casing in isopropyl alcohol for 60 seconds

- Force isopropyl alcohol through nozzle with squeeze bottle

- Rinse casing with water

- Verify nozzle performance: for a given flow of water through the nozzle the stream of water emitted by the nozzle must travel a distance equal to that produced by a new pMDI casing under the same conditions

- Dry with pressurised air

- Cover trigger holes with transparent tape to prevent leakage of inhalatory co-flow

\subsection{Spray Imaging System}

A second camera was used to obtain images of the spray over a larger FOV. The camera used for this purpose was the Imager Intense (LaVision $\mathrm{GmbH}$, Gottingen, Germany). Similar to the Imager Pro $2 \mathrm{M}$, the Imager Intense contains a progressive scan interline transfer CCD allowing it to take two images in quick succession for PIV measurements if needed. However, in this case it was used in single frame mode. The Imager Intense camera has an image resolution of $1376 \times 1040$ square pixels each measuring $6.45 \mu \mathrm{m}$ across, and an intensity resolution of 12 bits. The maximum framerate is 20 frames per second. 
The Imager Intense was fitted with a Micro-Nikkor lens with a fixed focal length of $50 \mathrm{~mm}$ and a maximum aperture of $\mathrm{f} / 2.8$. A $10 \mathrm{~nm}$ bandpass filter, centred at a wavelength of $532 \mathrm{~nm}$, was affixed to the lens. This restricted the amount of ambient light entering the lens, making the effective exposure time equal to the pulse duration of the laser (3-5 ns).

The camera was then mounted on two linear stages for $x-z$ translational alignment, and a tripod head for three-axis rotational alignment, both shown in Figure 3.10. A FOV of $78 \times 60 \mathrm{~mm}$ was used. Preliminary results indicated that the spray was sometimes deflected below the horizontal ( $x z)$, so the FOV was slightly offset from the $x$ axis so as to capture more of the event. A calibration was then performed for scale and distortion using the same calibration plate and methods described in Section 3.3.3. Since the calibration plate was not large enough to cover the entire FOV, the third-order polynomial coordinate transformation mapping method could not be used, leaving only the camera pinhole model. This gave an rms fit error of 0.16 pixels.

\subsection{Data Acquisition Sequence}

The entire experimental setup is summarized in Figure 3.14. 

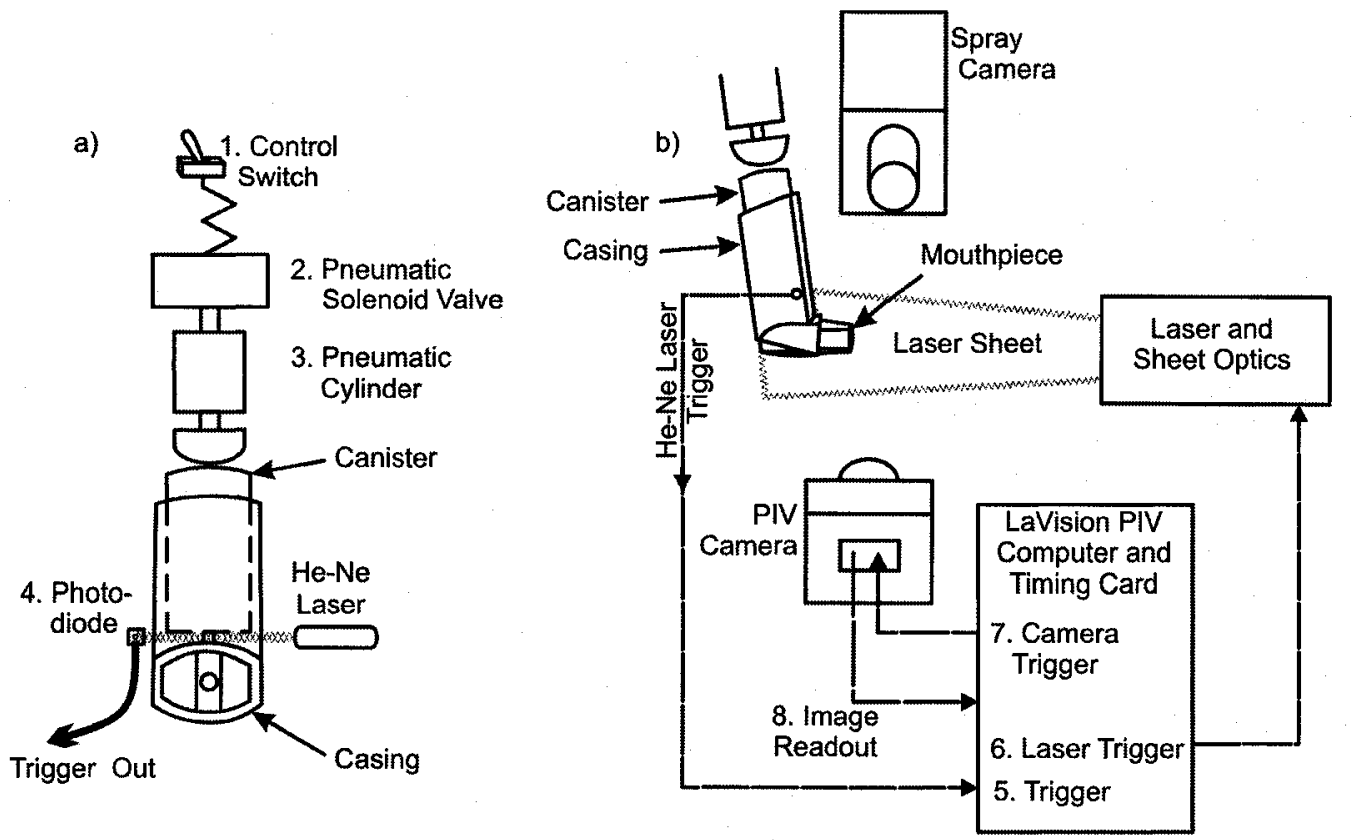

Figure 3.14 - Experimental setup

The sequence of events leading to the acquisition of a single set of two PIV images and one spray image is as follows:

- Figure 3.14(a)

1. A control switch is toggled, delivering power to the pneumatic solenoid valve

2. The pneumatic solenoid valve toggles, delivering air at $30 \pm 0.5 \mathrm{psi}$ to the pneumatic cylinder

3. The pneumatic cylinder extends, placing a force of $27 \pm 5$ pounds on the pMDI canister

4. The pMDI canister is depressed, blocking the He-Ne laser beam. The photodiode sends a +5VDC signal to the PIV computer system.

- Figure 3.14(b)

5. The trigger signal is received from the photodiode. 
6. Pairs of $+5 \mathrm{VDC}$ signals are sent to the dual-head laser, causing it to fire each of the two flashlamps and subsequently each of the two Q-switches to release the two, precisely timed laser pulses.

7. A series of $+5 \mathrm{VDC}$ signals are sent to the PIV and spray cameras, triggering image acquisition in precise timing with the laser pulses

8. The images are read from the cameras back into the computer.

The time delay after actuation is thus defined as the time interval between the breaking of the He:Ne laser beam by the canister being compressed and the time of the PIV laser pulses.

\section{7 pMDI Procedures}

Throughout the experimental setup and measurement process, many steps were taken in an attempt to reduce systematic bias. Ventolin HFA (GlaxoSmithKline Inc., Montréal, Québec, Canada) and ratio-Salbutamol HFA (ratiopharm inc., Montréal, Québec, Canada) pMDIs containing salbutamol sulphate were obtained from a local pharmacy. Before measurement, the canisters were primed by first shaking the device and then releasing three sprays without measurement. The instructions included with a pMDI instruct the patient to shake the device before every usage. Time constraints and the experimental setup made shaking between every actuation impractical. To determine how often the canister required shaking, an initial set of 150 measurements were made at a single time delay, with the canister being shaken ten times after every $25^{\text {th }}$ actuation. The average velocity in various regions of the flow was then plotted as a function of " $n^{\text {th }}$ actuation since shaking." A sample plot is shown in Figure 3.15, where the general 
downward trend observed indicates that more frequent shaking than every 25 actuations is required.

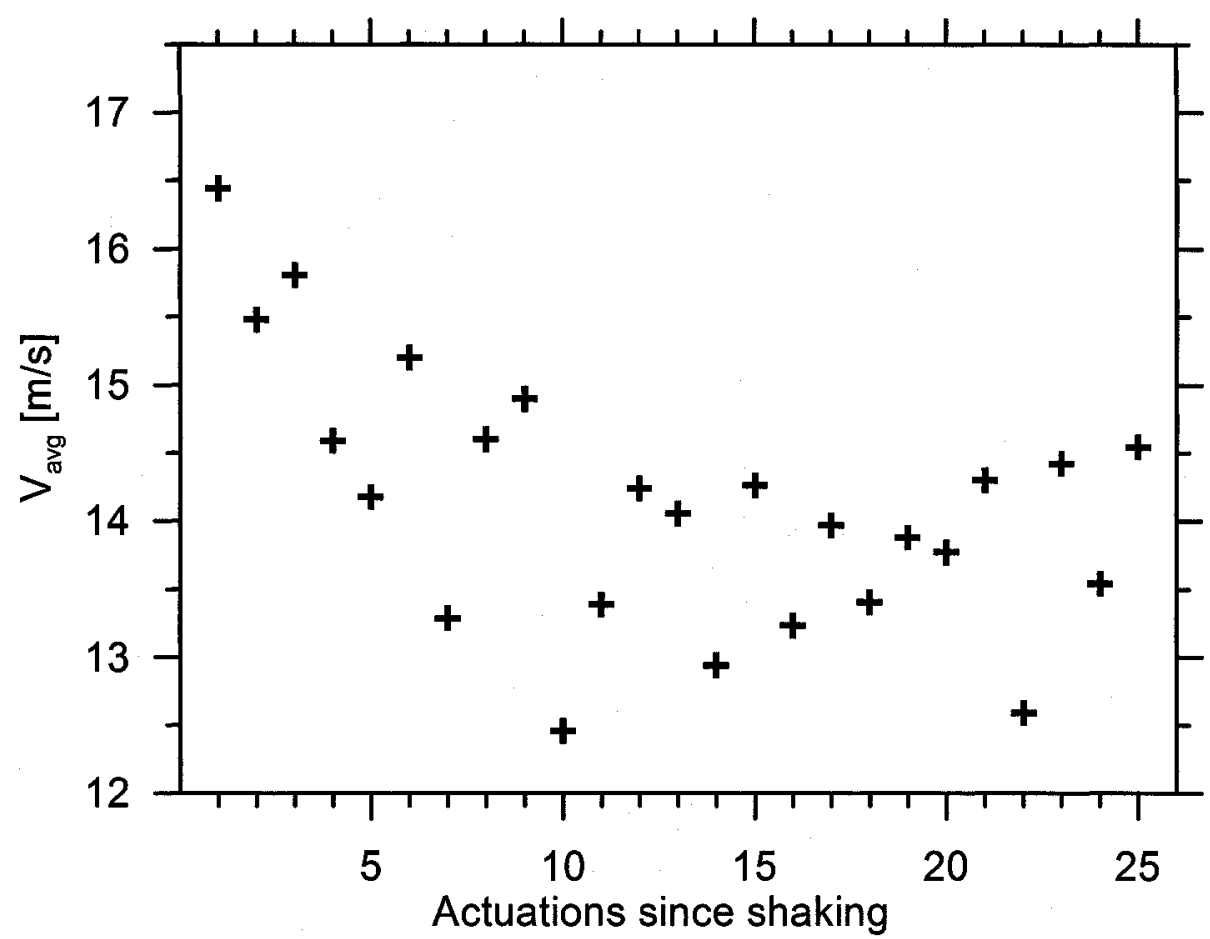

Figure 3.15 - Average velocity along spray centreline for the first 25 actuations following canister shaking. ratio-Salbutamol HFA, $10 \mathrm{~ms}$.

A shaking frequency of every ten actuations was then investigated. One hundred measurements were made in total allowing 10 samples per "nth actuation since shaking". All measurements were made at a single time delay of $10 \mathrm{~ms}$. Figure 3.16 shows the average, minimum and maximum velocity along the line $y=0 \mathrm{~mm}$ when the canister was shaken after every 10th actuation. Uncertainty bars depict the standard devation. 


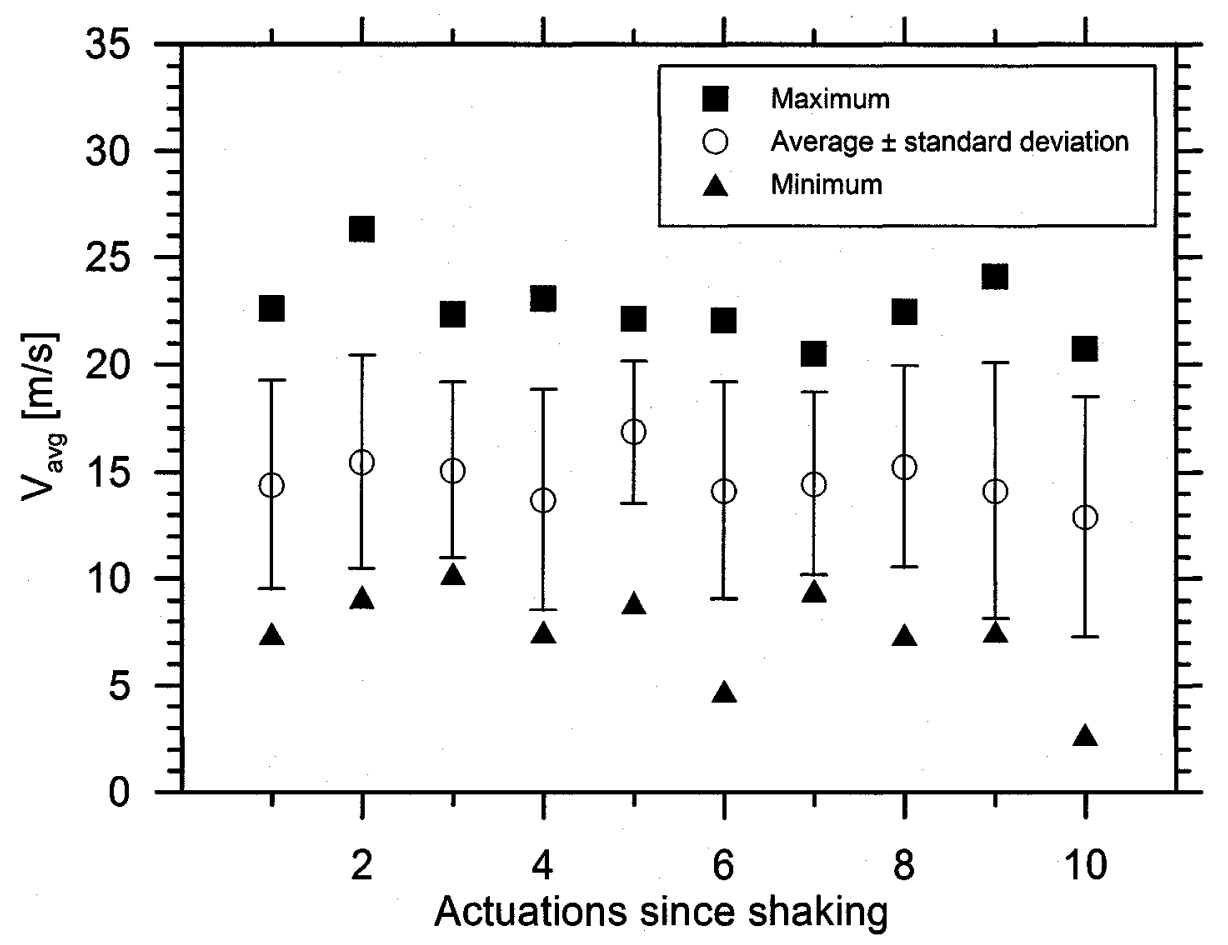

Figure 3.16 - Minimum and maximum velocity, as well as average velocity and its standard deviation, along the spray centreline for the first $\mathbf{1 0}$ actuations following canister shaking. ratio-Salbutamol HFA, 10 ms.

Spray-to-spray variability is significant and there is no clear trend in averaged velocity with number of actuations after shaking. Student's t-test for independent samples was applied to determine if there existed a statistically significant difference between the velocities of the first actuation after shaking and the velocities of each subsequent actuation. The magnitude of the velocity at $(2.5,0) \mathrm{mm}$ was extracted from the raw vector velocity flow fields, and used to determine the mean and standard deviation of the magnitude of velocity at this point. Student's t-test calculates a t-score as follows: 


$$
t=\frac{\left|\bar{x}_{1}-\bar{x}_{2}\right|}{\sqrt{\left(\frac{\left(n_{1}+n_{2}\right)}{n_{1} n_{2}}\right)\left(\frac{\left(n_{1}-1\right) s_{1}^{2}+\left(n_{2}-1\right) s_{2}^{2}}{n_{1}+n_{2}-2}\right)}}
$$

where $\bar{x}_{1}$ and $\bar{x}_{2}$ are the mean velocities for each set, $n_{1}$ and $n_{2}$ are the number of samples in each set, and $s_{1}$ and $s_{2}$ are the standard deviations within each set. This score is an intermediate statistic that correlates to a probability that the two means represent significantly different samples. Using Student's probability table for small sample sizes, a two-tailed distribution having 20 samples in two sets of 10 (i.e. 18 degrees of freedom), a t-score of 2.101 or greater represents a 95 percent or greater probability that the two samples are different.

Using this criterion to compare the first actuation after shaking with the nine subsequent actuations, Table 3.4 shows that there is always a less than 95 percent probability $(\mathrm{p}<0.05)$ that a subsequent actuation is different from the first actuation. Also, there seems to be no particular trend in the standard deviation or the t-score, as might be expected if subsequent actuations deviated more and more from the original (freshly-shaken) actuation. 
Table 3.4 - Statistical Significance Analysis of Shaking Frequency. ratio-salbutamol HFA. $x=2.5 \mathrm{~mm}, y=0 \mathrm{~mm} .10 \mathrm{~ms}$.

\begin{tabular}{ccccc}
\hline Data Set & $\begin{array}{c}\text { Mean } \\
(\mathrm{m} / \mathrm{s})\end{array}$ & $\begin{array}{c}\text { Std. Dev. } \\
(\mathrm{m} / \mathrm{s})\end{array}$ & $\begin{array}{c}\text { t-score } \\
\text { relative to } \\
\text { Shake }+1\end{array}$ & $\begin{array}{c}\text { Significant? } \\
(\mathbf{p}<\mathbf{0 . 0 5}) \\
(\mathbf{t}>\mathbf{2 . 1 0 1})\end{array}$ \\
\hline Shake +1 & 30.9 & 5.56 & & NO \\
Shake +2 & 31.1 & 8.93 & 0.04 & NO \\
Shake +3 & 33.1 & 7.17 & 0.77 & NO \\
Shake +4 & 34.8 & 5.09 & 1.63 & NO \\
Shake +5 & 30.8 & 6.81 & 0.04 & NO \\
Shake +6 & 30.9 & 4.57 & 0.01 & NO \\
Shake +7 & 30.9 & 3.03 & 0.01 & NO \\
Shake +8 & 30.6 & 5.27 & 0.13 & NO \\
Shake +9 & 30.9 & 4.62 & 0.01 & NO \\
\hline Shake +10 & 33.9 & 6.97 & 1.04 & \\
\hline
\end{tabular}

Another initial study was performed using pMDI canisters from different manufacturing lots. The results from these tests, shown in Table 3.5, indicated that the canisters did not create significantly different velocities at 12 of 16 times and locations tested. This small difference is considered statistically insignificant, and so all future measurements were made without regard to the canister lot number. 
Table 3.5 - Statistical significance of canister lot number on spray velocity at four locations and four time delays.

\begin{tabular}{|c|c|c|c|c|c|c|}
\hline \multicolumn{7}{|c|}{ At $(x, y)=(2.5 \mathrm{~mm}, 0 \mathrm{~mm})$} \\
\hline $\begin{array}{l}\text { Time } \\
\text { ms }\end{array}$ & $\begin{array}{c}\bar{u} \operatorname{Lot} A \\
\mathrm{~m} / \mathrm{s}\end{array}$ & $\begin{array}{c}\bar{u} \text { Lot } B \\
\mathrm{~m} / \mathrm{s}\end{array}$ & $\begin{array}{c}\text { Percent } \\
\text { Difference }\end{array}$ & $N$ & t-score & $\begin{array}{c}\text { Significant? } \\
(p<0.05) \\
(t>2.021)\end{array}$ \\
\hline 5 & 23.02 & 28.57 & $21.50 \%$ & 33 & 2.03 & YES \\
\hline 10 & 20.93 & 28.20 & $29.61 \%$ & 34 & 3.85 & YES \\
\hline 20 & 28.66 & 24.54 & $15.48 \%$ & 36 & 1.47 & NO \\
\hline 40 & 31.93 & 25.39 & $22.84 \%$ & 36 & 3.48 & YES \\
\hline \multicolumn{7}{|c|}{ At $(x, y)=(7.5 \mathrm{~mm}, 0 \mathrm{~mm})$} \\
\hline $\begin{array}{l}\text { Time } \\
\text { ms }\end{array}$ & $\begin{array}{c}\bar{u} \operatorname{Lot} A \\
\mathrm{~m} / \mathrm{s}\end{array}$ & $\begin{array}{l}\bar{u} \text { Lot } B \\
\mathrm{~m} / \mathrm{s}\end{array}$ & $\begin{array}{c}\text { Percent } \\
\text { Difference* }\end{array}$ & $N$ & t-score & $\begin{array}{c}\text { Significant? } \\
(p<0.05) \\
(t>2.021)\end{array}$ \\
\hline 5 & 23.43 & 26.31 & $11.57 \%$ & 38 & 1.15 & NO \\
\hline 10 & 21.56 & 21.55 & $0.05 \%$ & 38 & 0.00 & NO \\
\hline 20 & 25.40 & 22.08 & $13.98 \%$ & 37 & 1.45 & NO \\
\hline 40 & 30.58 & 27.06 & $12.21 \%$ & 37 & 1.97 & NO \\
\hline \multicolumn{7}{|c|}{ At $(x, y)=(12.5 \mathrm{~mm}, 0 \mathrm{~mm})$} \\
\hline $\begin{array}{l}\text { Time } \\
\text { ms }\end{array}$ & $\begin{array}{c}\bar{u} \operatorname{Lot} A \\
\mathrm{~m} / \mathrm{s}\end{array}$ & $\begin{array}{c}\bar{u} \text { Lot B } \\
\mathrm{m} / \mathrm{s}\end{array}$ & $\begin{array}{c}\text { Percent } \\
\text { Difference* }\end{array}$ & $N$ & t-score & $\begin{array}{c}\text { Significant? } \\
(p<0.05) \\
(t>2.021)\end{array}$ \\
\hline 5 & 24.37 & 25.25 & $3.55 \%$ & 36 & 0.47 & NO \\
\hline 10 & 20.48 & 19.85 & $3.09 \%$ & 38 & 0.36 & NO \\
\hline 20 & 26.27 & 21.03 & $22.19 \%$ & 38 & 1.93 & NO \\
\hline 40 & 28.08 & 26.62 & $5.36 \%$ & 38 & 0.60 & NO \\
\hline
\end{tabular}




\begin{tabular}{|c|c|c|c|c|c|c|}
\hline \multicolumn{7}{|c|}{ At $(x, y)=(17.5 \mathrm{~mm}, 0 \mathrm{~mm})$} \\
\hline Time & $\bar{u}$ Lot $\mathrm{A}$ & $\bar{u}$ Lot $\mathrm{B}$ & Percent & $\mathrm{N}$ & t-score & SSignificant? \\
\hline & $M / s$ & & Difference* & & & $\begin{array}{c}(p<0.05) \\
(t>2.021)\end{array}$ \\
\hline 5 & 21.52 & 24.56 & $13.21 \%$ & 37 & 1.37 & NO \\
\hline 10 & 21.81 & 20.88 & $4.34 \%$ & 37 & 0.55 & NO \\
\hline 20 & 26.83 & 20.90 & $24.86 \%$ & 37 & 2.56 & YES \\
\hline 40 & 26.91 & 28.55 & $5.90 \%$ & 37 & 0.78 & NO \\
\hline
\end{tabular}

*Percent difference is defined as $(2|\mathrm{~A}-\mathrm{B}|) /(\mathrm{A}+\mathrm{B})$

To minimize any bias that may remain, canister rotation schedules like that shown in Table 3.6 were created. For every set of 1000 measurements, five canisters numbered $1-5$ were used. The rotation schedule is thus designed to ensure that each ensembleaveraged measurement will contain data from all canisters at two different states of fullness.

Table 3.6 - Canister rotation schedule used to minimize bias error.

\begin{tabular}{|c|c|c|c|c|c|c|c|c|c|c|}
\hline \multirow{11}{*}{ 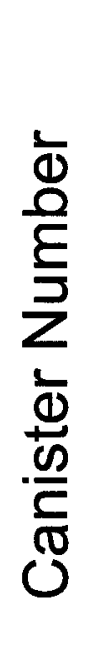 } & 1.3 & 1.7 & 2 & 5 & 10 & 20 & 40 & 60 & 80 & 100 \\
\hline & 4 & 5 & 1 & 2 & 3 & 4 & 5 & 1 & 2 & 3 \\
\hline & 5 & 1 & 2 & 3 & 4 & 5 & 1 & 2 & 3 & 4 \\
\hline & 1 & 2 & 3 & 4 & 5 & 1 & 2 & 3 & 4 & 5 \\
\hline & 2 & 3 & 4 & 5 & 1 & 2 & 3 & 4 & 5 & 1 \\
\hline & 3 & 4 & 5 & 1 & 2 & 3 & 4 & 5 & 1 & 2 \\
\hline & 4 & 5 & 1 & 2 & 3 & 4 & 5 & 1 & 2 & 3 \\
\hline & 5 & 1 & 2 & 3 & 4 & 5 & 1 & 2 & 3 & 4 \\
\hline & 1 & 2 & 3 & 4 & 5 & 1 & 2 & 3 & 4 & 5 \\
\hline & 2 & 3 & 4 & 5 & 1 & 2 & 3 & 4 & 5 & 1 \\
\hline & 3 & 4 & 5 & 1 & 2 & 3 & 4 & 5 & 1 & 2 \\
\hline
\end{tabular}


A number of measurement cases were examined. This includes measuring two different brands of canisters, adding a co-flow of air and adding a commonly used add-on device. The various measurement cases studied are summarized in Table 3.7.

Table 3.7 - Measurement conditions

\begin{tabular}{c|c|c|c|c|c|}
\hline & \multicolumn{1}{|c|}{$\begin{array}{c}\text { ratio- } \\
\text { SALBUTAMOL HFA }\end{array}$} & \multicolumn{5}{|c}{ Ventolin-HFA } \\
\hline $\begin{array}{c}\text { Time delay after } \\
\text { actuation (ms) }\end{array}$ & Quiescent air & $\begin{array}{c}\text { Quiescent } \\
\text { air }\end{array}$ & $\begin{array}{c}\text { With } \\
\text { co-flow }\end{array}$ & $\begin{array}{c}\text { With co-flow and } \\
\text { aerochamber }\end{array}$ & $\begin{array}{c}\text { At aerochamber } \\
\text { outlet }\end{array}$ \\
\hline 0.9 & & $\mathrm{X}$ & & & \\
\hline 1.3 & $\mathrm{X}$ & $\mathrm{X}$ & $\mathrm{X}$ & $\mathrm{X}$ & \\
\hline 1.7 & $\mathrm{X}$ & & $\mathrm{X}$ & $\mathrm{X}$ & \\
\hline 2.0 & $\mathrm{X}$ & $\mathrm{X}$ & $\mathrm{X}$ & $\mathrm{X}$ & \\
\hline 2.5 & & & & & $\mathrm{X}$ \\
\hline 5.0 & $\mathrm{X}$ & $\mathrm{X}$ & $\mathrm{X}$ & $\mathrm{X}$ & $\mathrm{X}$ \\
\hline 7.5 & & & & & $\mathrm{X}$ \\
\hline 10.0 & $\mathrm{X}$ & $\mathrm{X}$ & $\mathrm{X}$ & $\mathrm{X}$ & $\mathrm{X}$ \\
\hline 12.5 & & & & & $\mathrm{X}$ \\
\hline 15 & & & & & $\mathrm{X}$ \\
\hline 20.0 & $\mathrm{X}$ & $\mathrm{X}$ & $\mathrm{X}$ & $\mathrm{X}$ & $\mathrm{X}$ \\
\hline 40.0 & $\mathrm{X}$ & $\mathrm{X}$ & $\mathrm{X}$ & $\mathrm{X}$ & $\mathrm{X}$ \\
\hline 60.0 & $\mathrm{X}$ & $\mathrm{X}$ & $\mathrm{X}$ & $\mathrm{X}$ & $\mathrm{X}$ \\
\hline 80.0 & $\mathrm{X}$ & $\mathrm{X}$ & $\mathrm{X}$ & $\mathrm{X}$ & \\
\hline 100.0 & $\mathrm{X}$ & $\mathrm{X}$ & $\mathrm{X}$ & $\mathrm{X}$ & $\mathrm{X}$ \\
\hline
\end{tabular}

\subsection{PIV Procedures}

PIV is a technique for making instantaneous measurement of the velocity of particles within a flow field. It is an optical, essentially non-intrusive technique, and in its simplest form measures velocity in two dimensions. The basic principle is the 
measurement of the distance traveled by a given particle between two instants in time. Practical application of this principle requires statistical cross-correlation of discrete windows, and so it is the displacement of these windows, rather than individual particles, that is being measured.

\subsubsection{PIV Calculation}

Before any calculations can be done, the images must be transformed to correct for distortion as well as to apply a physical scale. The transformation comes as a result of the calibration procedure performed in Section 3.3.3.

Sometimes images are pre-processed, to mask out solid objects, reduce noise or otherwise improve the image quality before PIV processing. No pre-processing was necessary, or performed on any of the images presented herein.

The PIV calculation itself then follows. First, both images are divided into interrogation windows (IWs), usually squares. To take advantage of the Fast Fourier Transform (FFT) operation, IWs with side lengths equal to powers of two are chosen. In this case, multiple IW sizes were used iteratively. The smallest IW size, used for all data presented in this research, was $64 \times 64$ pixels. The selection of the smallest IW size is related to the selection of interframe time, as discussed in Section 3.3.1. Though cross-correlation works by comparing abstract patterns, it is simpler to consider that the particles themselves are being tracked. In this mindset, some particles will always move out of a given IW between the first and second laser pulses, i.e. between the first and second images. A judicious choice of the interframe time will restrict particle movement to about $1 / 4$ of the IW size. 
To ensure that the fewest number of particles are lost "out of the window," the entire cross-correlation process is first performed with a larger IW size, (in this case $128 \times 128$ pixels) with the cross-correlation calculation including one half IW-width in all directions around the IW under consideration. This is shown in Figure 3.17a). To improve the signal-to-noise ratio and thus the accuracy of the results, a second pass with the larger IW size of $128 \times 128$ pixels is performed. For this pass the second image is first deformed according to the velocity gradients measured during the first pass and the pixel intensities are recalculated using a bi-linear interpolation. This pixel-wise deformation is performed over the entire image, and then applied to each IW as necessary. This usually results in a stronger, more symmetric correlation peak in flows with high shear. The standard crosscorrelation is then performed one half IW-width in all directions, this time centred on the resultant vector provided by the first pass, as shown in Figure 3.17b). 


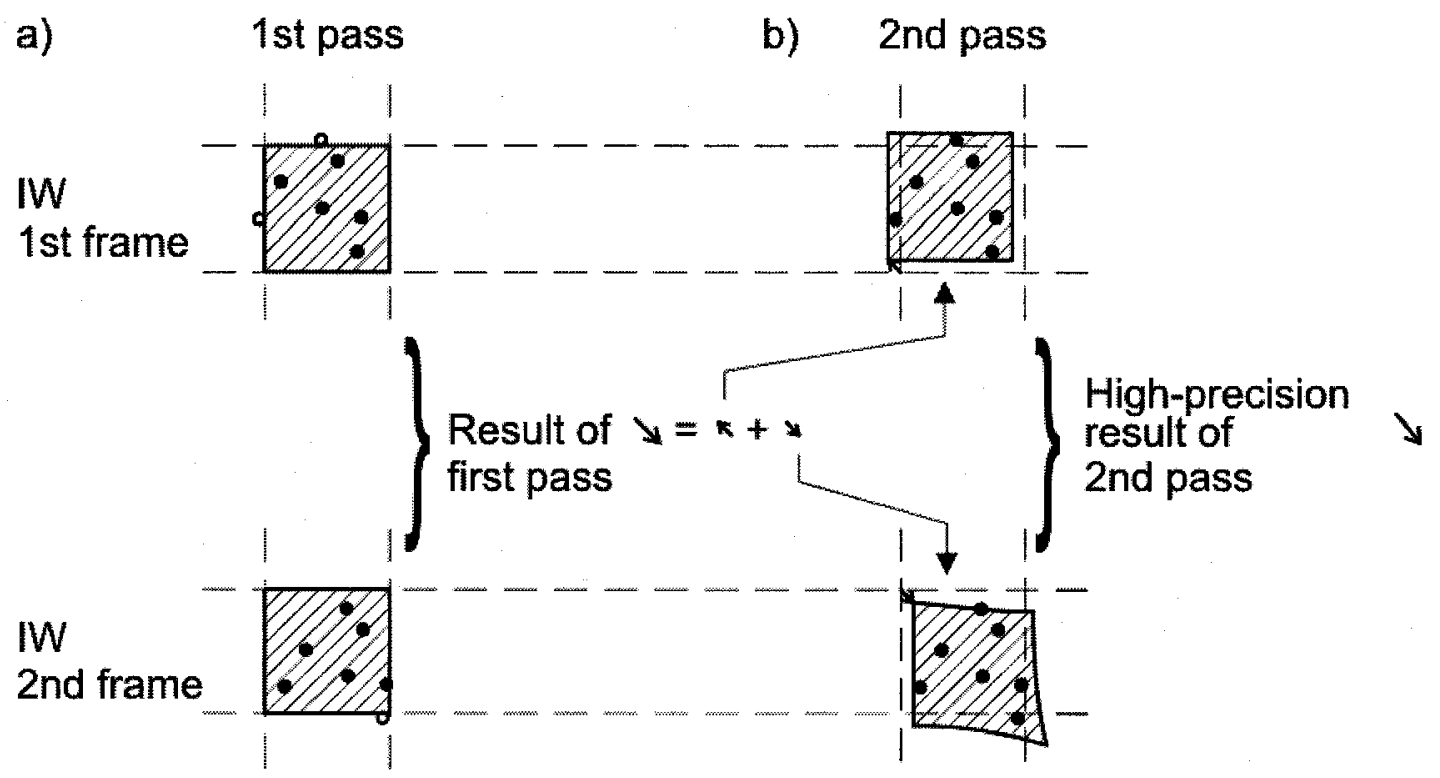

Figure 3.17-a) The initial pass searches $1 / 2$ the IW size in all directions and returns an approximate velocity vector. b) The result of this cross-correlation calculation, as well as those in surrounding IWs, is used as a reference vector field for the next pass, improving the signal to noise ratio.

The Standard $I 1 * I 2$ cross-correlation function provided by the FlowMaster software was used. The software uses an unspecified algorithm that is "similar but not exactly the same as the mathematical true correlation" (LaVision, 2006b) represented by:

$$
C(d x, d y)=\sum_{x=0, y=0}^{x<n, y<n} I_{1}(x, y) I_{2}(x+d x, y+d y),-\frac{n}{2}<d x, d y<\frac{n}{2}
$$

where $n$ is the side length of the IW and $I_{1}$ and $I_{2}$ are the image intensities at a given location. $C$ represents the strength of the correlation for a given shift, $d x, d y$. As specified earlier, the correlation function was restricted to displacements equal to half of the IW side length, or $n / 2$. In actuality, the correlation is calculated as if the IWs were repeated in space. From this the complex 2D FFT is calculated of each window. These are then multiplied, the complex conjugate is calculated, and after applying an inverse 
FFT this yields what is called the cyclic correlation function. In this way, a correlation map of all shifts up to $n / 2$ pixels is created up to 50 times faster than if the above equation were computed directly (LaVision, 2006b).

Unless otherwise specified, the shift with the greatest value of $C$, called the correlation peak, is assumed to represent the displacement of the IW under consideration. To better locate the centre of the correlation peak, a Gaussian function is fit to the correlation map in both the $x$ and $y$ direction in the immediate vicinity of the peak. A simplified correlation map is shown in Figure 3.18. The location of the maximum of this function is taken to be the true displacement of the IW. The resultant displacement vector multiplied by the magnification and divided by the interframe time represents the velocity of flow at the associated IW:

$$
\vec{V}=\frac{M \overrightarrow{\Delta X}}{\Delta t}
$$




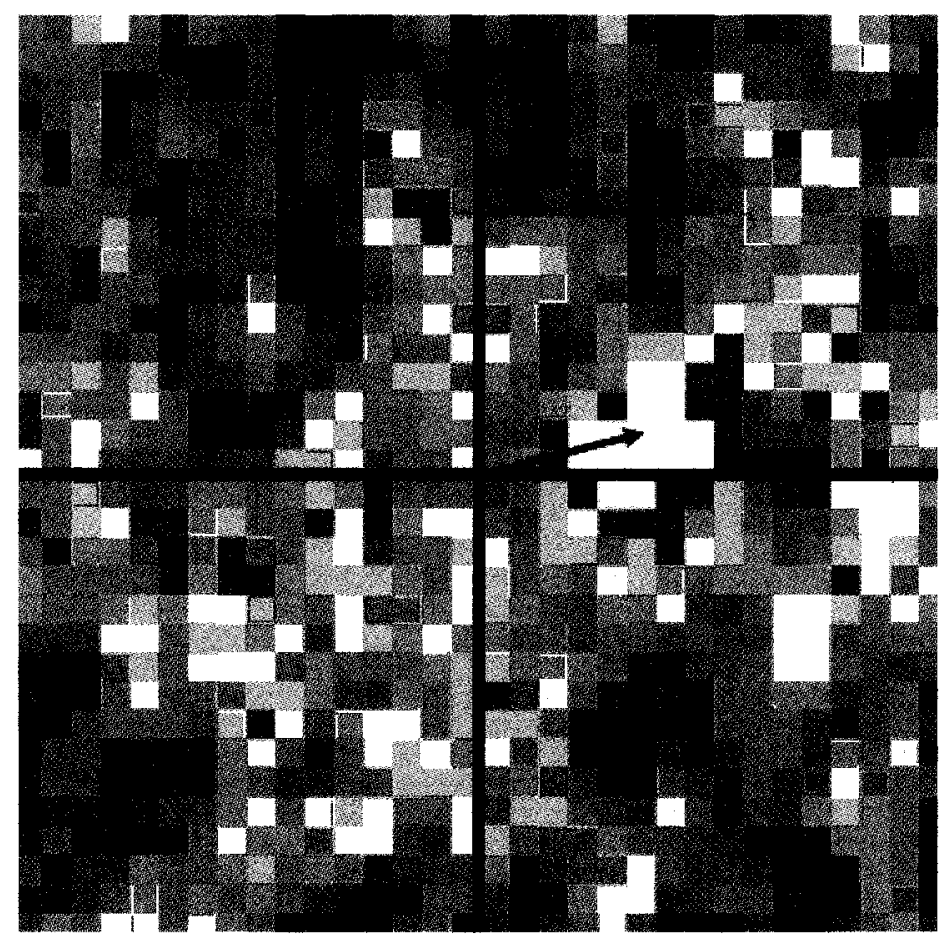

Figure 3.18 - An example correlation map. Fitting the correlation map to a Gaussian function in the vicinity of the peak allows the peak location (indicated by the arrow) to be determined with sub-pixel resolution.

This cross-correlation process is then repeated for the next IW, proceeding by rows and columns with shifts of $n / 2$ pixels between IWs. This represents an overlap of 50 percent in both the positive and negative $x$ and $y$ directions, producing an effective overlap of 100 percent and improving the spatial resolution by a factor of two. This is demonstrated in Figure 3.19, where the solid lines represent the initial grid of IWs and the dotted lines show the overlapping IWs. All of these steps are finally repeated with the final IW size of $64 \times 64$ pixels. With overlap, the final spatial resolution is 32 pixels.

As part of the multi-pass calculation process, the FlowMaster software automatically applies a median filter to each set of results before continuing on to the next pass. The 
use of median filters to improve PIV calculations and reduce the appearance of spurious vectors is described in depth by Westerweel (1994) and Nogueira et al. (1997).
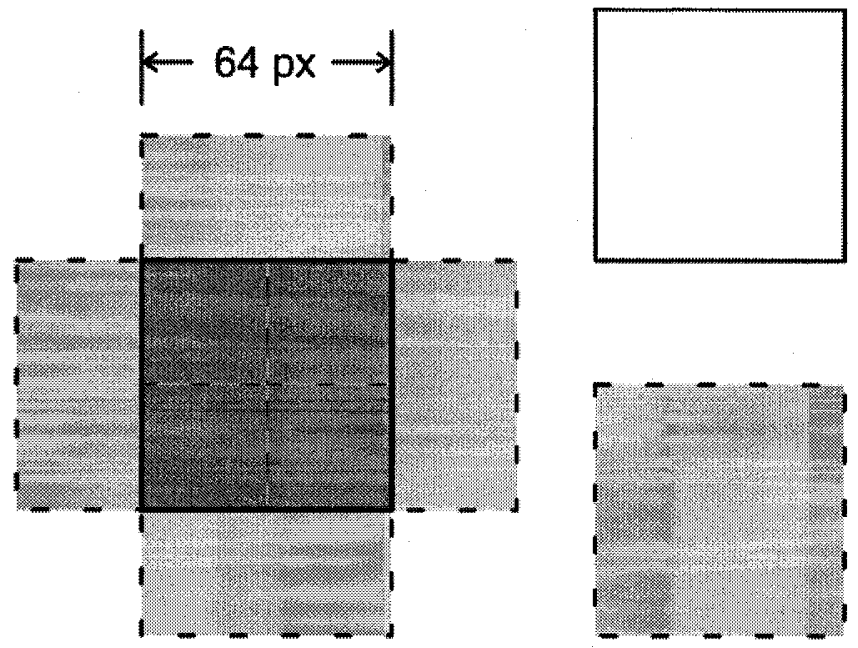

Interrogation window

Neighbour

(with $50 \%$ overlap)

Figure 3.19 - IWs are overlapped to increase the resolution of the resulting vector flow field.

Thus the PIV cross-correlation is applied a total of four times throughout the measurement domain. Twice with $128 \times 128$ pixel IWs spaced 64 pixels apart, and twice with $64 \times 64$ pixel IWs spaced 32 pixels apart.

\subsubsection{Post-processing}

Once the vector velocity flow field has been calculated for all IWs in a given image pair, post-processing was required to eliminate spurious vectors. The general definition of a spurious vector is any vector that does not represent the true velocity at its location. In the current work, spurious vectors were most often present: i) at the edges of the field of view, where no particles were present, and ii) close to the mouthpiece, where the optical density of the spray was quite high thus preventing the correlation function from distinguishing one IW from the next creating a correlation ridge rather than a single peak. 
Care was taken to minimize the use of post-processing, both in the number of operations used and the parameters specified for each, so as to not eliminate non-spurious vectors and bias otherwise good data.

The first post-processing step taken was to eliminate any vectors having a peak ratio of less than 1.2. The peak ratio as defined by the software manual (LaVision 2006b) is as follows:

$$
Q=\frac{P_{1}-m i n}{P_{2}-\min }
$$

The correlation values of the two highest peaks are represented by $P_{1}$ and $P_{2}$. The minimum value in the correlation map is used as min. Assuming that the strongest peak represents the true displacement, the peak ratio is similar to a signal-to-noise ratio. A peak ratio of 1 would thus represent two peaks of equal strength, making the choice of a displacement vector completely ambiguous. However, as explained in the software manual (LaVision 2006b), peak ratio rejection is a very unspecific post-processing method, often removing good vectors as well as bad. A peak ratio threshold of 1.2 was chosen, rejecting all vectors with peak ratios less than 1.2. This eliminated some vectors within the spray near the mouthpiece, where the spray was too optically dense to produce a single, distinct peak, as well as many vectors outside of the spray, where the correlation was based solely on noise.

The second post-processing step was chosen to eliminate many of the remaining vectors outside of the spray as well as some vectors within the spray which were obviously not physical (i.e. a vector with a magnitude of $150 \mathrm{~m} / \mathrm{s}$ directed into the 
mouthpiece). This step involved eliminating all vectors with streamwise velocities greater than $\pm 100 \mathrm{~m} / \mathrm{s}$ as well as all vectors with vertical velocities greater than $\pm 20 \mathrm{~m} / \mathrm{s}$. These thresholds were chosen after careful manual examination of a large sample of flow fields at all time delays.

In the final post-processing step, any groups of three or fewer vectors were eliminated. This operation was designed to eliminate any remaining vectors that were present outside of the spray. Figures 3.20 and 3.21 show a flow field before and after the peak-ratio rejection post-processing step. Many vectors are removed near the edges of the FOV, as well as one vector (indicated as (a) in the Figure 3.21) within the spray. The vector at (b) will be removed by the second, velocity-threshold rejection post-processing step. The single vector at (c) will be removed by the final post-processing step since it has no neighbouring vectors. The group of four vectors at (d) will not be removed by any post-processing step. 


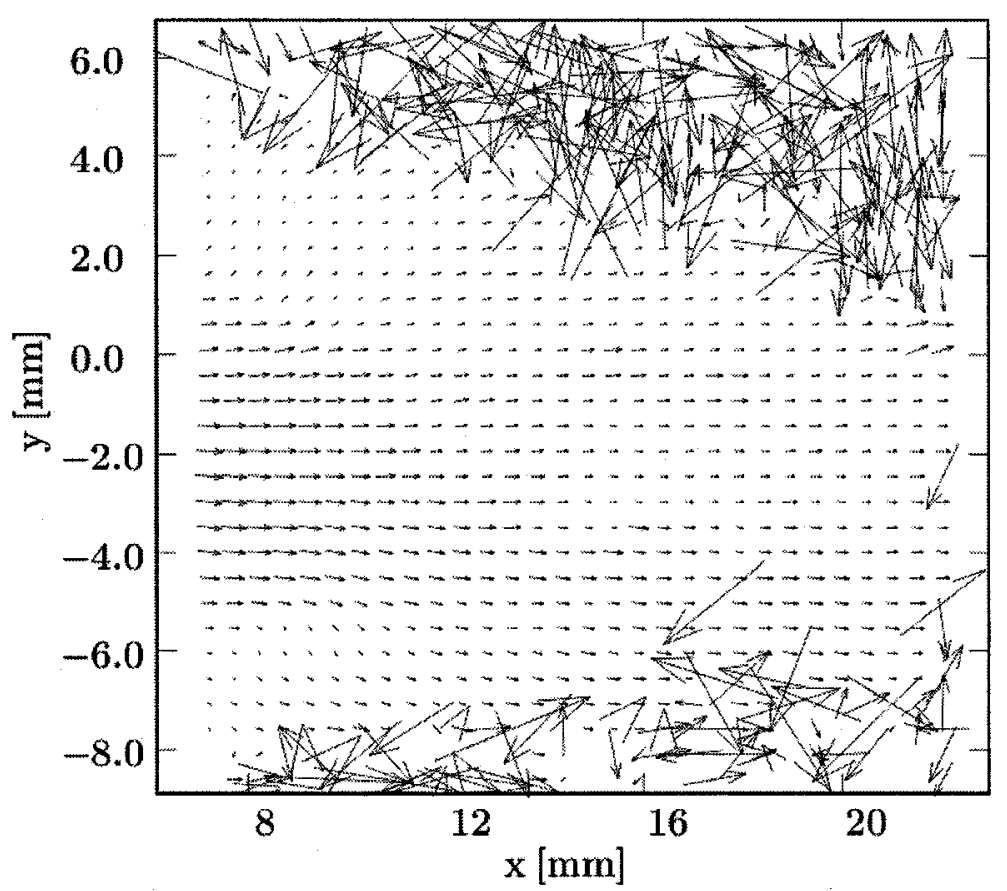

Figure 3.20 - A sample instantaneous vector velocity field before post-processing.

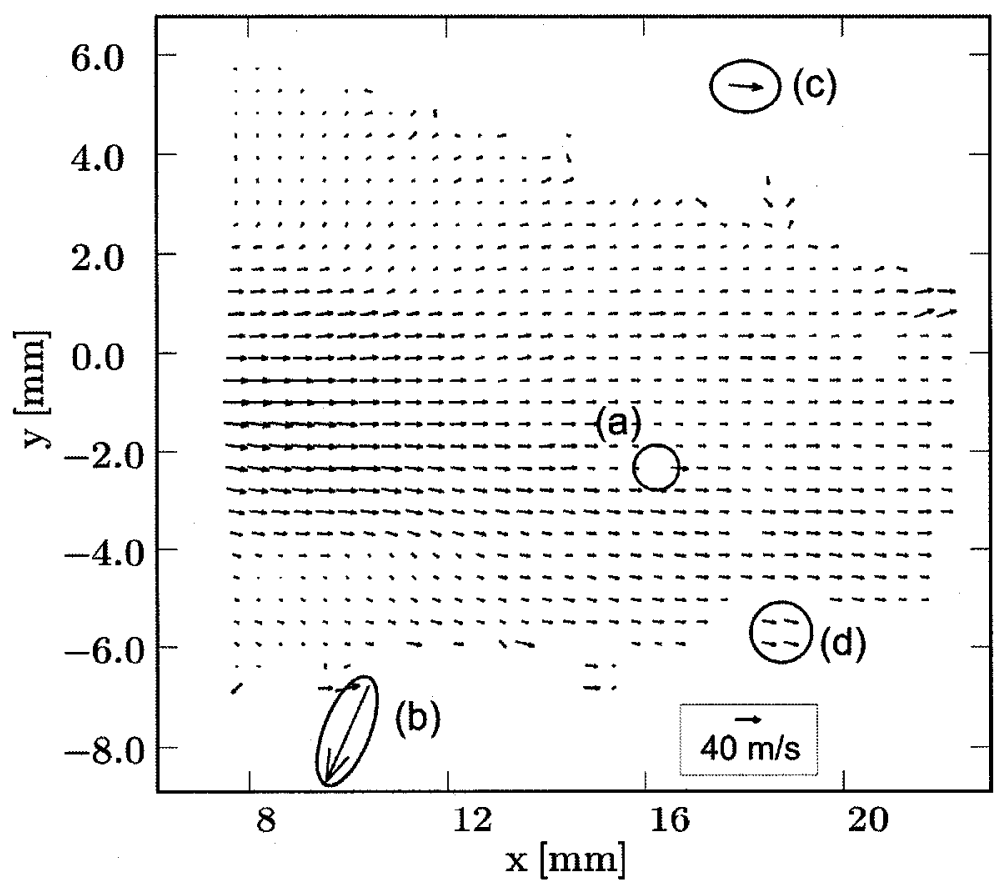

Figure 3.21 - Spurious vectors remaining after peak-ratio rejection. A vector was removed at (a). The vector at (b) will be removed by the velocity-rejection step. The vector at (c) will be removed by the small-groups step. The vectors at (d) will not be removed. 
Because a large number of grid points do not contain valid vectors after postprocessing, care must be taken during the averaging of vector fields to ensure every average vector is representative of the flow at a given point. This is controlled by specifying a minimum number of valid vectors that must be present at a given location before an average will be calculated at the location. This means that if few vector velocity flow fields contain valid vectors at the location marked (d) in Figure 3.21, no average will be presented at that point. It was decided that the vector velocity flow field from at least half of the actuations measured must contain a valid vector at a particular location before an average is calculated at that location. Figure 3.22 shows the number of vectors present at each location in the spray for a time delay of $1.3 \mathrm{~ms}$.

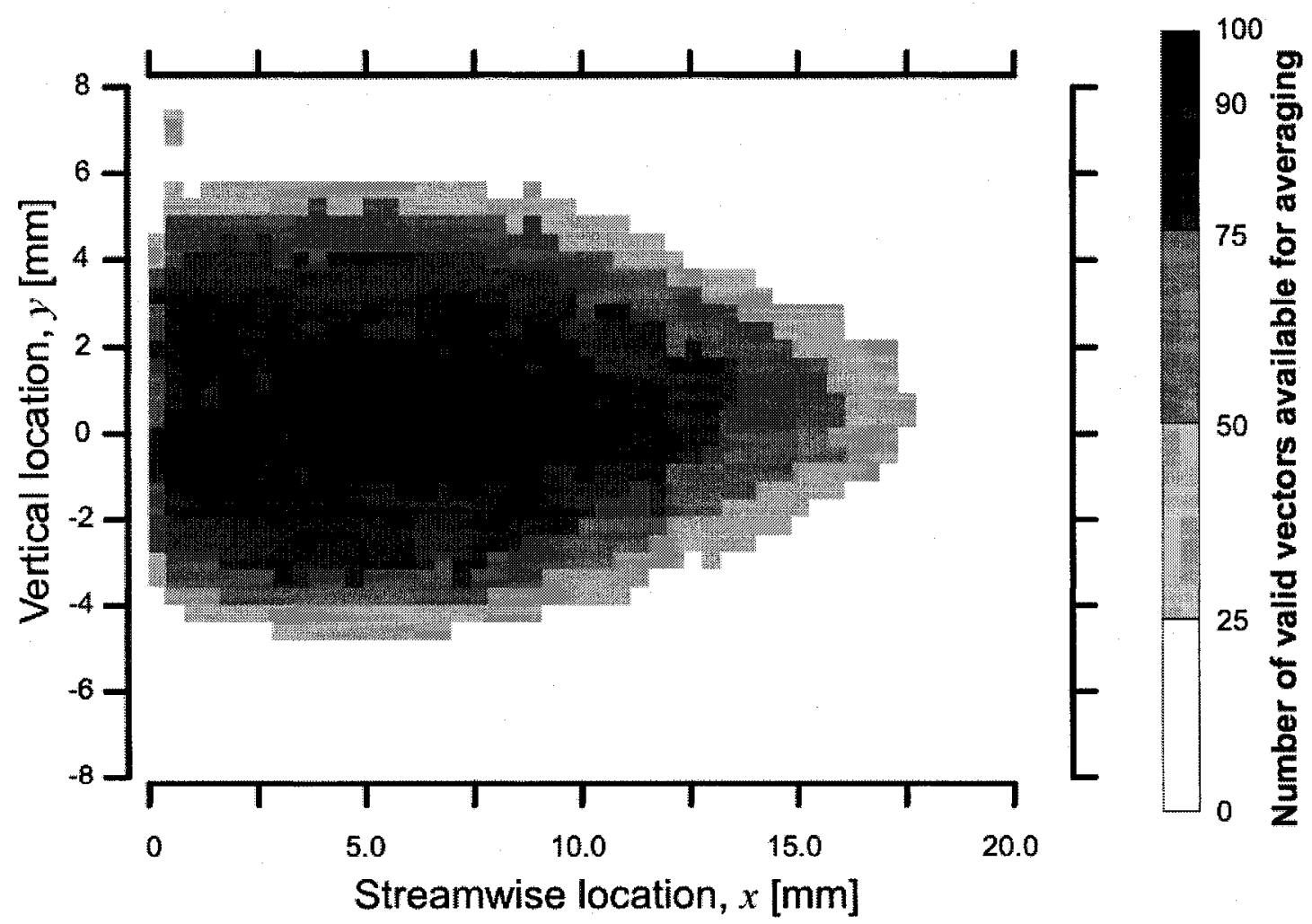

Figure 3.22 - The number of vectors remaining in the measurement region after postprocessing. Ventolin, open air, $1.3 \mathrm{~ms}$. 


\subsection{Spray Geometry Procedures}

The pMDI spray direction and cone angle were also calculated as part of the measurement procedure. These angles can be defined in two different ways. Both methods were used to compare and contrast the results they provided.

\subsubsection{Velocity method}

When velocity data is available, the location of the peak velocity can be used to determine the spray direction. The full-width, half-maximum (FWHM) criterion can then be used to determine the spray angle. On any given vertical profile of the spray, the spray edge is defined as the point where the velocity has decreased to half of the maximum velocity. In this way the spray edges at a number of streamwise locations can be plotted and curve-fitted. The angle between these edges is then defined as the spray angle. Similarly, the location of the peak velocity on a number of streamwise locations can be plotted, curve-fitted, and this curve defined as the spray direction.

A commercial software package (TableCurve2D, Systat Software Inc., Chicago, Illinois, USA) was used to fit the velocity profiles in order to determine the spray angles. After attempting to fit the data using a library of 3461 equations, the software determined that a $\chi^{2}$-distribution function provided the best fit.

A commercial mathematics software (Maple, Maplesoft, Waterloo, ON, Canada) was then employed to find the location of the peak as well as the two locations where the fitted equation had a value of half of the peak. These calculations were performed at four streamwise locations: $x=2.5 \mathrm{~mm}, x=7.5 \mathrm{~mm}, x=12.5 \mathrm{~mm}$, and $x=17.5 \mathrm{~mm}$. The 
locations of the spray peak and spray edge were then fitted linearly. The spray cone angle and spray direction were then measured using these lines, as shown in Figure 3.23.

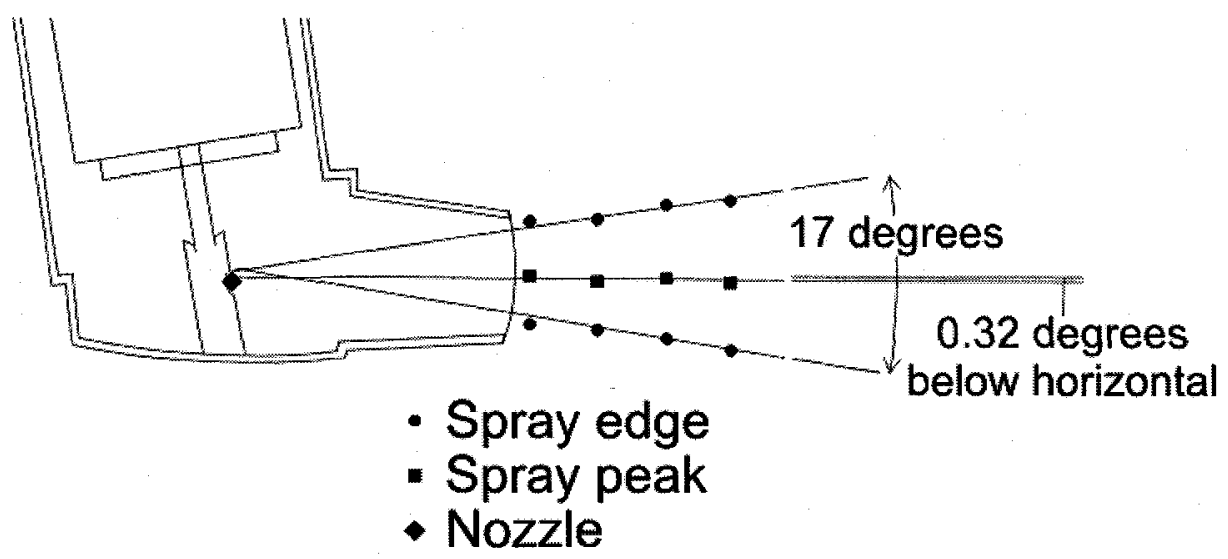

Figure 3.23 - The locations of the peak and half-peak velocities at four streamwise locations, as well as the location of the nozzle, are fitted linearly. The spray direction and angle are then calculated using the linear fits.

\subsubsection{Geometry method}

Spray direction and cone angle can also be defined based strictly on the images of the spray, as was calculated using an add-on software package to the PIV software called "Geometry" (LaVision GmbH, Gottingen, Germany). The user first selects an approximate spray direction and nozzle location. Also important is the selection of an intensity threshold. Pixels with intensities greater than the threshold are assumed to be showing spray particles; pixels with intensities less than the threshold are assumed to show only noise. As explained by the software manual (LaVision 2005) the algorithm then advances column-by-column in the streamwise direction. At each column it begins on the nozzle centreline and counts the number of pixels, $n_{P}$, containing spray particles. It then repeats the count, stopping once it has counted $m_{P}$ pixels containing spray 
particles. Rather than specifying $m_{P}$ directly, the user selects the fraction of particlecontaining pixels, $m_{P} / n_{P}$, desired to lie inside the spray edge. If the velocity varied linearly and the spray density was constant, selecting $m_{P} / n_{P}=0.5$ should give results identical to the FWHM criterion used for the velocity-based calculations. Though the velocity does not vary linearly, $m_{P} / n_{P}$ was still selected to be 0.5 . The algorithm then repeats this process on the same column, on the opposite side of the nozzle centreline before advancing to the next column. Once the spray edge locations are determined within a user-specified range of streamwise locations, they are linearly fitted. The range of streamwise locations to be measured is important only in cases where the spray does not occupy the entire field of view, as shown in Figure 3.24. This image was thus only analyzed from the left side of the screen to the black line in the figure. The ranges were selected so that the spray rarely ended before the cutoff point. The values selected are shown in Table 3.8. The angle between the two fitted spray edges is defined as the spray cone angle. The spray direction is then assumed to be centred between the two spray edges. An example, rotated $90^{\circ}$ clockwise (spray issuing from the top of the image), is shown in Figure 3.25. 


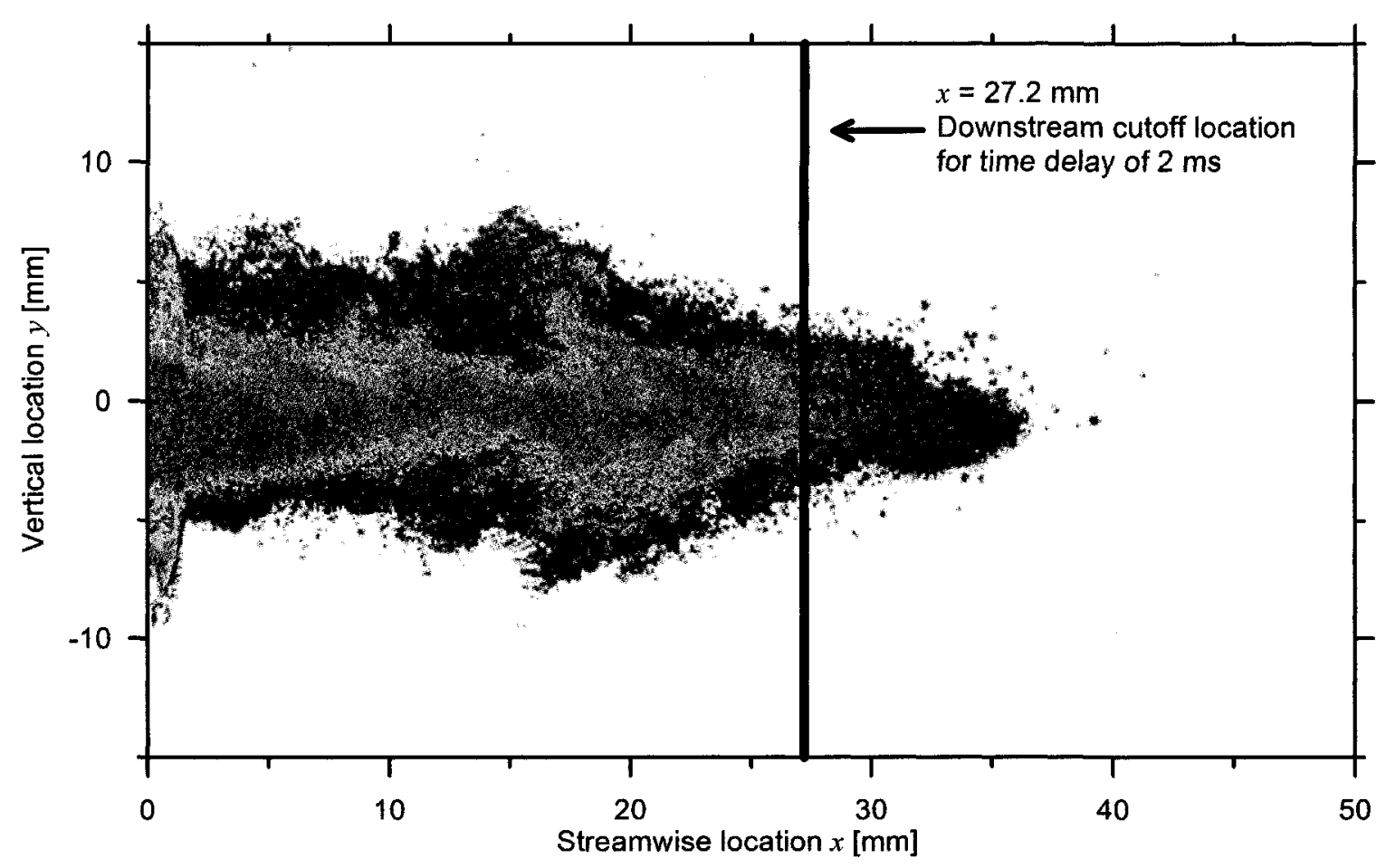

Figure 3.24 - A sample image showing the downstream cutoff location for spray at early time delays. ratio-Salbutamol HFA, 2 ms.

Table 3.8 - Downstream cutoff locations for geometry-based spray geometry calculation.

\begin{tabular}{cc}
\hline Time Delay [ms] & Cutoff Location [mm] \\
\hline 1.3 & 13.2 \\
2 & 27.2 \\
5 & 53.7 \\
$10-100$ & Entire field of view \\
\hline
\end{tabular}




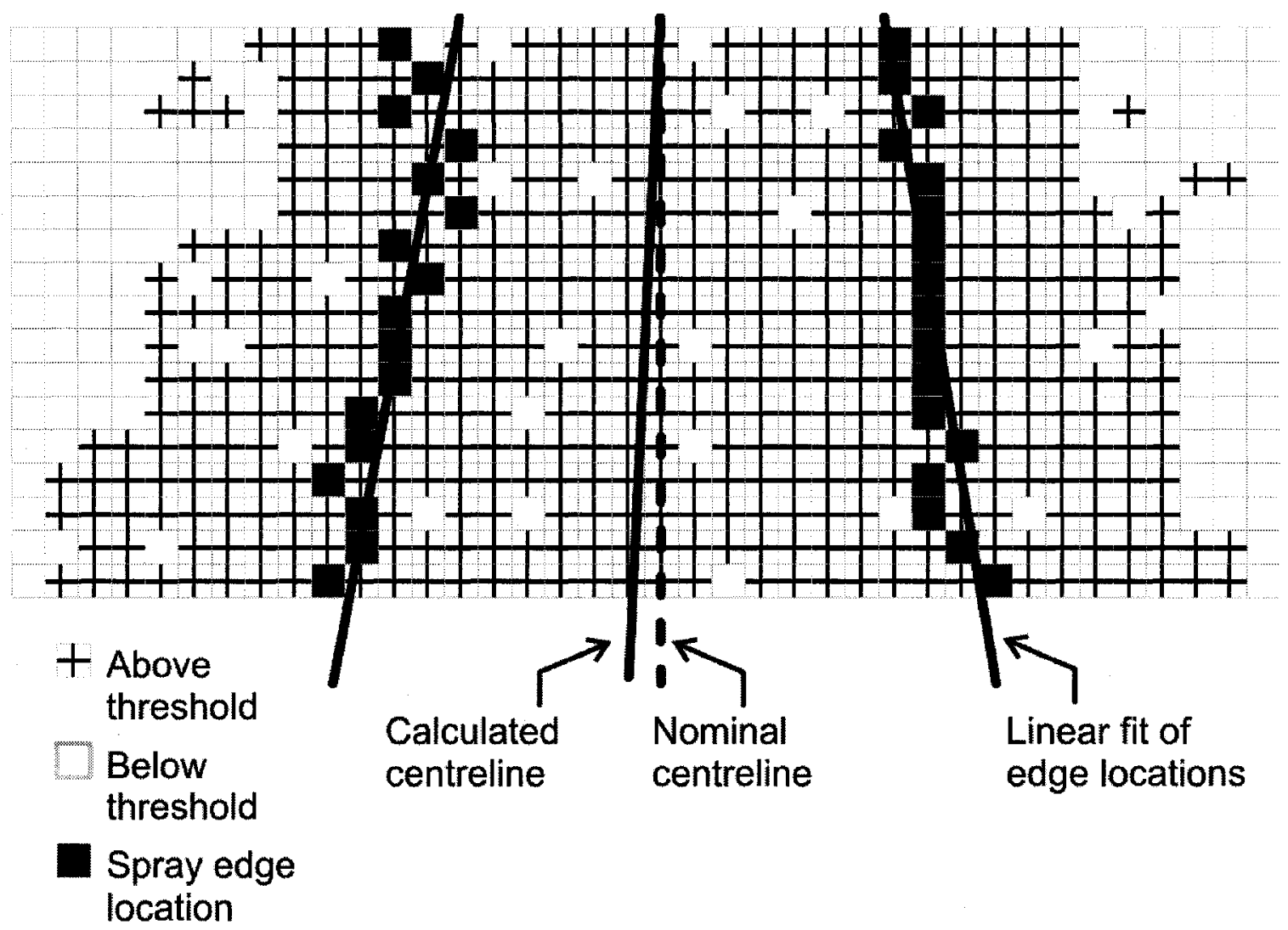

Figure 3.25 - The Geometry algorithm places the spray edge on a given row such that half of the pixels showing a spray are inside the spray and half are outside the spray. The centerline is calculated to be midway between the edge lines.

\subsection{Add-On Device Apparatus and Procedures}

Measurements were also made using a commercially available add-on spacer distributed under the name Aerochamber valved holding chamber with mouthpiece (Trudell Medical International, London, Ontario, Canada). To support the device during measurements, a steel bracket of adjustable height was manufactured and mounted to the optical table described in Section 3.2. A mould of the device mouthpiece was then made and glued to the bracket. This is shown in Figure 3.26. 
The calibration plate used for open air measurements was too large to be inserted inside the device, so a custom plate was designed. The custom plate consisted of a piece of paper containing a grid of circles $1.5 \mathrm{~mm}$ in diameter spaced $4 \mathrm{~mm}$ apart, printed on an inkjet printer at a resolution of 600 dots per inch. The paper target was glued to a piece of corrugated cardboard approximately $5 \mathrm{~mm}$ thick and cut to fit inside the spacer.

A first-surface mirror measuring $25 \mathrm{~mm}$ wide and $100 \mathrm{~mm}$ long was then mounted on a block of aluminum machined into a triangular-prism with angles of $45^{\circ}, 45^{\circ}$ and $90^{\circ}$ so as to reflect the laser sheet upward through the device. An alignment and calibration procedure similar to that described above in Section 3.3 was then followed.

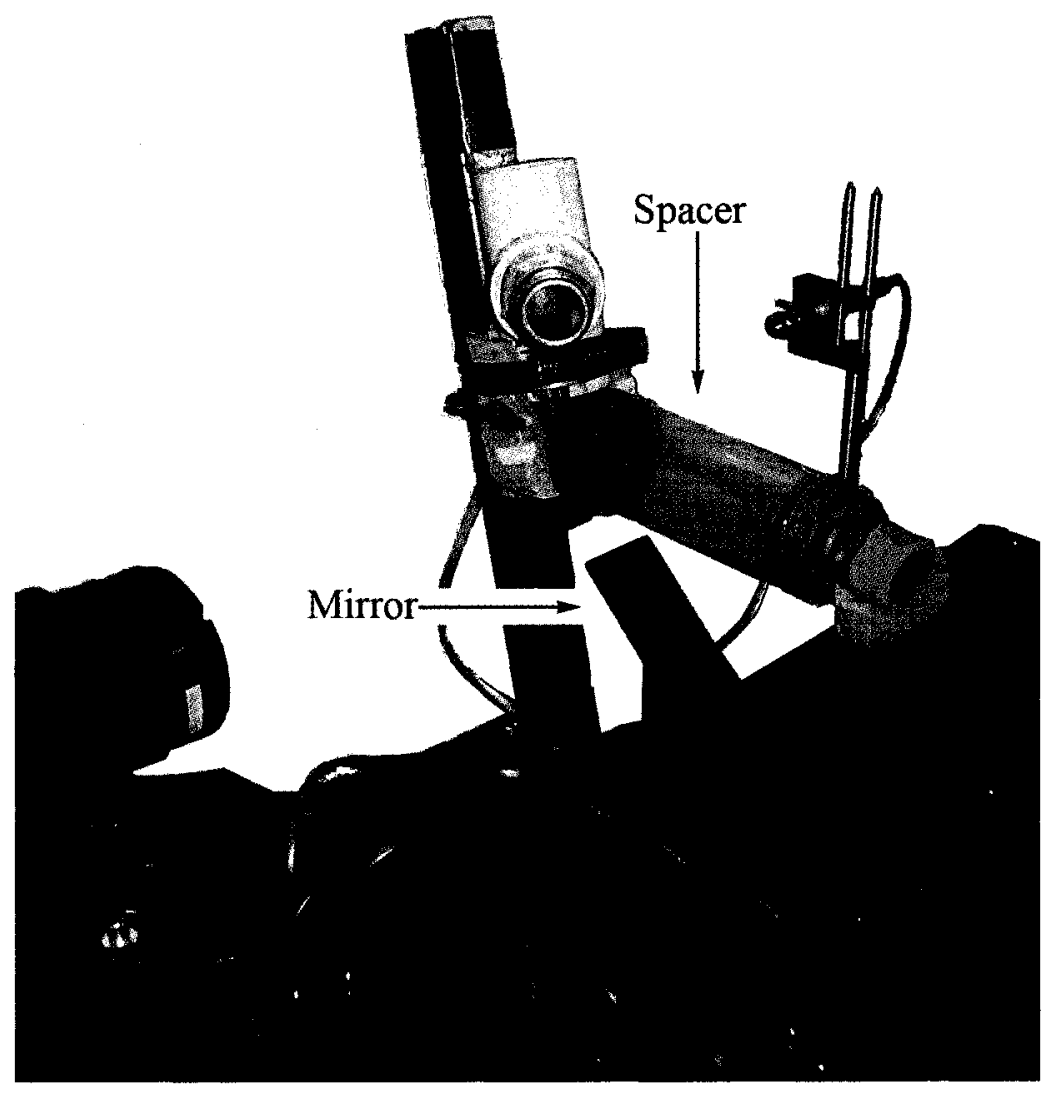

Figure 3.26 - The apparatus used to mount the add-on device. 


\subsection{Uncertainty Analysis}

There are always two and sometimes three measurements associated with every PIV image taken: the velocity, the time delay, and the flow rate of the co-flow. For each velocity field measurement there is uncertainty associated with the calculation of velocity, as well as an uncertainty in the amount of time elapsed since the pMDI was actuated. Only measurements taken with a co-flow of air have an uncertainty in the mass flow rate of air through the pMDI. Once these three measurements are made, there is also an uncertainty associated with the calculation of mean velocities.

\subsubsection{Uncertainty in Velocity Calculation}

PIV is a direct measurement technique, measuring displacement by simply taking a picture of the flow at two instants in time. Because of this, there are few elemental error sources, all having a direct effect on the basic calculation of velocity used in PIV:

$$
\vec{V}=\frac{\overrightarrow{\Delta X}}{M \Delta t}
$$

Where $\overrightarrow{\Delta X}$ represents the displacement in the image plane. All the identified elemental error sources are listed in Table 3.9, below. 
Table 3.9 - Listing of elemental errors associated with PIV measurements.

\begin{tabular}{lccc}
\hline & Error & Group & Type \\
\hline$e_{1}$ & Determination of magnification & Calibration & Precision \\
$e_{2}$ & Determination of image correction function & Calibration & Precision \\
$e_{3}$ & Non-zero laser pulse length & Data acquisition & Precision \\
$e_{4}$ & Laser timing & Data acquisition & Precision \\
$e_{5}$ & Noise & Data acquisition & Precision \\
$e_{6}$ & Loss of particles & Data reduction & Precision \\
$e_{7}$ & Pixel-locking & Data reduction & Bias \\
$e_{8}$ & Velocity gradient & Data reduction & Bias \\
$e_{9}$ & Traceability of seed particles & Data acquisition & Precision \\
\hline
\end{tabular}

The estimation of the contribution of each of these terms, as well as their contribution toward the total measurement uncertainty, is described in Appendix A and summarized in Table 3.10, below.

Table 3.10 - Estimation of elemental errors.

\begin{tabular}{ccc}
\hline Error & Variable & Value \\
\hline$e_{1}$ & $\delta \mathrm{M}$ & 0.0132 \\
$\mathrm{e}_{2}$ & $\delta(\Delta \mathrm{X})$ & $1.57 \mu \mathrm{m}$ \\
$\mathrm{e}_{3}$ & $\delta(\Delta \mathrm{X})$ & $0.50 \mu \mathrm{m}$ \\
$\mathrm{e}_{4}$ & $\delta(\Delta \mathrm{t})$ & $50 \mathrm{~ns}$ \\
$\mathrm{e}_{5}$ & $\delta(\Delta \mathrm{X})$ & $2.48 \mu \mathrm{m}$ \\
$\mathrm{e}_{6}$ & & \\
$\mathrm{e}_{7}$ & $\delta(\Delta \mathrm{X})$ & -- \\
$\mathrm{e}_{8}$ & $\delta(\Delta \mathrm{X})$ & -- \\
$\mathrm{e}_{9}$ & $\delta(\Delta \mathrm{X})$ & -- \\
\hline
\end{tabular}

Among the last four errors, $\mathrm{e}_{6}$ is is commonly lumped (Boillot and Prasad, 1996 and Prasad et al., 1992) with with $e_{5}$ while $e_{7}, e_{8}$ and $e_{9}$ can be considered negligible, leaving only precision errors to be propagated. The total uncertainty can thus be described by: 


$$
\delta v=\sqrt{\left(\frac{\partial v}{\partial M}\right)^{2}\left(\mathrm{e}_{1}\right)^{2}+\left(\frac{\partial v}{\partial(\Delta X)}\right)^{2}\left(\mathrm{e}_{2}^{2}+\mathrm{e}_{3}^{2}+\mathrm{e}_{5 / 6}^{2}\right)+\left(\frac{\partial v}{\partial(\Delta t)}\right)^{2}\left(\mathrm{e}_{4}\right)^{2}}
$$

After calculating the partial derivatives from equation 3.5 and using the values of the error terms calculated in Appendix A and shown in Table 3.10, this simplifies to:

$$
\delta V=\sqrt{\left(-\frac{\Delta X}{M^{2} \Delta t}\right)^{2}\left(1.74 \cdot 10^{-4}\right)+\left(\frac{1}{M \Delta t}\right)^{2}\left(8.63 \cdot 10^{-12} \mathrm{~m}^{2}\right)+\left(-\frac{\Delta X}{M \Delta t^{2}}\right)^{2}\left(6.2 \cdot 10^{-16} \mathrm{~s}^{2}\right)}
$$

The same interframe time of $1.2 \mu \mathrm{s}$ was used for all measurement cases, allowing displacement to be expressed in terms of velocity alone. Using the magnification that was calculated during the open-air trials $(M=0.500)$ as an example, the velocity uncertainty simplifies to a function of the velocity alone:

$$
\delta V=\sqrt{24.6\left(\frac{m}{s}\right)^{2}+0.00244 V^{2}}
$$

The absolute and percentage velocity uncertainties are shown in Figure 3.27 as functions of both velocity and pixel-displacement in the image plane. 


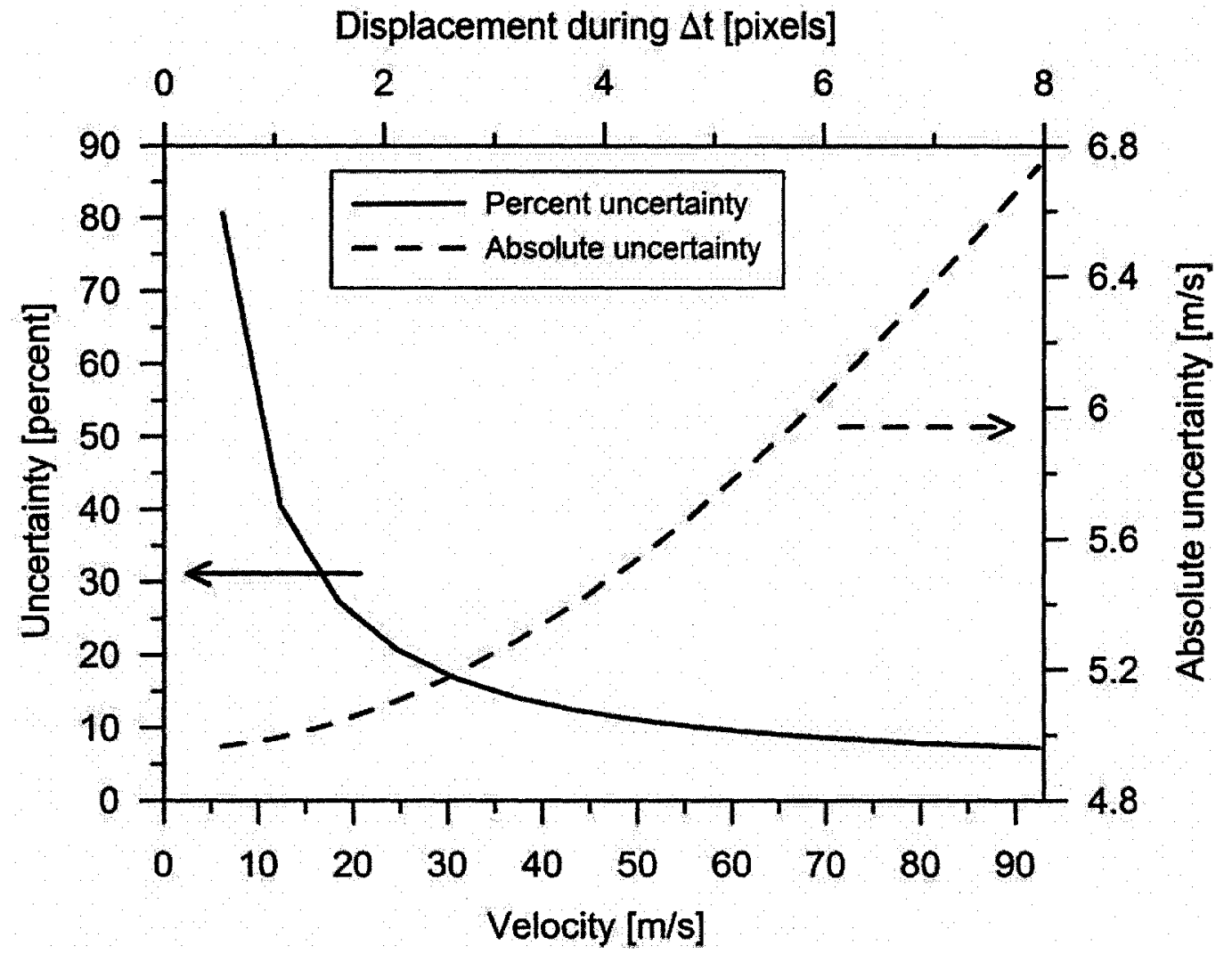

Figure 3.27 - The uncertainty in velocity expressed as a function of velocity.

\subsubsection{Uncertainty in Time Delay}

Every time the pMDI is actuated, a signal is sent to the PIV computer which then waits a specified amount of time before triggering the laser and cameras. The uncertainty involved in this process was assumed to be negligible compared to the specified time delays, so the entire length of this process can be considered bias error. The elemental errors in the time delaying process can be broken down as outlined in Table 3.11: 
Table 3.11 - Elemental errors associated with data acquisition triggering.

\begin{tabular}{cccc}
\hline & Error & Group & Type \\
\hline$e_{1}$ & He-Ne laser beam travels from canister to photodiode & Data acquisition & Bias \\
$e_{2}$ & Maximum possible photodiode response time & Data acquisition & Bias \\
$e_{3}$ & Voltage inverter response time & Data acquisition & Bias \\
$e_{4}$ & Time for signal to travel to PIV computer & Data acquisition & Bias \\
$e_{5}$ & Resolution of PIV timing card & Data acquisition & Precision \\
$e_{6}$ & Operation of metering valve & Data acquisition & Precision \\
\hline
\end{tabular}

The estimation of each of these elemental errors is described in Appendix B. Assuming the worst possible case for all of these values, and summing all of these errors directly, a total of $101 \mathrm{~ns}$ would be added onto the time delay. Since the shortest time delay selected is $1.3 \mathrm{~ms}$, these terms can be safely neglected.

\subsubsection{Uncertainty in Mass Flow Rate}

The mass flow controller used in the measurements with a co-flow has an accuracy of $\pm 1 \%$ of its full-scale, corresponding to \pm 1 SLPM. It also has a repeatability of $\pm 0.05 \%$ of its full-scale, corresponding to \pm 0.05 SLPM. These combine to yield a total uncertainty of $\mathrm{e}_{\mathrm{Q}}=1.0$ SLPM. Thus the mass flow rate used for all measurement cases incorporating a co-flow is $30 \pm 1$ SLPM.

\subsubsection{Uncertainty in Averaging the Velocity Fields}

To calculate the mean velocity field at a given time delay, the velocity from many instantaneous velocity fields was averaged. To determine the number of instantaneous measurements required, $N$ measurements are first taken and the mean streamwise velocity, $u_{\mathrm{N}}$, is calculated at a given point. The mean velocity at the same point is then 
calculated using $n$ measurements, $u_{\mathrm{n}}$, where $n<N$. A plot of $u_{\mathrm{n}} / u_{\mathrm{N}}$ versus $n$ can then be used to approximate the number of measurements required to sufficiently represent the true mean. This is shown in Figure 3.28 for two points in the measurement field at three time delays and $\mathrm{N} \approx 100$.

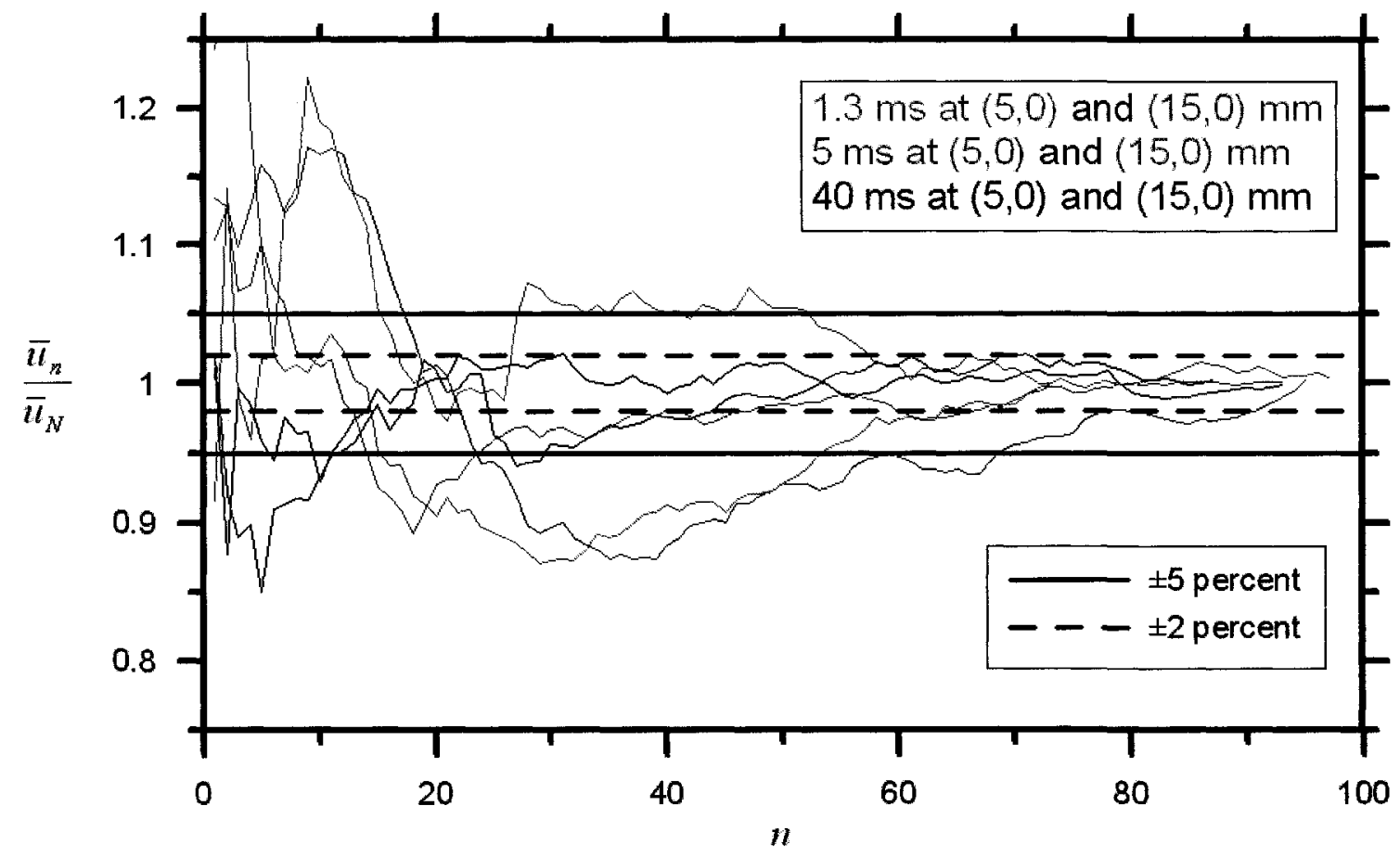

Figure 3.28 - Normalized running average for streamwise velocity, $u$.

It can be clearly seen that after taking 70 measurements, the velocities have all converged to within $\pm 5 \%$ of the final average, and that by 90 measurements this falls to $\pm 2 \%$. For this reason it was decided to take 100 measurements for each measurement condition at each time delay.

Once the mean velocity has been calculated, the spread of the measurements around this mean can be quantified. Figure 3.29 shows the distribution of velocity measurements at the same time delays and locations as Figure 3.28, above. Though the measurements 
appear to be normally distributed, the nature of the physical event indicates that the distribution is in fact bounded at 0 and at a maximum velocity. The spread in the measured data is assumed to be due not to measurement uncertainty, but to variation in the spray velocities from one actuation to another, possibly due to the metering valve operation as mentioned in Table 3.11 and discussed in Appendix B.6. For this reason the variance of the velocity measurements is reported independent of the uncertainty, in Appendix C. 
(a) Time Delay of $1.3 \mathrm{~ms}$

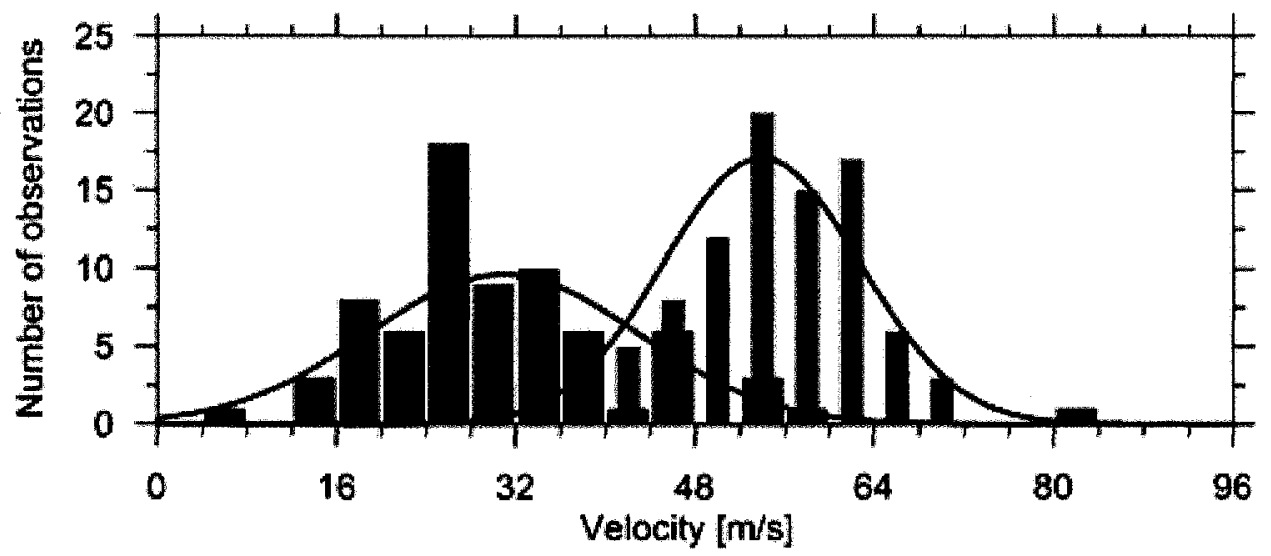

(b)

Time Delay of $5 \mathrm{~ms}$

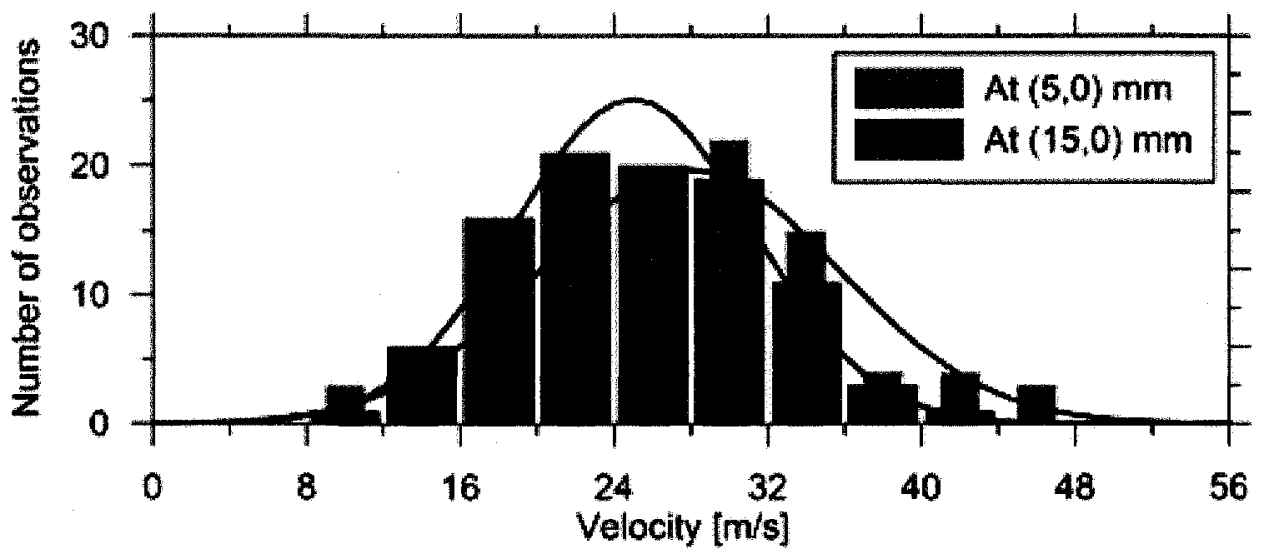

(c) Time Delay of $40 \mathrm{~ms}$

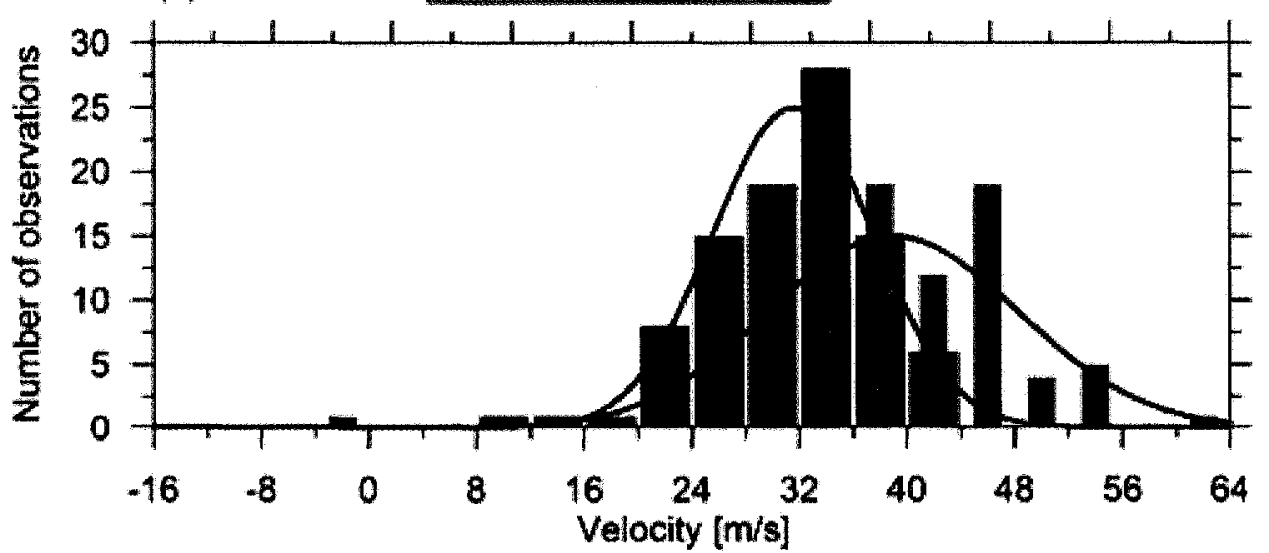

Figure 3.29 - Histograms of measurements of streamwise velocity, $u$, at two locations and three time delays. Overlaid with a corresponding normal distribution for comparison. 


\section{Chapter 4: Experimental Results}

\subsection{Introduction}

To the author's knowledge, the results presented here are the most detailed spatial measurements of the pMDI spray available in the open literature. The use of the PIV technique provided large-area instantaneous vector velocity measurements at a spatial resolution of $0.4 \mathrm{~mm}$. The PIV system has very poor temporal resolution, and is limited to measuring one instant in time per pMDI actuation. This forced the temporal resolution to be restricted to ten discrete instants in time relative to pMDI actuation.

Due to the many similarities observed under the different flow conditions studied, only the first, base-case condition of a pMDI actuated into open, quiescent air will be presented in depth. Subsequent sections will discuss only the similarities and differences between the condition being discussed and the base-case.

\subsection{Spray Issued Into Open, Quiescent Air}

The spray issued from a pMDI into open, quiescent air enters the measurement area approximately $0.8-1.0 \mathrm{~ms}$ after the data acquisition system is triggered. The leading edge of the spray leaves the measurement area approximately $1.0 \mathrm{~ms}$ later. The variation in the spray pattern among different actuations is significant, as is evident in Figure 4.1, which shows a comparison of two spray events, both imaged at a time delay of $1.7 \mathrm{~ms}$ 
after actuation. There is also noticeable variation in the tip penetration distance in cases where the leading edge of the spray is visible within the FOV. This is likely due to slight variations in the flow through the expansion chamber and nozzle from one actuation to the next. Figure 4.2 shows measured tip penetration of 70 measurements taken at a time delay of $1.7 \mathrm{~ms}$. Variations of $\pm 30 \%$ relative to a mean of $11.5 \mathrm{~mm}$ are possible from maximum to minimum, although the results appear normally distributed.

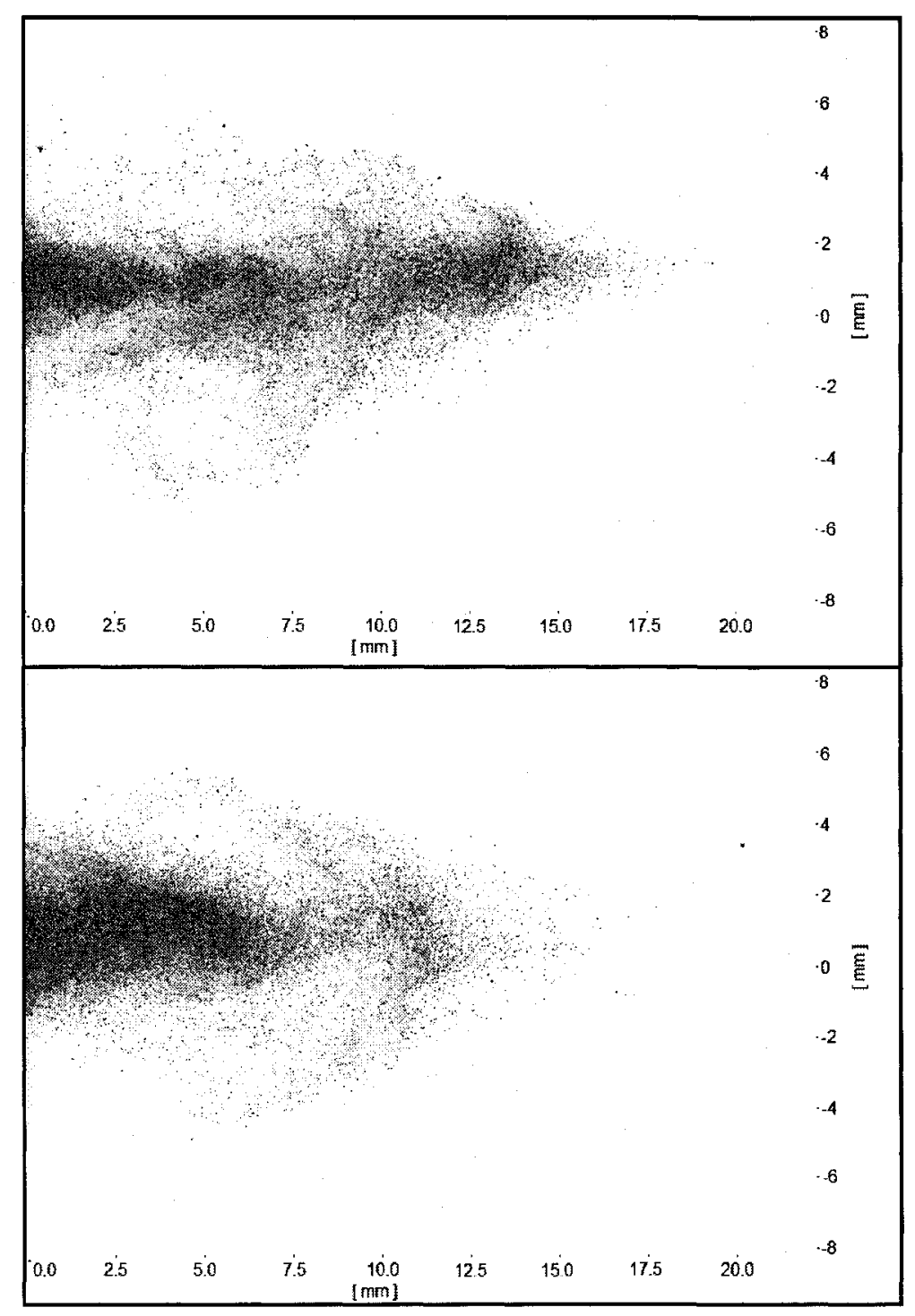

Figure 4.1 - Two images of the spray at a time delay of $1.7 \mathrm{~ms}$. 


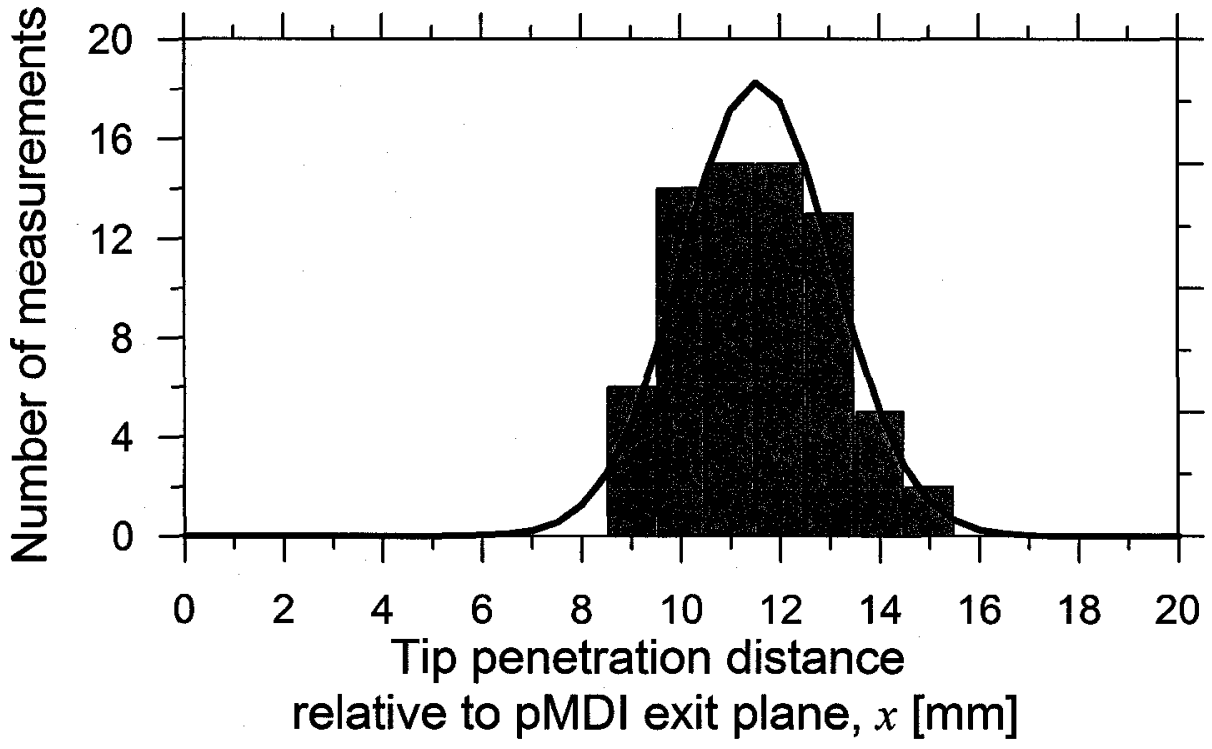

Figure 4.2 - Histogram of tip penetration taken at a time delay of $1.7 \mathrm{~ms}$, overlaid with a normal distribution having the same mean and standard deviation.

Two samples of the calculated vector flow field of individual actuations for a time delay of $2 \mathrm{~ms}$ are presented in Figure 4.3, further demonstrating the differences in velocity from spray to spray.

Once the vector velocity flow field from 100 actuations has been averaged, however, a clearer picture of the flow emerges. To better visualize the velocities present in these flows, it is useful to overlay vertical profiles of streamwise velocity at a number of streamwise locations in the spray. Figures $4.4-4.8$ show vertical profiles of streamwise velocity at streamwise locations of $x=2.5,7.5,12.5$ and $17.5 \mathrm{~mm}$ superimposed over the corresponding vector velocity flow fields for all time delays considered. 


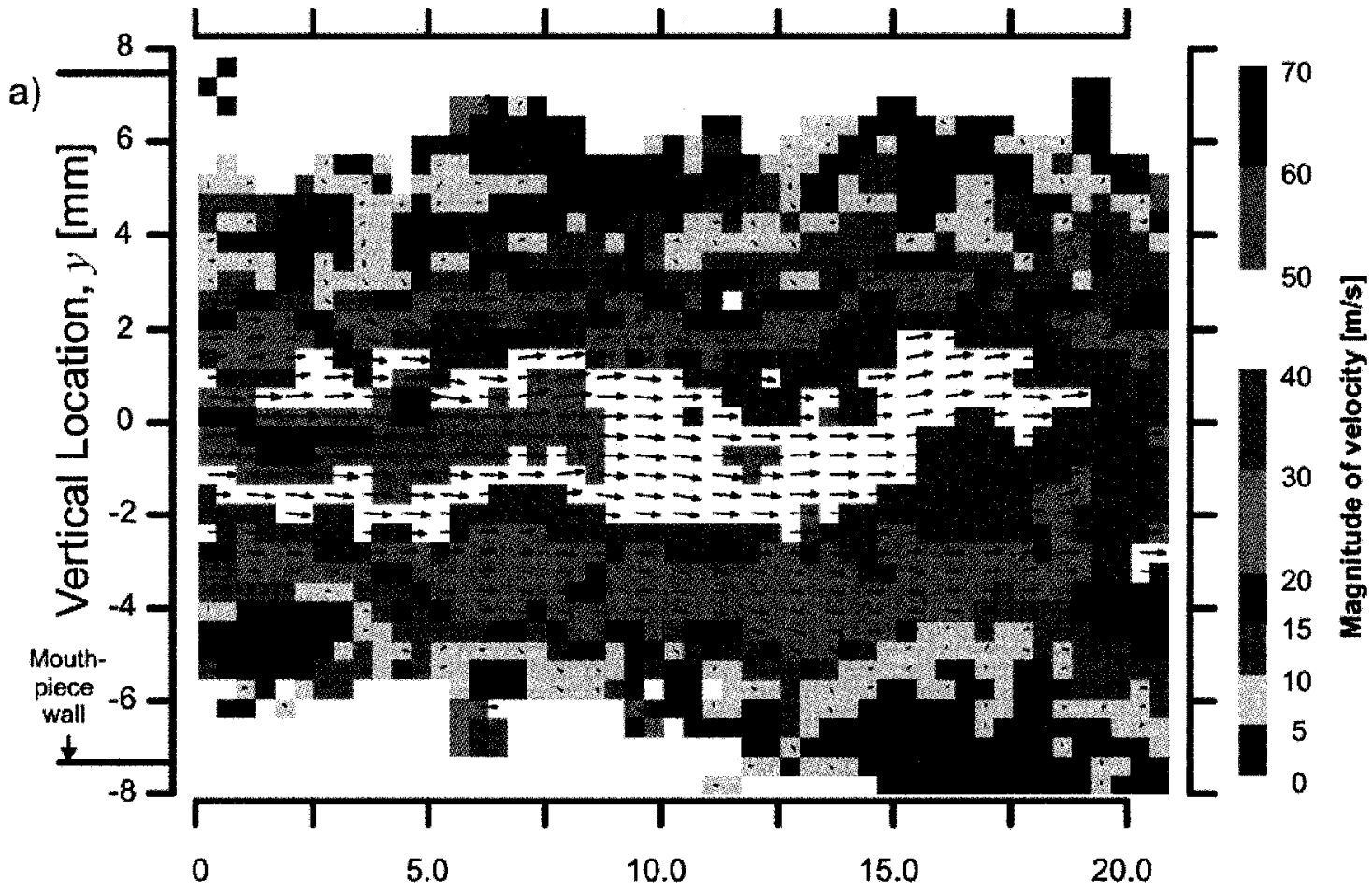

b)

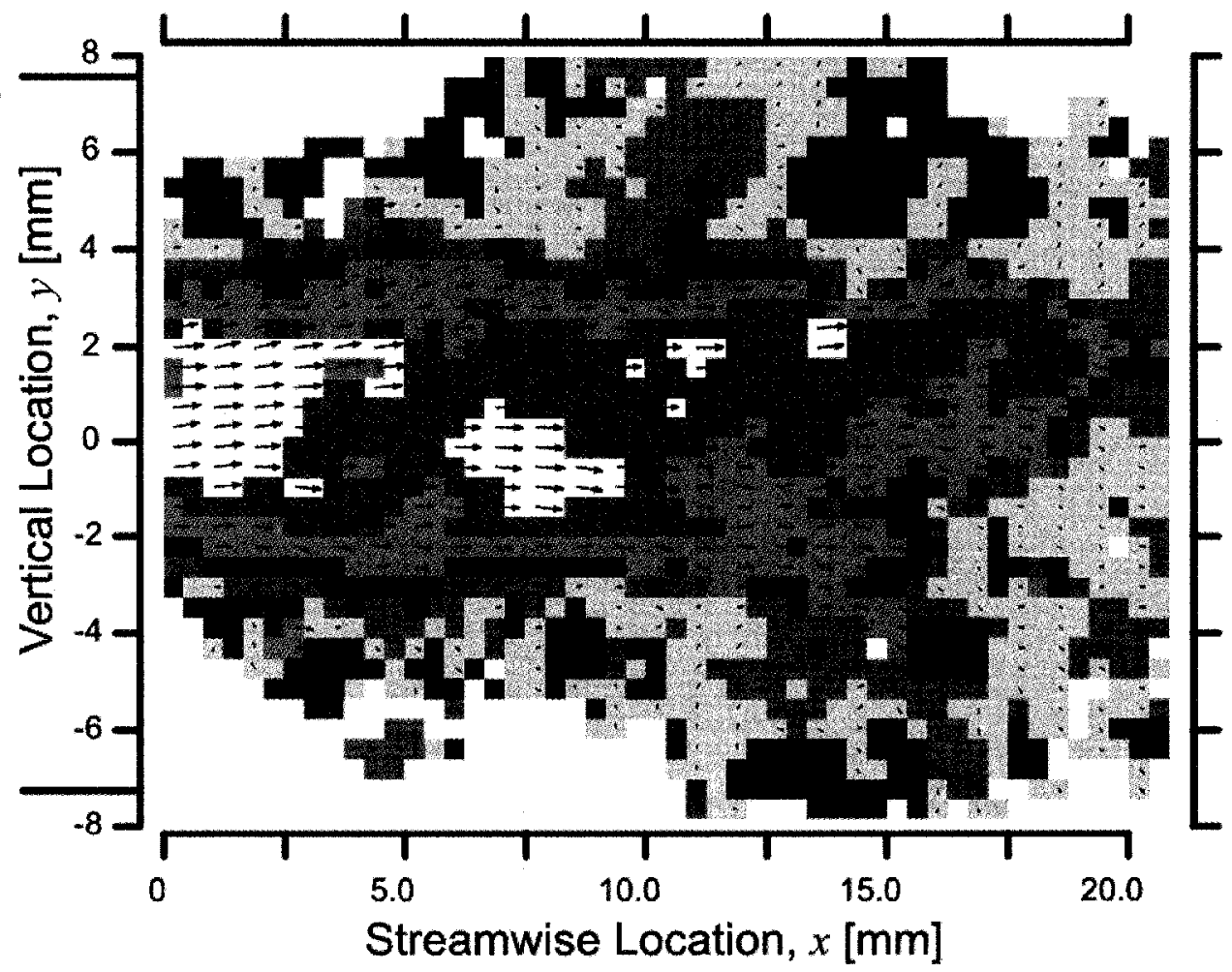

Figure 4.3 - Large differences in velocity are seen between two actuations, selected at random. Ventolin, open air, $2 \mathrm{~ms}$. 

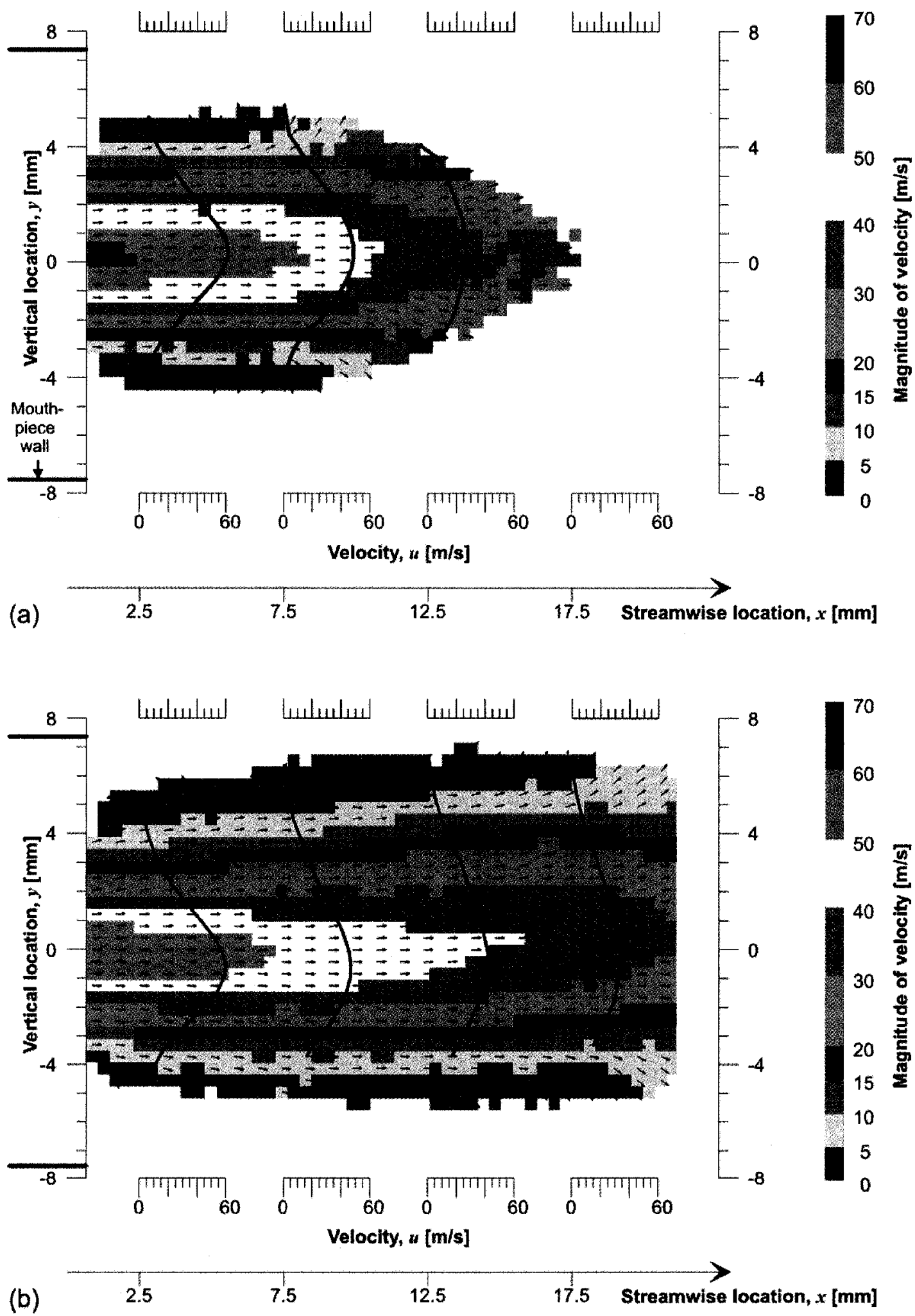

Figure 4.4 - Average vector velocity flow field. Ventolin, open air, (a) 1.3 and (b) 2 ms. 

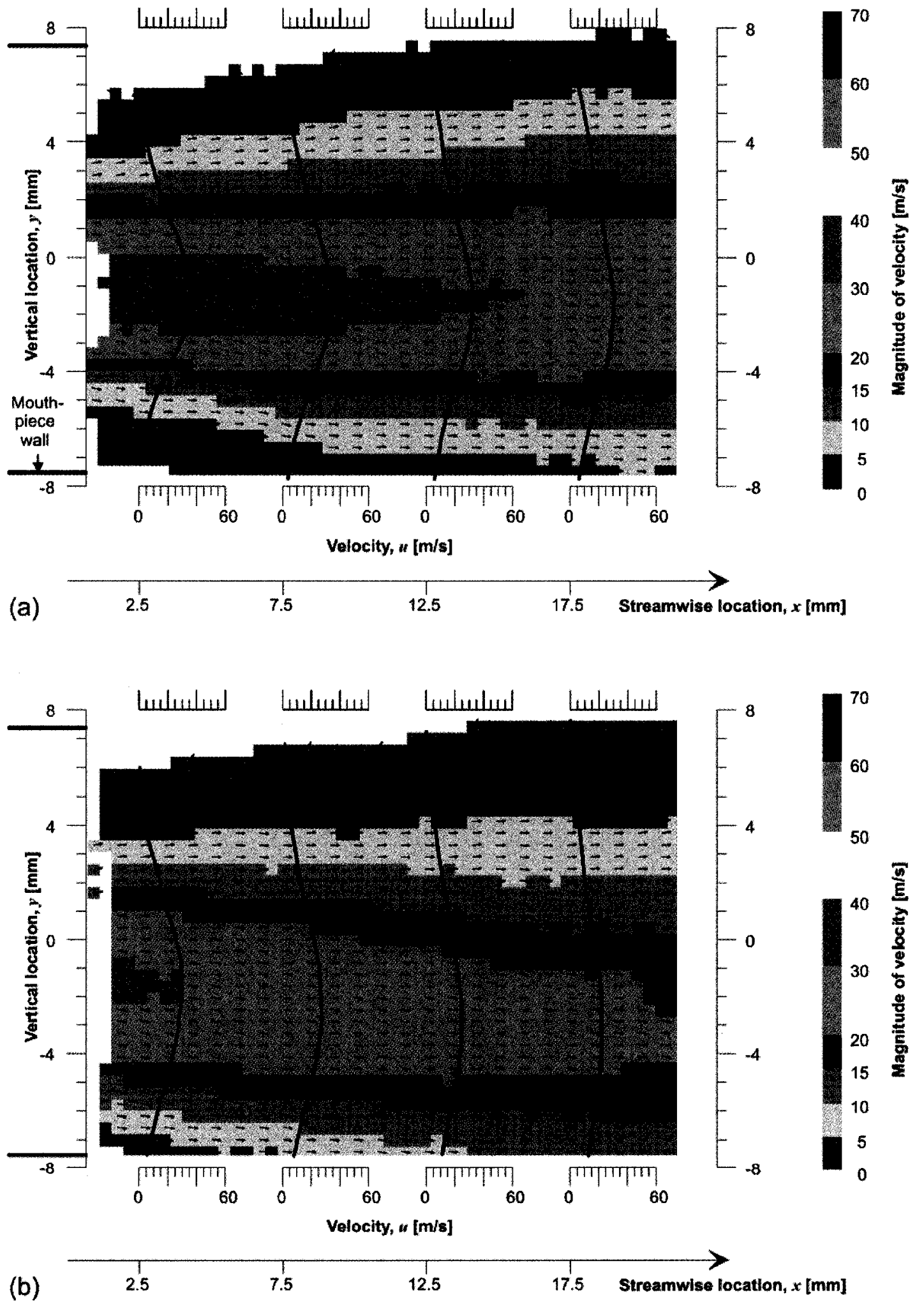

Figure 4.5 - Average vector velocity flow field. Ventolin, open air, (a) 5 and (b) $10 \mathrm{~ms}$. 

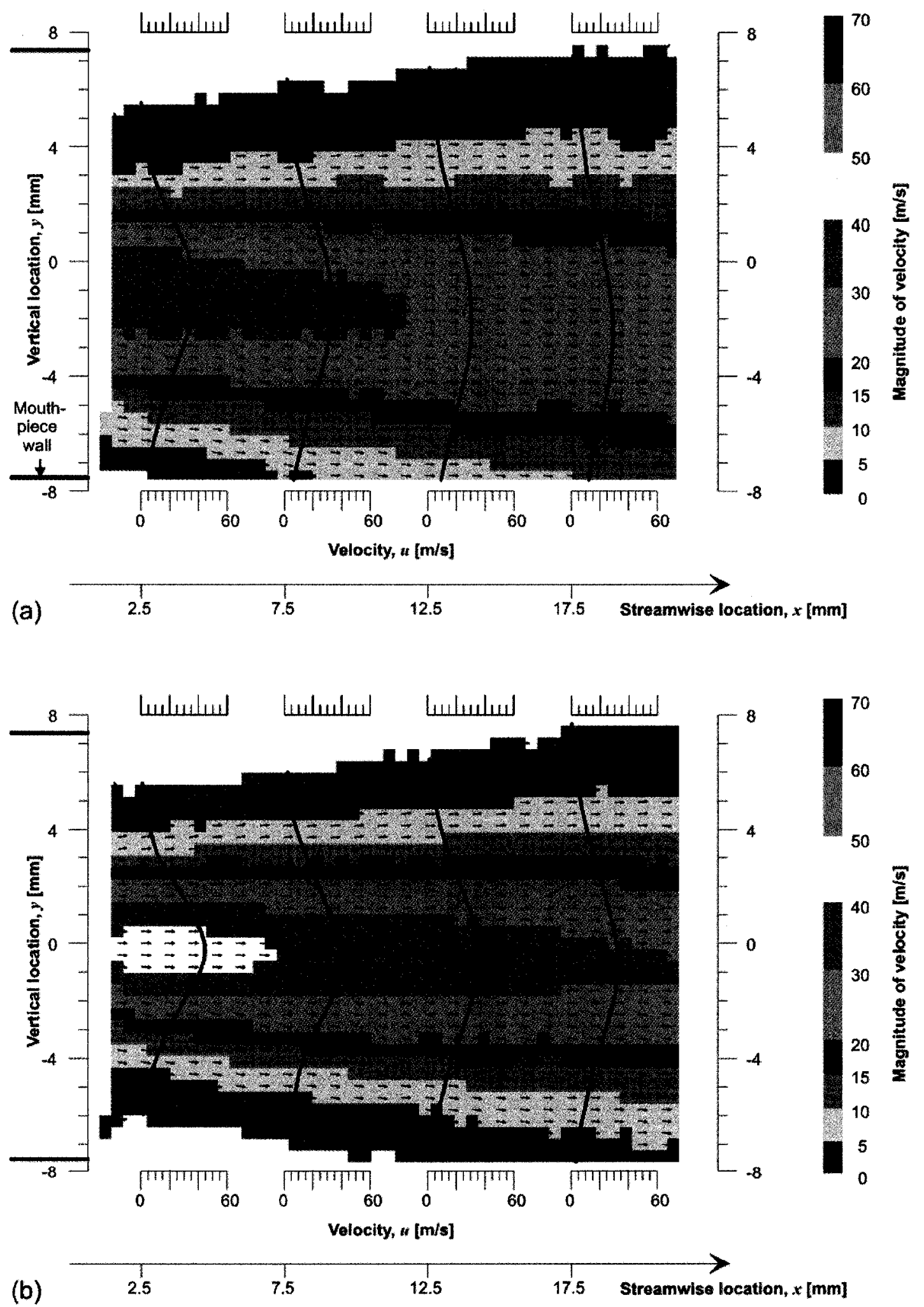

Figure 4.6 - Average vector velocity flow field. Ventolin, open air, (a) 20 and (b) $40 \mathrm{~ms}$. 

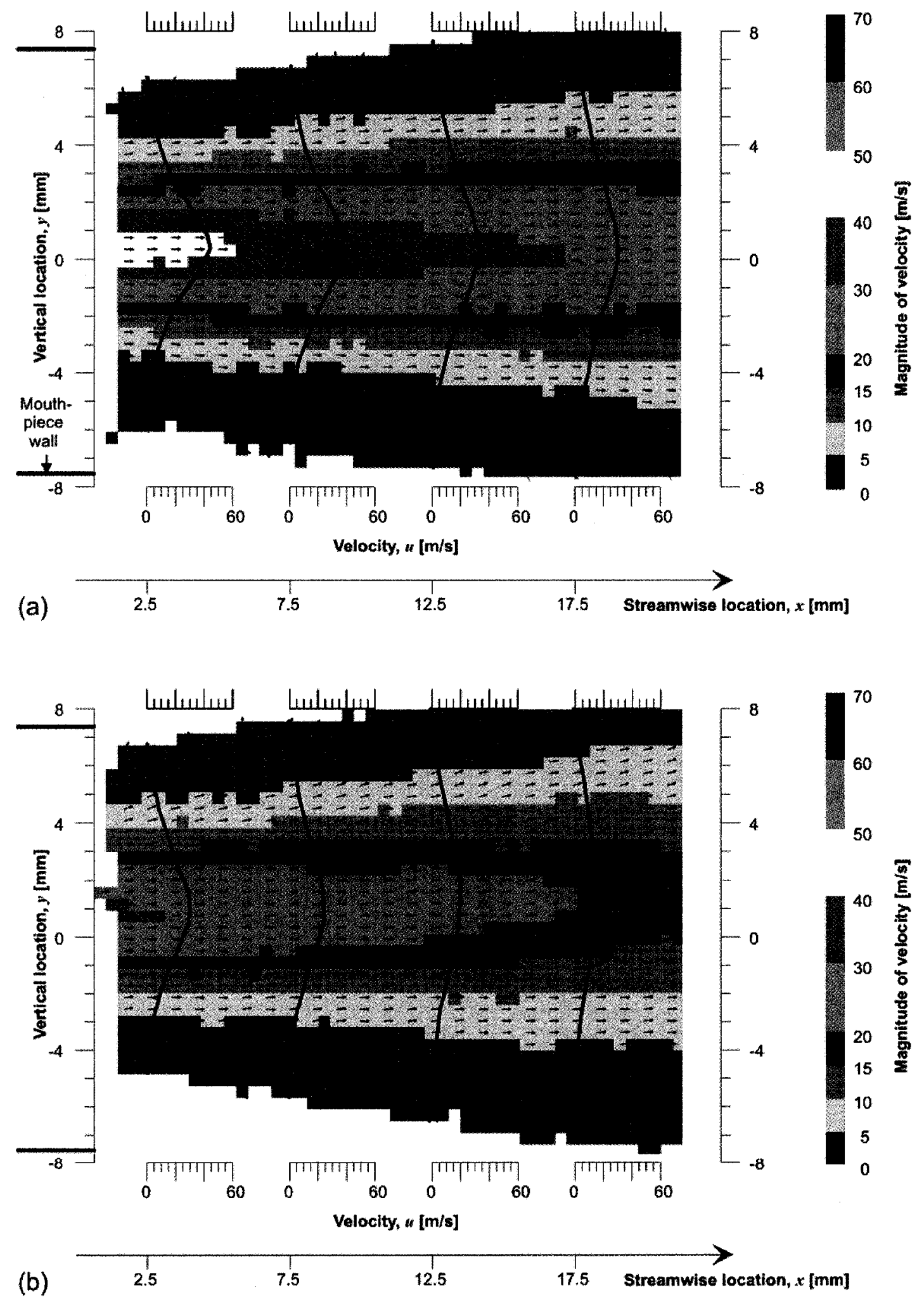

Figure 4.7 - Average vector velocity flow field. Ventolin, open air, (a) 60 and (b) $80 \mathrm{~ms}$. 


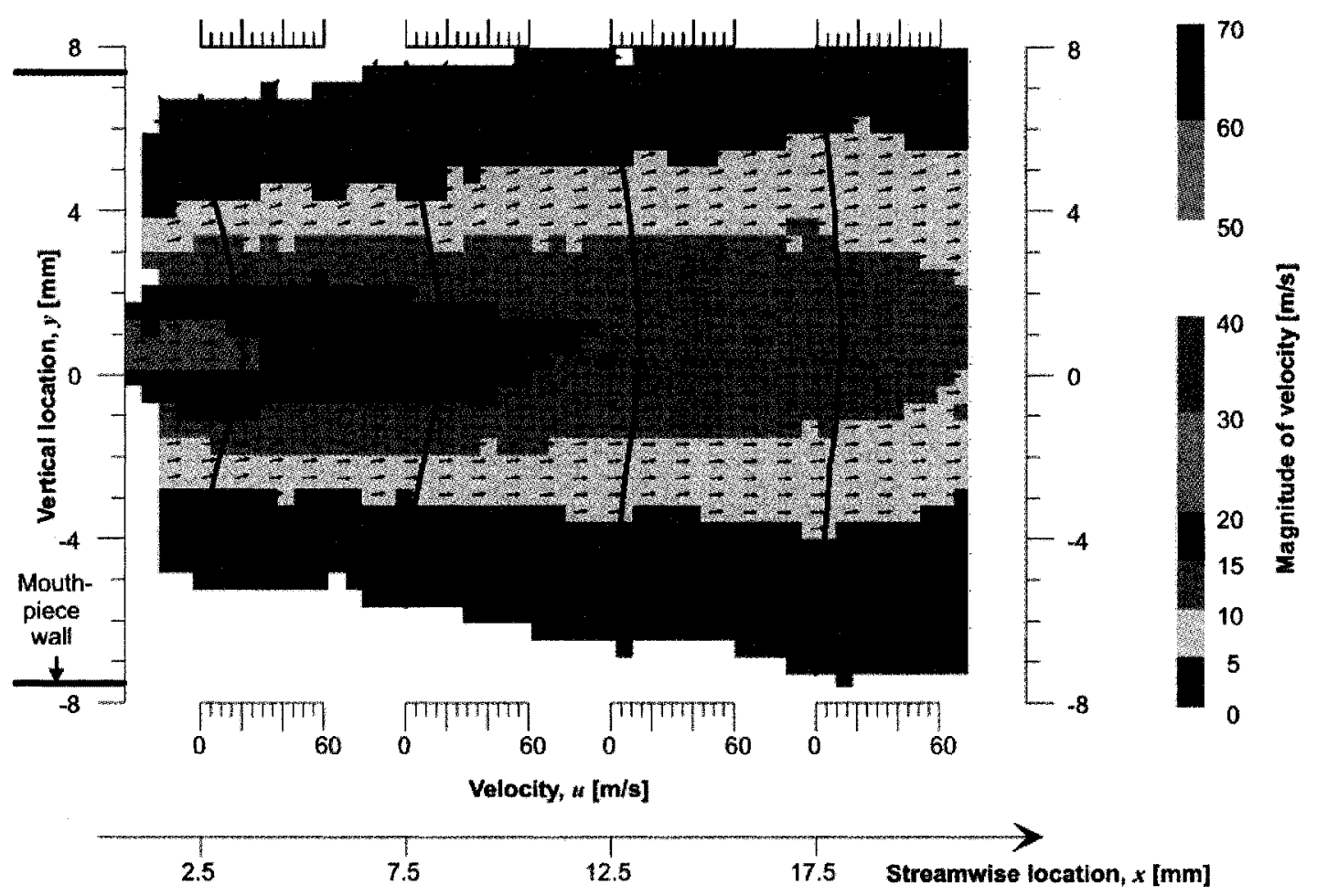

Figure 4.8 - Average vector velocity flow field. Ventolin, open air, $100 \mathrm{~ms}$.

By comparing the profiles at time delays of $2 \mathrm{~ms}, 10 \mathrm{~ms}$, and $40 \mathrm{~ms}$, two important observations can be made. First, the peak velocity does not decrease monotonically throughout the spray event (the peak velocity at $60 \mathrm{~ms}$ is significantly greater than that at $10 \mathrm{~ms}$ ). This will be discussed in detail in Section 5.1. Second, the vertical location of the peak velocity, which is directly related to the jet direction, does not remain constant over time. In fact, the peak in the streamwise velocity at $10 \mathrm{~ms}$ is about $4^{\circ}$ below horizontal $(x z)$, as will be discussed in detail in Section 5.2.

\subsection{Air Flow of 30 SLPM Only}

To allow proper comparison of spray geometry and velocities with and without a 30 SLPM co-flow of air, it is important to know what velocities are created by the air 
flow itself. As an initial estimate, if the velocity at the mouthpiece outlet is assumed to be uniform, and the mouthpiece area is measured to be $3.2 \mathrm{~cm}^{2}$ then incompressible mass conservation dictates that the streamwise velocity will be about $1.6 \mathrm{~m} / \mathrm{s}$. The Reynolds number of the air flow based on this velocity and the hydraulic diameter of the mouthpiece opening is:

$$
\operatorname{Re}=\frac{\rho V D_{h}}{v}=\frac{\left(1.2 \mathrm{~kg} / \mathrm{m}^{3}\right)(1.6 \mathrm{~m} / \mathrm{s})(1.7 \mathrm{~m})}{1.5 \cdot 10^{-5} \mathrm{~kg} / \mathrm{m} \cdot \mathrm{s}}=2200
$$

indicating that the air flow is most likely turbulent as it leaves the mouthpiece. This was confirmed by examination of instantaneous images of the air flow.

In reality, the flow at the mouthpiece outlet will not be uniform. It was expected that the $90^{\circ}$ bend in the pMDI casing would result in greater velocities near the bottom of the mouthpiece outlet plane since fluid from the inside of the bend will be unable to properly navigate the sharp bend of the inner edge. The experimental results agreed with this prediction, as shown in Figure 4.9. (Note that the lower velocities involved necessitated the use of a different colour scale than was used previously.) The streamwise velocity near the top of the mouthpiece outlet plane is less than $1 \mathrm{~m} / \mathrm{s}$, whereas the streamwise velocity near the bottom reaches a maximum value of $4.18 \mathrm{~m} / \mathrm{s}$.

It is also possible that the flow around the bend will create Dean vortices at the outlet plane. Dean vortices occur in flows around bends where the flow from the inner wall of the bend overshoots the bend and ends up on the outer wall. This then creates a pair of counter-rotating Dean vortices. In the flow of air through a pMDI, these vortices (if they 
exist) will induce downward velocities along the centreline of the flow, and upward velocities at the edges of the mouthpiece, as shown in Figure 4.10.

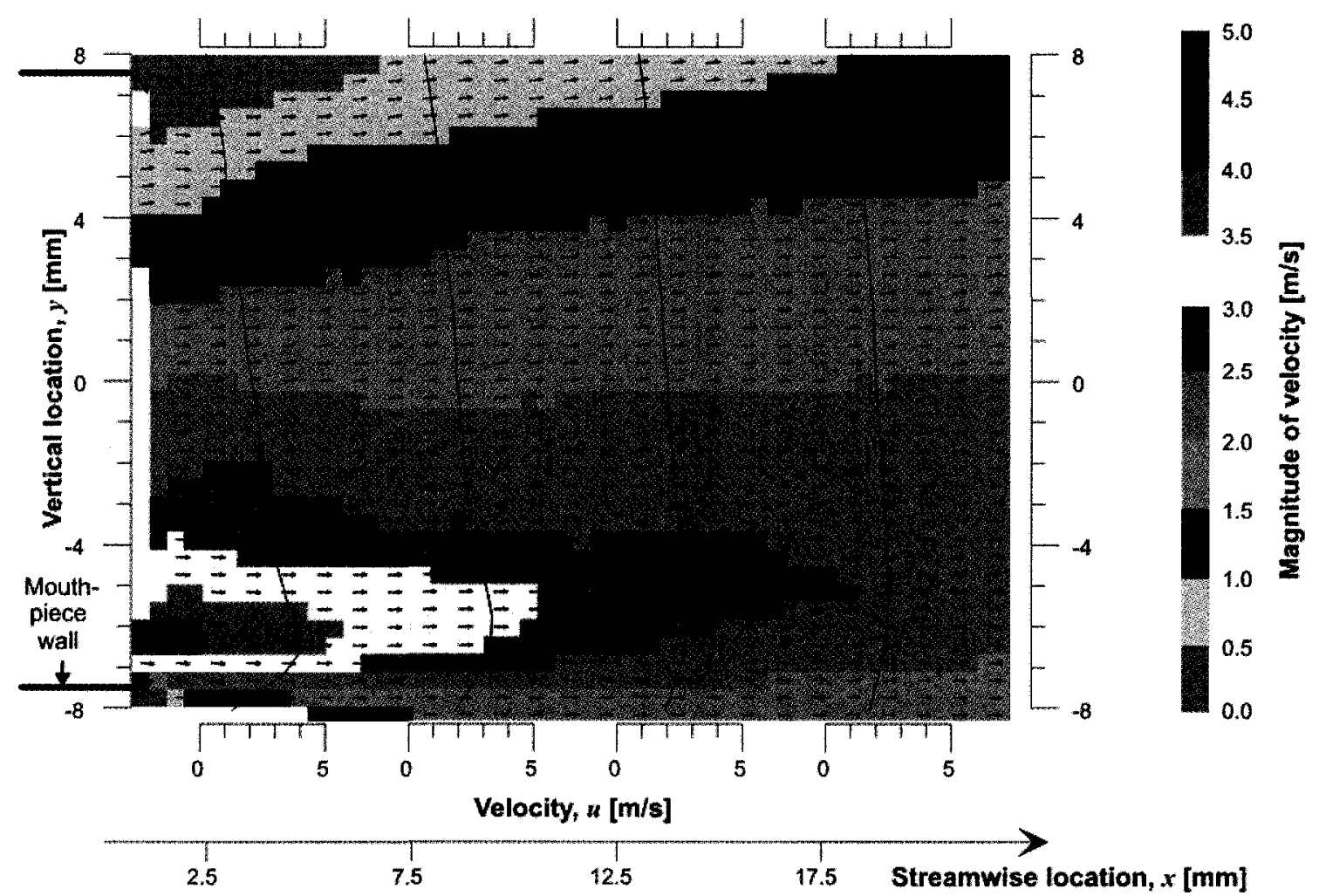

Figure 4.9 - Velocity profiles of 30 SLPM of air.

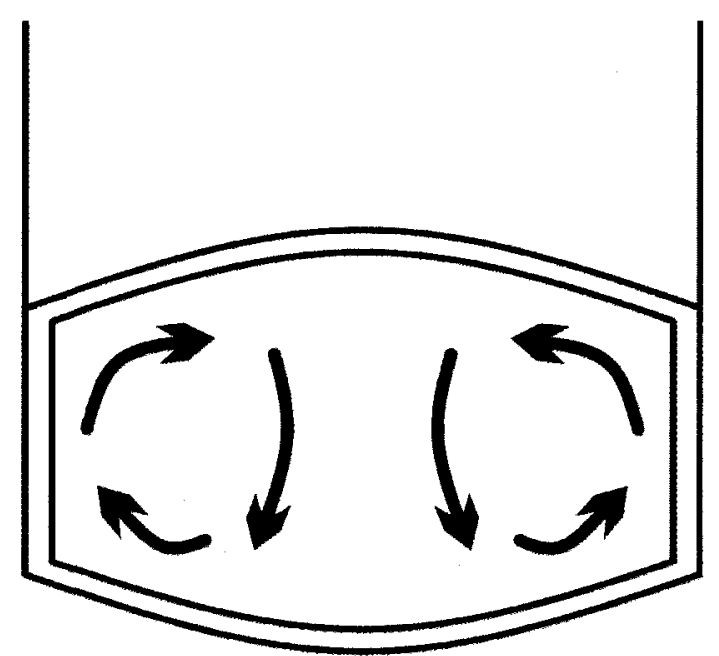

Figure 4.10 - Direction of Dean vortices, if present. View into mouthpiece. 


\subsection{Spray Issued Into Open Air with 30 SLPM Air Flow}

To determine whether the addition of the secondary air flow will or will not have a statistically significant effect on the spray velocity, 1000 PIV image pairs were collected of the pMDI issuing into open air along with the secondary air flow of 30 SLPM. Figures $4.11-4.15$ compare vertical profiles of streamwise velocity with and without the secondary air flow at selected streamwise locations, clearly showing the magnitude and locations of the differences between the two flows.

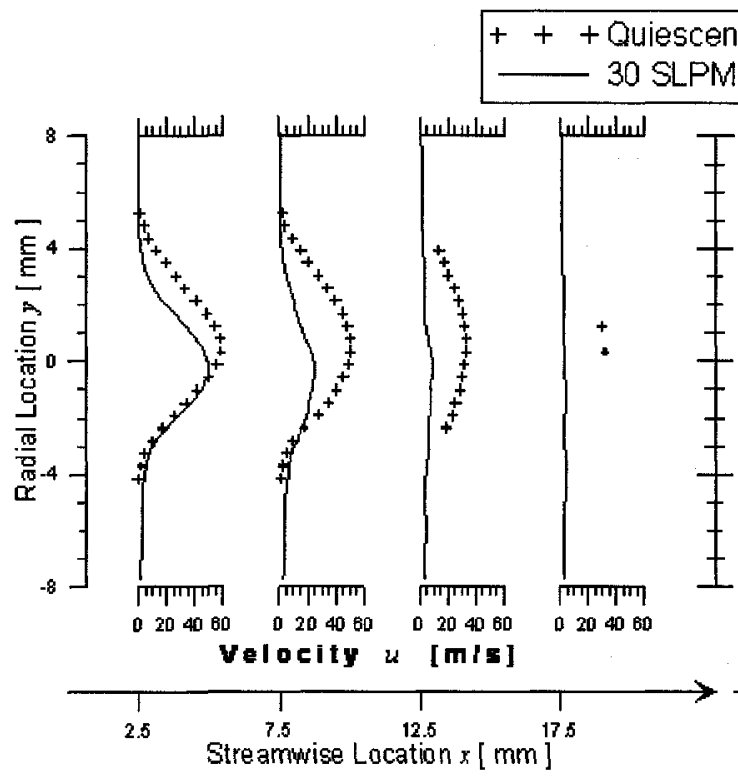

(a)

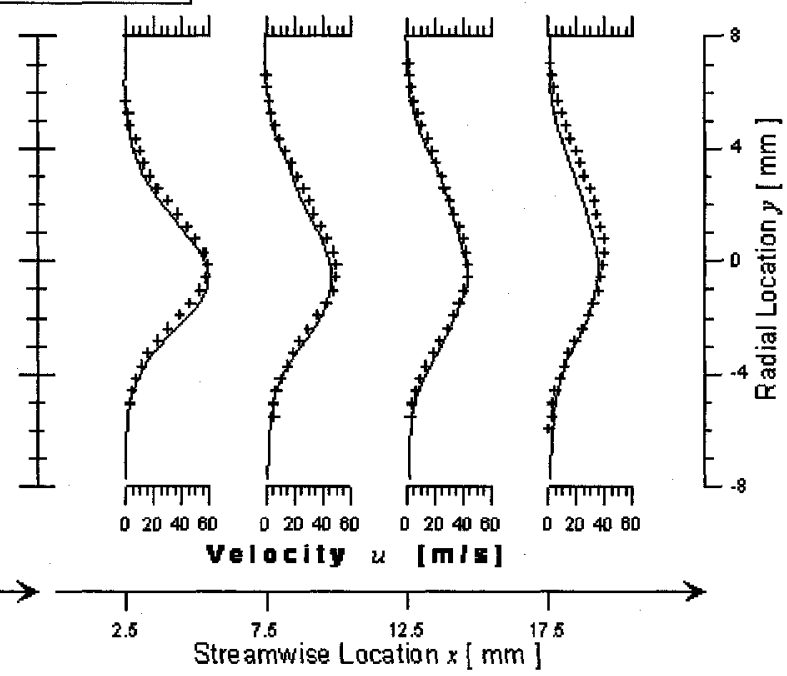

(b)

Figure 4.11 - Comparison of vertical profiles of streamwise spray velocity with and without a 30 SLPM air flow. Ventolin, (a) 1.3 and (b) 2 ms. 


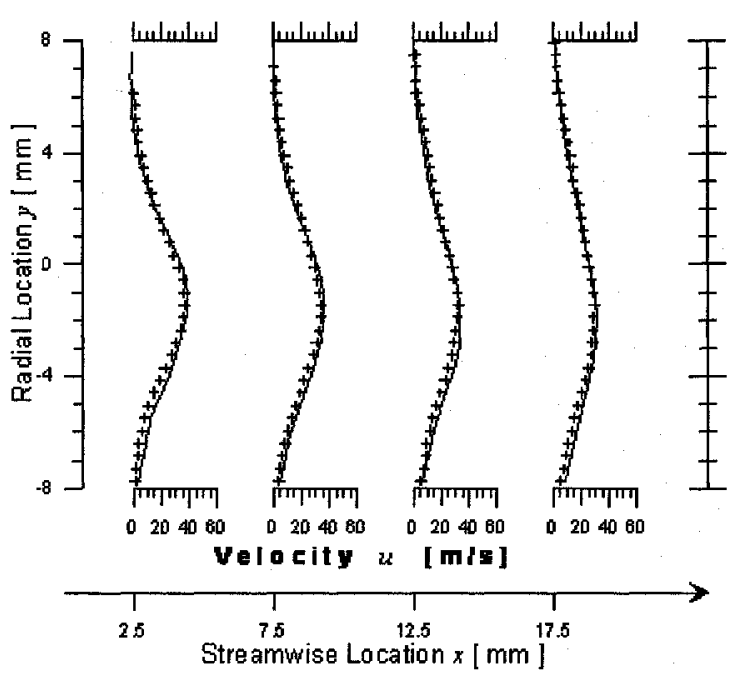

(a)

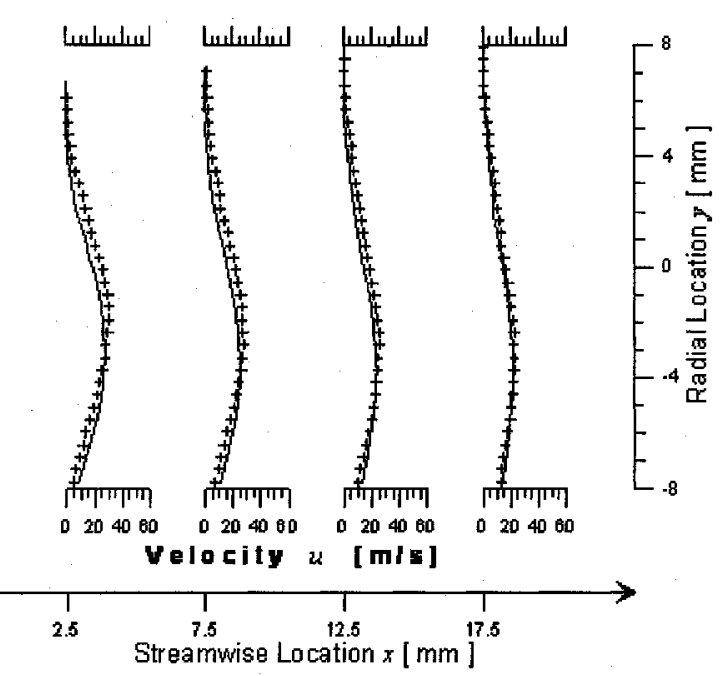

(b)

Figure 4.12 - Comparison of vertical profiles of streamwise spray velocity with and without a 30 SLPM air flow. Ventolin, (a) 5 and (b) 10 ms.

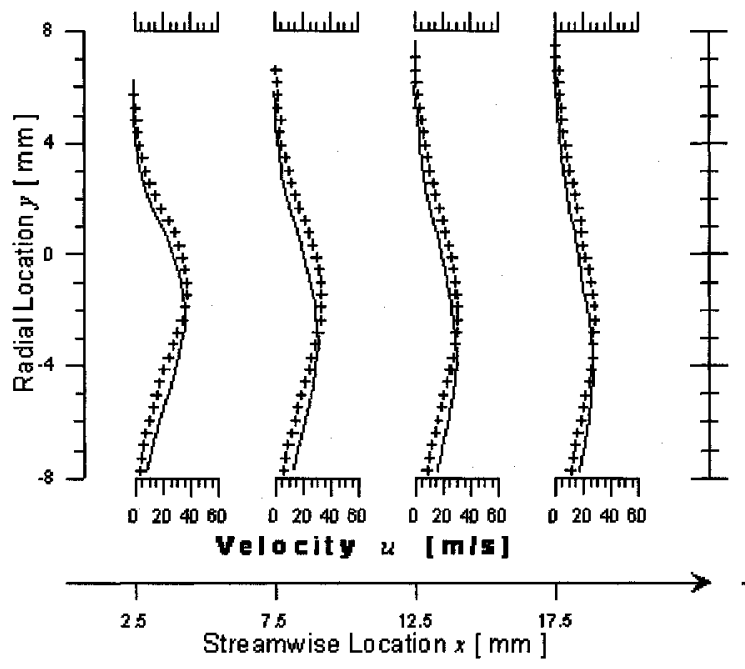

(a)

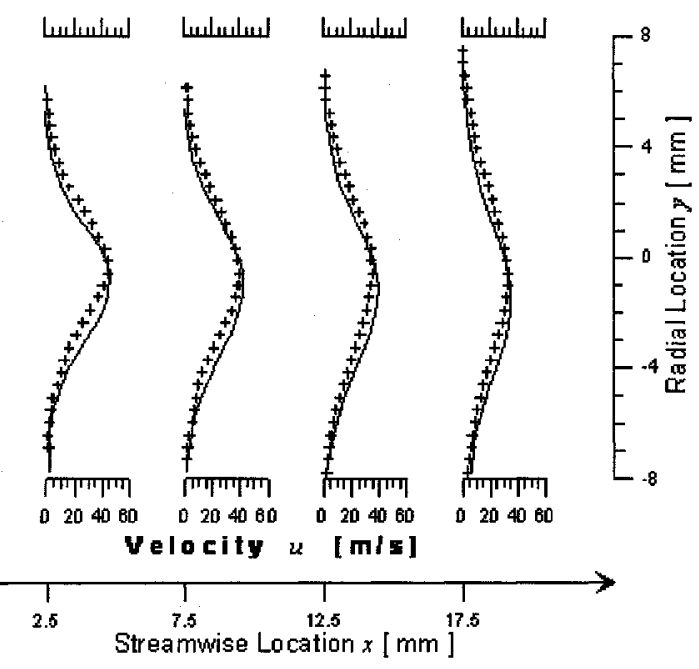

(b)

Figure 4.13 - Comparison of vertical profiles of streamwise spray velocity with and without a 30 SLPM air flow. Ventolin, (a) 20 and (b) 40 ms. 


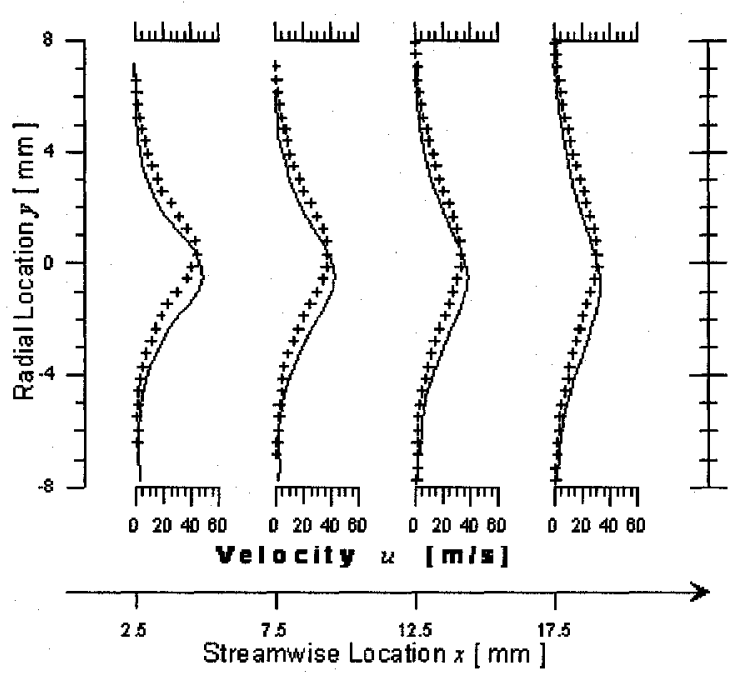

(a)

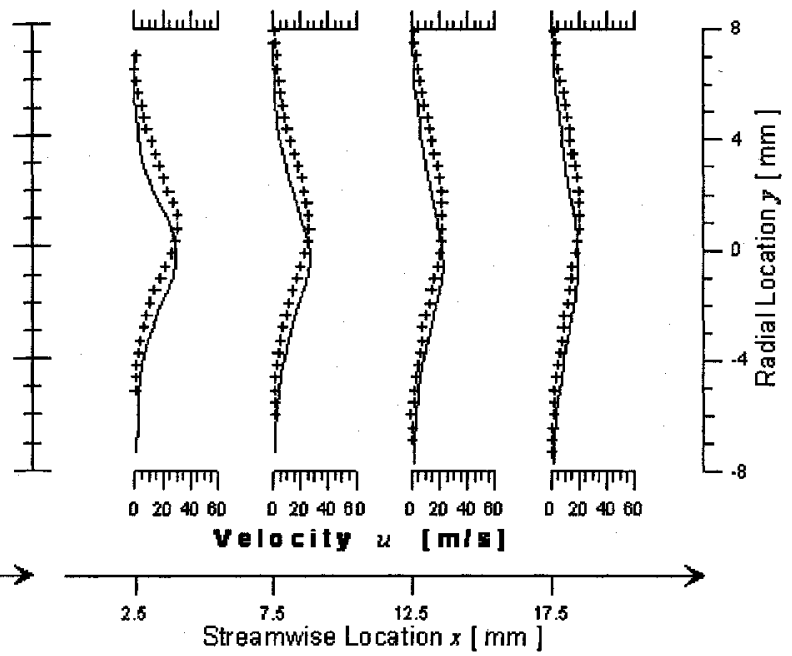

(b)

Figure 4.14-Comparison of vertical profiles of streamwise spray velocity with and without a 30 SLPM air flow. Ventolin, (a) 60 and (b) 80 ms.

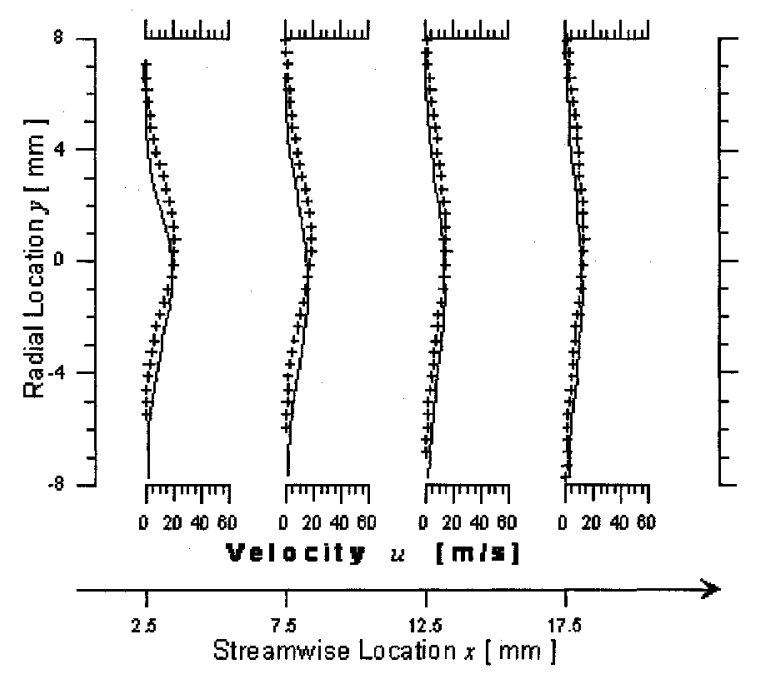

Figure 4.15 - Comparison of vertical profiles of streamwise spray velocity with and without a 30 SLPM air flow. Ventolin, $100 \mathrm{~ms}$.

The most obvious difference is seen in the earliest time delay of $1.3 \mathrm{~ms}$. This seemingly large difference in peak velocity is due to slight differences in the spray penetration. Outside of the spray the lack of particles in the quiescent air case will yield a cross-correlation based solely on noise,,yielding no vector result. Conversely, in the case 
with a secondary air flow, seeded air is present beyond the spray tip, and is presumably moving at a slower velocity, closer to that of the secondary air flow velocity field presented in Section 4.3. Thus the average velocity in the near-tip region for the case with a secondary air flow is biased toward lower velocity values.

Another important difference between the cases with and without the secondary air flow is the shift in the location of the peak streamwise velocity seen after the addition of the co-flow. This is a result of a change in spray direction, which will be discussed in Section 5.2.

\subsection{Spray Issued Into Add-On Device with 30 SLPM Air Flow}

The addition of an add-on device was not expected to alter the flow at the pMDI mouthpiece. To test this hypothesis, measurements were taken inside the add-on device at the same field of view as previous measurements. As well, a second set of measurements was taken at the outlet of the add-on device, where the user normally inhales. Here the effect of the device is clearly seen, with velocities much lower than measured at the pMDI mouthpiece.

\subsubsection{Spray Velocities Inside the Add-on Device}

Spray velocity measurements taken inside the add-on device were in fact very similar to those taken without it. The vector velocity flow field overlaid with four vertical profiles of streamwise velocity at the ten time delays selected are presented in Figures 4.16 4.20. 
Visual inspection indicates that the addition of the add-on device has a smaller effect on the pMDI spray velocity than was seen when comparing the quiescent and co-flow cases. Once again the peak velocity has been shifted downward due to a change in the spray direction, though the shift is smaller and not present at all time delays. Thus the presence of the add-on device (or, conceivably, of the oral cavity of a patient using pMDI alone), has little to no effect on the velocity field in the region studied $(\sim 20 \mathrm{~mm} \times 16 \mathrm{~mm}$ at the pMDI outlet).

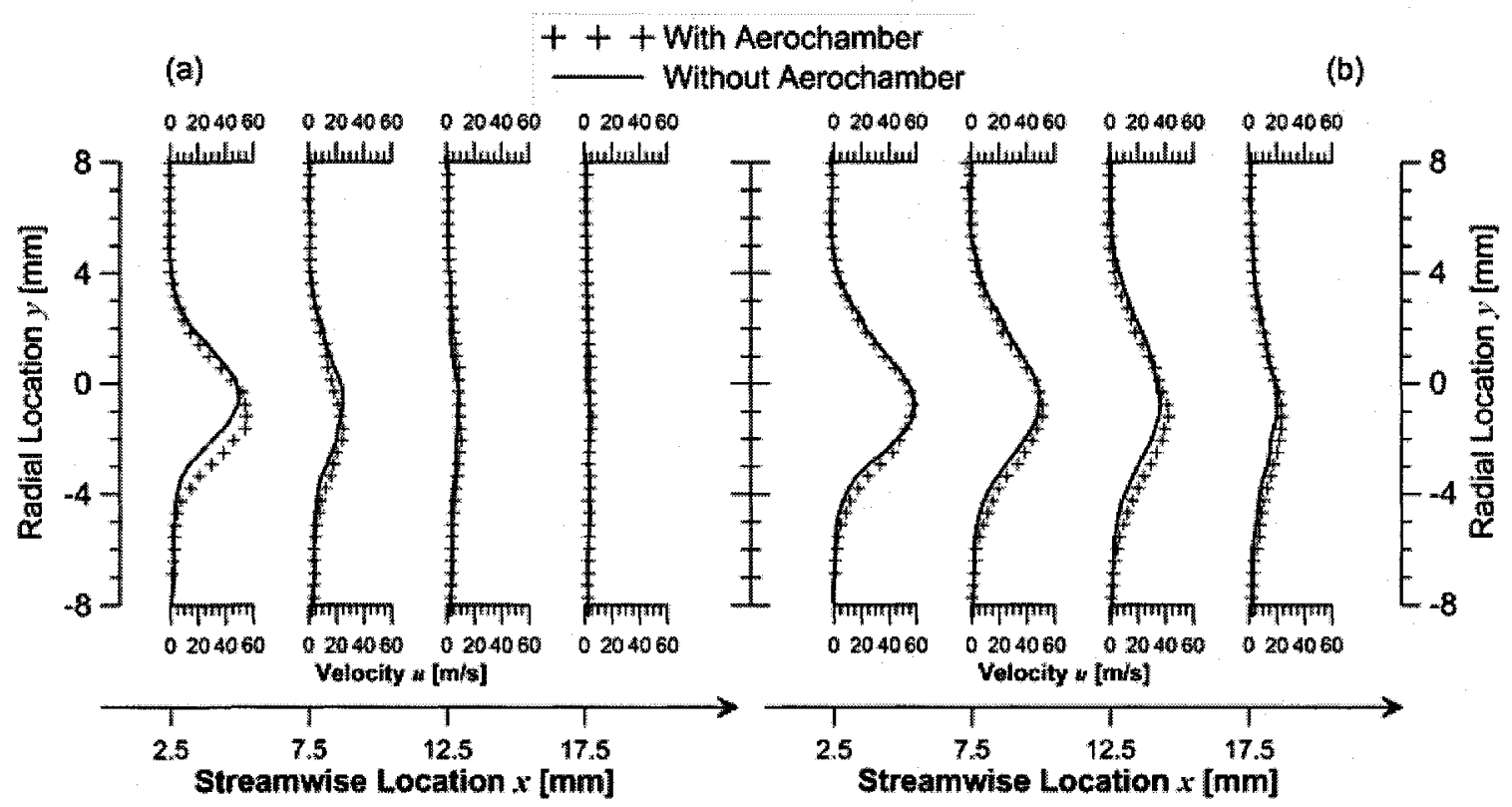

Figure 4.16-Vertical profiles of streamwise spray velocity with and without the Aerochamber. Ventolin, (a) 1.3 and (b) $1.7 \mathrm{~ms}$. 


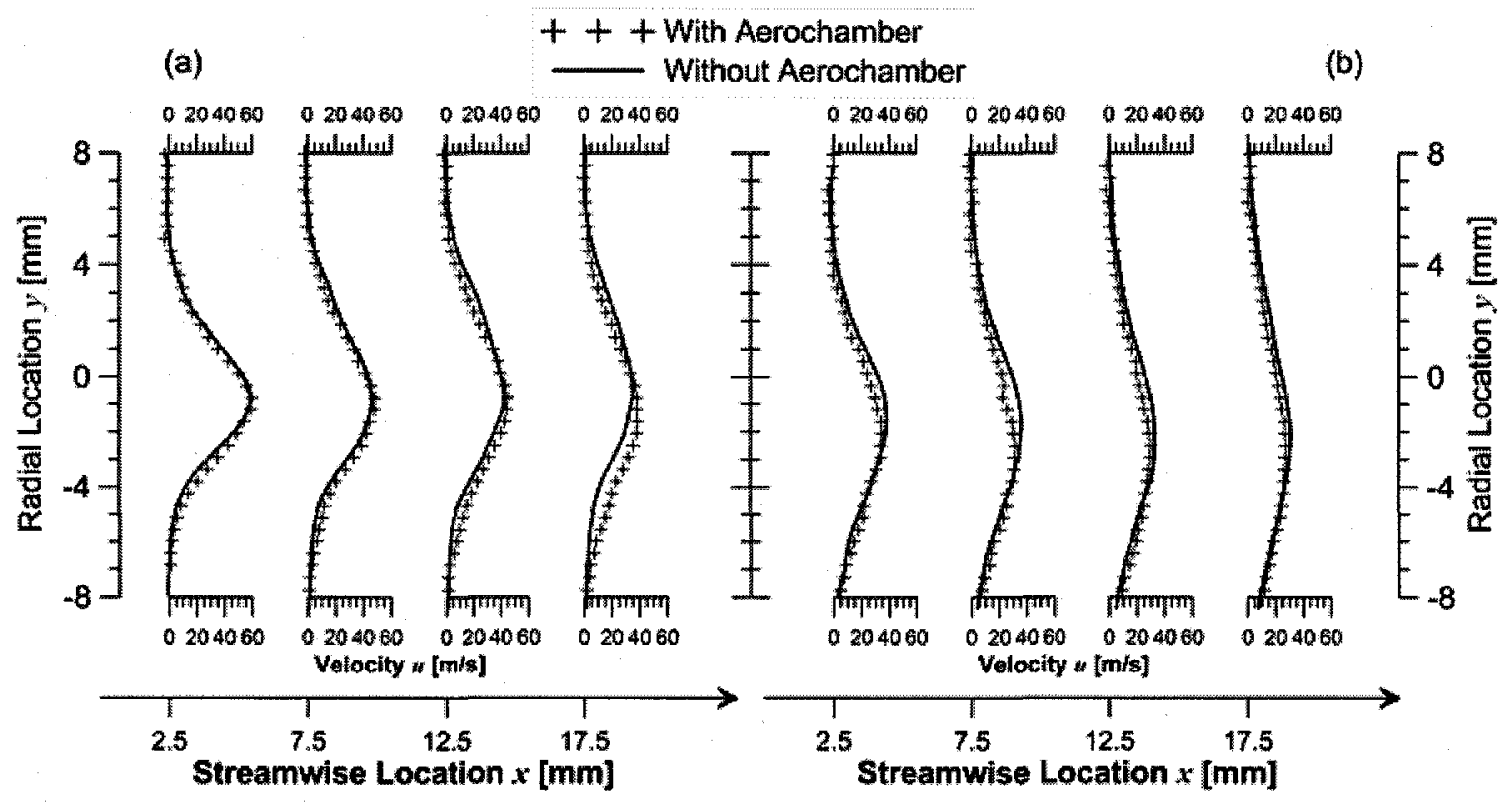

Figure 4.17 - Vertical profiles of streamwise spray velocity with and without the

Aerochamber. Ventolin, (a) 2 and (b) 5 ms.

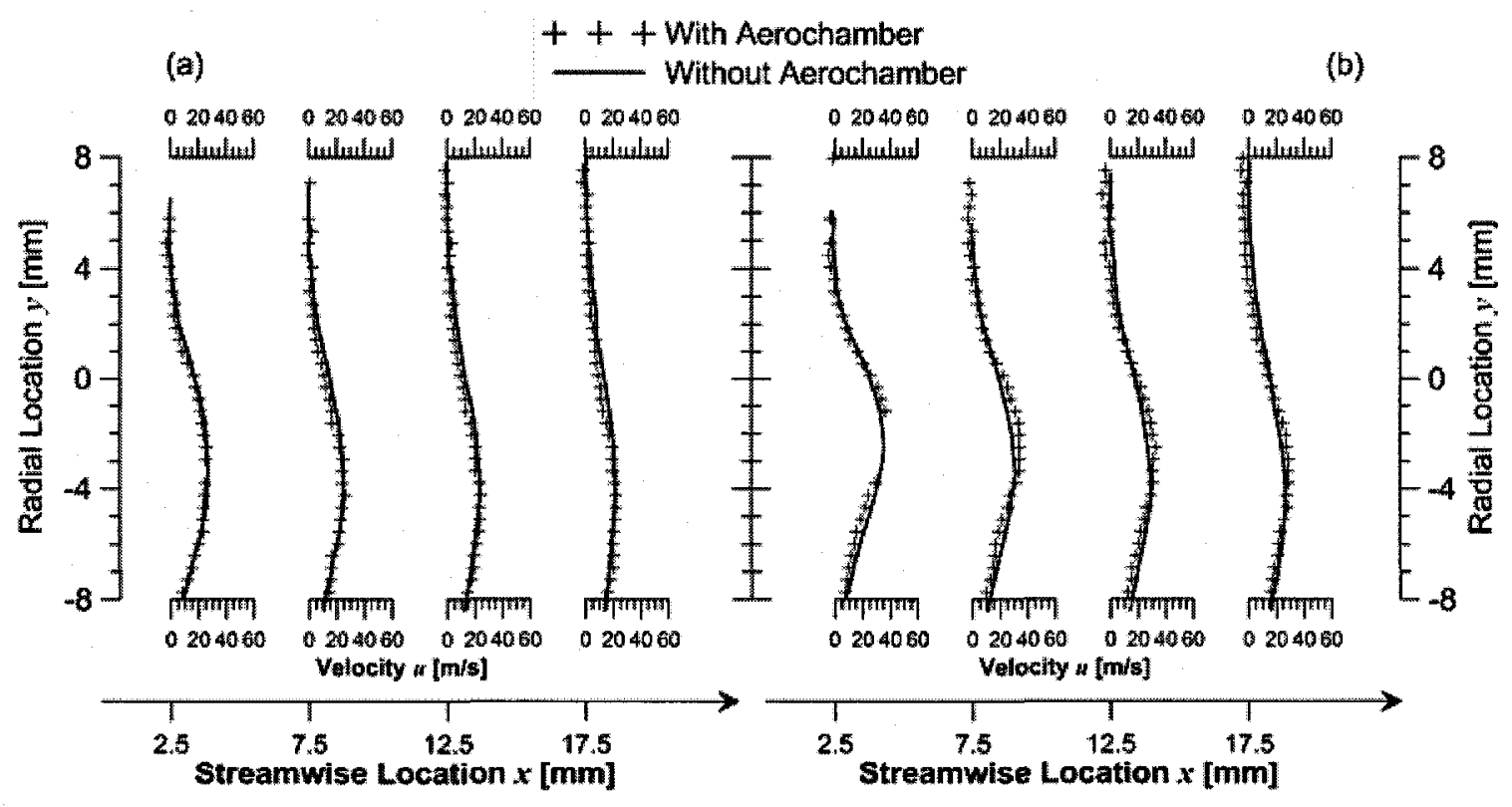

Figure 4.18 - Vertical profiles of streamwise spray velocity with and without the Aerochamber. Ventolin, (a) 10 and (b) $20 \mathrm{~ms}$. 


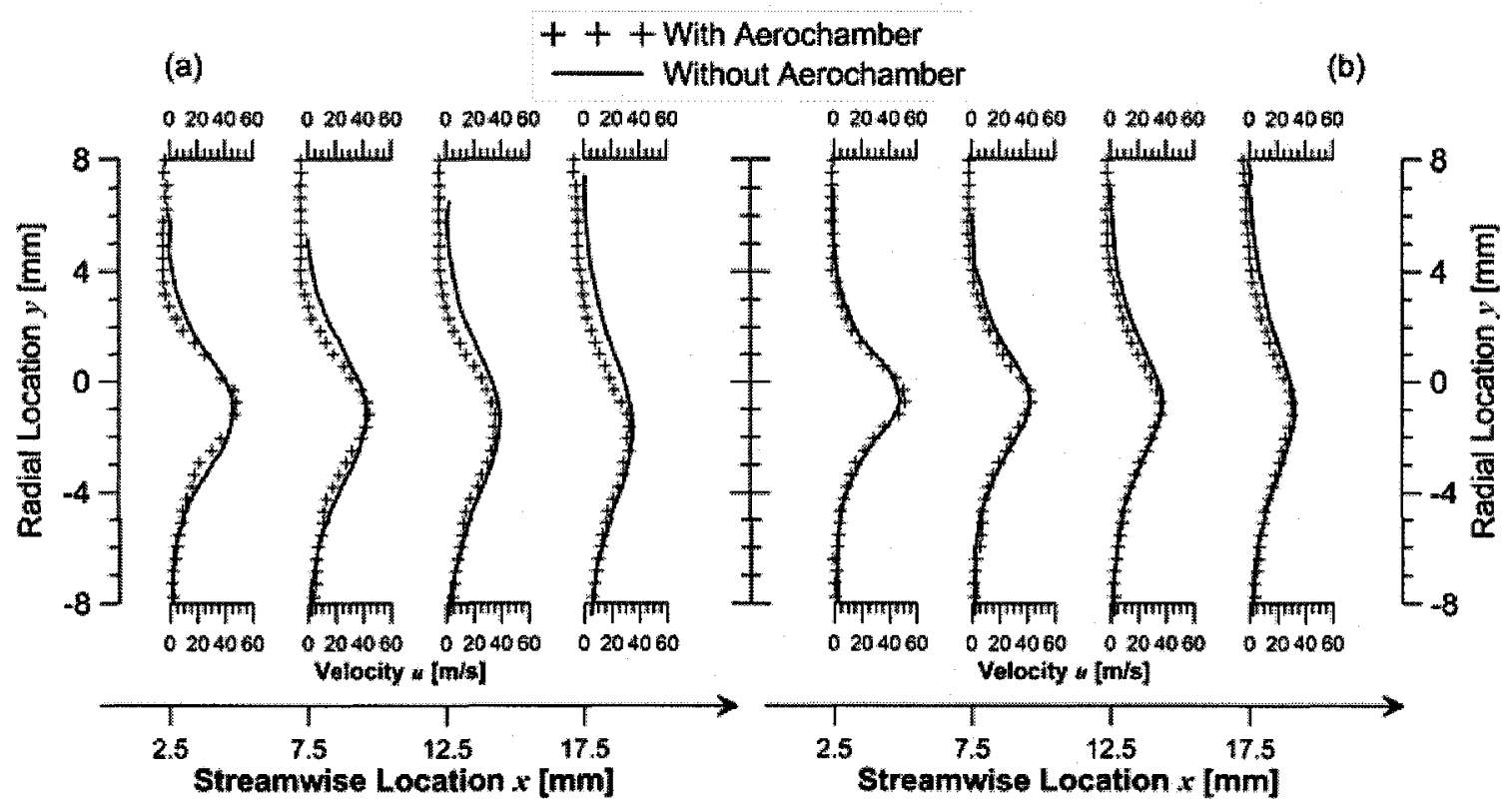

Figure 4.19-Vertical profiles of streamwise spray velocity with and without the Aerochamber. Ventolin, (a) 40 and (b) $60 \mathrm{~ms}$.

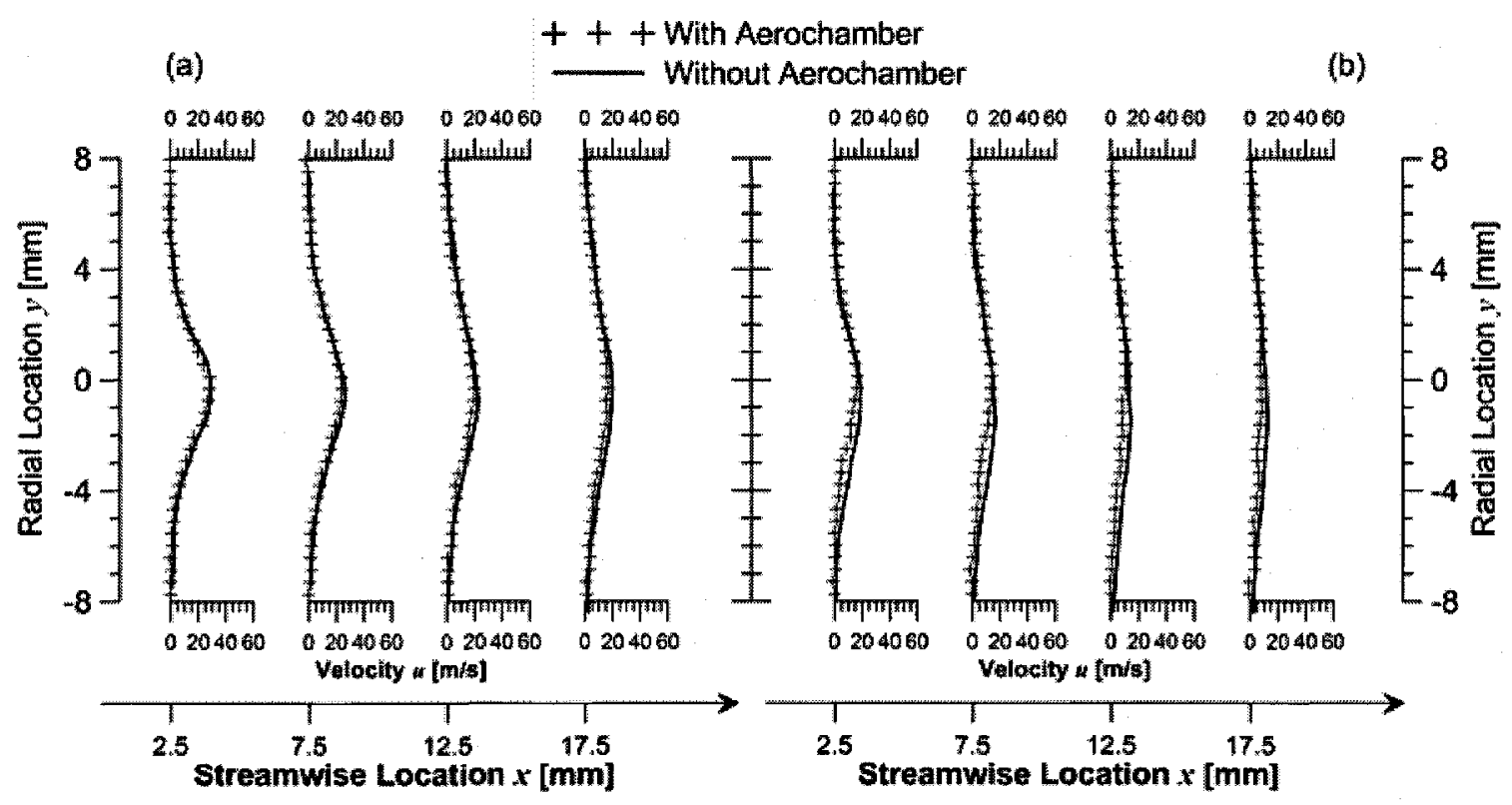

Figure 4.20-Vertical profiles of streamwise spray velocity with and without the Aerochamber. Ventolin, (a) 80 and (b) 100 ms. 


\subsubsection{At the Outlet of the Add-On Device}

Measurements were taken at the outlet of the add-on device at various time delays (see Table 3.7) after actuation. The same measurement region of $20 \mathrm{~mm}$ in the streamwise direction by $16 \mathrm{~mm}$ in the vertical direction, centred vertically on the mouthpiece, was used. The velocities measured were smaller than those at the pMDI outlet, thus providing an entirely different inlet flow to a patient using such a device. Unfortunately, the trend in the results is very different from those observed at the pMDI outlet, including multiple peaks in time and asymmetry about the horizontal $(x z)$ plane. Vertical profiles of streamwise velocity are included in Appendix D.

A possible explanation for this behaviour is that the valve at the spacer outlet may not have remained open for the duration of the spray event. This valve is a thin, flexible rubber flap which opens due to the pressure gradient created by inhalation. Although the valve state was verified before every set of ten actuations, it is possible that it became stuck in the closed position during subsequent actuations. 


\section{Chapter 5: Analysis and Discussion}

\subsection{Temporal Analysis of the pMDI Spray}

To better visualize the temporal evolution of the spray, the streamwise velocity has been extracted from the vector flow field at three points in the streamwise direction, vertically centred at the height of the pMDI nozzle. These velocities are presented in Figure 5.1 along with similar data presented by Dunbar (1996).

The bimodal trend in centreline velocity mentioned earlier is clearly apparent. In the present data, the initial velocity spike lasts approximately $5 \mathrm{~ms}$ followed by a second broad peak spanning $70 \mathrm{~ms}$. This representation alone, combined with the knowledge that the vertical location of the peak velocity changes in time, might lead one to believe that the appearance of the second maximum is simply due to a change in peak location. A plot similar to Figure 5.1, modified to show the magnitude of the peak streamwise velocity (wherever that peak is located) at a given streamwise location, is presented in Figure 5.2. It now becomes clear that the two maxima do in fact exist, regardless of the spatial shift of the streamwise velocity peak.

Based on the numerical models of Fletcher (1975) and Clark (1991), Wigley et al. (2002) suggest that the two maxima occur primarily due to a mass-flow imbalance between the flow into and out of the pMDI expansion chamber early in the event. They 
suggest that this causes the pressure in the expansion chamber to rise, decreasing the vapour mass fraction and thus decreasing the local speed of sound. This chokes the flow out of the nozzle orifice and brings the velocity to a minimum. The increased pressure in the expansion chamber also causes a reduction in the mass flow rate into the expansion chamber, allowing the outflow to once again accelerate to a second maximum. After this point, the velocity decreases monotonically until the end of the event.

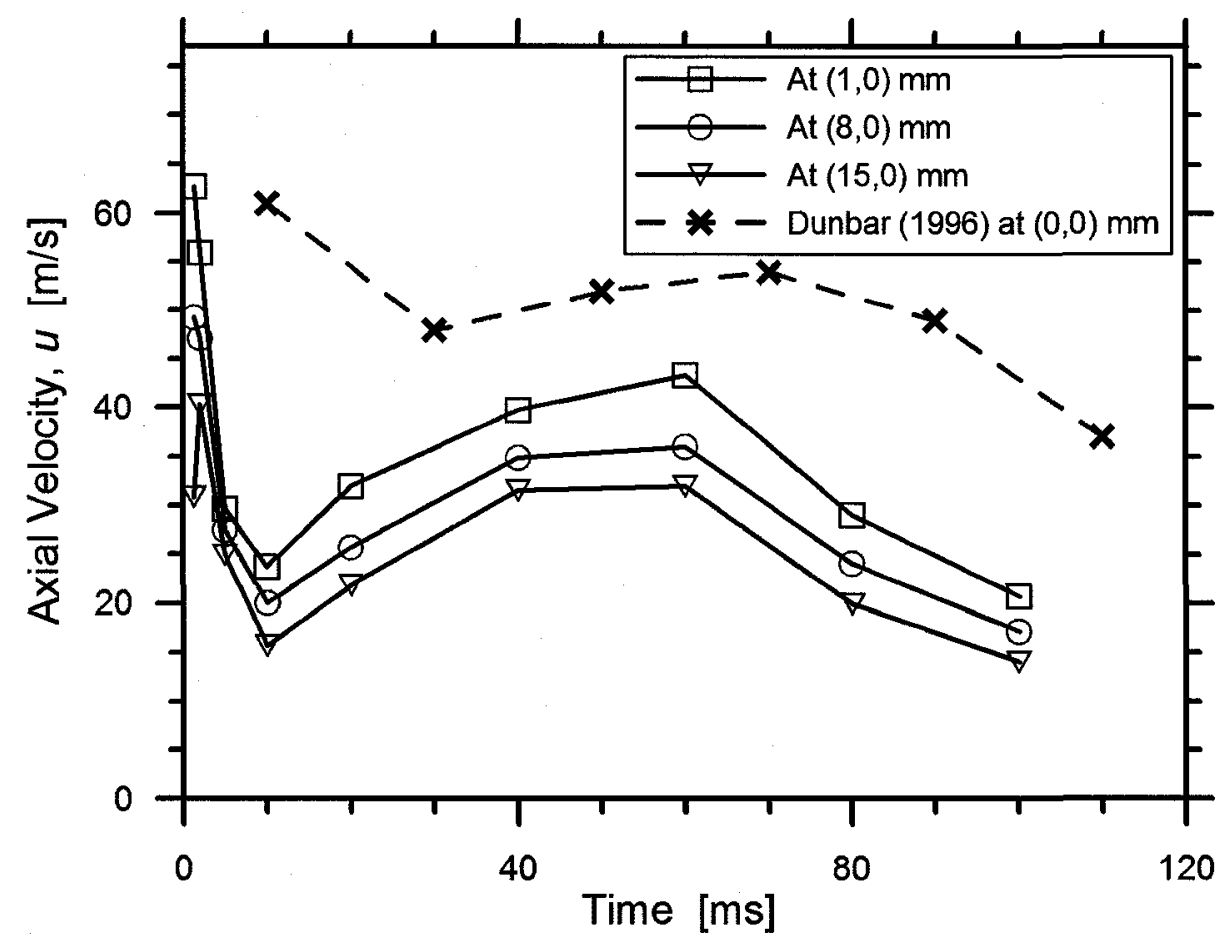

Figure 5.1 - Temporal evolution of the average streamwise velocity during the first $120 \mathrm{~ms}$ of the spray event. Ventolin only. 


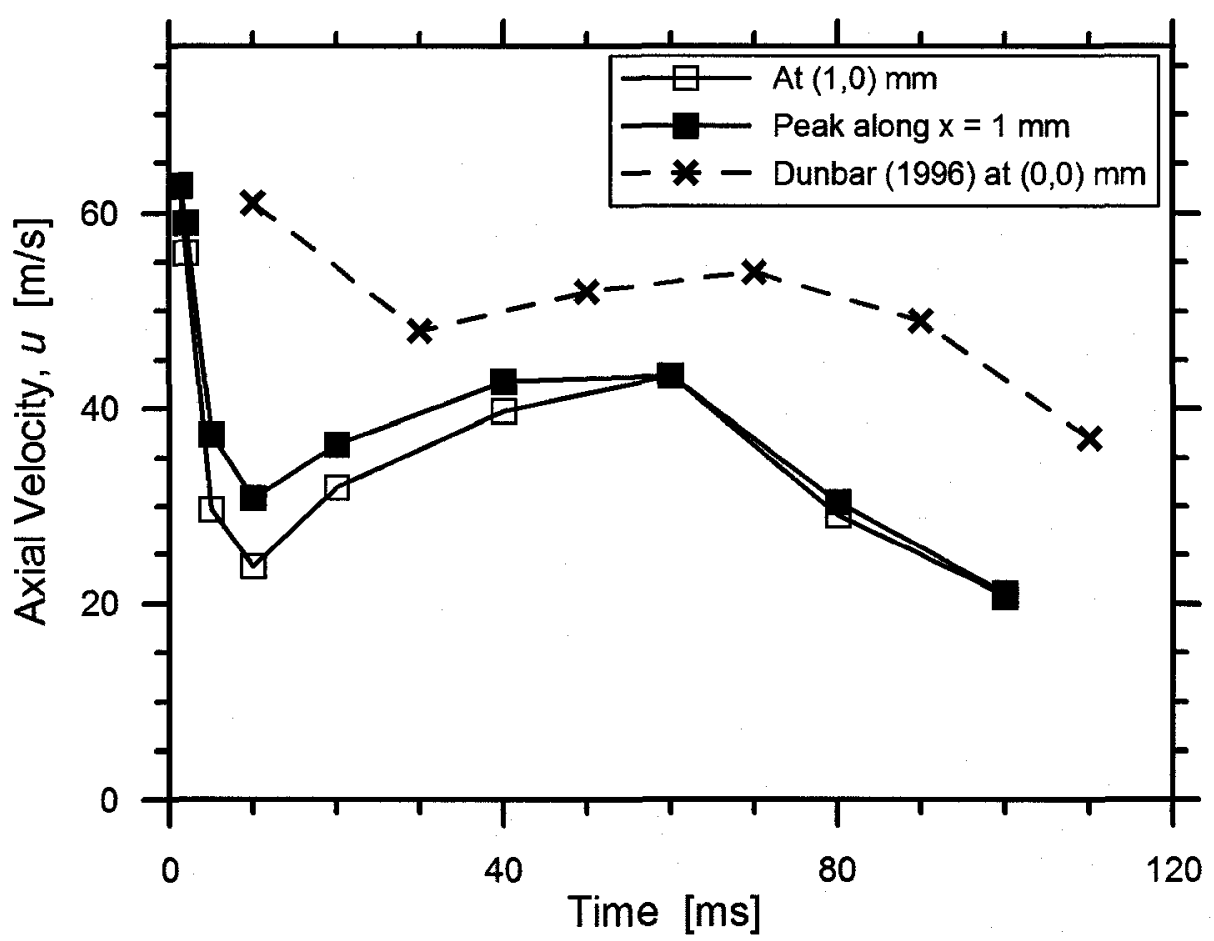

Figure 5.2 - Temporal evolution of the average streamwise velocity during the first $120 \mathrm{~ms}$ of the spray event. Comparing the peak velocity for a given streamwise location with the velocity on the centreline. Ventolin only.

\subsection{Analysis of Geometric Characteristics of pMDI Spray}

To characterize both the spray cone angle and the mean spray direction, both of the methods described in Section 3.9 were employed. Though the trends shown using both methods are similar, the magnitudes of the results are slightly different. Figure 5.3 compares the temporal evolution of the spray direction using a ratiopharm-brand canister calculated using both methods. Also shown are the results using the velocity-based method for a ratiopharm-brand canister actuated within the 30 SLPM co-flow. 


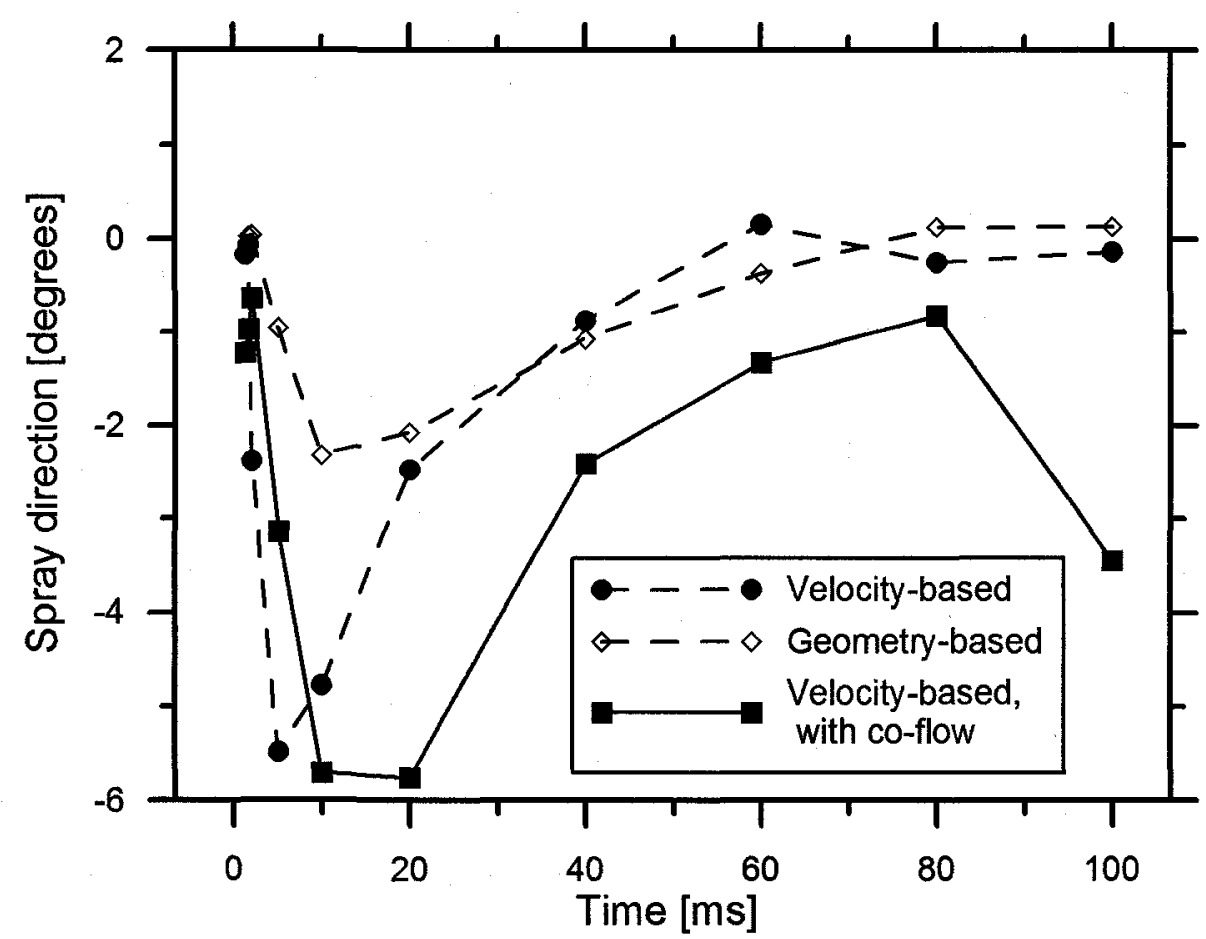

Figure 5.3 - Comparison of the spray direction calculated using both calculation methods, as well as the effect of the 30 SLPM co-flow.

The two calculation methods produce similar trends, though of different magnitudes. This is possibly due to the selection of a noise threshold for the geometry-based calculations. Though a basic sensitivity analysis was performed, the specification of the threshold was nonetheless a user-specified quantity which has a direct influence on the results. The geometry-based method was rendered meaningless by the presence of the seeding particles during trials with the co-flow, and so is not presented. Concentrating on only the velocity-based measurements, there are two significant differences between the quiescent air and the co-flow spray direction measurements in Figure 5.3. The first is that the addition of the co-flow pushes the spray direction downward for most measurement times. This is in agreement with the notion that the results from the spray 
in the co-flow should be somewhat similar to the addition of the spray field in quiescent air and the co-flow alone. Though it was never expected that the resultant would be equal to the sum of the two flow fields, it is reasonable that the addition of the co-flow would have a greater effect in areas where it created stronger velocities on its own. Another factor that likely contributes to the downward deflection is the probable presence of Dean vortices in the co-flow, as discussed in Section 4.3.

The other noticeable difference is the large downward deflection of the spray at $100 \mathrm{~ms}$. This is believed to be a non-physical result of the calculation method. Toward the end of the spray event $(\sim 100 \mathrm{~ms})$ the particle profile flattens as the peak and halfmaximum velocities also decrease to the same order of magnitude as the co-flow velocity. While the direction of the spray alone approaches 0 , the direction of the spray and co-flow will approach the direction of the co-flow. Though the spray direction of the co-flow cannot be defined (since it does not originate from a single point), it seems from Figure 4.9 that it would be a negative value. In this way the co-flow has a strong effect on both spray direction and cone angle at the end of the spray event.

Similarly, Figure 5.4 shows the results of the spray cone angle calculations using both methods for a pMDI actuated into quiescent air, as well as the FWHM criterion calculations for a pMDI actuated within a 30 SLPM co-flow of air. The minimum, maximum, and time-averaged spray directions and cone angles are presented in Table 5.1. Once again the two calculation methods yield results with similar trends. The spray cone angle can also be seen to increase drastically at the last time delay, for the same reasons the spray direction deflected downward. 


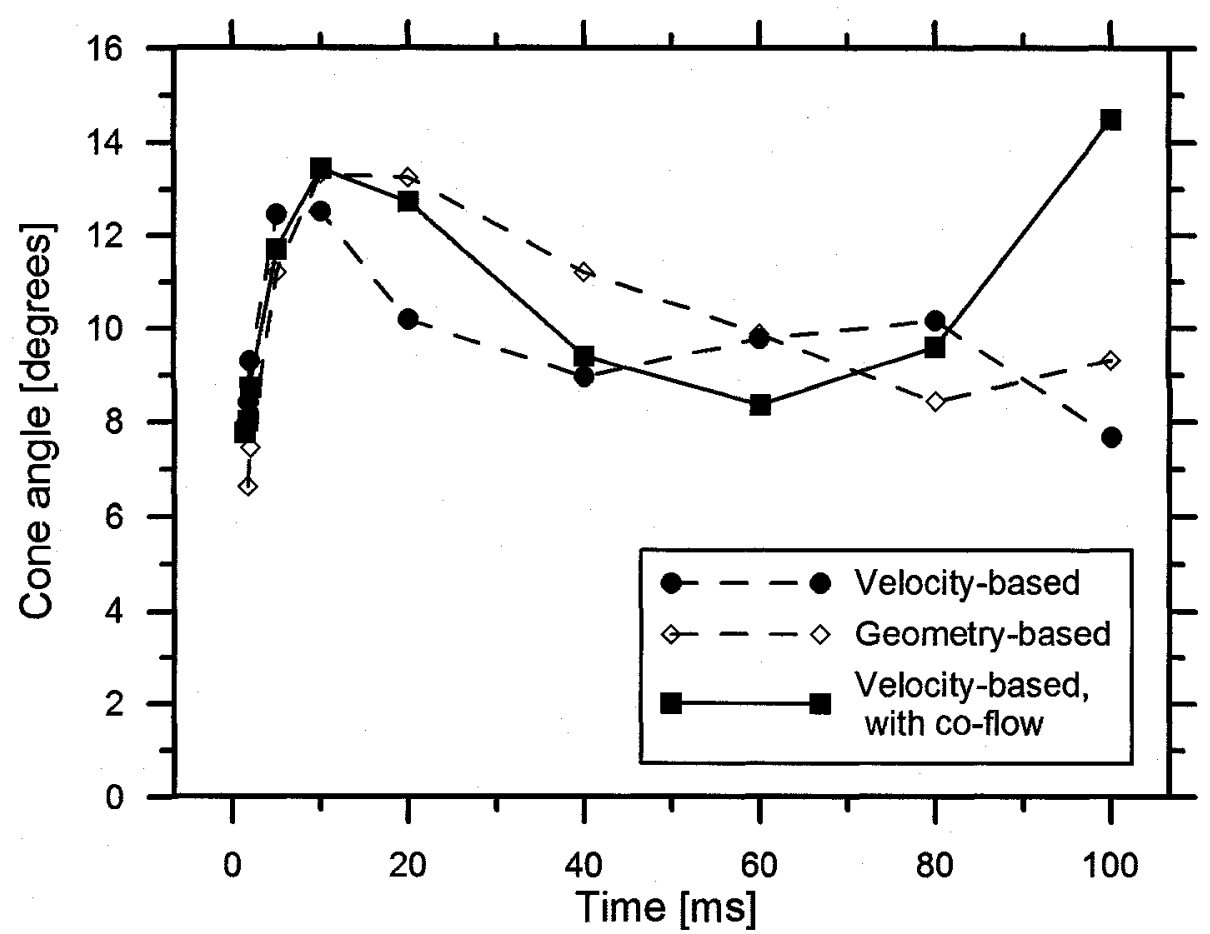

Figure 5.4 - Comparison of the spray cone angle calculated using both calculation methods, as well as the effect of the 30 SLPM co-flow.

Table 5.1 - The minimum, maximum, and time-averaged spray cone angle and direction.

\begin{tabular}{ccccc}
\hline & & \multicolumn{3}{c}{ Cone angle (degrees) } \\
\hline Method & & Minimum & Average & Maximum \\
\hline \hline Geometry & Without co-flow & 6.6 & 10.6 & 13.3 \\
Velocity & Without co-flow & 7.7 & 9.8 & 12.5 \\
Velocity & With co-flow & 7.8 & 10.6 & 14.5 \\
\hline & & \multicolumn{2}{c}{ Spray } & Direction (degrees) \\
\hline Method & & Minimum & Average & Maximum \\
\hline Geometry & Without co-flow & -2.3 & -0.78 & 0.1 \\
Velocity & Without co-flow & -5.5 & -1.2 & 0.2 \\
Velocity & With co-flow & -5.8 & -2.7 & -0.6 \\
\hline
\end{tabular}




\subsection{Spray Issued by an Almost-Full versus an Almost-Empty Canister}

A comparison was also made between the spray emitted by an almost-full canister and that emitted by an almost-empty canister. To determine whether or not significant differences in velocity were present, Student's t-test for independent samples was applied as described in Section 3.7. For each time delay studied, the streamwise spray velocity for the first or second group of 20 actuations was compared with the streamwise spray velocity for the last or next-to-last group of 20 actuations. (The first and last 20 could not always be used since only five canisters were used to take measurements at ten time delays.) Because not all cases used the same time delays (See Table 3.7), spray velocities from ratiopharm-brand pMDIs were thus compared at the nine time delays common to measurements from both brands at four locations in space, for a total of 36 comparisons.

A table of the mean velocities at each point in time and space, as well as the percent difference between the sets (full versus empty), their combined t-score as well as whether or not the difference between them is statistically significant $(p<0.05)$ is given in Table 5.2. Though the average velocities sometimes differ by 25 percent or more, the relatively large standard deviation and small sample size taken into consideration by the t-test show that the velocity of the spray issued from an almost-full canister are statistically indistinguishable from the velocity of the spray issued by an almost-empty canister. 
Table 5.2 - Statistical significance between full and empty canister.

\begin{tabular}{|c|c|c|c|c|c|c|c|}
\hline \multicolumn{8}{|c|}{ At $(x, y)=(2.5 \mathrm{~mm}, 0 \mathrm{~mm})$} \\
\hline $\begin{array}{l}\text { Time } \\
\text { ms }\end{array}$ & $\begin{array}{c}\bar{u} \text { first } 20 \\
\mathrm{~m} / \mathrm{s}\end{array}$ & $\begin{array}{c}\text { s first } 20 \\
m / s\end{array}$ & $\begin{array}{c}\bar{u} \text { last } 20 \\
\mathrm{~m} / \mathrm{s}\end{array}$ & $\begin{array}{c}\mathrm{s} \text { last } 20 \\
\mathrm{~m} / \mathrm{s}\end{array}$ & $\begin{array}{c}\text { Percent } \\
\text { Difference }\end{array}$ & t-score & $\begin{array}{c}\text { Significant? } \\
(p<0.05) \\
(t>2.021)\end{array}$ \\
\hline 1.3 & 58.56 & 14.61 & 61.40 & 7.70 & $4.73 \%$ & 0.77 & NO \\
\hline 2 & 51.37 & 6.39 & 52.37 & 9.54 & $1.93 \%$ & 0.39 & NO \\
\hline 5 & 28.53 & 6.48 & 30.52 & 4.96 & $6.72 \%$ & 1.09 & NO \\
\hline 10 & 18.70 & 6.89 & 21.77 & 7.24 & $15.13 \%$ & 1.37 & NO \\
\hline 20 & 31.23 & 10.72 & 28.26 & 8.32 & $9.98 \%$ & 0.98 & NO \\
\hline 40 & 39.43 & 6.38 & 41.89 & 8.37 & $6.05 \%$ & 1.05 & NO \\
\hline 60 & 44.18 & 7.91 & 46.56 & 8.05 & $5.24 \%$ & 0.94 & NO \\
\hline 80 & 32.18 & 6.21 & 27.58 & 6.62 & $15.41 \%$ & 2.31 & YES \\
\hline 100 & 23.31 & 6.32 & 24.22 & 5.89 & $3.85 \%$ & 0.47 & NO \\
\hline \multicolumn{8}{|c|}{ At $(x, y)=(7.5 \mathrm{~mm}, 0 \mathrm{~mm})$} \\
\hline $\begin{array}{l}\text { Time } \\
\text { ms }\end{array}$ & $\begin{array}{c}\bar{u} \text { first } 20 \\
\mathrm{~m} / \mathrm{s}\end{array}$ & $\begin{array}{c}\mathrm{s} \text { first } 20 \\
\mathrm{~m} / \mathrm{s}\end{array}$ & $\begin{array}{c}\bar{u} \text { last } 20 \\
\mathrm{~m} / \mathrm{s}\end{array}$ & $\begin{array}{c}\text { s last } 20 \\
\mathrm{~m} / \mathrm{s}\end{array}$ & $\begin{array}{c}\text { Percent } \\
\text { Difference }\end{array}$ & t-score & $\begin{array}{c}\text { Significant? } \\
(p<0.05) \\
(t>2.021)\end{array}$ \\
\hline 1.3 & 49.07 & 10.94 & 48.68 & 8.09 & $0.81 \%$ & 0.13 & NO \\
\hline 2 & 44.98 & 6.90 & 44.05 & 7.06 & $2.09 \%$ & 0.42 & NO \\
\hline 5 & 26.68 & 6.49 & 30.82 & 6.30 & $14.40 \%$ & 2.05 & YES \\
\hline 10 & 15.52 & 8.38 & 20.52 & 7.39 & $27.76 \%$ & 2.00 & NO \\
\hline 20 & 27.84 & 6.48 & 24.51 & 7.78 & $12.71 \%$ & 1.47 & NO \\
\hline 40 & 37.96 & 8.57 & 33.84 & 5.63 & $11.46 \%$ & 1.79 & No \\
\hline 60 & 34.10 & 5.70 & 38.78 & 5.81 & $12.85 \%$ & 2.57 & YES \\
\hline 80 & 26.87 & 7.31 & 24.50 & 4.24 & $9.23 \%$ & 1.25 & NO \\
\hline 100 & 20.89 & 5.49 & 19.53 & 4.99 & $6.71 \%$ & 0.82 & No \\
\hline
\end{tabular}




\begin{tabular}{|c|c|c|c|c|c|c|c|}
\hline \multicolumn{8}{|c|}{ At $(x, y)=(12.5 \mathrm{~mm}, 0 \mathrm{~mm})$} \\
\hline $\begin{array}{c}\text { Time } \\
\text { ms }\end{array}$ & $\begin{array}{c}\bar{u} \text { first } 20 \\
\mathrm{~m} / \mathrm{s}\end{array}$ & $\begin{array}{c}\text { s first } 20 \\
\mathrm{~m} / \mathrm{s}\end{array}$ & $\begin{array}{c}\bar{u} \text { last } 20 \\
\mathrm{~m} / \mathrm{s}\end{array}$ & $\begin{array}{c}\text { s last } 20 \\
\mathrm{~m} / \mathrm{s}\end{array}$ & $\begin{array}{c}\text { Percent } \\
\text { Difference }\end{array}$ & t-score & $\begin{array}{c}\text { Significant? } \\
(p<0.05) \\
(t>2.021)\end{array}$ \\
\hline 1.3 & 36.45 & 17.94 & 30.48 & 11.99 & $17.84 \%$ & 1.24 & NO \\
\hline 2 & 35.76 & 6.27 & 40.63 & 8.32 & $12.74 \%$ & 2.06 & YES \\
\hline 5 & 24.85 & 5.91 & 25.72 & 6.66 & $3.43 \%$ & 0.44 & NO \\
\hline 10 & 13.58 & 6.49 & 17.46 & 5.89 & $25.03 \%$ & 1.98 & No \\
\hline 20 & 23.03 & 7.39 & 23.53 & 5.11 & $2.12 \%$ & 0.25 & NO \\
\hline 40 & 32.89 & 6.71 & 34.26 & 5.19 & $4.07 \%$ & 0.72 & NO \\
\hline 60 & 30.01 & 6.40 & 34.02 & 4.94 & $12.52 \%$ & 2.22 & YES \\
\hline 80 & 22.82 & 4.19 & 21.27 & 5.06 & $7.01 \%$ & 1.08 & No \\
\hline 100 & 17.56 & 3.92 & 18.96 & 4.55 & $7.70 \%$ & 1.05 & NO \\
\hline
\end{tabular}

At $(x, y)=(17.5 \mathrm{~mm}, 0 \mathrm{~mm})$

\begin{tabular}{|c|c|c|c|c|c|c|c|}
\hline $\begin{array}{c}\text { Time } \\
\text { ms }\end{array}$ & $\begin{array}{c}\bar{u} \text { first } 20 \\
\mathrm{~m} / \mathrm{s}\end{array}$ & $\begin{array}{c}\text { s first } 20 \\
\mathrm{~m} / \mathrm{s}\end{array}$ & $\begin{array}{c}\bar{u} \text { last } 20 \\
\mathrm{~m} / \mathrm{s}\end{array}$ & $\begin{array}{c}\text { s last } 20 \\
\mathrm{~m} / \mathrm{s}\end{array}$ & $\begin{array}{l}\text { Percent } \\
\text { Difference }\end{array}$ & t-score & $\begin{array}{c}\text { Significant? } \\
(p<0.05) \\
(t>2.021)\end{array}$ \\
\hline 1.3 & \multicolumn{7}{|c|}{ No Spray } \\
\hline 2 & 32.16 & 12.46 & 38.09 & 11.97 & $16.89 \%$ & 2.78 & YES \\
\hline 5 & 20.69 & 6.76 & 24.19 & 6.71 & $15.63 \%$ & 1.83 & NO \\
\hline 10 & 10.73 & 5.75 & 16.12 & 6.36 & $40.11 \%$ & 2.93 & YES \\
\hline 20 & 18.01 & 5.47 & 20.33 & 6.12 & $12.09 \%$ & 1.45 & NO \\
\hline 40 & 31.36 & 5.19 & 30.15 & 4.91 & $3.94 \%$ & 0.58 & NO \\
\hline 60 & 28.81 & 6.19 & 31.46 & 6.94 & $8.81 \%$ & 1.82 & No \\
\hline 80 & 18.87 & 4.15 & 20.14 & 5.05 & $6.50 \%$ & 0.98 & NO \\
\hline 100 & 14.85 & 4.38 & 16.49 & 3.70 & $10.46 \%$ & 1.32 & NO \\
\hline
\end{tabular}

\subsection{Spray Issued by a Ventolin- versus a ratiopharm-Brand Canister}

A comparison similar to that used for full and empty canisters was also applied to the velocity of the spray issued from a pMDI using two different brands of canister. The 
larger sample sizes (100 samples each, rather than the 20 sample sets used for the full versus empty comparison) resulted in smaller percent differences as well as a smaller standard deviation. Once again the streamwise velocity was extracted from the vector velocity flow field at four points in space, at the nine time delays that were measured for both brands. These results are presented in Table 5.3.

Table 5.3 - Statistical significance comparing both brands of canister.

\begin{tabular}{|c|c|c|c|c|c|c|c|}
\hline \multicolumn{8}{|c|}{ At $(x, y)=(2.5 \mathrm{~mm}, 0 \mathrm{~mm})$} \\
\hline $\begin{array}{c}\text { Time } \\
\text { ms }\end{array}$ & $\begin{array}{c}\bar{u} \text { Ventolin } \\
\mathrm{m} / \mathrm{s}\end{array}$ & $\begin{array}{c}\text { s Ventolin } \\
\mathrm{m} / \mathrm{s}\end{array}$ & $\begin{array}{l}\bar{u} \text { ratio } \\
\mathrm{m} / \mathrm{s}\end{array}$ & $\begin{array}{l}\mathrm{s} \text { ratio } \\
\mathrm{m} / \mathrm{s}\end{array}$ & $\begin{array}{c}\text { Percent } \\
\text { Difference }\end{array}$ & t-score & $\begin{array}{c}\text { Significant? } \\
(p<0.05) \\
(t>1.96)\end{array}$ \\
\hline 1.3 & 58.70 & 10.04 & 59.89 & 12.19 & $0.50 \%$ & 0.72 & NO \\
\hline 2 & 58.31 & 10.19 & 58.16 & 9.26 & $0.07 \%$ & 0.11 & NO \\
\hline 5 & 36.78 & 6.47 & 35.42 & 7.69 & $0.94 \%$ & 1.27 & NO \\
\hline 10 & 30.56 & 6.82 & 30.29 & 6.68 & $0.22 \%$ & 0.25 & NO \\
\hline 20 & 36.81 & 8.86 & 36.82 & 7.10 & $0.00 \%$ & 0.00 & NO \\
\hline 40 & 44.75 & 7.39 & 43.42 & 8.84 & $0.75 \%$ & 1.11 & NO \\
\hline 60 & 42.33 & 9.69 & 44.34 & 7.03 & $1.16 \%$ & 1.61 & NO \\
\hline 80 & 30.76 & 5.48 & 33.17 & 6.00 & $1.89 \%$ & 2.94 & YES \\
\hline 100 & 24.76 & 5.51 & 25.23 & 5.88 & $0.47 \%$ & 0.56 & NO \\
\hline
\end{tabular}




\begin{tabular}{cccccccc}
\hline \multicolumn{7}{c}{ At $(\boldsymbol{x}, \boldsymbol{y})=\mathbf{( 7 . 5} \mathbf{~ m m}, \mathbf{0} \mathbf{~ m m})$} \\
\hline Time & $\bar{u}$ Ventolin & $\mathrm{s}$ Ventolin & $\bar{u}$ ratio & $\mathrm{s}$ ratio & Percent & $\mathrm{t}$-score & $\begin{array}{c}\text { Significant? } \\
\mathrm{ms}\end{array}$ \\
$\mathrm{m} / \mathrm{s}$ & $\mathrm{m} / \mathrm{s}$ & $\mathrm{m} / \mathrm{s}$ & $\mathrm{m} / \mathrm{s}$ & Difference & & $\begin{array}{c}(\mathrm{p}>5) \\
(\mathrm{t}>1.96)\end{array}$ \\
\hline 1.3 & 50.52 & 8.45 & 52.79 & 11.19 & $1.10 \%$ & 1.52 & NO \\
2 & 49.79 & 9.03 & 50.69 & 7.96 & $0.45 \%$ & 0.74 & NO \\
5 & 34.43 & 6.75 & 31.80 & 5.92 & $1.99 \%$ & 2.86 & YES \\
10 & 27.53 & 8.45 & 26.39 & 11.19 & $1.06 \%$ & 1.11 & NO \\
20 & 33.55 & 7.69 & 32.00 & 6.69 & $1.18 \%$ & 1.45 & NO \\
40 & 39.23 & 6.35 & 40.13 & 6.70 & $0.57 \%$ & 0.93 & NO \\
60 & 37.54 & 5.95 & 37.53 & 5.97 & $0.00 \%$ & 0.00 & NO \\
80 & 26.11 & 5.04 & 27.94 & 4.79 & $1.69 \%$ & 2.59 & YES \\
100 & 22.45 & 5.81 & 20.84 & 5.34 & $1.85 \%$ & 1.96 & YES
\end{tabular}

At $(x, y)=(12.5 \mathrm{~mm}, 0 \mathrm{~mm})$

\begin{tabular}{|c|c|c|c|c|c|c|c|}
\hline $\begin{array}{c}\text { Time } \\
\text { ms }\end{array}$ & $\begin{array}{c}\bar{u} \text { Ventolin } \\
\mathrm{m} / \mathrm{s}\end{array}$ & $\begin{array}{c}\text { s Ventolin } \\
\mathrm{m} / \mathrm{s}\end{array}$ & $\begin{array}{l}\bar{u} \text { ratio } \\
\mathrm{m} / \mathrm{s}\end{array}$ & $\begin{array}{l}\text { s ratio } \\
\mathrm{m} / \mathrm{s}\end{array}$ & $\begin{array}{c}\text { Percent } \\
\text { Difference }\end{array}$ & t-score & $\begin{array}{c}\text { Significant? } \\
(p<0.05) \\
(t>1.96)\end{array}$ \\
\hline 1.3 & 32.70 & 13.30 & 28.82 & 9.27 & $3.15 \%$ & 2.00 & YES \\
\hline 2 & 43.15 & 9.45 & 44.06 & 8.44 & $0.52 \%$ & 0.71 & NO \\
\hline 5 & 31.88 & 5.97 & 28.97 & 6.84 & $2.39 \%$ & 3.11 & YES \\
\hline 10 & 25.48 & 13.30 & 22.76 & 9.27 & $2.82 \%$ & 2.88 & YES \\
\hline 20 & 29.87 & 6.29 & 29.11 & 6.10 & $0.65 \%$ & 0.83 & NO \\
\hline 40 & 35.39 & 5.68 & 35.54 & 5.87 & $0.11 \%$ & 0.18 & NO \\
\hline 60 & 33.15 & 7.36 & 32.33 & 5.44 & $0.62 \%$ & 0.87 & NO \\
\hline 80 & 22.11 & 4.62 & 23.63 & 5.60 & $1.67 \%$ & 2.06 & YES \\
\hline 100 & 19.21 & 9.27 & 17.29 & 4.43 & $2.63 \%$ & 1.82 & NO \\
\hline
\end{tabular}




\begin{tabular}{|c|c|c|c|c|c|c|c|}
\hline \multicolumn{8}{|c|}{ At $(x, y)=(17.5 \mathrm{~mm}, 0 \mathrm{~mm})$} \\
\hline $\begin{array}{c}\text { Time } \\
\text { ms }\end{array}$ & $\begin{array}{c}\bar{u} \text { Ventolin } \\
\mathrm{m} / \mathrm{s}\end{array}$ & $\begin{array}{c}\text { s Ventolin } \\
\mathrm{m} / \mathrm{s}\end{array}$ & $\begin{array}{l}\bar{u} \text { ratio } \\
\mathrm{m} / \mathrm{s}\end{array}$ & $\begin{array}{l}\text { s ratio } \\
\mathrm{m} / \mathrm{s}\end{array}$ & $\begin{array}{c}\text { Percent } \\
\text { Difference }\end{array}$ & t-score & $\begin{array}{c}\text { Significant? } \\
(p<0.05) \\
(t>1.96)\end{array}$ \\
\hline 1.3 & & & & No Spr & & & \\
\hline 2 & 39.35 & 7.37 & 39.21 & 9.83 & $0.09 \%$ & 0.11 & NO \\
\hline 5 & 29.08 & 6.20 & 27.86 & 6.41 & $1.08 \%$ & 1.35 & NO \\
\hline 10 & 23.11 & 6.66 & 20.81 & 5.83 & $2.62 \%$ & 2.53 & YES \\
\hline 20 & 27.94 & 6.59 & 26.61 & 5.36 & $1.21 \%$ & 1.51 & NO \\
\hline 40 & 33.23 & 6.13 & 31.87 & 5.78 & $1.04 \%$ & 1.56 & NO \\
\hline 60 & 30.82 & 5.00 & 29.00 & 4.97 & $1.52 \%$ & 2.48 & YES \\
\hline 80 & 20.73 & 4.45 & 21.10 & 4.18 & $0.44 \%$ & 0.60 & NO \\
\hline 100 & 16.35 & 3.67 & 15.80 & 3.54 & $0.85 \%$ & 1.04 & NO \\
\hline
\end{tabular}

\subsection{Average Turbulent Kinetic Energy}

The normalized average turbulent kinetic energy field is defined as:

$$
\frac{k_{t k e}}{\bar{V}^{2}}=\frac{\overline{u^{\prime 2}}+\overline{v^{\prime 2}}+\overline{w^{\prime 2}}}{2 \cdot \bar{V}^{2}}
$$

If it is assumed that the flow is similar in the $y$ and $z$ directions then it is possible to calculate simplify equation 5.1 by assuming that $w^{, 2}=v^{, 2}$ :

$$
\frac{k_{t k e}}{\bar{V}^{2}}=\frac{\overline{u^{\prime 2}}+2 \overline{v^{\prime 2}}}{2 \cdot \bar{V}^{2}}
$$

This allows the average turbulent kinetic energy to be calculated using PIV measurement data. However, the result of this equation is very sensitive to spurious vectors, so extra post-processing is required to ensure no spurious vectors remain. Toward this end, a post-processing step was added wherein any vectors with a peak ratio (see Section 3.8.3) of less than 1.5 was removed. Any vectors surrounded by fewer than 
seven valid vectors were also removed. Also, during the calculation of the turbulence kinetic energy results were only retained at locations where more than $75 \%$ of the measurements contained valid vectors. An example of the average turbulent kinetic energy field for the pMDI spray issuing directly into ambient air is presented in Figure 5.5. Appendix E contains additional plots of the calculated turbulent kinetic energy fields for the remaining time delays.

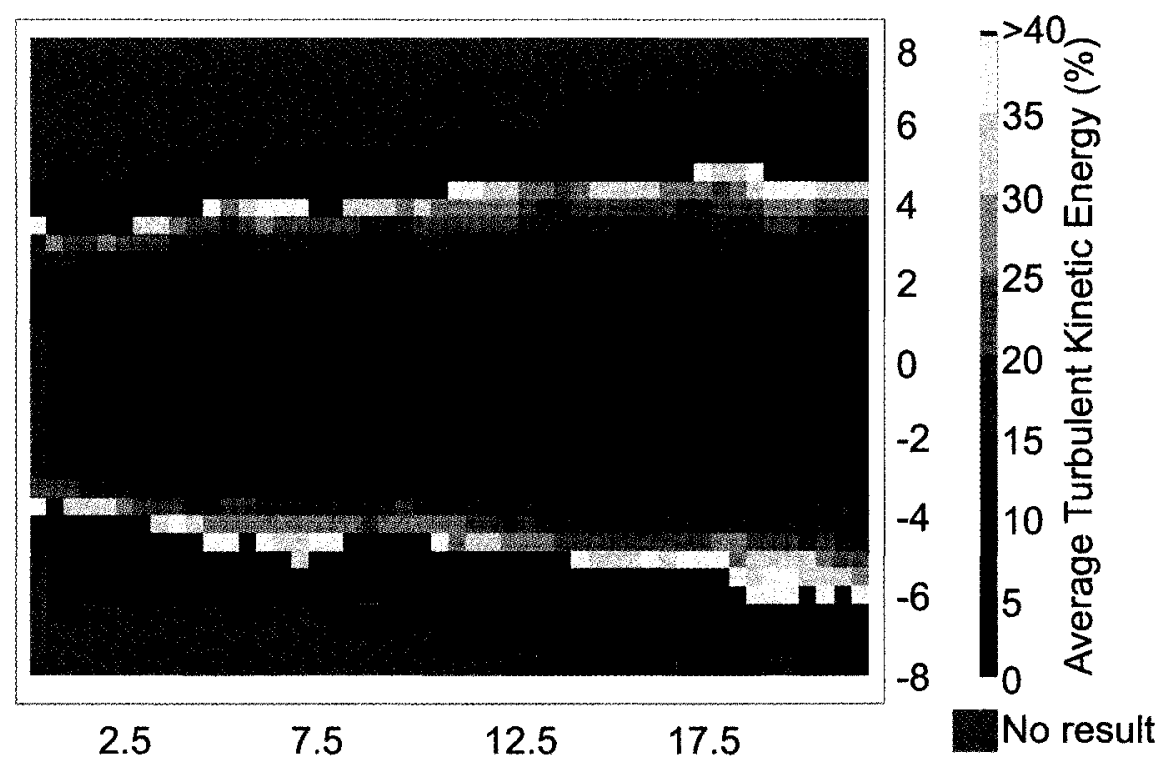

Figure 5.5 - Average turbulence kinetic energy calculated using equation 5.2. ratiopharm, $60 \mathrm{~ms}$

\subsection{Curve-fit of Profiles for Future Use}

Even after extensive data reduction, it is impossible to foresee all possible future uses for the data presented herein. It is believed that the most immediate application of these data is to serve as inlet conditions for numerical studies of pMDI flows. Toward this end, vertical profiles of streamwise and vertical velocities at the most upstream location for which all data is available were fitted parametrically. (The averaged velocity data used 
for the fits is included in Appendix F.) This will allow future researchers to insert the given equations into a CFD code, assigning a realistic initial velocity to particles entering the computational grid. Researchers who choose to simulate the spray in its entirety (beginning at the nozzle) could also use these curves to validate their results at the mouthpiece outlet.

The most upstream streamwise location for which no gaps exist in the data is the plane at $x=1 \mathrm{~mm}$, parallel to the mouthpiece plane. This plane is $26 \mathrm{~mm}$ downstream of the pMDI nozzle. A commercial curve-fitting software package, TableCurve2D (SYSTAT Software Inc, Chicago, Illinois, USA) was used to select a suitable equation type to fit the profiles as well as to determine the parameters providing the best fit for each time delay. For the vertical profiles of streamwise velocity, a $\chi^{2}$ distribution was found to fit the data well:

$$
u=a+\frac{b\left(\frac{y-c+d(e-2)}{d}\right)^{\left(\frac{e}{2}-1\right)} \exp \left(-\frac{y-c+d(e-2)}{2 d}\right)}{(e-2)^{\left(\frac{e}{2}-1\right)} \exp \left(-\frac{e}{2}+1\right)}
$$

This representation of the $\chi^{2}$ distribution includes a y-intercept, so the streamwise velocity, $u$ is a function of five parameters, $(a, b, c, d$ and $e)$ as well as the vertical location, measured from the centreline. Because the $\chi^{2}$ distribution is non-symmetric, values of $y$ greater than 0 are above the centerline, while values of $y$ less than 0 are below the centerline. The curve fit parameters for the streamwise velocity, $u$, are presented in Table 5.4. 
Table 5.4 - Curve fit parameters for streamwise velocity, $u$, as a function of time.

\begin{tabular}{cccccc}
\hline $\mathbf{t}(\mathbf{m s})$ & $\mathbf{a}$ & $\mathbf{b}$ & $\mathbf{c}$ & $\mathbf{d}$ & $\mathbf{e}$ \\
\hline 1.3 & -0.4618 & 63.1562 & 0.0294 & 0.0329 & 1219.541 \\
1.7 & -0.4839 & 60.4735 & -0.1537 & 0.0578 & 488.162 \\
2 & -0.5697 & 60.1256 & -0.4231 & 0.0618 & 427.8082 \\
5 & -0.5339 & 36.5326 & -1.5576 & 0.0397 & 1955.589 \\
10 & -1.1987 & 31.8937 & -2.4806 & 0.0395 & 2543.23 \\
20 & -0.1855 & 37.5558 & -2.0256 & 0.0353 & 2560.173 \\
40 & 0.4487 & 44.1196 & -0.8781 & 0.0272 & 2560.827 \\
60 & 0.7495 & 43.9614 & -0.2862 & 0.0418 & 756.2221 \\
80 & 0.2363 & 34.3316 & 0.1357 & 0.0322 & 1321.061 \\
100 & 0.089 & 20.6099 & -0.1261 & 0.0257 & 2530.561 \\
\hline
\end{tabular}

The vertical profiles of vertical velocity were less easily characterized. Generally, the vertical velocity decreases with increasing distance from the centerline, with opposite sign on opposite sides of the centerline. A rational polynomial was used to represent these profiles:

$$
v=\frac{\left(a+c y+e y^{2}+g y^{3}+i y^{4}\right)}{\left(1+b y+d y^{2}+f y^{3}+h y^{4}\right)}
$$

Unfortunately, to accurately capture the unusual shape of these profiles, nine parameters were required. The curve fit parameters for the vertical velocity are presented in Table 5.5. 
Table 5.5 - Curve fit parameters for vertical velocity, $v$ as a function of time.

\begin{tabular}{cccccccccc}
\hline $\mathbf{t}$ (ms) & $\mathbf{a}$ & $\mathbf{b}$ & $\mathbf{c}$ & $\mathbf{d}$ & $\mathbf{e}$ & $\mathbf{f}$ & $\mathbf{g}$ & $\mathbf{h}$ & $\mathbf{i}$ \\
\hline 1.3 & -0.3624 & -0.5133 & 2.748 & 0.131 & 0.7199 & 0.3369 & -0.189 & 0.07283 & -0.0367 \\
1.7 & 0.7229 & 0.1151 & 2.379 & 0.2002 & 0.3193 & 0.151 & -0.07298 & 0.03399 & -0.00904 \\
2 & -0.2942 & -0.1604 & 2.057 & -0.01602 & 0.2937 & 0.1455 & -0.03266 & 0.05525 & 0 \\
5 & -1.077 & -0.06154 & 1.472 & 0.08495 & -0.05095 & 0.02889 & -0.03911 & 0.003825 & 0 \\
10 & -0.9095 & -0.1342 & 1.025 & -0.04489 & -0.2048 & -0.00273 & 0.001858 & 0.004667 & 0 \\
20 & -0.9321 & 0.03713 & 1.806 & 0.1338 & -0.0903 & 0.0476 & -0.03494 & 0.007595 & 0 \\
40 & -0.5074 & 0.2355 & 1.245 & 0.1148 & 0.06432 & 0.02471 & -0.03536 & 0.00591 & 0 \\
60 & -0.5395 & 0.3982 & 1.312 & 0.1682 & 0.5282 & 0.01379 & -0.02946 & 0.000791 & -0.00873 \\
80 & 0.05602 & 0.04222 & 0.4572 & -0.103 & 0.1497 & -0.00017 & -0.03247 & 0.006767 & 0 \\
100 & 0.4201 & 0.1762 & 0.6598 & -0.00909 & 0.2059 & 0.006157 & -0.02156 & 0.004154 & -0.00311 \\
\hline
\end{tabular}

These curves were generated using ratiopharm-brand salbutamol sulphate canisters in a Ventolin-brand casing with a nozzle diameter of $0.5 \mathrm{~mm}$ actuated into quiescent air. The data used to generate the curves, as well as the curve fits themselves, are presented in Figures 5.6 and 5.7. Note that some data is repeated from one plot to the next to allow easy comparison. Though statistically significant differences exist between the velocities measured with and without a co-flow or spacer, the measured results are similar enough that these curve-fits could easily be adapted to represent spray in a co-flow if desired. 

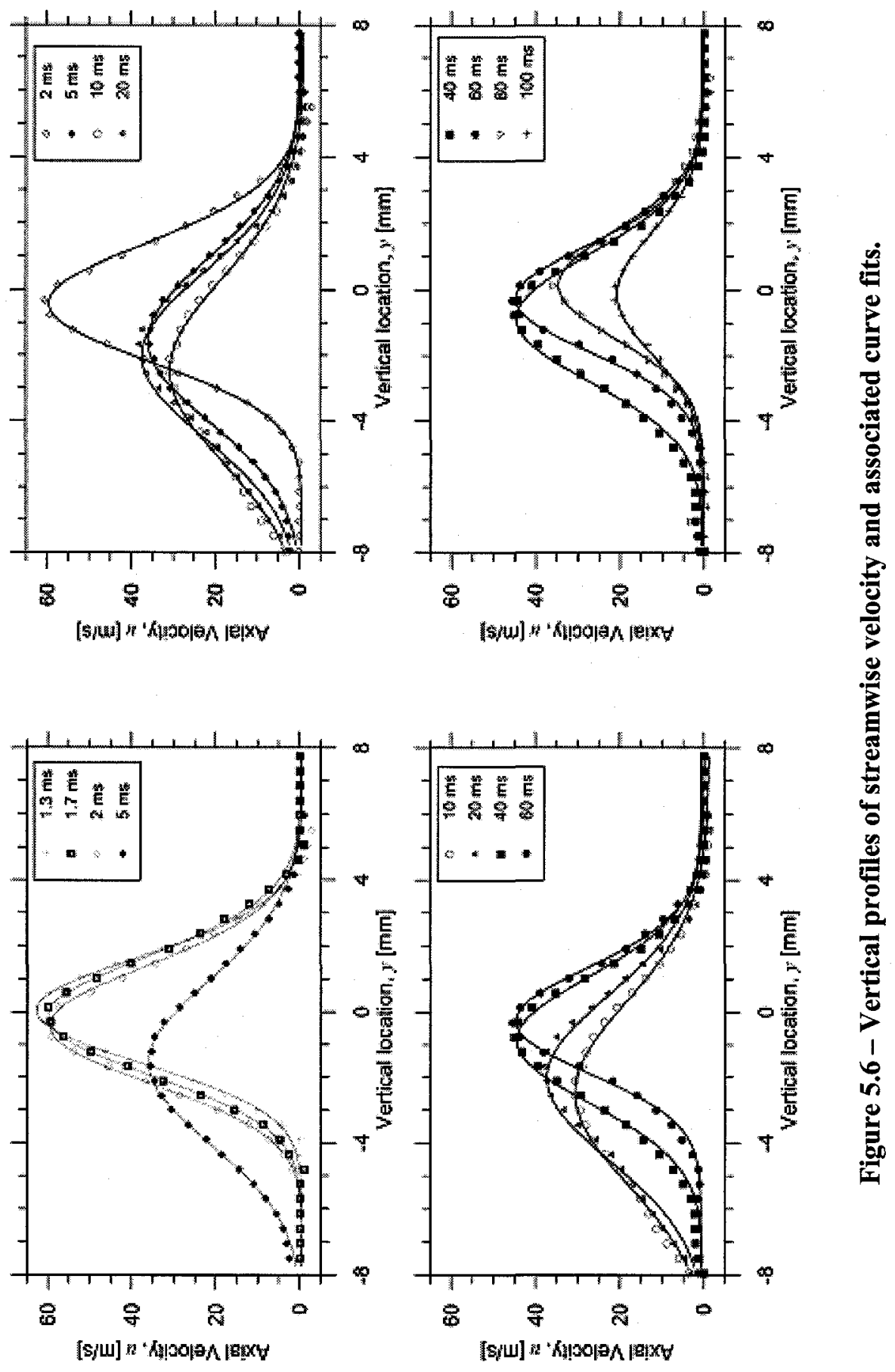

 


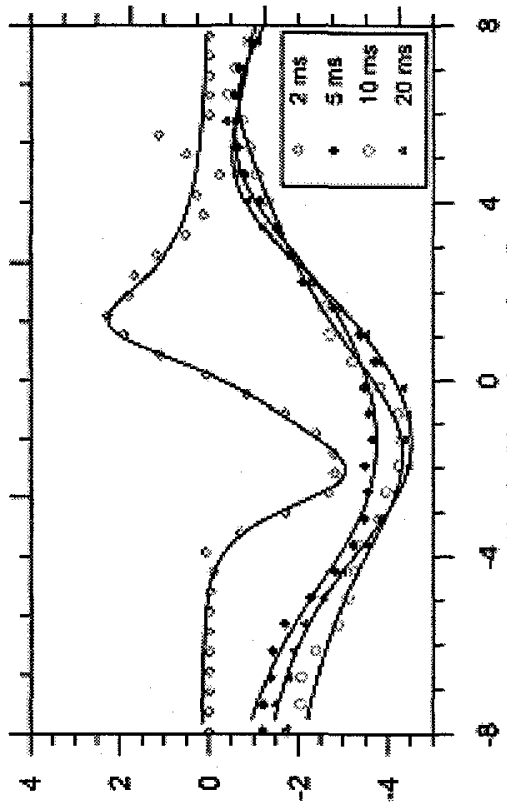

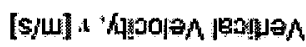

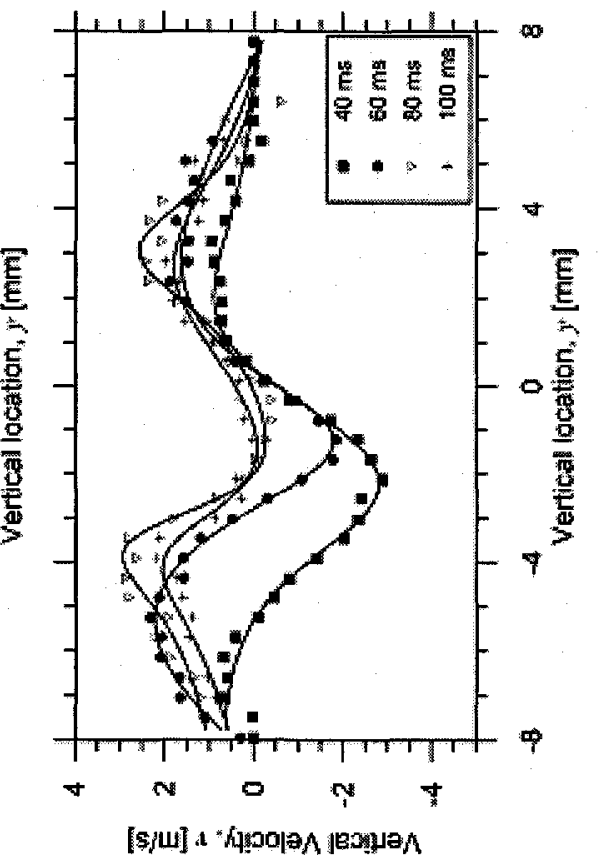



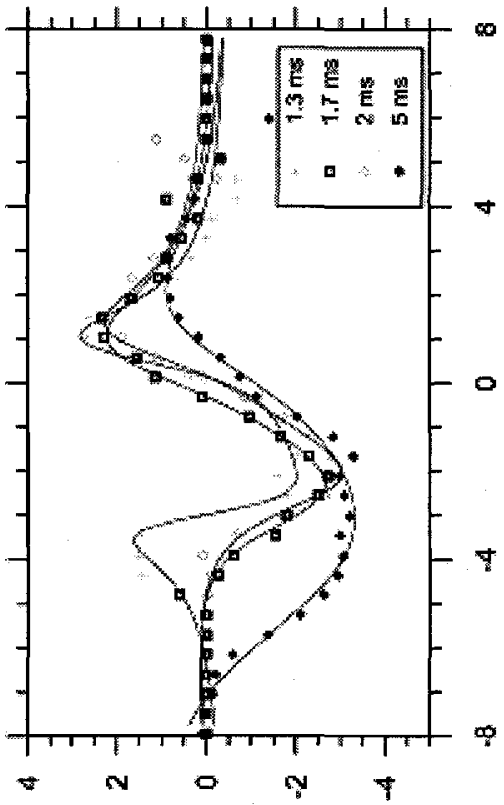

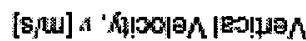

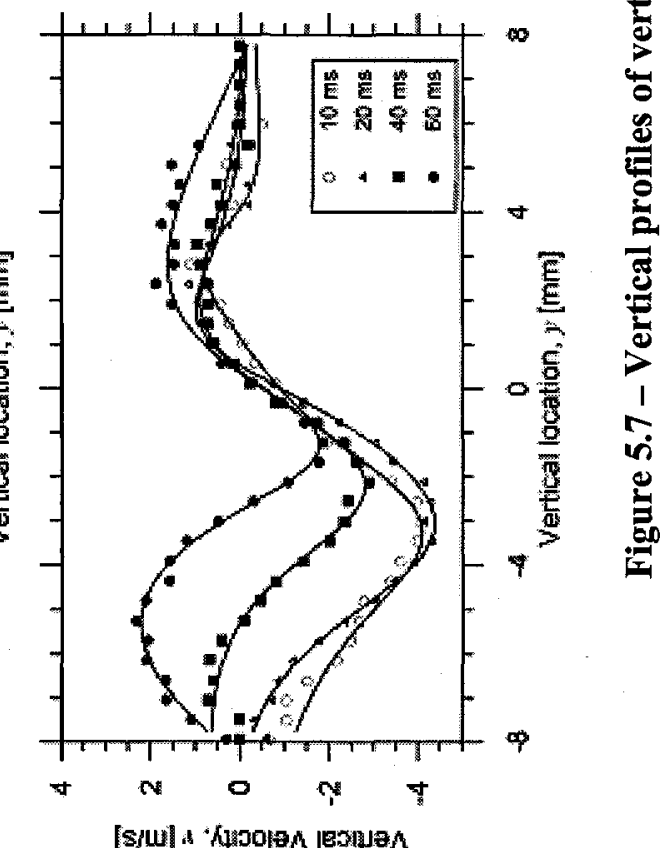




\section{Chapter 6: Conclusions and Recommendations}

\subsection{Conclusions}

The results presented in this thesis characterize the spray particle velocity in a $20 \mathrm{~mm} \times 16 \mathrm{~mm}$ window at the exit of the pMDI mouthpiece with a spatial resolution of $0.4 \mathrm{~mm}$. Spray particle velocity was measured at ten discrete times after pMDI actuation, from $1.3 \mathrm{~ms}$ to $100 \mathrm{~ms}$. Comparisons were made between canister brands as well as between a full and an empty canister. The effect of a secondary air flow of 30 SLPM, both with and without an attached valved chamber was examined.

To the author's knowledge, the research presented in this thesis represents the most detailed spatial characterization of velocity of the pMDI spray available in the open literature. The results support four major contributions to the past, present and future of the study of pMDI flow. First, it has been further reinforced that the spray velocities are bimodal in time, as demonstrated by Wigley (2002) as well as experimentally (but not numerically) by Dunbar (1996, 1997a, 1997b). Second, the research presented here agrees with velocity maps recently published by Kakade (2007) in the placement of the peak velocity below the horizontal $(x z)$ plane. Third, it has been shown that no statistically significant differences exist between i) two brands of canister, and ii) a full versus an empty canister. Finally, a major contribution of this research is the facilitation 
of future numerical simulations through the presentation of velocity profiles as parameterized curve fits, as well as the reduction of cases requiring study via statistical analysis. These curves represent the velocity of spray particles along the vertical (y) axis, $1 \mathrm{~mm}$ downstream of the mouthpiece outlet plane of a HFA-propelled salbutamol sulphate pMDI with a nozzle diameter of $0.5 \mathrm{~mm}$.

\subsection{Recommendations for Future Work}

There is one major avenue of research that arises from the results presented in this thesis, as well as many other minor directions which might be taken. The major recommendation for future work is the numerical simulation of inhalatory flow with the release and tracking of particles given an initial velocity representing a pMDI spray. This would be a major undertaking, involving complex numerical simulations.

The minor recommendations fall under two main categories: new work based on this research, and new work expanding this research. New work based on this research could include a simulation of the pMDI spray alone, either upstream or downstream of the currently measured field of view. This would allow comparison with existing work as well as facilitate future numerical simulations in these regions. New work expanding upon this research could include experimental measurement of the pMDI in measurement planes other than the vertical (sagittal) plane. 


\section{Appendix A: Uncertainty in Instantaneous Velocity Measurement using PIV}

Using upper case to denote the image plane, and lower case the object plane, the basic equation used in PIV calculations is:

$$
v=\frac{\Delta X}{M \Delta t}
$$

Where $M$ is the image magnification, $\Delta X$ is the displacement, $\Delta t$ is the interframe time, and $v$ is the velocity. The total error involved in calculating the velocity, $v$ is thus:

$$
\delta v=\sqrt{\left(\frac{\partial v}{\partial M}\right)^{2}(\delta M)^{2}+\left(\frac{\partial v}{\partial(\Delta X)}\right)^{2}(\delta(\Delta X))^{2}+\left(\frac{\partial v}{\partial(\Delta t)}\right)^{2}(\delta(\Delta t))^{2}}
$$

The partial derivatives can be calculated directly from equation A.1, as follows:

$$
\begin{aligned}
& \frac{\partial v}{\partial M}=-\frac{\Delta X}{M^{2} \Delta t} \\
& \frac{\partial v}{\partial(\Delta X)}=\frac{1}{M \Delta t} \\
& \frac{\partial v}{\partial(\Delta t)}=-\frac{\Delta X}{M \Delta t^{2}}
\end{aligned}
$$

The uncertainty in the magnification, displacement, and interframe time terms can be estimated based on the fundamental uncertainties associated with each term. 


\section{A.1 Magnification Uncertainty, $e_{1}$}

The uncertainty in the magnification terms comes as a result of improper alignment of the calibration plate with the laser sheet (Ramasamy and Leishman, 2007). Assuming the laser sheet and calibration plate have been aligned as closely as possible at the centre of the sheet, there will be errors associated with a rotation of the calibration plate about the $x$-axis $(\alpha)$, the $y$-axis $(\beta)$ as well as a translation in the $z$-axis $(z)$. The total uncertainty associated with the calculation of the magnification is thus:

$$
\delta M=\sqrt{\left(\frac{\partial M}{\partial \alpha}\right)^{2}(\delta \alpha)^{2}+\left(\frac{\partial M}{\partial \beta}\right)^{2}(\delta \beta)^{2}+\left(\frac{\partial M}{\partial z}\right)^{2}(\delta z)^{2}}
$$

Once the calibration has been performed, the camera will be imaging the true laser-sheet plane. Thus the width and height of the calibration plate projected onto the laser-sheet plane are expressed as:

$$
x_{C P}=\frac{x_{C P-P}}{\cos \alpha} \quad y_{C P}=\frac{y_{C P-P}}{\cos \alpha}
$$

Where $x_{\mathrm{CP}}$ and $y_{\mathrm{CP}}$ are the true width and height of the calibration plate, in metres, and $x_{\text {CP-P }}$ and $y_{\text {CP-P }}$ are the projected width and height of the calibration plate, also in metres. In the image plane, this becomes:

$$
X_{C P}=\frac{X_{C P-P}}{\cos \alpha} \quad Y_{C P}=\frac{Y_{C P-P}}{\cos \alpha}
$$

The magnification is defined as the ratio of the imaged size of the calibration plate to the true size of the calibration plate:

$$
M=\frac{X_{C P}}{x_{C P}}
$$


But with a non-zero rotation of the plate, the magnification of experimental measurements taken in the laser sheet plane will be:

$$
M=\frac{X_{C P}}{x_{C P} \cos \alpha}
$$

The rate of change of magnification with respect to $\alpha$ can now be expressed as follows:

$$
\frac{\partial M}{\partial \alpha}=\frac{-X_{C P} \sin \alpha}{x_{C P} \cos ^{2} \alpha}=-M \sec \alpha \tan \alpha
$$

Similarly, for a misalignment of rotation about the $y$-axis, the rate of change of magnification with respect to $\beta$ can be expressed as:

$$
\frac{\partial M}{\partial \beta}=-M \sec \beta \tan \beta
$$

To calculate the rate of change of magnification with respect to $z$, the magnification must first be expressed as a function of $z$. Using the notation demonstrated in Figure A.1, the magnification can also be defined as the ratio of the distances of the image and object planes from the focal point of the lens:

$$
M=\frac{d_{i}}{d_{o}}=\frac{d_{i}}{z-d_{i}}=\frac{\left(\frac{X_{C P}}{2 \tan \gamma}\right)}{z-\left(\frac{X_{C P}}{2 \tan \gamma}\right)}=\frac{X_{C P}}{2 z \tan \gamma-X_{C P}}
$$




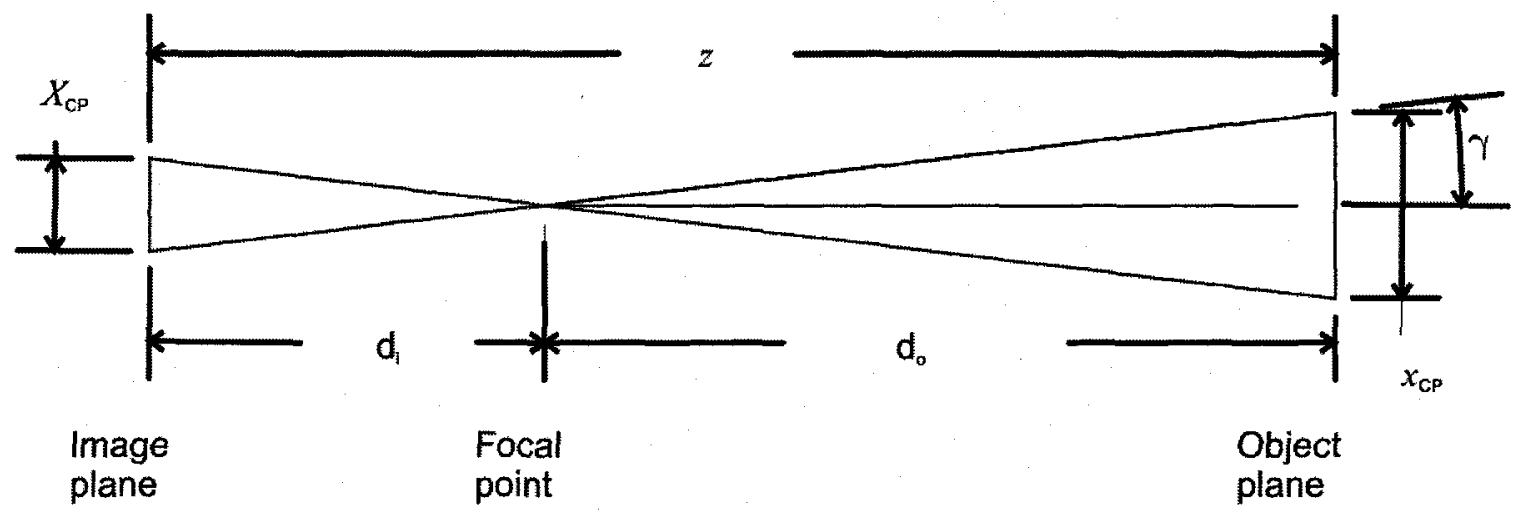

Figure A.1 - Diagram of image and object planes.

Its derivative with respect to $z$ is thus:

$$
\left.\frac{\partial M}{\partial z}\right|_{x}=\frac{-2 X_{C P} \tan \gamma_{x}}{\left(2 z \tan \gamma-X_{C P}\right)^{2}}
$$

But when the camera faces the image plane directly (i.e. the line from the centre of the image through the focus to the centre of the object is perpendicular to both the image and object planes) the angle $\gamma_{\mathrm{x}}$ can be defined as:

$$
\gamma=\tan ^{-1}\left(\frac{X_{C P}}{2 d_{i}}\right)
$$

Which allows equation A.12 to be simplified to:

$$
\left.\frac{\partial M}{\partial z}\right|_{x}=-\frac{d_{i}}{\left(z-d_{i}\right)^{2}}=\left.\frac{\partial M}{\partial z}\right|_{y}
$$

Thus the rate of change of the magnification with respect to $z$ does not depend on the direction of measurement. Since any measured displacement will involve a displacement in both the $y$-and $x$-axes, this derivative must be doubled: 


$$
\frac{\partial M}{\partial z}=\frac{2 d_{i}}{\left(z-d_{i}\right)^{2}}
$$

The total uncertainty in the measurement of the magnification as expressed in equation A.4 can now be expressed as:

$$
\delta M=\sqrt{(-M \sec \alpha \tan \alpha)^{2}(\delta \alpha)^{2}+(-M \sec \beta \tan \beta)^{2}(\delta \beta)^{2}+\left(\frac{2 d_{i}}{\left(z-d_{i}\right)^{2}}\right)^{2}(\delta z)^{2}}
$$

Because it is impossible to know $\alpha$ and $\beta$ other than by assuming a maximum possible value, $\delta \alpha$ and $\delta \beta$ are assumed to equal 1 while $\alpha$ and $\beta$ are assumed to have maximum values of 1 degree. The uncertainty in the $z$-axis, $\delta z$, is assumed to equal half of the laser sheet thickness, or $\approx 1 \mathrm{~mm}$. The focal length of the lens, $d_{i}$, is $105 \mathrm{~mm}$ and the distance between the sensor and the image is about $315 \mathrm{~mm}$. The magnification was calculated as 0.50. This gives a total uncertainty in the calculation of the magnification of:

$$
\mathrm{e}_{1}=0.0132
$$

\section{A.2 Image Correction Uncertainty, $\mathbf{e}_{2}$}

The uncertainty in the calculation of the displacement of a particle must account for uncertainty due to the use of a third-degree polynomial to map locations in the object plane to corresponding locations in the image plane. This approximate mapping performed by the FlowMaster software reported the standard deviation of the error in fitting the 20 calibration dots. This can be used to calculate the precision error in determining the displacement of a particle: 


$$
P_{X}=\frac{t_{95} s_{X}}{\sqrt{N}}
$$

Where $s_{X}$ is the standard deviation, $N$ is the number of measurements made (i.e. the number of dots) and $t_{95}$ is a student t-factor for $95 \%$ certainty and $N-1$ degrees of freedom. For Ventolin-brand pMDIs actuated into open air, this yields a precision error of:

$$
P_{X}=0.106 \text { pixels }
$$

When measuring a displacement, this error will be encurred in determining both the initial and final location of the particle:

$$
\mathrm{e}_{2}=2 P_{X}=0.212 \text { pixels }=1.57 \mu \mathrm{m}
$$

\section{A.3 Non-Zero Laser Pulse Length Uncertainty, $\mathbf{e}_{3}$}

Another uncertainty in determining displacement depends on the laser pulse timing, and the assumption that the laser pulse illuminates the particles for a sufficiently short time so as to freeze particle motion. Since the laser pulse lasts at most $5 \mathrm{~ns}$, the image of a particle moving $100 \mathrm{~m} / \mathrm{s}$ will travel $0.25 \mu \mathrm{m}$, or $3.3 \%$ of the length of a pixel, during the pulse event. Like the image correction uncertainty, this applies to both the initial and final position of a particle, and thus must be multiplied by two.

$$
\mathrm{e}_{3}=0.50 \mu \mathrm{m}
$$

\section{A.4 Laser Timing Uncertainty, $\mathrm{e}_{4}$}

The only uncertainty directly affecting the time term in Equation (A.2) involves the precision of the interframe time, $\Delta t$. As stated in its accompanying owner's manual 
(LaVision 2006c), the laser timing card has a temporal resolution of $50 \mathrm{~ns}$. It is thus assumed to have a precision error of half of its resolution whilst triggering both the first and second laser pulses. Thus the maximum possible uncertainty is:

$$
\mathrm{e}_{4}=50 \mathrm{~ns}
$$

\section{A.5 Noise and Loss of Particles Uncertainty, $e_{5}$ and $e_{6}$}

The random error associated with PIV measurements was found by Boillot and Prasad (1996) and Prasad et al. (1992) to scale with the particle image diameter, $\mathrm{d}_{\tau}$ :

$$
\mathrm{e}_{5 / 6}=c d_{\tau}
$$

Where the constant $c$ is between 0.05 and 0.07 . Because the scattered, rather than the reflected light is being imaged by the camera, the minimum particle image diameter $\left(d_{\tau}\right)$ will be a function of the true particle diameter, the magnification, and the diffractionlimited minimum image diameter:

$$
d_{\tau}=\sqrt{\left(M d_{p}\right)^{2}+d_{d i f f}^{2}}
$$

The diffraction limited minimum image diameter, as provided by Raffel (1998, p.40):

$$
d_{\text {diff }}=2.44 f(M+1) \lambda
$$

Where $\lambda$ is the wavelength of light, $(532 \mathrm{~nm})$ and $f$ is the focal number of the lens, defined as the ratio between the focal length and the aperture diameter. A focal number of 2.8 was used for all PIV images. To make a worst-case estimate of this error, a relatively large particle size of $70 \mu \mathrm{m}$ was used for $d_{p}$. This corresponds to an imaged particle size of 5 pixels, $66 \%$ larger than most pixels appear in the images themselves. This returns a random error of: 


$$
\mathrm{e}_{5 / 6}=(0.07)(35.5 \mu \mathrm{m})=2.48 \mu \mathrm{m}
$$

\section{A.6 Pixel-Locking Uncertainty, $e_{7}$}

A common concern in PIV measurements is the tendency of the correlation algorithm to calculate displacements that are integer pixel values, called peak- or pixel-locking. Prasad et al. (1992) suggest that to minimize the total error resulting from random error and peak-locking error, $d_{\tau}$ should be equal to $\approx 2$ pixels, at which point the pixel-locking error is negligible compared to the random error. As previously mentioned, a true particle diameter of $70 \mu \mathrm{m}$ will create an imaged particle diameter of $\approx 5$ pixels. However, judging from the images of individual particles at the periphery of the spray, as well as the results of Dunbar (1996) and Graham (1995) it is more likely that the true particle diameter of most particles will fall between 1 and $10 \mu \mathrm{m}$, yielding imaged particle diameters between 0.75 and 1.0 pixels. The lack of pixel-locking can be confirmed by plotting a histogram of the fractional pixel displacement of the vectors in a calculated flow field, as shown for a sample instantaneous measurement in Figure A.2.

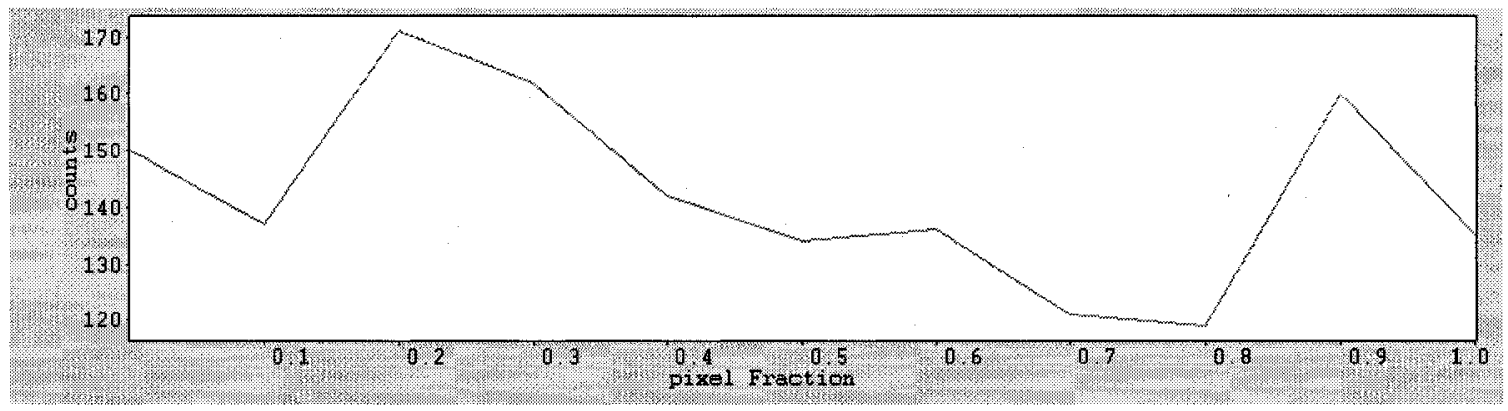

Figure A.2 - A histogram of (vector displacement mod 1), in pixels.

If pixel-locking was present in a measurement, there would be a tendency for the histogram to have greater counts at the left and right end of the graph. This trend was not 
seen upon inspection of histograms of various measurements at all time delays. Thus this error term can be neglected:

$$
\mathrm{e}_{7} \approx 0
$$

\section{A.7 Velocity Gradient Bias Uncertainty, $\mathbf{e}_{8}$}

Another source of error in PIV involves velocity gradient bias. This arises when strong velocity gradients within an IW cause the correlation peak to widen, introducing uncertainty. For evenly spaced IWs, Ramasamy and Leishman (2007) suggest the interframe time and IW size should be chosen such that:

$$
\Delta V_{i, i+1} \Delta t \leq d_{\tau}
$$

Where $\Delta \mathrm{V}_{\mathrm{i}, \mathrm{i}+1}$ is the difference in velocity between neighbouring IWs. Assuming a true particle size of $5 \mu \mathrm{m}$, the recommended maximum velocity difference between adjacent nodes is $5.0 \mathrm{~m} / \mathrm{s}$. Looking at the average velocity fields of Ventolin-brand pMDIs actuated into open air there are a number of vectors that pass this limit, as outlined in Table A.1. Though it may seem like the measurements at the earliest time delays will suffer from a velocity gradient bias, it is important to note that the mean and median of the large vectors are only at most $33 \%$ above the recommended limit, and that this is exactly the kind of error that the translation and deformation of IWs discussed earlier is designed to correct. Overall it is believed that velocity gradient bias error does not have an important effect on the measurement uncertainty.

$$
\mathrm{e}_{8} \approx 0
$$


Table A.1 - The number of vectors possibly affected by velocity gradient bias.

\begin{tabular}{ccccccc}
\hline Time & \# large & \# valid & \% vectors & \multicolumn{2}{c}{ Properties of set of large vectors } \\
\cline { 6 - 7 } delay & vectors & vectors & above limit & Mean & Median & Std deviation \\
\hline 1.3 & 301 & 1388 & $22 \%$ & 6.85 & 6.66 & 1.24 \\
2 & 257 & 2788 & $9.2 \%$ & 6.26 & 6.14 & 0.92 \\
5 & 2 & 3502 & $0.1 \%$ & 5.09 & 5.09 & 0.00 \\
10 & 0 & 3440 & --- & --- & -- & -- \\
20 & 7 & 3310 & $0.2 \%$ & 5.32 & 5.31 & 0.04 \\
40 & 69 & 3202 & $2.2 \%$ & 5.41 & 5.29 & 0.34 \\
60 & 81 & 3348 & $2.4 \%$ & 5.93 & 5.75 & 0.64 \\
80 & 2 & 3262 & $0.1 \%$ & 5.13 & 5.13 & 0.00 \\
100 & 0 & 3260 & --- & -- & -1 & - \\
\hline
\end{tabular}

\section{A.8 Traceability of Seed Particles}

In measurements where a co-flow of air was used, the co-flow was seeded with olive oil droplets to allow measurement of the flow in areas where there were no spray particles present. Being much denser than the surrounding air, these tracer particles have a less than perfect ability to track the flow.

Based on Melling (1997), as well as Hjelmfelt and Mockros (1966), the ability of particles to track a flow consisting of homogeneous, stationary turbulence with a maximum turbulence frequency of interest $\omega_{c}$ can be characterised by the relative amplitude, $\eta$, and the phase response, $\beta$, of the particle and fluid motions. For a 
turbulence frequency and particle diameter of interest, it must then be decided if the relative amplitude and phase response are sufficient for accurate flow tracking.

To determine the maximum turbulence frequency of interest, a length scale is required. The spatial resolution of the PIV calculations (with $50 \%$ overlap) is $0.4 \mathrm{~mm}$. Conservatively, a length scale of $0.2 \mathrm{~mm}$ will be used.

$$
\mathrm{L}=0.2 \mathrm{~mm}
$$

Using the Taylor hypothesis, a length scale can be used to approximate a time, and thus a frequency, associated with turbulence of this scale.

$$
f=\frac{u_{\text {avg }}}{\mathrm{L}}=21 \mathrm{kHz}
$$

Where $u_{\text {avg }}$ is taken to be $4.18 \mathrm{~m} / \mathrm{s}$, the largest value measured in the co-flow-only measurement data presented in Section 4.3. This indicates that in order for the olive oil seed particles to track the flow, they must be able to accurately follow velocity fluctuations at a frequency of $21 \mathrm{kHz}$. Returning now to the work of Melling (1997) as well as Hjelmfelt and Mockros (1966), the relative amplitude and phase response of the seed particles in the air flow can be determined as follows:

$$
\begin{gathered}
\eta=\left\{\left(1+f_{2}\right)^{2}+f_{2}^{2}\right\}^{1 / 2} \\
\beta=\tan ^{-1}\left\{\frac{f_{2}}{1+f_{1}}\right\}
\end{gathered}
$$

Where the functions $f_{1}$ and $f_{2}$ are represented by: 


$$
\begin{gathered}
f_{1}=\frac{\left[1+\frac{9}{\sqrt{2}(s+1 / 2)} N_{s}\right]\left[\frac{1-s}{s+1 / 2}\right]}{\frac{81}{(s+1 / 2)^{2}}\left[2 N_{s}^{2}+\frac{N_{s}}{\sqrt{2}}\right]^{2}+\left[1+\frac{9}{\sqrt{2}(s+1 / 2)} N_{s}\right]^{2}} \\
f_{2}=\frac{\left[\frac{9(1-s)}{(s+1 / 2)^{2}}\right]\left[2 N_{s}^{2}+\frac{N_{s}}{\sqrt{2}}\right]}{\frac{81}{(s+1 / 2)^{2}}\left[2 N_{s}^{2}+\frac{N_{s}}{\sqrt{2}}\right]^{2}+\left[1+\frac{9}{\sqrt{2}(s+1 / 2)} N_{s}\right]^{2}}
\end{gathered}
$$

Where $s$ is the density ratio between the particles and the fluid:

$$
s=\rho_{p} / \rho_{f}
$$

And the Stokes number is given by:

$$
N_{s}=\left(\frac{v_{f}}{\omega d_{p}^{2}}\right)^{1 / 2}
$$

Olive oil has a density of approximately $970 \mathrm{~kg} / \mathrm{m}^{3}$ (Melling, 1997). Assuming the particles have a MMAD of of $1.0 \mu \mathrm{m}$ (Fischer et al., 1994; Willert et al., 1996) and using the frequency of $21 \mathrm{kHz}$ determined in Equation A.28 to specify a cutoff frequency $\omega$, it can be determined that the relative amplitude and phase shift between the particle velocity and the fluid velocity will be:

$$
\begin{gathered}
\eta=1.0 \\
\beta=-2.4^{\circ}
\end{gathered}
$$

thus indicating that the olive oil particles are suitable for flow tracking. 


\section{A.9 Total Measurement Uncertainty}

The total measurement uncertainty can now be calculated using equation A.2 and the calculated values for each elemental error.

$$
\begin{gathered}
\delta v=\sqrt{\left(\frac{\partial v}{\partial M}\right)^{2}\left(\mathrm{e}_{1}\right)^{2}+\left(\frac{\partial v}{\partial(\Delta X)}\right)^{2}\left(\mathrm{e}_{2}^{2}+\mathrm{e}_{3}^{2}+\mathrm{e}_{5 / 6}^{2}\right)+\left(\frac{\partial v}{\partial(\Delta t)}\right)^{2}\left(\mathrm{e}_{4}\right)^{2}} \\
\delta v=\sqrt{\left(-\frac{\Delta X}{M^{2} \Delta t}\right)^{2}\left(1.74 \cdot 10^{-4}\right)+\left(\frac{1}{M \Delta t}\right)^{2}\left(8.63 \cdot 10^{-12} \mathrm{~m}^{2}\right)+\left(-\frac{\Delta X}{M \Delta t^{2}}\right)^{2}\left(6.2 \cdot 10^{-16} \mathrm{~s}^{2}\right)}(\mathrm{A} .36)
\end{gathered}
$$

The nominal magnification was measured to be 0.500 and the nominal interframe time is $1.2 \mu \mathrm{s}$. This further simplifies equation A.36 to:

$$
\delta v=\sqrt{24.6\left(\frac{m}{s}\right)^{2}+0.00244 v^{2}}
$$

Table A.3 gives the velocity, as well as the absolute and percent uncertainty, for various values of displacement. 
Table A.3 - Measurement uncertainty for selected values of displacement

\begin{tabular}{cccc}
\hline$\Delta \mathbf{X}$, pixels & $\mathbf{v}(\mathrm{m} / \mathrm{s})$ & $\delta \mathbf{v}(\mathrm{m} / \mathrm{s})$ & $\delta \mathrm{v} / \mathbf{v}$ \\
\hline 0.5 & 6.16 & 4.97 & 0.81 \\
1 & 12.3 & 4.99 & 0.41 \\
2 & 24.6 & 5.10 & 0.21 \\
3 & 37.0 & 5.28 & 0.14 \\
4 & 49.3 & 5.52 & 0.11 \\
5 & 61.6 & 5.82 & 0.09 \\
6 & 73.9 & 6.16 & 0.08 \\
7 & 86.3 & 6.53 & 0.08 \\
8 & 98.6 & 6.95 & 0.07 \\
\hline
\end{tabular}




\section{Appendix B: Uncertainty in Time Delay}

Due to the difficulty associated with determining the amount of time that passes between the actuation of the pMDI and the triggering of the Nd:YAG laser, it is more prudent to simply sum the maximum possible amount of time that each step in the process might take.

\section{B.1 Time For He:Ne Laser Beam to Travel from Canister to Photodiode}

The simplest way to calculate the time that passes between the canister being blocked and the beam no longer illuminating the photodiode is to divide the distance traveled by the beam by the speed of light. The distance between the edge of the canister closest to the photodiode and the photodiode is, at most, $1 \mathrm{~cm}$. Taking the speed of light in air to be approximately the same as that in a vacuum, $c=3.0 \times 10^{8} \mathrm{~m} / \mathrm{s}$, this error term becomes:

$$
\mathrm{e}_{1}=0.03 \mathrm{~ns}
$$

\section{B.2 Rise Time of the Photodiode}

The rise time of the photodiode depends on the resistance of the external load. Following the instructions in the DaVis Hardware manual (LaVision 2006c), a $50 \Omega$ BNC terminator was connected in parallel with the triggering circuit. Assuming the terminator resistance has a $10 \%$ uncertainty, the maximum expected rise time for the photodiode is:

$$
\mathrm{e}_{2}=2.25 \mathrm{~ns}
$$




\section{B.3 Rise Time of the Voltage Inverter}

To provide a rising-edge trigger for the PIV computer, a SN7406 solid-state voltage inverter was connected in series with the photodiode. The maximum switching time of the inverter is:

$$
\mathrm{e}_{3}=15 \mathrm{~ns}
$$

\section{B.4 Time for Signal to Travel from pMDI to PIV Computer}

A $10 \mathrm{~m}$ long coaxial cable was used to transmit the triggering signal from the pMDI to the PIV computer. Assuming that the signal is transmitted at a speed near the speed of light, the time required for it to travel $10 \mathrm{~m}$ is:

$$
\mathrm{e}_{4}=33 \mathrm{~ns}
$$

\section{B.5 Resolution of the PIV Computer Timing Card}

The timing card inside the PIV computer has a resolution of $50 \mathrm{~ns}$. Assuming that the signal arrives immediately after a clock cycle, the maximum precision error is:

$$
\mathrm{e}_{5}=50 \mathrm{~ns}
$$




\section{B.6 Operation of Metering Valve}

The significant variation measured from one actuation to the next is a result of the imperfect nature of the pMDI actuation mechanism. Slight variations in its operation have significant effects on the resulting spray velocity flow field. Though this represents a significant uncertainty in the measurements, it is inherent in the device design, and thus is impossible to eliminate and difficult to estimate. Thus the error term associated with the operation of the metering valve is neglected:

$$
\mathrm{e}_{6} \approx 0
$$

\section{B.6 Maximum Possible Delay}

Thus the maximum possible delay that could occur between the time the pMDI canister is actuated and the time at which the PIV computer receives the triggering signal and begins the data acquisition process is:

$$
\mathrm{e}_{\mathrm{total}}=101 \mathrm{~ns}
$$




\section{Appendix C: RMS Velocity at Selected Times and Locations}

The measured spray velocities varied greatly from one actuation to the next. Tables

C.1-C.9 show the root-mean squared difference of individual measurements to the mean, defined as:

$$
\mathrm{RMS}=\sqrt{\frac{1}{n-1} \sum_{i=1}^{n}\left(u_{i}-\bar{u}\right)^{2}}
$$

Where $n$ is the number of measurements with valid vectors at a given location, $u_{i}$ is the instantaneous streamwise or vertical velocity and $\bar{u}$ is the mean streamwise or vertical velocity.

Tables C. 1 - C.9 give RMS at various locations within the spray at nine time delays measured using a Ventolin-brand canister. 
Table C.1 - RMS of streamwise and vertical velocity, in m/s. Ventolin, $1.3 \mathrm{~ms}$.

\begin{tabular}{c|ccccccccc}
\hline & \multicolumn{2}{|c}{$\mathbf{2 . 5} \mathbf{~ m m}$} & \multicolumn{2}{c}{$\mathbf{7 . 5} \mathbf{~ m m}$} & \multicolumn{2}{c}{$12.5 \mathbf{m m}$} & \multicolumn{2}{c}{$17.5 \mathbf{~ m m}$} \\
\cline { 2 - 8 } & Stream. & Vert. & Stream. & Vert. & Stream. & Vert. & Stream. & Vert. \\
\hline $5 \mathrm{~mm}$ & 6.9 & 4.9 & 7.9 & 6.3 & N/A & N/A & N/A & N/A \\
$2.5 \mathrm{~mm}$ & 11.7 & 6.0 & 11.6 & 5.9 & 10.6 & 4.4 & N/A & N/A \\
$0 \mathrm{~mm}$ & 10.0 & 3.6 & 8.5 & 3.9 & 13.3 & 2.8 & 12.0 & 4.6 \\
$-2.5 \mathrm{~mm}$ & 10.0 & 5.6 & 12.5 & 5.9 & 10.7 & 4.6 & N/A & N/A \\
$-5 \mathrm{~mm}$ & N/A & N/A & N/A & N/A & N/A & N/A & N/A & N/A \\
\hline
\end{tabular}

Table C.2 - RMS of streamwise and vertical velocity, in $\mathrm{m} / \mathrm{s}$. Ventolin, $2 \mathrm{~ms}$.

\begin{tabular}{c|ccccccccc}
\hline & \multicolumn{2}{c}{$\mathbf{x}$} & $\mathbf{2 . 5} \mathbf{~ m m}$ & \multicolumn{2}{c}{$\mathbf{7 . 5} \mathbf{~ m m}$} & \multicolumn{2}{c}{$12.5 \mathbf{~ m m}$} & \multicolumn{2}{c}{$17.5 \mathbf{~ m m}$} \\
\cline { 2 - 9 } & Stream. & Vert. & Stream. & Vert. & Stream. & Vert. & Stream. & Vert. \\
\hline $5 \mathrm{~mm}$ & 4.2 & 3.8 & 5.8 & 3.7 & 6.3 & 4.6 & 8.7 & 5.9 \\
$2.5 \mathrm{~mm}$ & 8.8 & 4.4 & 10.3 & 5.4 & 7.6 & 5.4 & 7.3 & 4.8 \\
$0 \mathrm{~mm}$ & 9.8 & 3.5 & 8.7 & 4.4 & 8.8 & 4.9 & 7.6 & 5.0 \\
$-2.5 \mathrm{~mm}$ & 10.2 & 4.4 & 9.2 & 3.9 & 9.1 & 4.7 & 8.8 & 4.3 \\
$-5 \mathrm{~mm}$ & 6.1 & 4.0 & 7.6 & 4.9 & 8.2 & 4.7 & 6.6 & 5.4 \\
\hline
\end{tabular}


Table C.3 - RMS of streamwise and vertical velocity, in $\mathrm{m} / \mathrm{s}$. Ventolin, $5 \mathrm{~ms}$.

\begin{tabular}{cccccccccc}
\hline & \multicolumn{2}{c}{$\boldsymbol{2} \mathbf{2 . 5} \mathbf{~ m m}$} & \multicolumn{2}{c}{$\mathbf{7 . 5} \mathbf{~ m m}$} & \multicolumn{2}{c}{$12.5 \mathrm{~mm}$} & \multicolumn{2}{c}{$17.5 \mathrm{~mm}$} \\
\cline { 2 - 8 } & Stream. & Vert. & Stream. & Vert. & Stream. & Vert. & Stream. & Vert. \\
\hline $5 \mathrm{~mm}$ & 4.5 & 2.9 & 4.0 & 3.3 & 3.8 & 2.8 & 4.6 & 3.6 \\
$2.5 \mathrm{~mm}$ & 6.3 & 3.3 & 5.2 & 3.8 & 5.7 & 3.0 & 5.3 & 3.3 \\
$0 \mathrm{~mm}$ & 7.3 & 3.4 & 7.2 & 3.6 & 6.9 & 3.3 & 6.1 & 3.7 \\
$-2.5 \mathrm{~mm}$ & 5.6 & 3.6 & 6.1 & 3.5 & 6.4 & 4.4 & 6.5 & 3.3 \\
$-5 \mathrm{~mm}$ & 6.6 & 3.9 & 6.4 & 4.1 & 7.5 & 4.1 & 7.0 & 3.7 \\
\hline
\end{tabular}

Table C.4 - RMS of streamwise and vertical velocity, in $\mathrm{m} / \mathrm{s}$. Ventolin, $10 \mathrm{~ms}$.

\begin{tabular}{c|ccccccccc}
\hline & \multicolumn{2}{c}{$\boldsymbol{x}$} & \multicolumn{2}{c}{$\mathbf{2 . 5} \mathbf{~ m m}$} & \multicolumn{2}{c}{$\mathbf{7 . 5} \mathbf{~ m m}$} & \multicolumn{2}{c}{$\mathbf{1 2 . 5} \mathbf{~ m m}$} & \multicolumn{2}{c}{$\mathbf{1 7 . 5} \mathbf{~ m m}$} \\
\cline { 2 - 9 } & Stream. & Vert. & Stream. & Vert. & Stream. & Vert. & Stream. & Vert. \\
\hline $5 \mathrm{~mm}$ & 4.4 & 3.1 & 3.9 & 2.4 & 3.8 & 2.7 & 4.0 & 2.8 \\
$2.5 \mathrm{~mm}$ & 8.8 & 3.4 & 6.7 & 3.5 & 6.6 & 3.2 & 5.9 & 2.8 \\
$0 \mathrm{~mm}$ & 8.8 & 3.0 & 7.7 & 3.0 & 6.5 & 2.9 & 6.4 & 22.9 \\
$-2.5 \mathrm{~mm}$ & 13.1 & 3.7 & 7.1 & 3.7 & 7.1 & 3.6 & 6.3 & 3.8 \\
$-5 \mathrm{~mm}$ & 6.1 & 4.2 & 5.6 & 4.2 & 5.6 & 4.0 & 5.9 & 3.2 \\
\hline
\end{tabular}


Table C.5 - RMS of streamwise and vertical velocity, in $\mathrm{m} / \mathrm{s}$. Ventolin, $20 \mathrm{~ms}$.

\begin{tabular}{|c|c|c|c|c|c|c|c|c|}
\hline$x$ & \multicolumn{2}{|c|}{$2.5 \mathrm{~mm}$} & \multicolumn{2}{|c|}{$7.5 \mathrm{~mm}$} & \multicolumn{2}{|c|}{$12.5 \mathrm{~mm}$} & \multicolumn{2}{|c|}{$17.5 \mathrm{~mm}$} \\
\hline & Stream. & Vert. & Stream. & Vert. & Stream. & Vert. & Stream. & Vert. \\
\hline $5 \mathrm{~mm}$ & 3.5 & 2.3 & 3.6 & 2.9 & 3.4 & 2.6 & 4.7 & 2.8 \\
\hline $2.5 \mathrm{~mm}$ & 6.8 & 3.5 & 6.5 & 3.3 & 7.2 & 3.3 & 5.8 & 2.7 \\
\hline $0 \mathrm{~mm}$ & 10.5 & 3.5 & 8.4 & 3.3 & 6.3 & 3.2 & 6.2 & 3.7 \\
\hline$-2.5 \mathrm{~mm}$ & 5.8 & 3.3 & 6.3 & 3.4 & 6.1 & 3.7 & 6.1 & 3.9 \\
\hline$-5 \mathrm{~mm}$ & 6.1 & 3.2 & 5.1 & 4.1 & 5.6 & 3.7 & 5.8 & 3.5 \\
\hline
\end{tabular}

Table C.6 - RMS of streamwise and vertical velocity, in m/s. Ventolin, $40 \mathrm{~ms}$.

\begin{tabular}{|c|c|c|c|c|c|c|c|c|}
\hline$x$ & \multicolumn{2}{|c|}{$2.5 \mathrm{~mm}$} & \multicolumn{2}{|c|}{$7.5 \mathrm{~mm}$} & \multicolumn{2}{|c|}{$12.5 \mathrm{~mm}$} & \multicolumn{2}{|c|}{$17.5 \mathrm{~mm}$} \\
\hline & Stream. & Vert. & Stream. & Vert. & Stream. & Vert. & Stream. & Vert. \\
\hline $5 \mathrm{~mm}$ & 3.3 & 2.6 & 4.4 & 3.1 & 3.9 & 2.6 & 4.7 & 3.2 \\
\hline $2.5 \mathrm{~mm}$ & 8.6 & 3.3 & 6.8 & 3.3 & 6.6 & 3.0 & 6.5 & 3.2 \\
\hline $0 \mathrm{~mm}$ & 8.3 & 3.5 & 7.5 & 3.0 & 6.0 & 2.5 & 6.3 & 3.1 \\
\hline$-2.5 \mathrm{~mm}$ & 7.1 & 2.6 & 7.1 & 3.2 & 6.8 & 3.3 & 6.1 & 3.9 \\
\hline$-5 \mathrm{~mm}$ & 5.0 & 2.8 & 8.3 & 3.4 & 6.0 & 3.0 & 6.3 & 3.1 \\
\hline
\end{tabular}


Table C.7 - RMS of streamwise and vertical velocity, in $\mathrm{m} / \mathrm{s}$. Ventolin, $60 \mathrm{~ms}$.

\begin{tabular}{c|ccccccccc}
\hline & \multicolumn{2}{c}{$\boldsymbol{x}$} & \multicolumn{2}{c}{$\mathbf{2 . 5} \mathbf{~ \mathbf { m }}$} & \multicolumn{2}{c}{$\mathbf{7 . 5} \mathbf{~ \mathbf { m }}$} & \multicolumn{2}{c}{$\mathbf{1 2 . 5} \mathbf{~ m m}$} & \multicolumn{2}{c}{$\mathbf{1 7 . 5} \mathbf{~ m m}$} \\
\cline { 2 - 9 } & Stream. & Vert. & Stream. & Vert. & Stream. & Vert. & Stream. & Vert. \\
\hline $5 \mathrm{~mm}$ & 4.8 & 2.4 & 3.8 & 2.5 & 4.0 & 2.8 & 4.3 & 2.8 \\
$2.5 \mathrm{~mm}$ & 6.4 & 3.4 & 6.8 & 2.8 & 6.4 & 3.0 & 5.3 & 3.1 \\
$0 \mathrm{~mm}$ & 7.2 & 3.0 & 5.9 & 3.2 & 7.4 & 3.2 & 5.1 & 3.0 \\
$-2.5 \mathrm{~mm}$ & 4.6 & 2.5 & 5.7 & 2.7 & 6.0 & 2.7 & 5.8 & 3.3 \\
$-5 \mathrm{~mm}$ & 3.3 & 2.4 & 3.7 & 2.6 & 4.0 & 2.2 & 4.7 & 2.5 \\
\hline
\end{tabular}

Table C.8 - RMS of streamwise and vertical velocity, in $\mathrm{m} / \mathrm{s}$. Ventolin, $80 \mathrm{~ms}$.

\begin{tabular}{c|ccccccccc}
\hline & \multicolumn{2}{c}{$\mathbf{2}$} & $\mathbf{2 . 5} \mathbf{m m}$ & \multicolumn{2}{c}{$\mathbf{7 . 5} \mathbf{~ m m}$} & \multicolumn{2}{c}{$12.5 \mathrm{~mm}$} & \multicolumn{2}{c}{$17.5 \mathrm{~mm}$} \\
\cline { 2 - 8 } & Stream. & Vert. & Stream. & Vert. & Stream. & Vert. & Stream. & Vert. \\
\hline $5 \mathrm{~mm}$ & 4.8 & 3.2 & 4.8 & 3.0 & 4.7 & 2.7 & 5.0 & 3.1 \\
$2.5 \mathrm{~mm}$ & 6.6 & 3.2 & 6.6 & 3.2 & 4.8 & 2.9 & 4.6 & 3.0 \\
$0 \mathrm{~mm}$ & 6.5 & 2.8 & 5.3 & 3.0 & 5.1 & 3.0 & 4.9 & 3.0 \\
$-2.5 \mathrm{~mm}$ & 5.7 & 3.2 & 5.2 & 2.9 & 4.8 & 2.9 & 5.3 & 2.9 \\
$-5 \mathrm{~mm}$ & 4.0 & 3.4 & 2.9 & 3.1 & 3.9 & 2.9 & 3.6 & 2.4 \\
\hline
\end{tabular}


Table C.9 - RMS of streamwise and vertical velocity, in $\mathrm{m} / \mathrm{s}$. Ventolin, $100 \mathrm{~ms}$.

\begin{tabular}{c|ccccccccc}
\hline & $\boldsymbol{x}$ & \multicolumn{2}{|c}{$\mathbf{2 . 5} \mathbf{~ m m}$} & \multicolumn{2}{c}{$\mathbf{7 . 5} \mathbf{~ m m}$} & \multicolumn{2}{c}{$12.5 \mathbf{~ m m}$} & \multicolumn{2}{c}{$17.5 \mathbf{~ m m}$} \\
\cline { 2 - 8 } & Stream. & Vert. & Stream. & Vert. & Stream. & Vert. & Stream. & Vert. \\
\hline $5 \mathrm{~mm}$ & 4.2 & 4.4 & 3.7 & 4.0 & 4.4 & 3.7 & 4.6 & 3.6 \\
$2.5 \mathrm{~mm}$ & 6.4 & 4.0 & 5.8 & 3.3 & 5.8 & 3.2 & 5.2 & 3.2 \\
$0 \mathrm{~mm}$ & 5.7 & 3.4 & 5.4 & 3.4 & 4.7 & 3.7 & 3.9 & 3.0 \\
$-2.5 \mathrm{~mm}$ & 5.2 & 4.2 & 5.5 & 4.2 & 5.1 & 3.5 & 9.9 & 3.1 \\
$-5 \mathrm{~mm}$ & 3.3 & 3.6 & 3.7 & 3.0 & 4.2 & 3.8 & 3.5 & 2.6 \\
\hline
\end{tabular}




\section{Appendix D: Velocity at Spacer Outlet}

Measurements were taken at the outlet of an Aerochamber add-on spacer device. All measurements were made with a 30 SLPM secondary flow of air to ensure the valve at the outlet of the device would be held open during pMDI actuation. The measurement field of view relative to the pMDI is shown in Figure D.1, followed by vertical profiles of streamwise velocity superimposed over colour plots showing the magnitude of velocity for 10 time delays ranging from $2.5-100 \mathrm{~ms}$ after pMDI actuation.

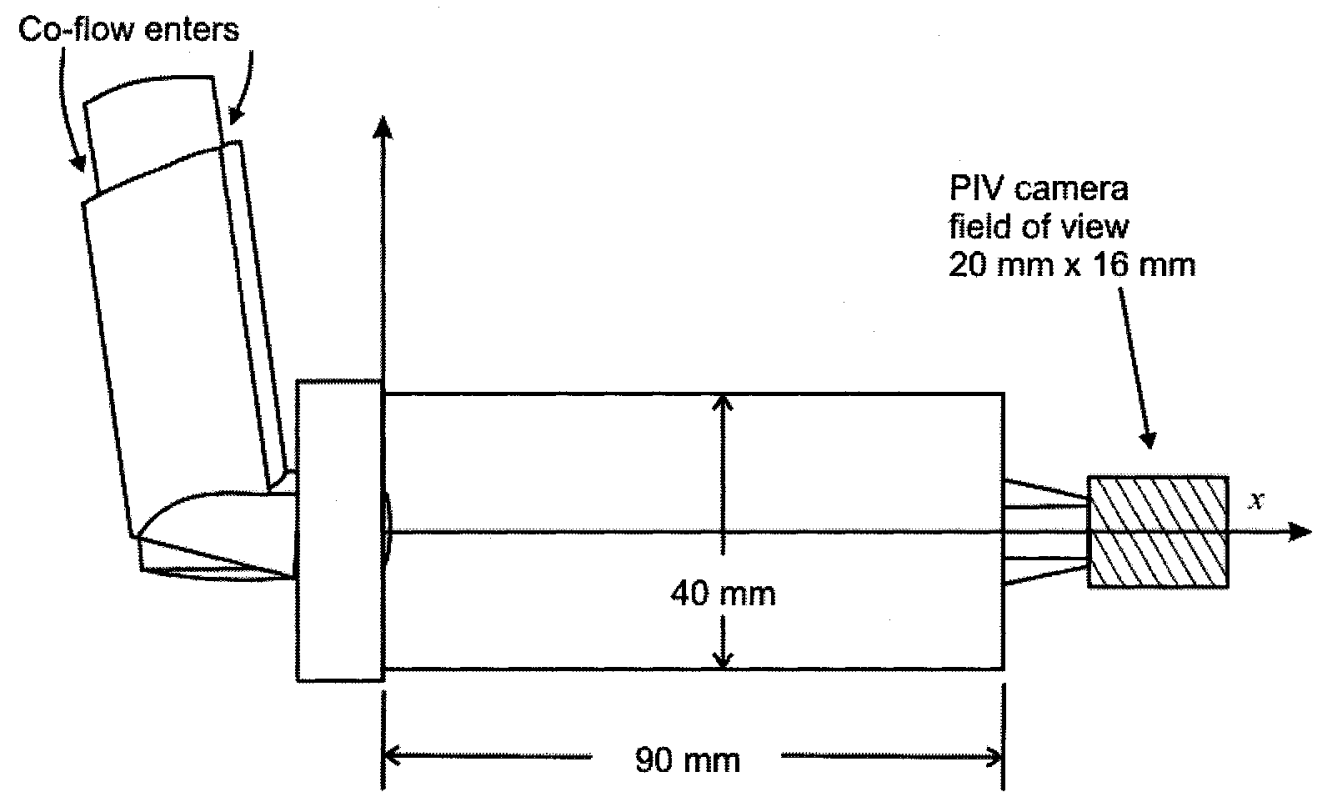

Figure D.1 - The field of view used for PIV measurements at the outlet of the add-on device. 


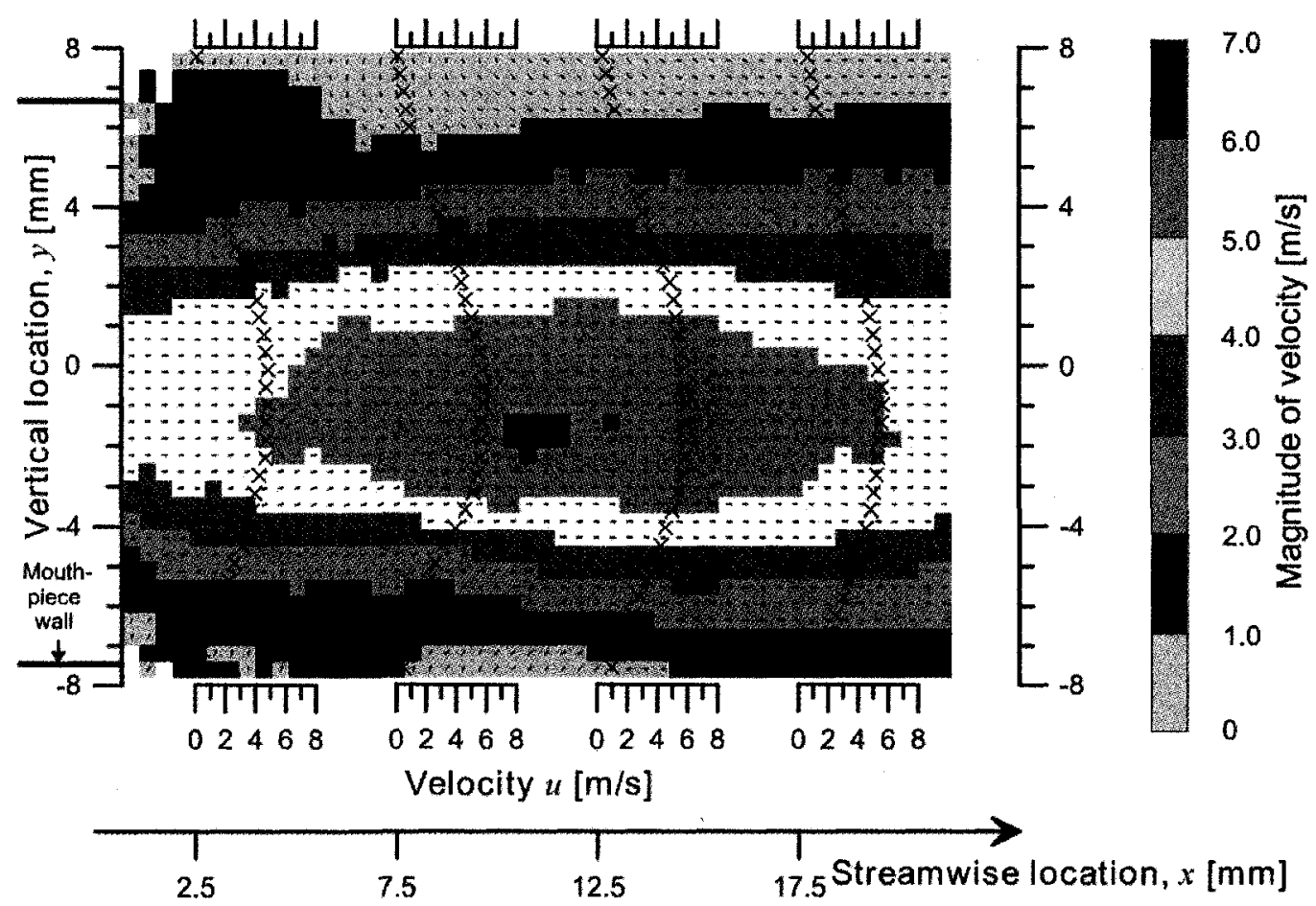

Figure D.2 - Average vector velocity flow field. Ventolin, spacer outlet, $2.5 \mathrm{~ms}$.

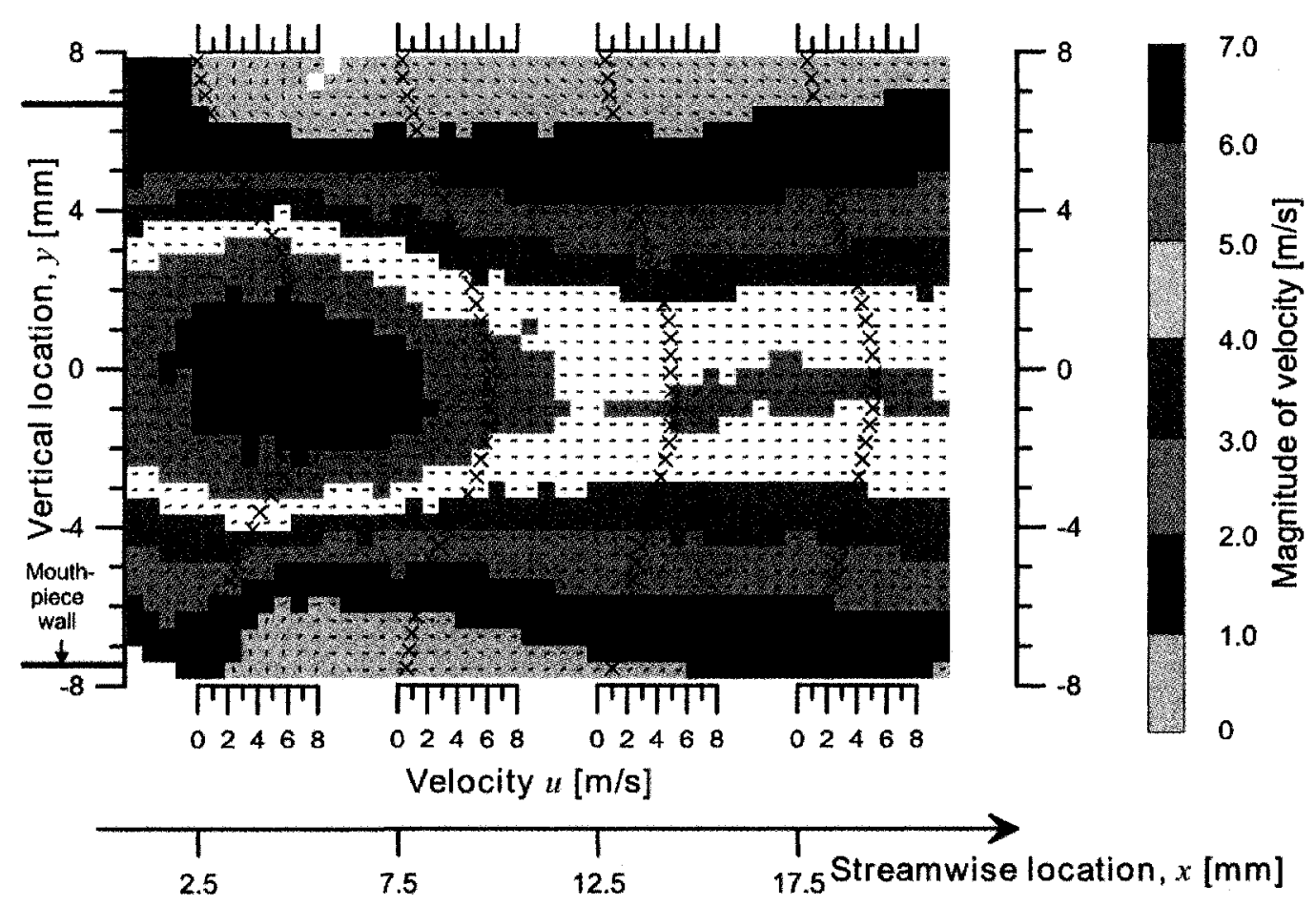

Figure D.3 - Average vector velocity flow field. Ventolin, spacer outlet, $5 \mathrm{~ms}$ 


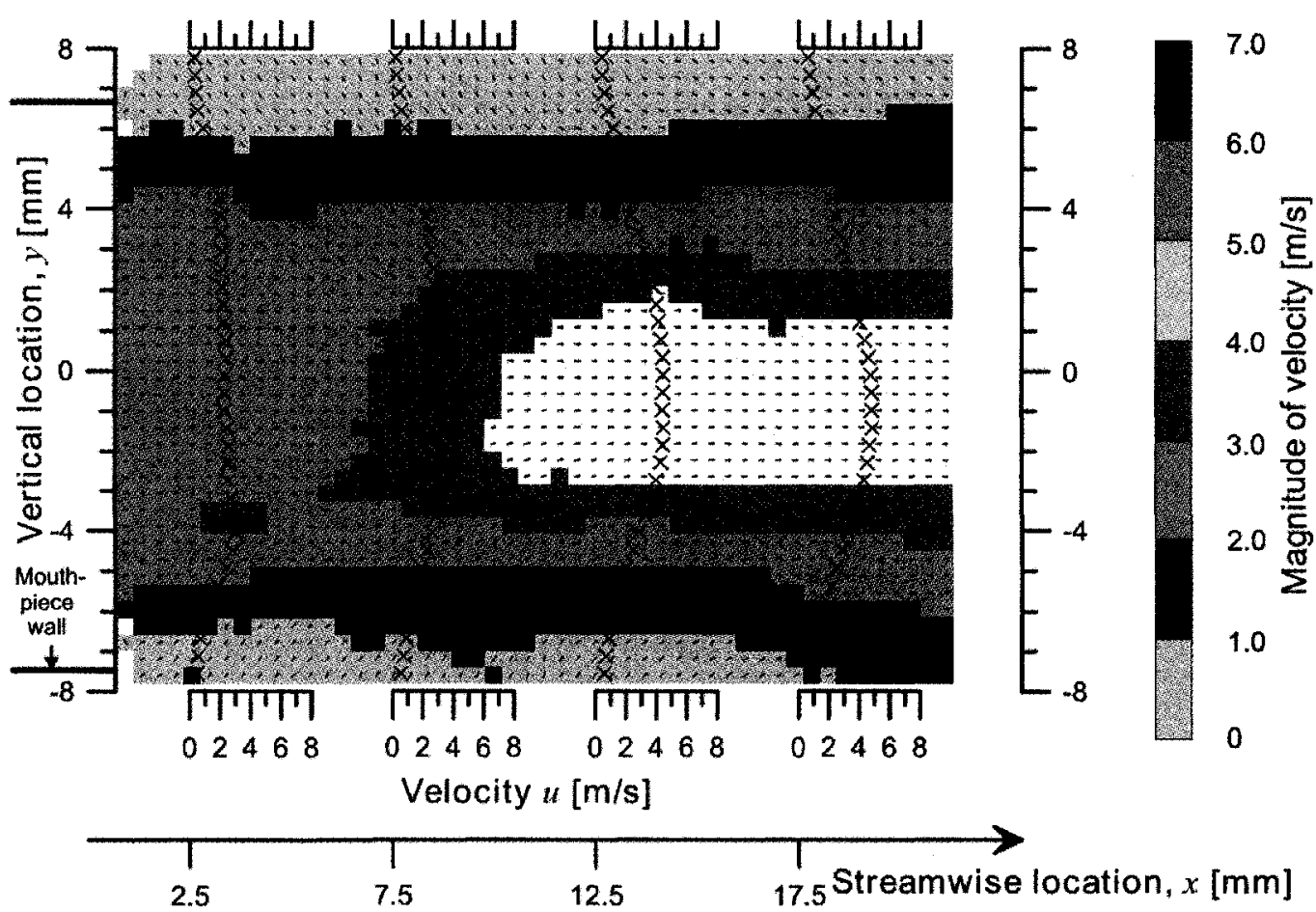

Figure D.4 - Average vector velocity flow field. Ventolin, spacer outlet, 7.5 ms.

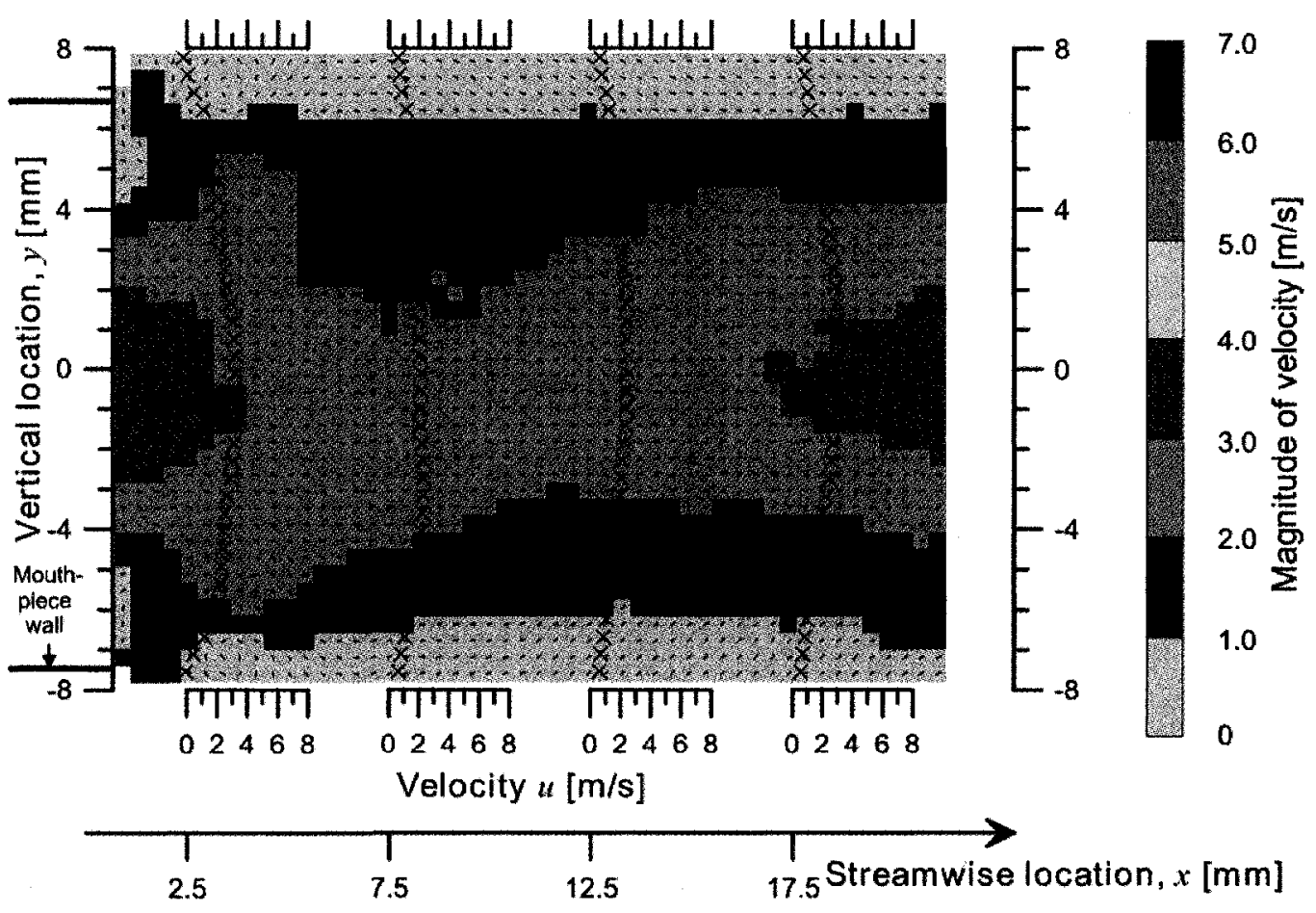

Figure D.5 - Average vector velocity flow field. Ventolin, spacer outlet, $10 \mathrm{~ms}$. 


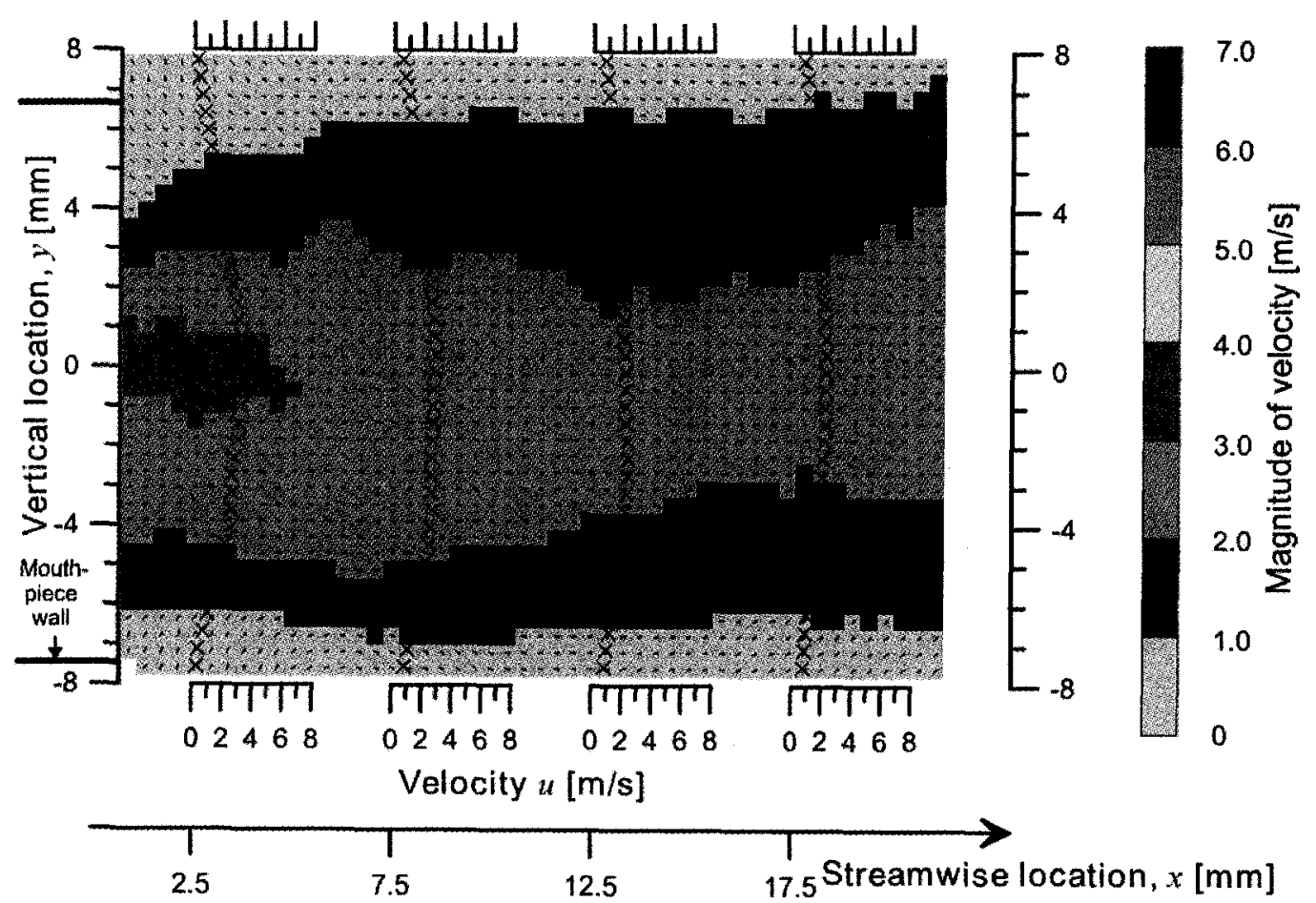

Figure D.6 - Average vector velocity flow field. Ventolin, spacer outlet, $12.5 \mathrm{~ms}$.

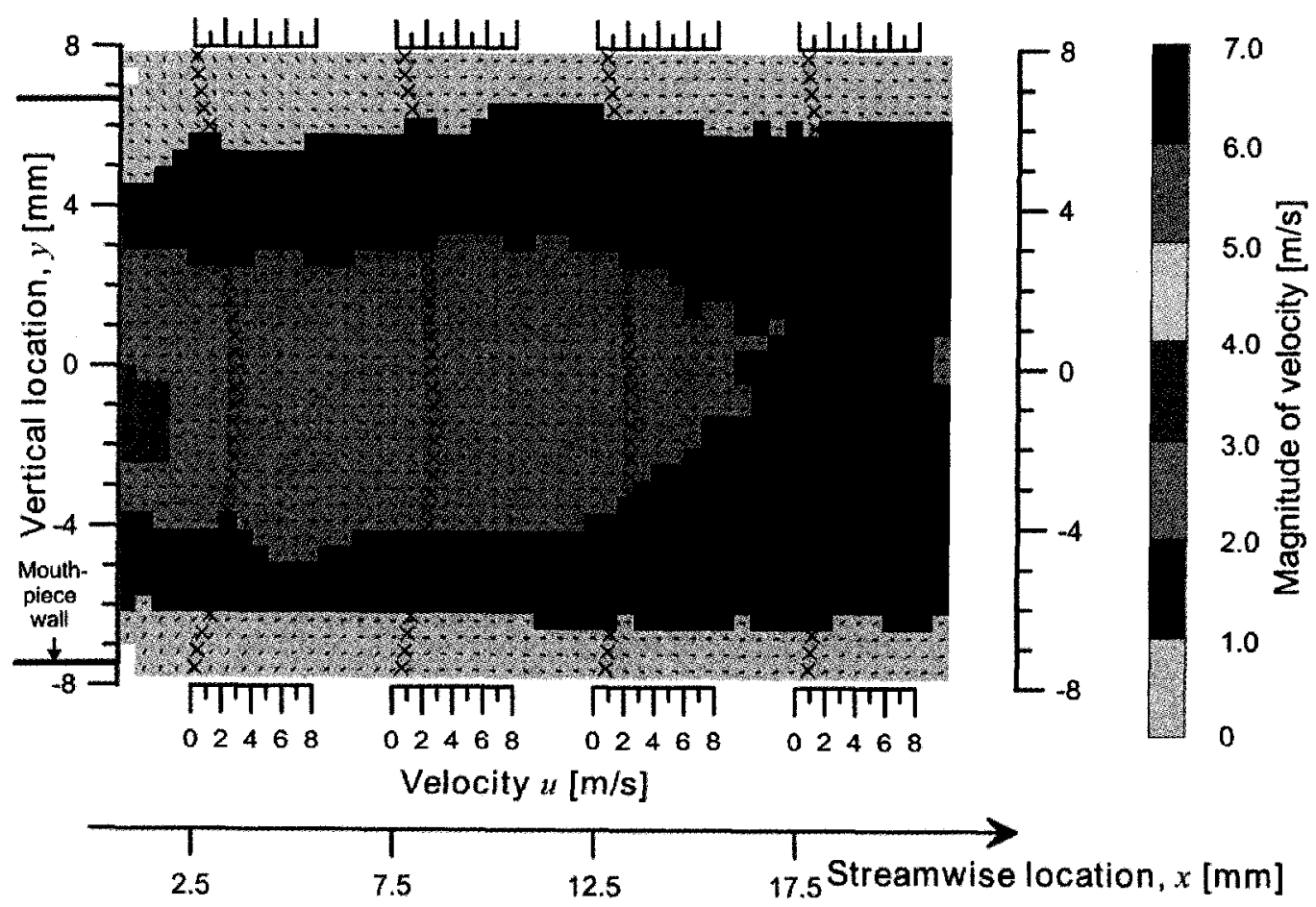

Figure D.7 - Average vector velocity flow field. Ventolin, spacer outlet, 15 ms. 


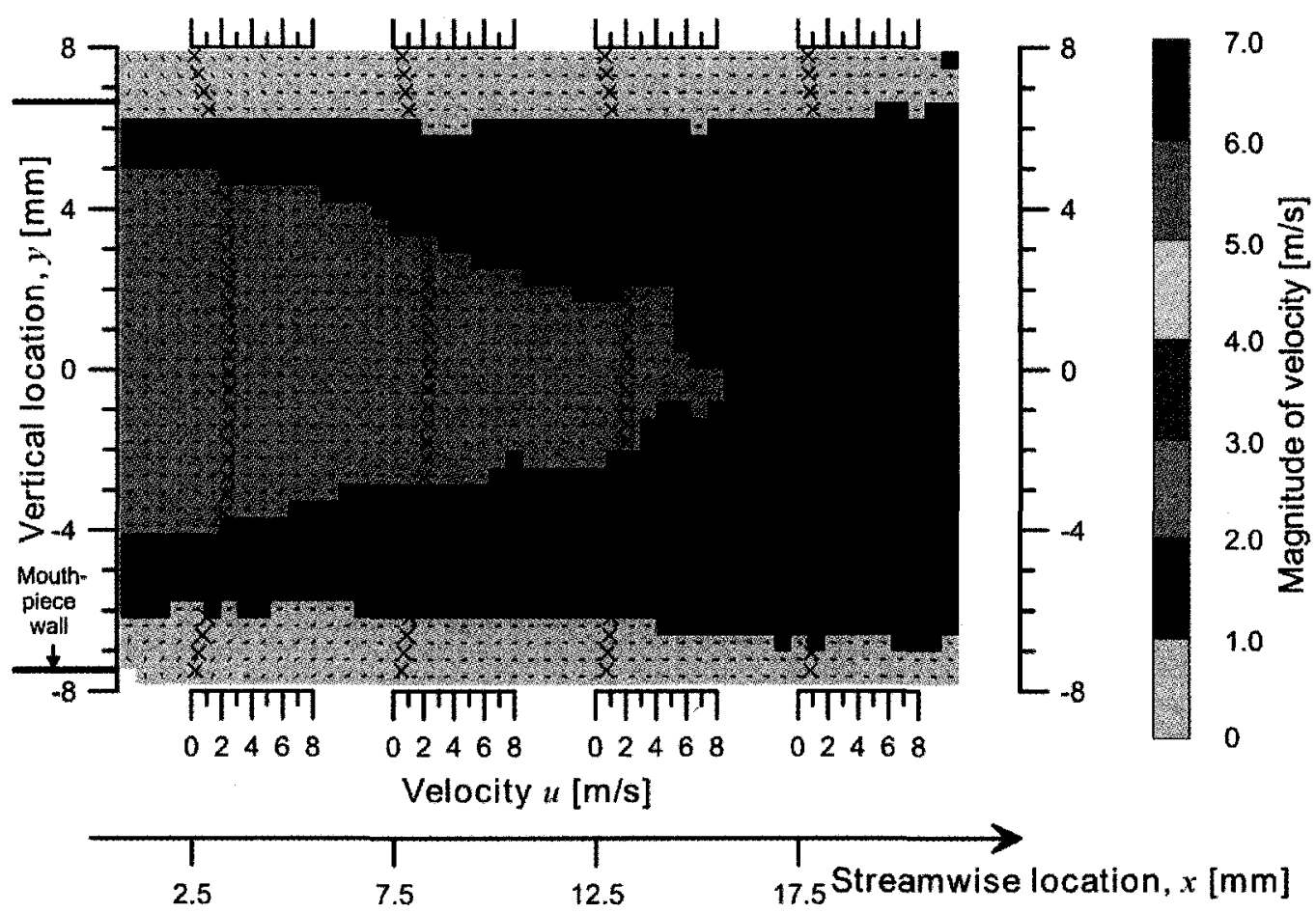

Figure D.8 - Average vector velocity flow field. Ventolin, spacer outlet, 20 ms.

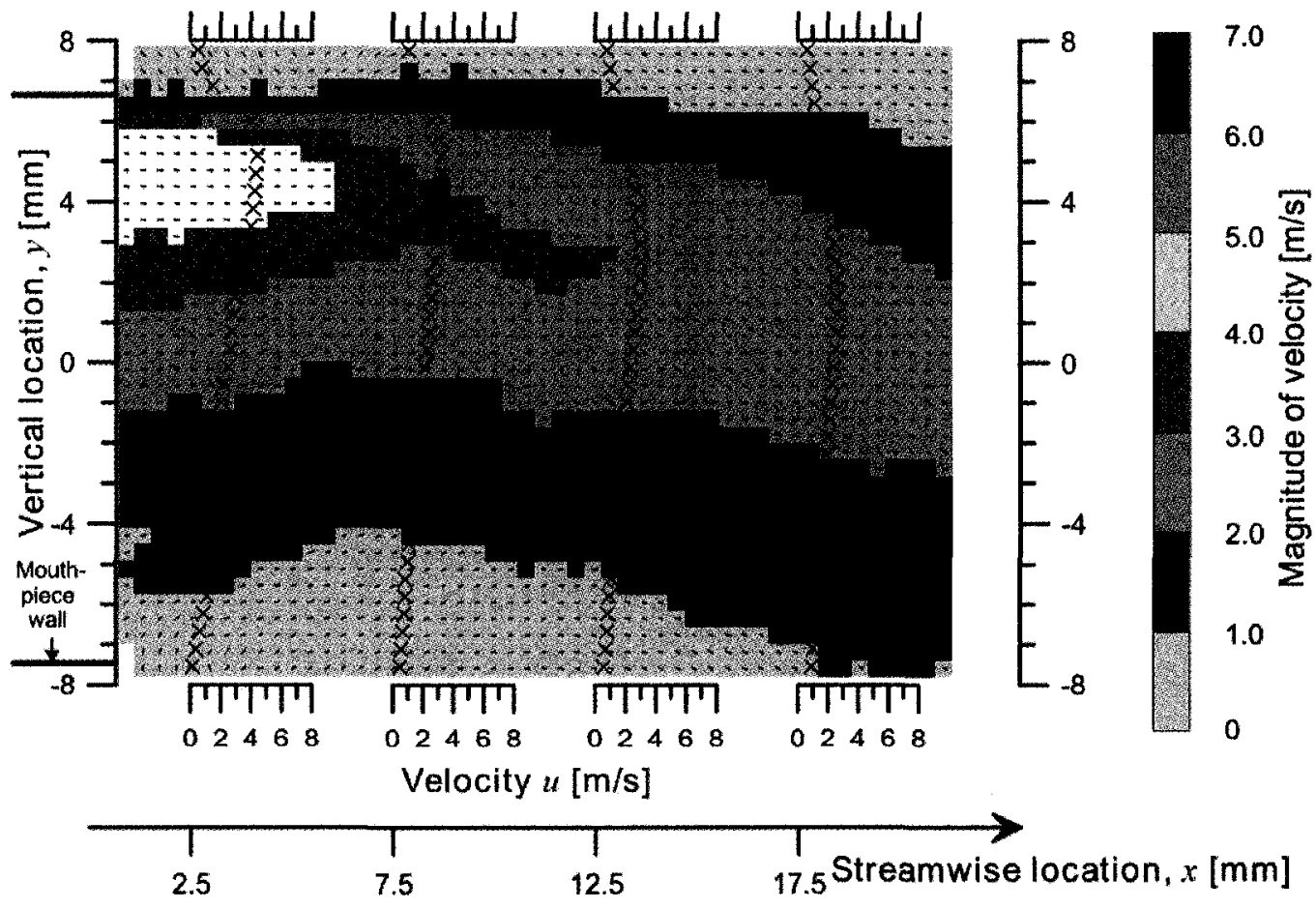

Figure D.9 - Average vector velocity flow field. Ventolin, spacer outlet, $40 \mathrm{~ms}$. 


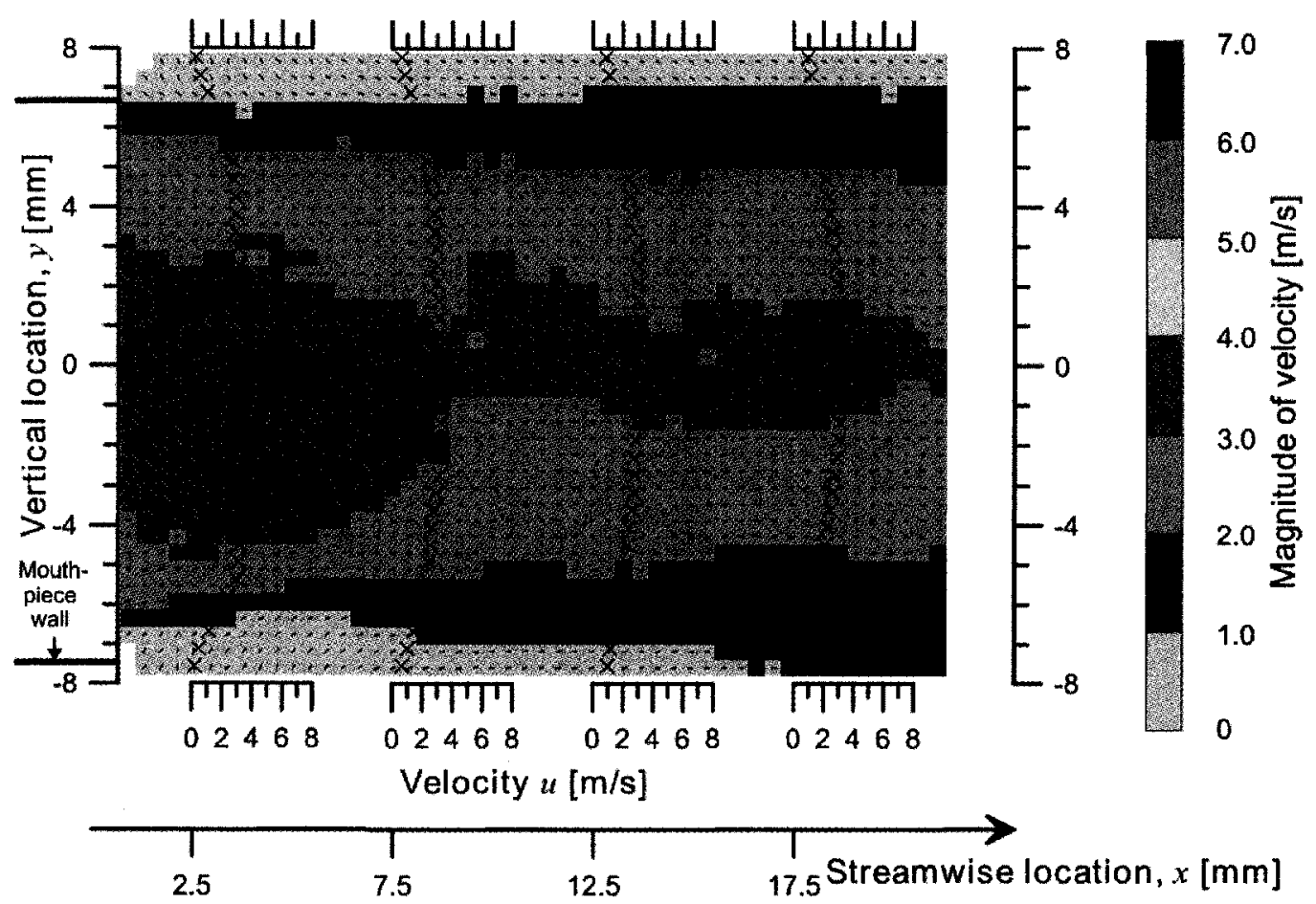

Figure D.10 - Average vector velocity flow field. Ventolin, spacer outlet, $60 \mathrm{~ms}$.

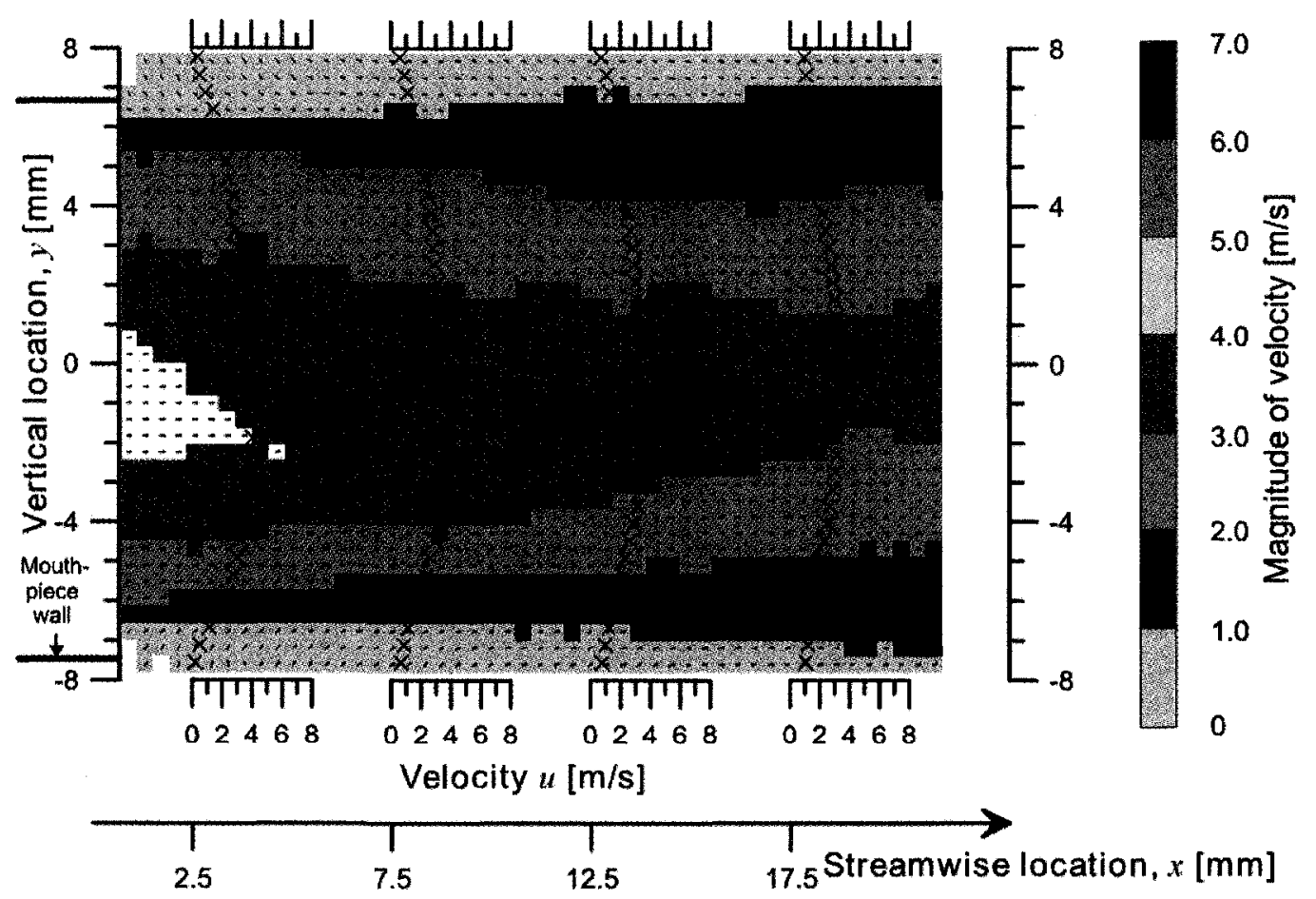

Figure D.11 - Average vector velocity flow field. Ventolin, spacer outlet, $100 \mathrm{~ms}$. 


\section{Appendix E: Average Turbulent Kinetic Energy}

As discussed in Section 5.5, the average turbulent kinetic energy, normalized by the local average velocity, can be calculated as follows:

$$
\frac{k_{\text {tke }}}{\bar{V}^{2}}=\frac{\overline{u^{\prime 2}}+\overline{2 v^{\prime 2}}}{2 \cdot \bar{V}^{2}}
$$

After additional post-processing (also discussed in Section 5.5) the application of equation 5.1 for ratiopharm canisters actuated into quiescent air for all time delays gives the average turbulent kinetic energy fields shown in Figures E.1 through E.10. The velocity measurements associated with this measurement condition are presented in Figures $4.4-4.8$; Table 5.3 presents average axial velocity and its standard deviation for four locations on the centreline of the spray. Note that the results are based on between 75-100 measurements, and as such are only an approximation of the true turbulent kinetic energies. 


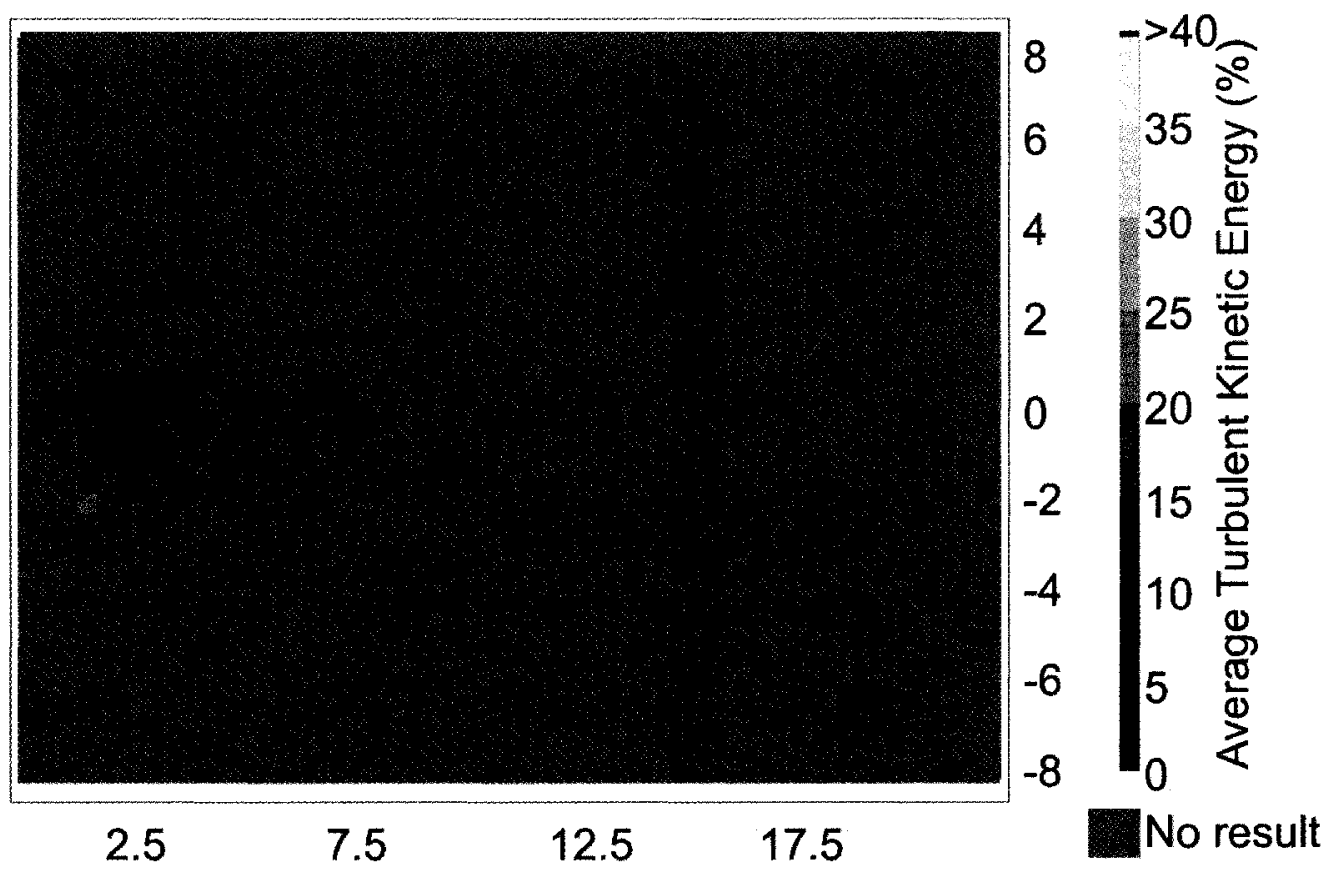

Figure E.1 - Turbulent kinetic energy. ratiopharm, $1.3 \mathrm{~ms}$.

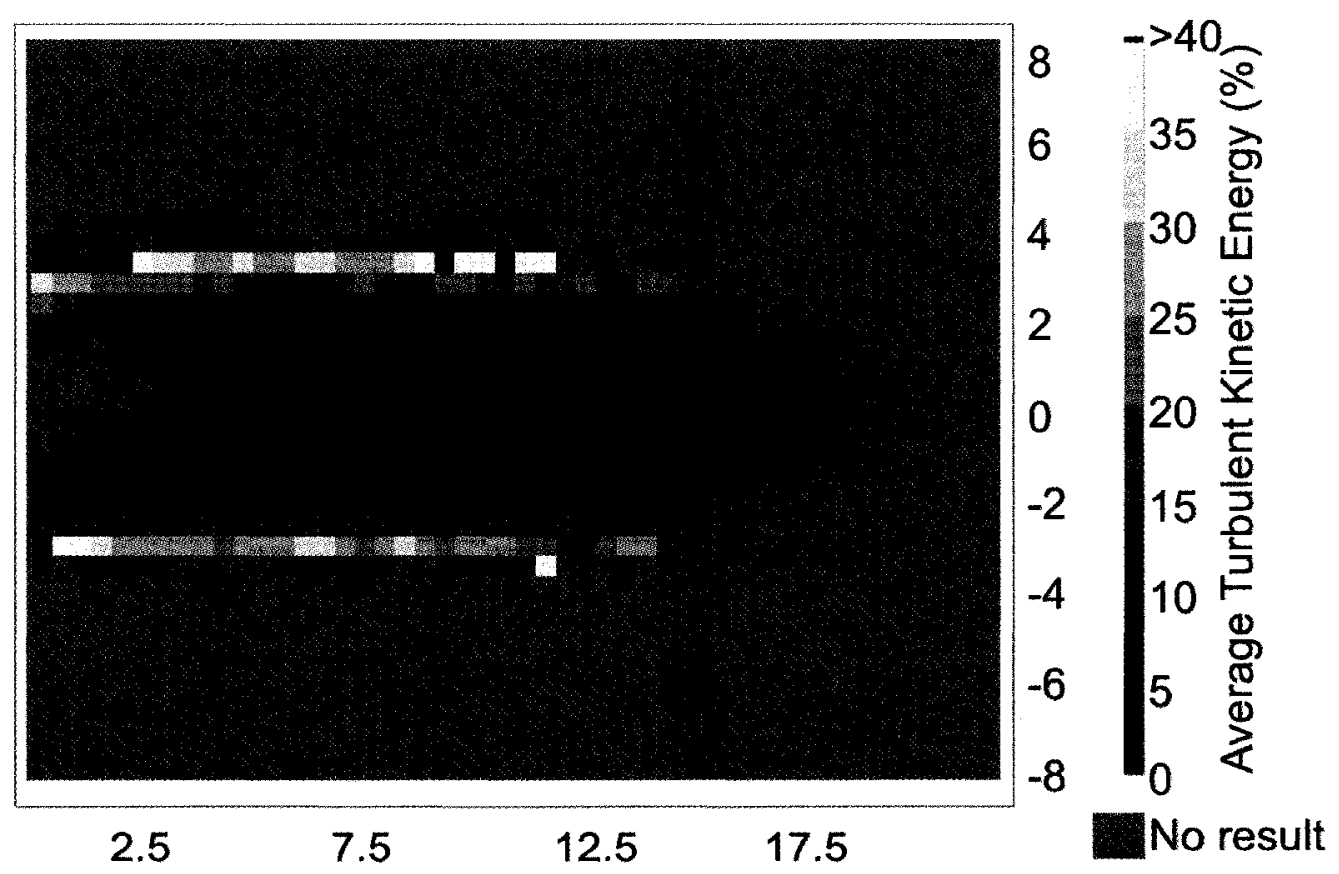

Figure E.2 - Turbulent kinetic energy. ratiopharm, $1.7 \mathrm{~ms}$. 


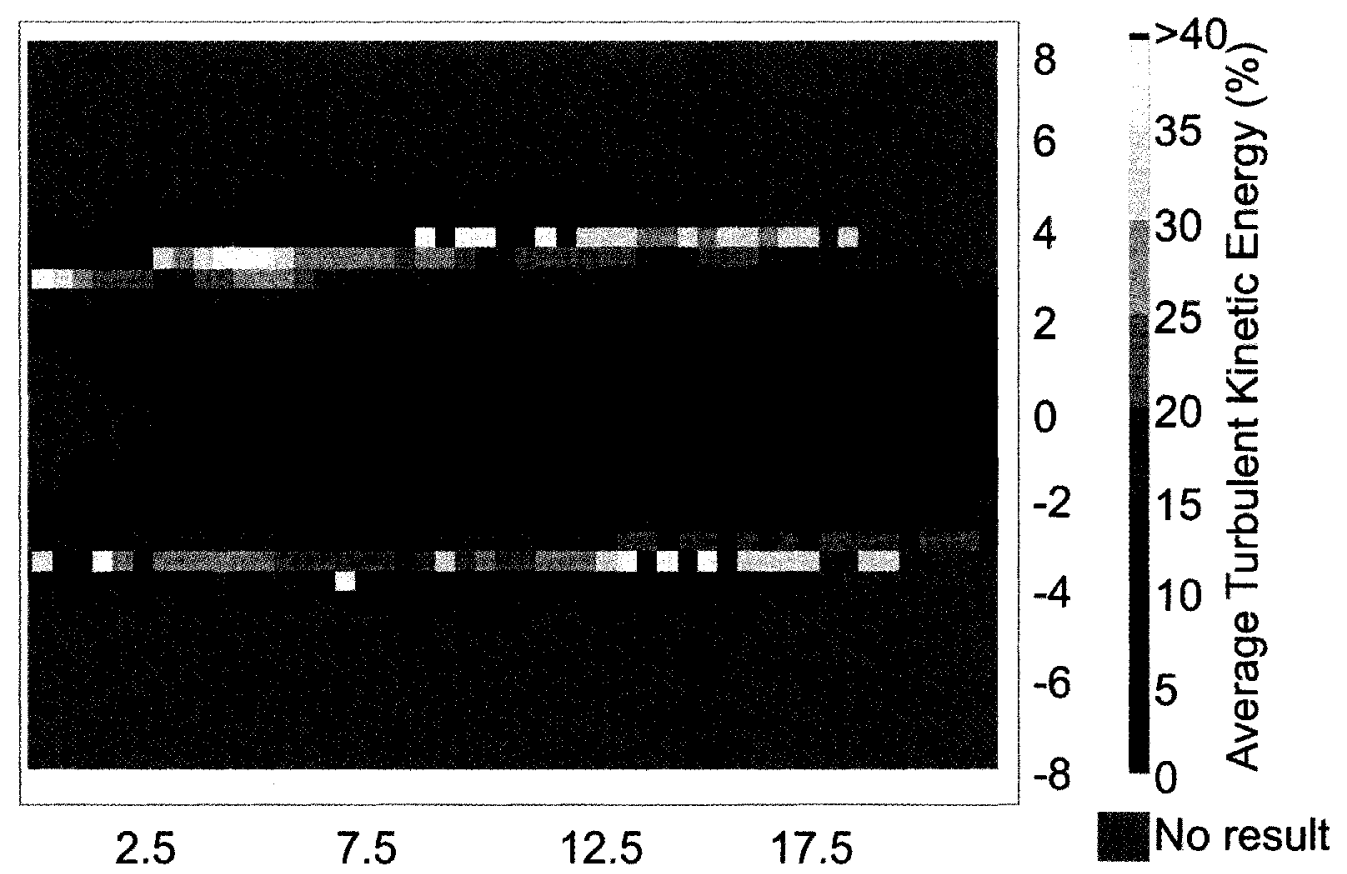

Figure E.3 - Turbulent kinetic energy. ratiopharm, 2 ms.

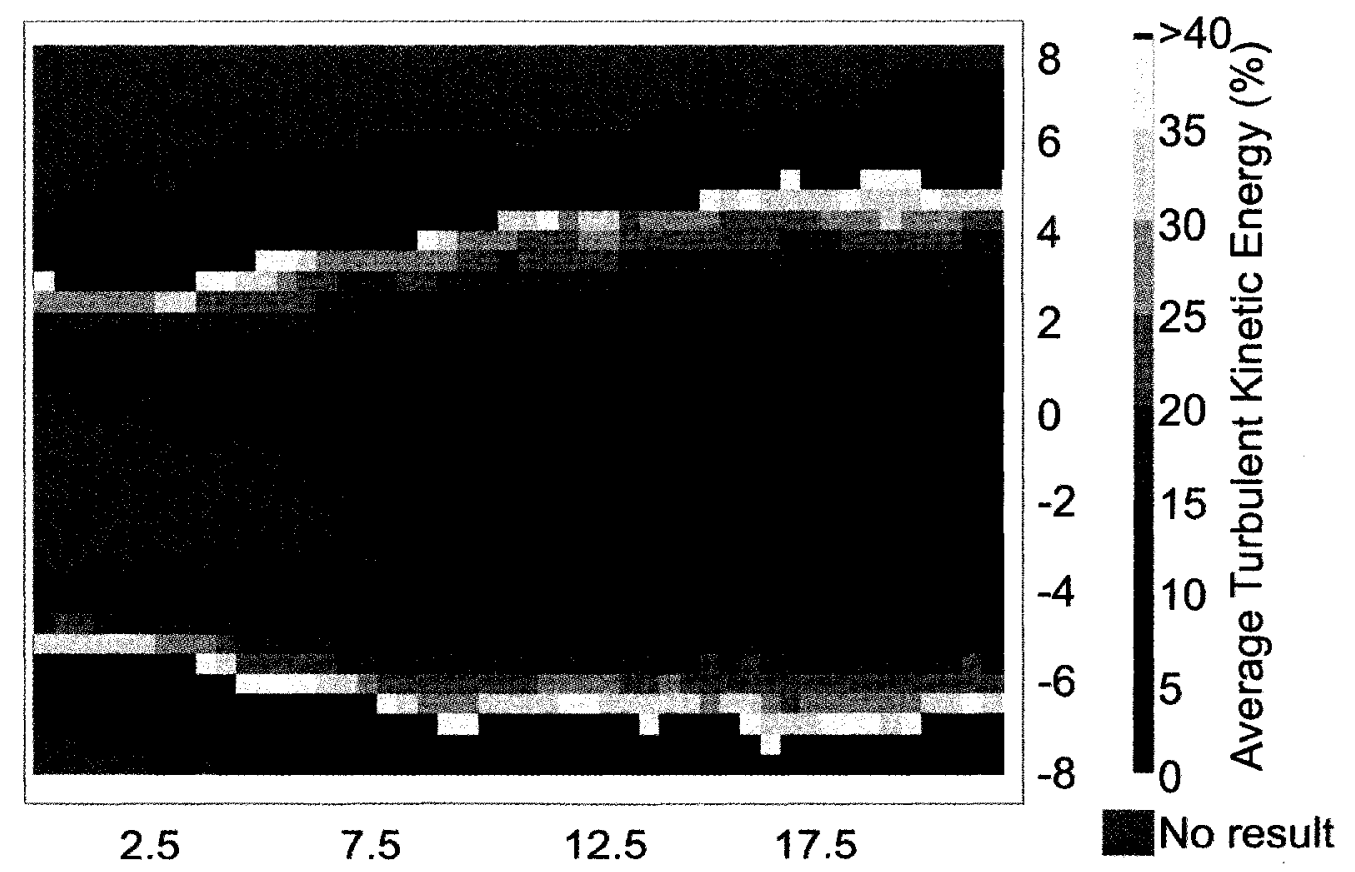

Figure E.4 - Turbulent kinetic energy. ratiopharm, 5 ms. 


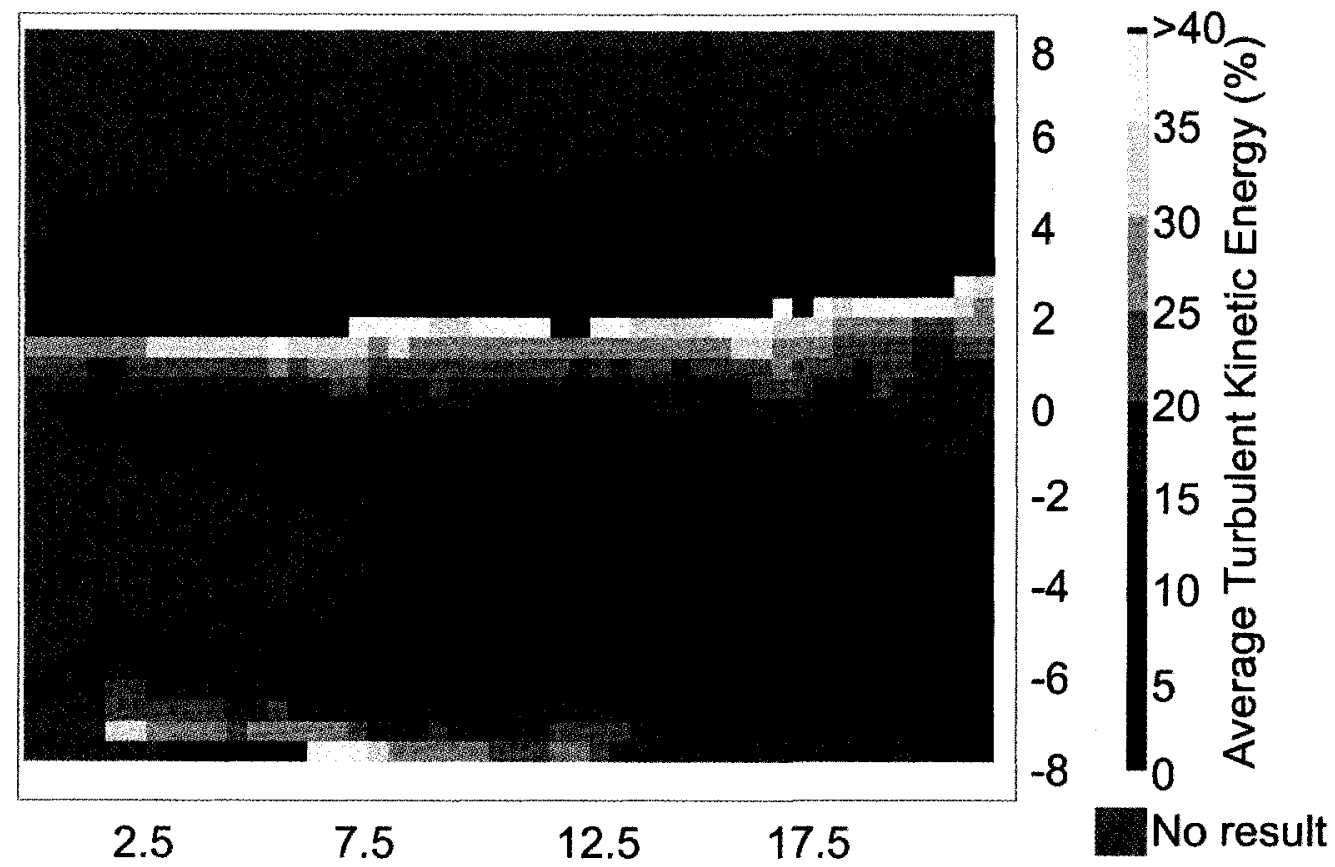

Figure E.5 - Turbulent kinetic energy. ratiopharm, $10 \mathrm{~ms}$.

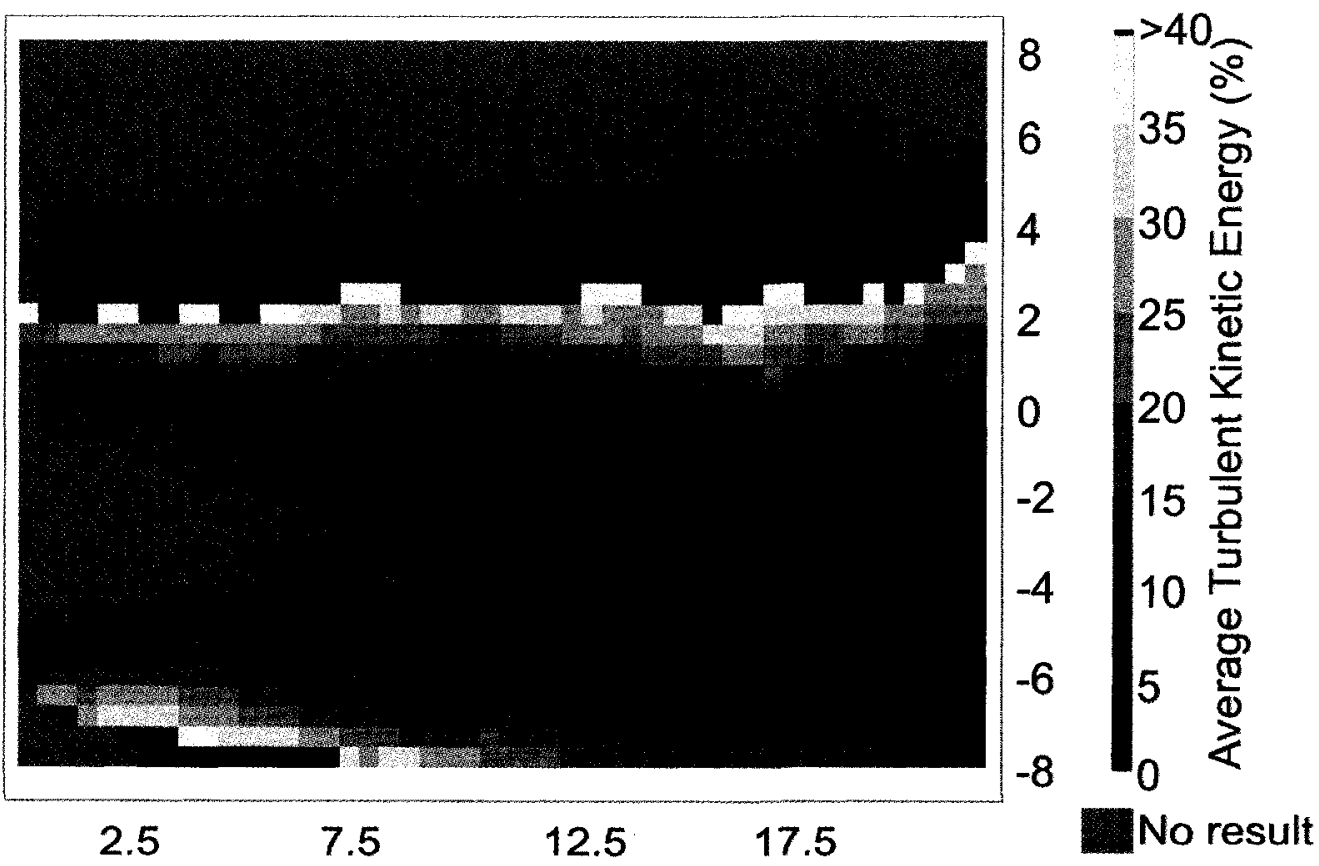

Figure E.6 - Turbulent kinetic energy. ratiopharm, 20 ms. 

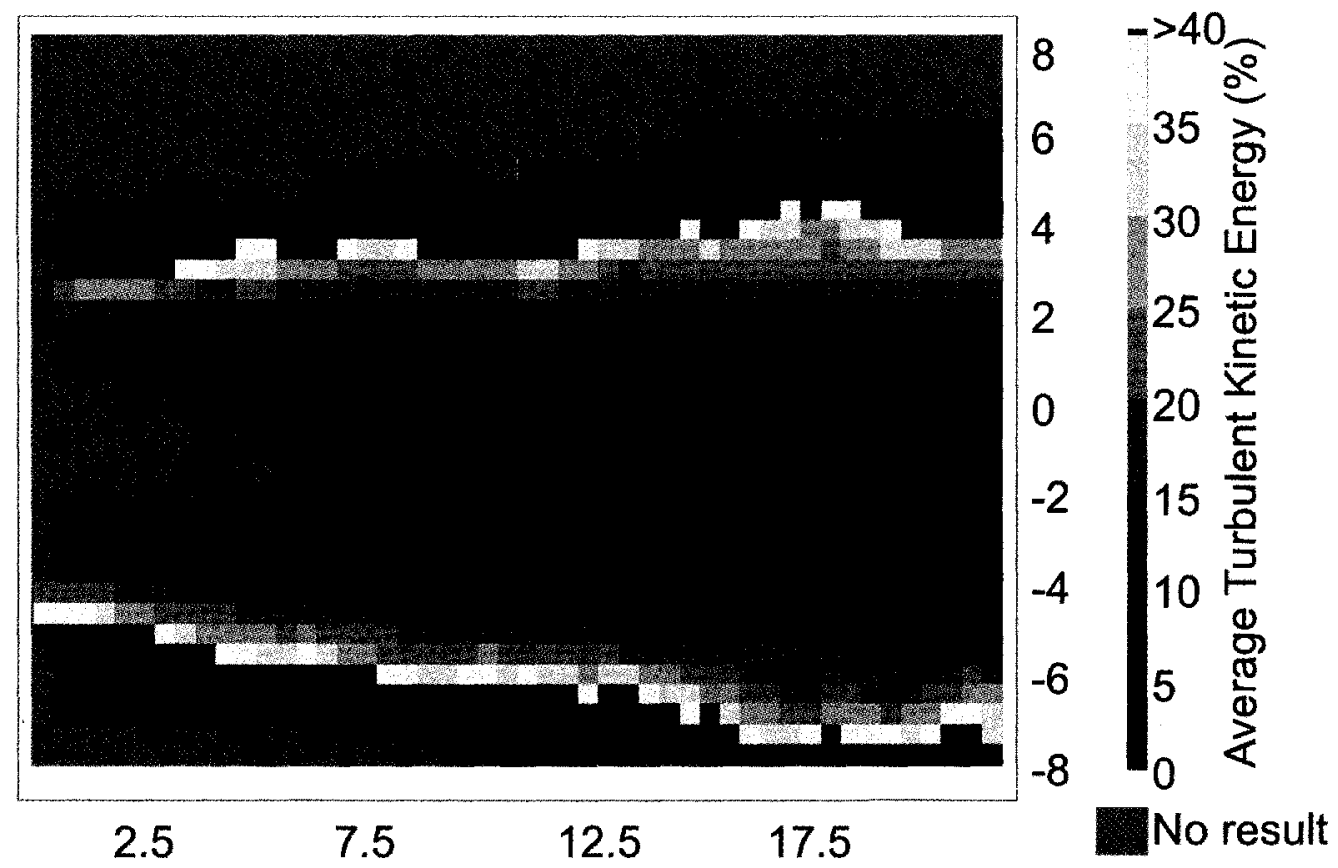

Figure E.7 - Turbulent kinetic energy. ratiopharm, 40 ms.

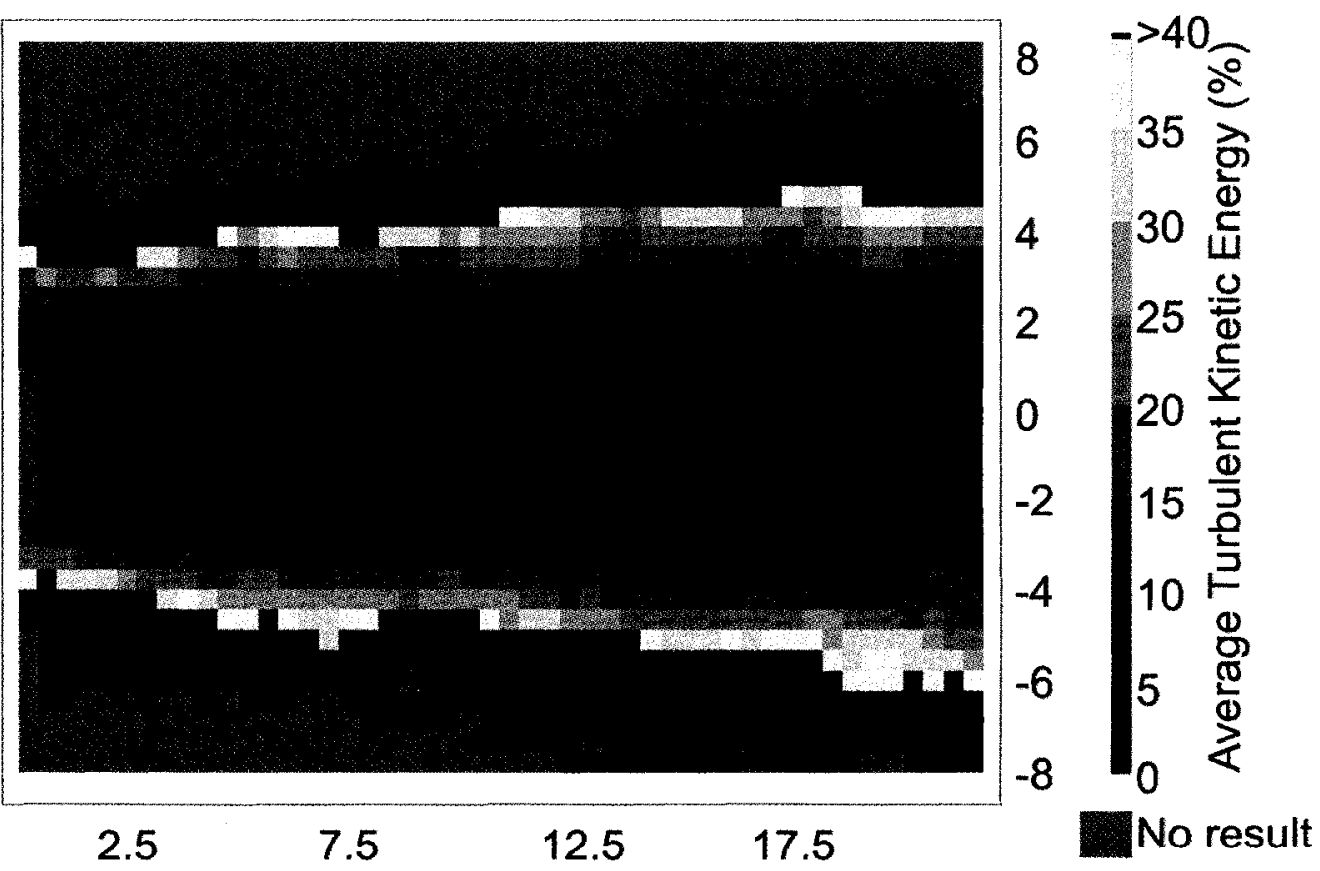

Figure E.8 - Turbulent kinetic energy. ratiopharm, $60 \mathrm{~ms}$. 


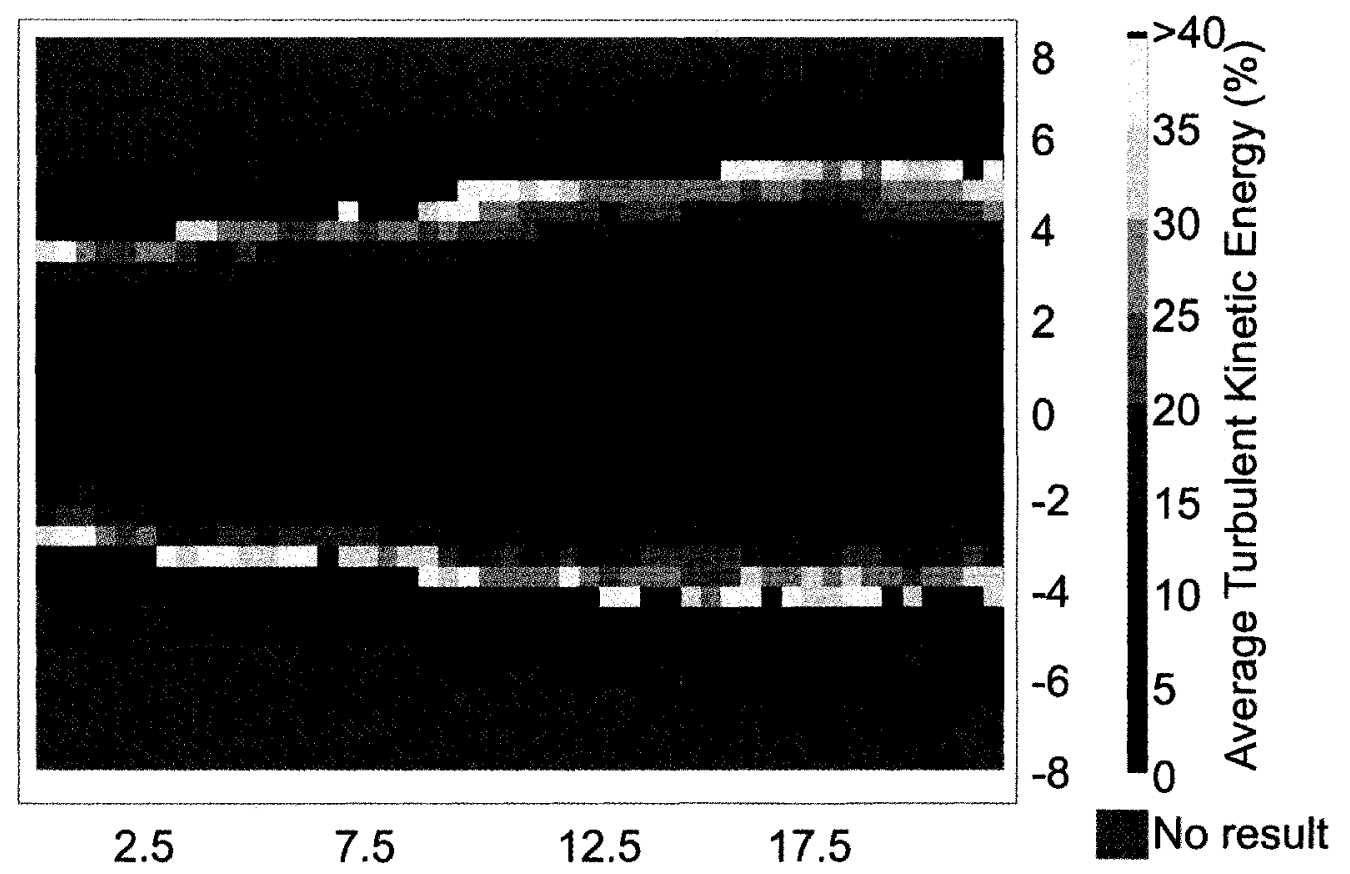

Figure E.9 - Turbulent kinetic energy. ratiopharm, 80 ms.

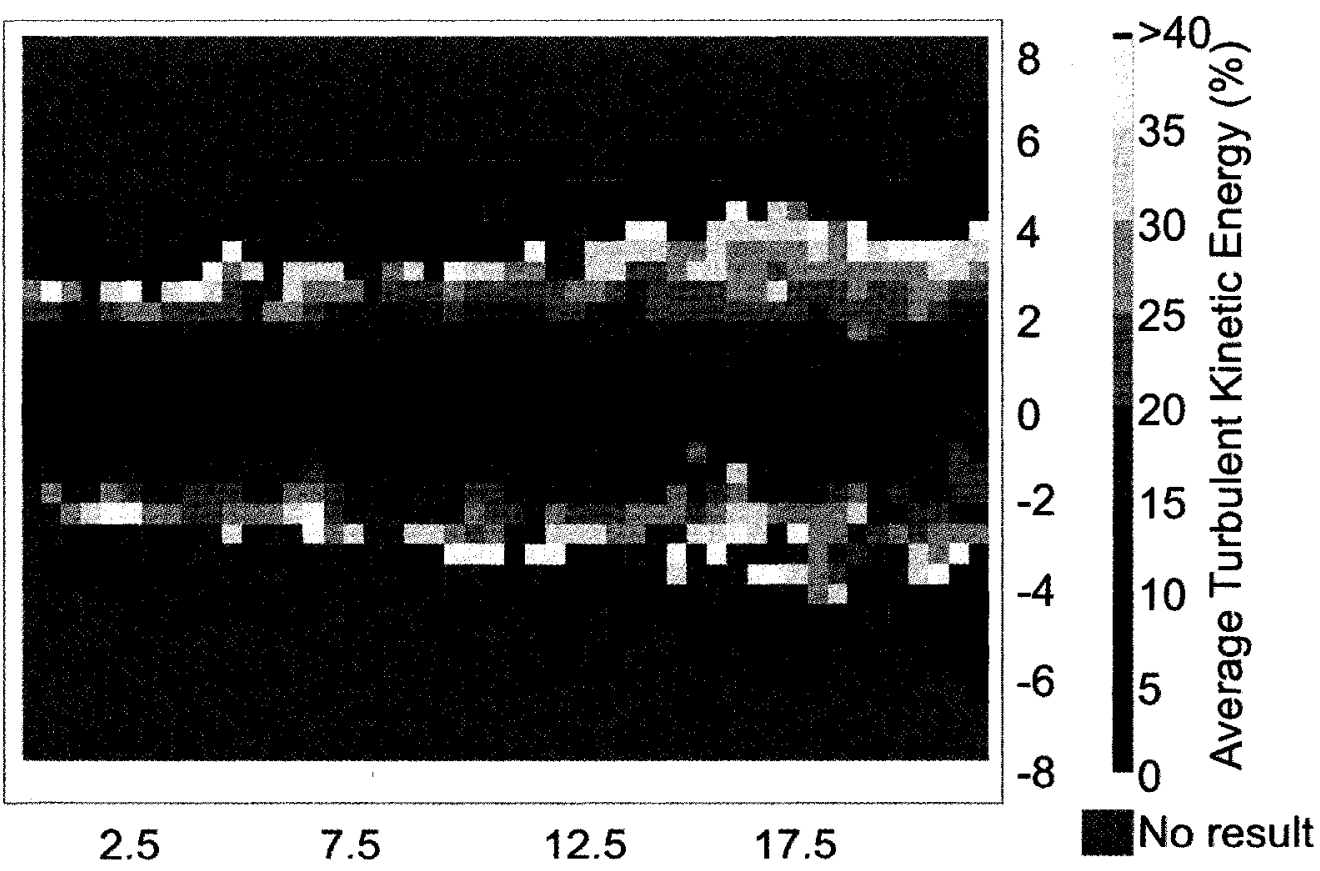

Figure E.10 - Turbulent kinetic energy. ratiopharm, $100 \mathrm{~ms}$. 


\section{Appendix F: Velocity Data Used for Curve Fits}

The data that were used to create the parametric curve fits described in Section 5.5 are presented here. Refer to Equation A.37 to calculate the measurement uncertainty associated with these measurements.

Table F.1 - Streamwise velocity at $x=1 \mathrm{~mm}$ for various vertical $(y)$ locations and time delays.

\begin{tabular}{|c|c|c|c|c|c|c|c|c|c|c|}
\hline & \multicolumn{10}{|c|}{ Time delay (ms) } \\
\hline & 1.3 & 1.7 & 2 & 5 & 10 & 20 & 40 & 60 & 80 & 100 \\
\hline $\begin{array}{l}\text { Vertical } \\
\text { location, } y\end{array}$ & \multicolumn{10}{|c|}{ Streamwise velocity, $u$} \\
\hline 8.192 & 0 & 0 & 0 & 0 & 0 & 0 & 0 & 0 & 0 & 0 \\
\hline 7.744 & 0 & 0 & 0 & 0 & 0 & 0 & 0 & 0 & 0 & 0 \\
\hline 7.296 & 0 & 0 & 0 & 0 & 0 & 0 & 0 & 0 & 0 & 0 \\
\hline 6.848 & 0 & 0 & 0 & 0 & 0 & 0 & 0 & 0 & 0 & 0 \\
\hline 6.400 & 0 & 0 & 0 & 0 & 0 & 0 & 0 & 0 & -1.75 & 0 \\
\hline 5.952 & 0 & 0 & 0 & -1.07 & -1.12 & 0 & 0 & -0.80 & -1.60 & 0 \\
\hline 5.504 & 0 & 0 & -2.99 & -0.30 & -1.50 & -1.28 & -0.10 & 0.04 & -0.75 & -0.27 \\
\hline 5.055 & 0 & -0.94 & -1.89 & -0.55 & -0.99 & 0.40 & -0.18 & 0.69 & 1.18 & 0.13 \\
\hline 4.607 & 0.56 & 0.26 & -0.99 & 0.32 & -0.54 & -0.86 & -0.13 & 1.11 & 2.00 & 0.94 \\
\hline 4.159 & 0.35 & 3.07 & 2.20 & 1.29 & -0.45 & -0.24 & 0.48 & 1.83 & 2.92 & 2.15 \\
\hline 3.711 & 3.34 & 7.23 & 4.47 & 2.71 & 0.89 & 0.51 & 1.38 & 3.31 & 4.46 & 2.45 \\
\hline 3.263 & 8.32 & 11.90 & 9.20 & 4.86 & 2.23 & 1.61 & 3.62 & 6.22 & 6.76 & 3.34 \\
\hline 2.815 & 14.91 & 17.89 & 14.69 & 7.33 & 3.84 & 3.53 & 6.92 & 9.73 & 9.98 & 5.48 \\
\hline 2.367 & 22.95 & 23.56 & 20.35 & 10.68 & 5.42 & 6.26 & 10.64 & 13.95 & 13.86 & 8.47 \\
\hline 1.919 & 32.85 & 31.01 & 27.02 & 14.12 & 7.90 & 10.13 & 15.04 & 18.51 & 18.78 & 11.41 \\
\hline 1.470 & 43.84 & 40.20 & 34.17 & 17.49 & 10.75 & 14.52 & 21.46 & 24.40 & 24.78 & 14.34 \\
\hline
\end{tabular}




$\begin{array}{rrrrrrrrrrr}1.022 & 52.19 & 48.44 & 42.23 & 21.25 & 13.97 & 18.69 & 28.51 & 32.18 & 30.11 & 16.85 \\ 0.574 & 59.89 & 55.59 & 49.96 & 25.07 & 17.20 & 22.72 & 35.29 & 38.97 & 34.45 & 19.28 \\ 0.126 & 62.51 & 60.00 & 57.34 & 28.70 & 20.65 & 26.72 & 40.85 & 43.66 & 35.70 & 21.08 \\ -0.322 & 59.79 & 59.38 & 60.58 & 32.31 & 23.67 & 31.20 & 44.20 & 45.52 & 33.06 & 21.21 \\ -0.770 & 54.48 & 56.27 & 59.30 & 34.51 & 26.36 & 34.81 & 44.96 & 43.81 & 28.87 & 19.41 \\ -1.218 & 46.35 & 49.70 & 53.79 & 35.30 & 28.12 & 37.20 & 43.34 & 38.22 & 24.10 & 16.87 \\ -1.667 & 36.38 & 41.11 & 45.63 & 35.58 & 29.11 & 37.90 & 39.40 & 29.69 & 18.21 & 13.45 \\ -2.115 & 25.38 & 32.63 & 36.68 & 34.59 & 30.72 & 37.31 & 35.05 & 21.79 & 12.99 & 10.30 \\ -2.563 & 17.71 & 23.58 & 28.57 & 33.05 & 30.73 & 36.30 & 29.40 & 15.95 & 9.42 & 8.90 \\ -3.011 & 10.20 & 15.60 & 19.52 & 30.56 & 29.48 & 33.31 & 23.57 & 11.41 & 6.47 & 6.82 \\ -3.459 & 4.30 & 8.91 & 12.26 & 26.47 & 28.21 & 29.78 & 18.58 & 7.77 & 3.70 & 4.31 \\ -3.907 & 0.05 & 4.82 & 7.22 & 22.25 & 26.50 & 25.51 & 14.53 & 5.27 & 2.05 & 2.91 \\ -4.355 & 0.27 & 2.55 & 3.86 & 18.61 & 23.50 & 21.87 & 10.70 & 2.82 & 1.78 & 1.83 \\ -4.803 & 0 & -1.12 & 1.76 & 14.49 & 20.03 & 19.42 & 7.37 & 1.33 & 0.56 & 0.81 \\ -5.252 & 0 & 0 & 0 & 10.95 & 17.43 & 16.91 & 4.90 & 1.07 & 0.11 & 0.89 \\ -5.700 & 0 & 0 & 0 & 8.11 & 15.15 & 14.58 & 3.14 & 1.38 & 0.51 & -0.60 \\ -6.148 & 0 & 0 & 0 & 5.59 & 13.24 & 12.00 & 2.35 & 1.33 & -0.06 & -0.32 \\ -6.596 & 0 & 0 & 0 & 4.01 & 11.40 & 9.59 & 2.14 & 1.27 & 0.46 & -0.71 \\ -7.044 & 0 & 0 & 0 & 3.10 & 8.93 & 7.11 & 1.99 & 2.00 & 3.15 & 2.40 \\ -7.492 & 0 & 0 & 0 & 2.53 & 6.03 & 4.54 & 1.18 & 1.68 & 0 & 0 \\ -7.940 & 0 & 0 & 0 & 2.16 & 3.66 & 3.08 & 0 & 1.39 & 0 & 0 \\ -8.388 & 0 & 0 & 0 & 0 & 0 & 0 & 0 & 0 & 0 & 0\end{array}$

Table F.1 - Vertical velocity at $x=1 \mathrm{~mm}$ for various vertical $(y)$ locations and time delays.

\begin{tabular}{rrrrrrrrrrrr}
\hline \multicolumn{10}{c}{ Time delay (ms) } \\
\hline \hline $\begin{array}{c}\text { Vertical } \\
\text { location, } y\end{array}$ & 1.3 & 1.7 & 2 & \multicolumn{10}{c}{$\begin{array}{c}10 \\
\text { Vertical velocity, } v\end{array}$} \\
\hline 8.192 & 0 & 0 & 0 & 0 & 0 & 0 & 0 & 0 & 0 & 0 \\
7.744 & 0 & 0 & 0 & 0 & 0 & 0 & 0 & 0 & 0 & 0 \\
7.296 & 0 & 0 & 0 & 0 & 0 & 0 & 0 & 0 & 0 & 0 \\
6.848 & 0 & 0 & 0 & 0 & 0 & 0 & 0 & 0 & 0 & 0 \\
6.400 & 0 & 0 & 0 & 0 & 0 & 0 & 0 & -0.62 & 0 & 0 \\
5.952 & 0 & 0 & -1.40 & -0.49 & 0 & 0 & 0.08 & 0.62 & 0 & 0
\end{tabular}




\begin{tabular}{|c|c|c|c|c|c|c|c|c|c|c|}
\hline 5.504 & 0 & 1.14 & -0.07 & -0.22 & 0.19 & -0.18 & 0.92 & 0.17 & 0.67 & 0 \\
\hline .055 & .29 & 0.51 & -0.33 & 0.29 & 0.14 & 0.11 & 1.54 & 0.30 & 1.33 & -0.29 \\
\hline 4.607 & 0.22 & -0.23 & 0.31 & 0.40 & -0.17 & 0.53 & 1.37 & .14 & .26 & 0.22 \\
\hline 159 & 0.88 & .29 & 0.25 & .14 & -0.21 & 0.40 & .49 & 2.05 & 1.14 & 0.88 \\
\hline 3.711 & 0.19 & 0.14 & 0.44 & 0.51 & 0.23 & 0.64 & 1.74 & 2.33 & 1.25 & 0.19 \\
\hline 3.263 & 0.56 & 0.51 & 0.79 & 0.86 & 0.64 & 0.96 & 1.46 & 2.04 & 1.55 & 0.56 \\
\hline 2.815 & 0.89 & 1.18 & 0.92 & 1.10 & 0.94 & 0.88 & 1.48 & 2.40 & 1.96 & 0.89 \\
\hline 2.367 & 1.07 & 1.67 & 0.88 & 0.71 & 1.14 & 0.74 & .88 & 2.34 & .67 & 1.07 \\
\hline 1.919 & 1.68 & 1.81 & 0.82 & 0.46 & 0.93 & 0.70 & 1.53 & 1.69 & 1.81 & 1.68 \\
\hline 1.470 & 2.33 & 2.26 & 0.63 & 0.24 & 0.87 & 0.71 & 0.77 & 1.06 & 1.53 & 2.33 \\
\hline 1.022 & 2.29 & 1.91 & 0.19 & -0.06 & 0.52 & 0.61 & 0.65 & 0.82 & 0.95 & 2.29 \\
\hline .574 & 1.56 & 1.10 & -0.31 & -0.35 & 0.09 & 0.16 & 0.40 & 0.50 & 0.60 & 1.56 \\
\hline 0.126 & 1.15 & 0.05 & -0.75 & -0.81 & -0.73 & -0.22 & -0.29 & 0.00 & 0.33 & 1.15 \\
\hline-0.322 & 0.10 & -0.84 & -1.12 & -1.42 & -1.40 & -0.81 & -0.95 & -0.42 & 0.30 & 0.10 \\
\hline-0.770 & .95 & -1.69 & -2.0 & ... & -2.23 & -1.71 & . & -0.42 & 0.21 & -0.95 \\
\hline 1.218 & -1.65 & -2.38 & -2.83 & -1.94 & -3.04 & -2.33 & -1.84 & -0.29 & 0.02 & -1.65 \\
\hline-1.667 & -2.29 & -2.79 & -3.28 & -2.60 & -3.44 & -2.63 & -1.77 & -0.03 & -0.10 & -2.29 \\
\hline-2.115 & -2.73 & -2.81 & -2.96 & -3.41 & -4.15 & -2.87 & -1.08 & 0.17 & 0.37 & -2.73 \\
\hline-2.563 & -2.51 & -2.65 & -3.08 & -3.9 & -4.30 & -2.41 & -0.30 & 0.84 & 0.27 & -2.51 \\
\hline-3.011 & -1.80 & -1.70 & -3.20 & -4.05 & -4.17 & -2.34 & 0.48 & 1.78 & 0.87 & -1.80 \\
\hline-3.459 & -1.54 & -0.69 & -2.98 & -3.98 & -4.32 & -2.03 & 1.19 & 2.80 & 2.12 & -1.54 \\
\hline-3.907 & -0.62 & 0.08 & -3.06 & -3.60 & -3.93 & -1.41 & 1.58 & 2.61 & 2.17 & -0.62 \\
\hline 4055 & -0.26 & -0.09 & -2 & -3 & -3.48 & -0.82 & 1.50 & 2.86 & 1.72 & -0.26 \\
\hline-4.803 & 0.62 & -0.02 & -2.62 & -2.77 & -3.08 & -0.45 & 2.12 & 2.77 & 1.59 & 0.62 \\
\hline-5.252 & 0 & 0 & -2.10 & -2.67 & -2.36 & -0.08 & 2.32 & 1.90 & 1.39 & 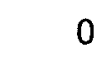 \\
\hline-5.700 & 0 & 0 & -1.35 & -2.48 & -1.77 & 0.41 & 2.07 & 2.19 & 1.45 & 0 \\
\hline-6.148 & 0 & 0 & -0.58 & -2.17 & -1.19 & 0.68 & 2.09 & 1.87 & 2.09 & 0 \\
\hline-6.596 & 0 & 0 & -0.21 & -1.51 & -0.87 & 0.59 & 1.68 & 1.34 & 1.04 & 0 \\
\hline-7.044 & 0 & 0 & -0.16 & -1.06 & -0.74 & 0.72 & 1.66 & 1.19 & 0.85 & 0 \\
\hline-7.492 & 0 & 0 & 0.05 & -1.06 & -0.34 & 0.01 & 1.09 & 0 & 0 & 0 \\
\hline-7.940 & 0 & 0 & 0.07 & -0.66 & -0.63 & 0 & 0.29 & 0 & 0 & 0 \\
\hline-8.388 & 0 & 0 & 0 & 0 & 0 & 0 & 0 & 0 & 0 & 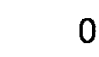 \\
\hline
\end{tabular}




\section{References}

Barry, P. W. and C. O'Callaghan (1999). "A comparative analysis of the particle size output of beclomethasone diproprionate, salmeterol xinafoate and fluticasone propionate metered dose inhalers used with the Babyhaler, Volumatic and Aerochamber spacer devices." British Journal of Clinical Pharmacology 47(4): 357360 .

Barry, P. W. and C. O'Callaghan (1995a). "The optimum size and shape of spacer devices for inhalational therapy." Journal of Aerosol Medicine-Deposition Clearance and Effects in the Lung 8(3): 303-305.

Barry, P. W. and C. O'Callaghan (1995b). "The effect of delay, multiple actuations and spacer static charge on the in-vitro delivery of budesonide from the nebuhaler." British Journal of Clinical Pharmacology 40(1): 76-78.

Bates, D.V., B.R. Fish et al. (1966). "Deposition and retention models for internal dosimetry of the human respiratory tract." Health Physics 12(2): 173-207.

Berg, E., J. Madsen, et al. (1998). "In vitro performance of three combinations of spacers and pressurized metered dose inhalers for treatment in children." European Respiratory Journal 12(2): 472-476.

Boillot, A. and A.K. Prasad. (1996). "Optimization procedure for pulse separation in cross-correlation PIV.” Experiments in Fluids 21(2): 87-93.

Cheng, Y.S. (2003). "Aerosol deposition in the extrathoracic region." Aerosol Science and Technology 37(8): 659-671.

Cheng, Y. S., C. S. Fu, et al. (2001). "Respiratory deposition patterns of salbutamol pMDI with CFC and HFA-134a formulations in a human airway replica." Journal of Aerosol Medicine-Deposition Clearance and Effects in the Lung 14(2): 255-266.

Clark, A.R. (1991). "Metered atomization for respiratory drug delivery." $\mathrm{PhD}$ thesis. Loughborough University of Technology. Loughborough, UK. 
Clarke, J.G., S.J. Farr et al. (1992). “TC-99M labeling of suspension type pressurized metered dose inhalers comprising various drug surfactant combinations." International Journal of Pharmaceutics 80(1):R1-R5.

Dunbar, C. A., A. P. Watkins, et al. (1997a). "An experimental investigation of the spray issued from a pMDI using laser diagnostic techniques." Journal of Aerosol MedicineDeposition Clearance and Effects in the Lung 10(4): 351-368.

Dunbar, C. A., A. P. Watkins, et al. (1997b). "Theoretical investigation of the spray from a pressurized metered-dose inhaler.” Atomization and Sprays 7(4): 417-436.

Dunbar, C. A. (1996). "Experimental and theoretical investigation of the spray issued from a pressurised metered-dose inhaler." $\mathrm{PhD}$ thesis. University of Manchester. Manchester, UK.

Echols, W. H. and J. A. Young (1963). "Studies of Portable Air-Operated Aerosol Generators.” US Naval Research Laboratory Report 5929.

Fadl, A.J., J. Wang et al. (2007). "Effects of MDI spray angle on aerosol penetration efficiency through an oral airway cast." Journal of Aerosol Science 38(8): 853-864.

Farkas, A., I. Balashazy, et al. (2006). "Characterization of regional and local deposition of inhaled aerosol drugs in the respiratory system by computational fluid and particle dynamics methods." Journal of Aerosol Medicine-Deposition Clearance and Effects in the Lung 19(3): 329-343.

Fink, J. B. (2000). "Metered-dose inhalers, dry powder inhalers, and transitions." Respiratory Care 45(6): 623-35.

Fink, J.B., W. Wilkes et al. (1998). "A new method to characterize the effects of spacers and holding chambers on poor hand-breath coordination during MDI use?" Respiratory Care 43(10): 873.

Finlay, W. H. (2001). "The Mechanics of Inhaled Pharmaceutical Aerosols: An Introduction." Academic Press, London.

Finlay, W.H. and P. Zuberbuhler (1999). "In vitro comparison of salbutamol hydrofluoroalkane (Airomir) metered dose inhaler aerosols inhaled during pediatric tidal breathing from five valved holding chambers." Journal of Aerosol MedicineDeposition Clearance and effects in the Lung 12(4): 285-291.

Fischer, M., M. Raffel et al. (1994). "Combined particle image velocimetry and visualization experiments on instabilities in a flat plate boundary layer with zero pressure gradient." Optical Engineering 33(4): 1343-1348. 
Fletcher, G.E. (1975). "Factors affecting the atomization of saturated liquids." $\mathrm{PhD}$ thesis. Loughborough University of Technology. Loughborough, UK.

Gosman, A. D. and E. Ioannides (1981). "Aspects of Computer Simulation of Liquid Fuelled Combustors." AIAA Paper No. 81-0323.

Graham, S. J., R. C. Lawrence, et al. (1995). "Particle-Size Distribution of Single and Multiple Sprays of Salbutamol Metered-Dose Inhalers (Mdis)." Pharmaceutical Research 12(9): 1380-1384.

Han, R. J., G. Papadopoulos, et al. (2002). "Flow field measurement inside the mouthpiece of the Spiros inhaler using particle image velocimetry." Aerosol Science and Technology 36(3): 329-341.

Health Canada (2006). "It's your health: Asthma." Retrieved November 22, 2007, from http://www.hc-sc.gc.ca/iyh-vsv/diseases-maladies/asthm_e.html.

Heenan, A. F., W. H. Finlay, et al. (2004). "An investigation of the relationship between the flow field and regional deposition in realistic extra-thoracic airways." Journal of Aerosol Science 35(8): 1013-1023.

Heenan, A. F., E. Matida, et al. (2003). "Experimental measurements and computational modeling of the flow field in an idealized human oropharynx." Experiments in Fluids 35(1): 70-84.

Hiroyasu, H. and T. Kadota (1974). "Fuel droplet size distribution in diesel combustion chamber." SAE paper 740715 .

Hjemfelt, A.T., L.F. Mockros (1966). "Motion of discrete particles in a turbulent fluid." Applied Scientific Research 16:149.

Isaacs, K. K., R. B. Schlesinger, et al. (2006). "Three-dimensional computational fluid dynamics simulations of particle deposition in the tracheobronchial tree." Journal of Aerosol Medicine-Deposition Clearance and Effects in the Lung 19(3): 344-352.

Jin, H. H., J. R. Fan, et al. (2007). "Large eddy simulation of inhaled particle deposition within the human upper respiratory tract." Journal of Aerosol Science 38(3): 257-268.

Johnstone, A., M. Uddin, et al. (2004). "The flow inside an idealised form of the human extra-thoracic airway." Experiments in Fluids 37(5): 673-689.

Kakade, P. P., H. K. Versteeg; et al. (2007). "Design optimization of a novel pMDI actuator for systemic drug delivery." Journal of Aerosol Medicine-Deposition Clearance and Effects in the Lung 20(4): 460-474. 
Kleinstreuer, C., H. W. Shi, et al. (2007). "Computational analyses of a pressurized metered dose inhaler and a new drug-aerosol targeting methodology." Journal of Aerosol Medicine-Deposition Clearance and Effects in the Lung 20(3): 294-309.

Launder, B. E. and D. B. Spalding (1972). "Lectures in Mathematical Models of Turbulence." Academic Press, London.

LaVision (2006a). DaVis 7.1 Software Manual for DaVis 7.1. Goettingen, LaVision $\mathrm{GmbH}$.

LaVision (2006b). DaVis FlowMaster Software Manual for DaVis 7.1. Goettingen, LaVision $\mathrm{GmbH}$.

LaVision (2006c). DaVis FlowMaster Hardware Manual for DaVis 7.1. Goettingen, LaVision $\mathrm{GmbH}$.

LaVision (2005). Geometry Package Manual for DaVis 7.0. Goettingen, LaVision $\mathrm{GmbH}$.

Melling, A. (1997). "Tracer particles and seeding for particle image velocimetry." Measurement Science \& Technology 8(12): 1406-1416.

Meyers, J.F. (1991). "Laser Velocimetry, Generation of Particles and Seeding." Von Karman Institute for Fluid Dynamics, Lecture Series 1991-08. NASA Langley Technical Report Center, Virginia, USA.

Newman, S. P. (2005). "Principles of metered-dose inhaler design." Respiratory Care 50(9): 1177-90.

Newman, S.P. and M.T. Newhouse (1996). "Effect of add-on devices for aerosol drug delivery: Deposition studies and clinical aspects." Journal of Aerosol MedicineDeposition Clearance and Effects in the Lung 9(1):55-70.

Newman, S.P. (1993). "Scintigraphic assessment of therapeutic aerosols." Critical Reviews in Therapeutic Drug Carrier Systems 10(1): 65:109.

Newman, S.P., W.B. Weisz et al. (1991). "Improvement of drug delivery with a breath actuated pressurized aerosol for patients with poor inhaler technique." Thorax 46(10): 712-716.

Nogueira, J., A. Lecuona, et al. (1997). "Data validation, false vectors correction and derived magnitudes calculation on PIV data." Measurement Science \& Technology 8(12): 1493-1501. 
Prasad, A.K., R.J. Adrian et al. (1992). "Effect of resolution on the speed and accuracy of particle image velocimetry interrogation." Experiments in Fluids 13(2-3): 105-116.

Raffel, M., C.E. Willert et al. (1998). "Particle Image Velocimetry: A Practical Guide." Springer-Verlag, Berlin Heidelberg, Germany.

Rahmatalla, M. F., P. C. Zuberbuhler, et al. (2002). "In vitro effect of a holding chamber on the mouth-throat deposition of QVAR((R)) (Hydrofluoroalkane-beclomethasone dipropionate)." Journal of Aerosol Medicine-Deposition Clearance and Effects in the Lung 15(4): 379-385.

Ramasamy, M., and J.G. Leishman. "Benchmarking Particle Image Velocimetry with Laser Doppler Velocimetry for Rotor Wake Measurements." AIAA Journal 45(11): 2622:2633..

Stapleton, K.W., E. Guentsch, et al. (2000). "On the suitability of k-epsilon turbulence modeling for aerosol deposition in the mouth and throat: A comparison with experiment." Journal of Aerosol Science 21(6): 739-749.

Takano, H., N. Nishida, et al. (2006). "Inhaled particle deposition in unsteady-state respiratory flow at a numerically constructed model of the human larynx." Journal of Aerosol Medicine-Deposition Clearance and Effects in the Lung 19(3): 314-328.

Tarara, T. E., M. S. Hartman, et al. (2004). "Characterization of suspension-based metered dose inhaler formulations composed of spray-dried budesonide microcrystals dispersed in HFA-134a." Pharmaceutical Research 21(9): 1607-1614.

Vidgren, M.T., T.P. Paronen et al. (1987). "Effect of extension devices on the drug deposition from inhalation aerosols." International Journal of Pharmaceutics 39(12):107-112.

Westerweel, J. (1994). "Efficient Detection of Spurious Vectors in Particle Image Velocimetry Data." Experiments in Fluids 16(3-4): 236-247.

Wigley, G., H.K. Versteeg et al. (2002). "Near-orifice PDA measurements and atomisation mechanism of a pharmaceutical pressurised metered dose inhaler." Proceedings of the $18^{\text {th }}$ Annual Conference of Liquid Atomization \& Spray Systems $165-170$.

Willert, C., M. Raffel et al. (1996). "Recent applications of particle image velocimetry in aerodynamic research." Flow Measurement and Instrumentation 7(3-4): 247-256.

Wu, K. J., D. A. Santavicca, et al. (1984). "LDV Measurements of Drop Velocity in Diesel-Type Sprays.” AIAA Journal 22(9): 1263-1270. 